University of New Hampshire

University of New Hampshire Scholars' Repository

Winter 1996

\title{
Global terrestrial biogeochemistry: Perturbations, interactions, and time scales
}

Bobby H. Braswell

University of New Hampshire, Durham

Follow this and additional works at: https://scholars.unh.edu/dissertation

\section{Recommended Citation}

Braswell, Bobby H., "Global terrestrial biogeochemistry: Perturbations, interactions, and time scales" (1996). Doctoral Dissertations. 1927.

https://scholars.unh.edu/dissertation/1927

This Dissertation is brought to you for free and open access by the Student Scholarship at University of New Hampshire Scholars' Repository. It has been accepted for inclusion in Doctoral Dissertations by an authorized administrator of University of New Hampshire Scholars' Repository. For more information, please contact Scholarly.Communication@unh.edu. 


\section{INFORMATION TO USERS}

This manuscript has been reproduced from the microfilm master. UMI films the text directly from the original or copy submitted. Thus, some thesis and dissertation copies are in typewriter face, while others may be from any type of computer printer.

The quality of this reproduction is dependent upon the quality of the copy submitted. Broken or indistinct print, colored or poor quality illustrations and photographs, print bleedthrough, substandard margins, and improper alignment can adversely affect reproduction.

In the unlikely event that the author did not send UMI a complete manuscript and there are missing pages, these will be noted. Also, if unauthorized copyright material had to be removed, a note will indicate the deletion.

Oversize materials (e.g., maps, drawings, charts) are reproduced by sectioning the original, beginning at the upper left-hand corner and continuing from left to right in equal sections with small overlaps. Each original is also photographed in one exposure and is included in reduced form at the back of the book.

Photographs included in the original manuscript have been reproduced xerographically in this copy. Higher quality 6" 6 9" black and white photographic prints are available for any photographs or illustrations appearing in this copy for an additional charge. Contact UMI directly to order.

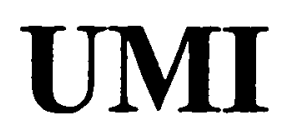

A Bell \& Howell Information Company 300 North Zeeb Road, Ann Arbor MI 48106-1346 USA

$313 / 761-4700 \quad 800 / 521-0600$ 
GLOBAL TERRESTRIAL BIOGEOCHEMISTRY: PERTURBATIONS, INTERACTIONS, AND TIME SCALES

BY

BOBBY H. BRASWELL. JR.

B.S., University of Alabama. 1987

M.S., University of New Hampshire, 1990

DISSERTATION

Submitted to the University of New Hampshire

in Partial Fulfillment of

the Requirements for the Degree of

Doctor of Philosophy

in

Earth Sciences

December, 1996 
UMI Number: 9717851

UMI Microform 9717851

Copyright 1997, by UMI Company. All rights reserved.

This microform edition is protected against unauthorized copying under Title 17, United States Code.

\section{UMI \\ 300 North Zeeb Road \\ Ann Arbor, MI 48103}


This dissertation has been examined and approved.

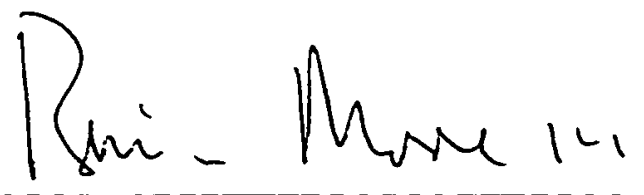

Dissertation director, Dr. Berrien Moore III

Director, Institute for the Study of Earth, Oceans and Space

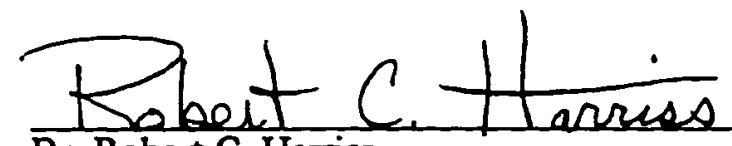

Dr. Robert C. Harriss

Professor of Earth Sciences

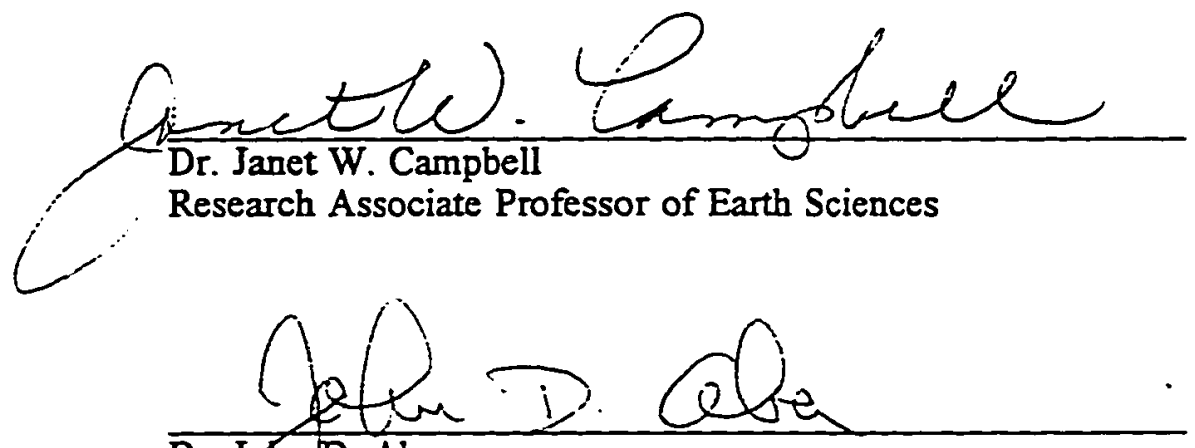

Dr. Jøhn D. Aber

Professor of Natural Resources

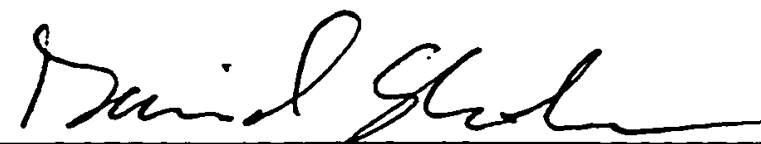

Dr. David L. Skole

Research Assistant Professor
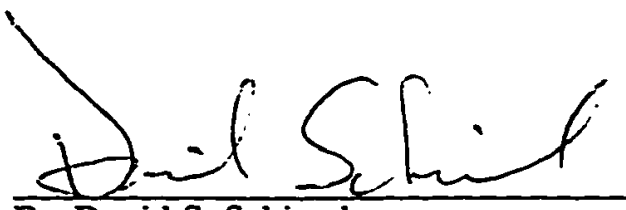

Dr. David S. Schimel

Senior Scientist, National Center for Atmospheric Research

$$
\frac{12-4-96}{\text { Date }}
$$




\section{DEDICATION}

This dissertation is dedicated to the memory of

James D. and Ruth B. Warren.

iii 


\section{ACKNOWLEDGMENTS}

Many thanks to

Robert Harriss, Berrien Moore, David Schimel, and Beth Holland, for advice, inspiration, and encouragement;

Alan Townsend, Jeff Privette, Becky McKeown, Elizabeth Sulzman, Nan Rosenbloom, Hank Fisher, James Sulzman, Greg Asner, Jay Famiglietti, Tim Kittel, Tom Painter, Steve Frolking, Charlie Vorosmarty, and Ernst Linder for collegial friendship, helpful discussions.

programming help, and/or data;

Susan Chavez, Gaylynn Potemkin, and Karen Bushold for many bureaucratic and logistic rescues;

Mom, Dad, and the rest of my family for doing what they did;

Kate, Anna, their parents, and Teal: the finest family a dirtbag could crash on;

Larisa Yaskell, Graham Smith, 4 McDonough Street, Jennifer Belkus, Jessica Belkus, Peter Duchesne, Angela Plaia, the Reverend Garen Heller, The HÜMPMÜSCLE Rolling Circus, God, and anyone else who inadvertently or intentionally helped me do this;

C. Nevison, E. Matthews, P. Vitousek, J. Neff, R. Howarth, S. Solomon, E. Sulzman, P. Tans, M. Hartman, B. McKeown, S. Jacquemoud, and A. Kuusk, for contributions to the previously published work in this dissertation;

Financial support was provided by (1) A fellowship appointment to the U.S. Department of Energy, Graduate Fellowships for Global Change (administered by the Oak Ridge Institute for Science and Education); (2) The University Corporation for Atmospheric Research, Climate System Modeling Program; and (3) The National Aeronautics and Space Administration, EOS Interdisciplinary Science Program. 
TABLE OF CONTENTS

ACKNOWLEDGMENTS _____ iv

LIST OF TABLES __ vii

LIST OF FIGURES __ viii

ABSTRACT

$\begin{array}{ll}\text { CHAPTER } & \text { PAGE }\end{array}$

1. INTRODUCTION AND OVERVIEW ___ 1

2. ATMOSPHERIC CARBON DIOXIDE AND THE IMPORTANCE OF THE TERRESTRIAL BIOSPHERE ___ 6

2.1 Introduction $\quad 6$

2.2 Adding up the Global Carbon Budget __ 11

2.3 The Lifetime of Excess Atmospheric $\mathrm{CO}_{2}$ (Moore and Braswell 1994) ___ 30

2.4 Complement: Timescales of Atmospheric $\mathrm{CO}_{2}$ and Radiative Forcing Indices

2.5 Stabilization Scenarios and Model Sensitivity

2.6 Summary

3. ATMOSPHERIC NITROGEN DEPOSITION AND THE GLOBAL CARBON CYCLE

3.1 Introduction

3.2 Spatial and Temporal Patterns in Terrestrial Carbon Storage due to Deposition of Fossil Fuel Nitrogen (Townsend et al. 1996)

3.3 The Spatial Distribution of Atmospheric Nitrogen Deposition and its Impact on Carbon Uptake by Terrestrial Ecosystems (Holland et al. In press)

3.4 Factors Limiting Ecosystem Response to N Additions

3.5 Summary 138

4. EQUILIBRIUM AND TRANSIENT SYSTEM-LEVEL RESPONSE OF TERRESTRIAL BIOGEOCHEMICAL DYNAMICS: MODELS AND OBSERVATIONS

4.1 Introduction

4.2 Climate and Nitrogen Controls on the Geography and Time Scales of 
Terrestrial Biogeochemical Cycling (Schimel et al. In press)

4.3 Characteristic Responses of Global Terrestrial Ecosystems to Temperature Inferred from Satellite and Surface Data

4.4 Summary

5. INTEGRATING REMOTE SENSING AND PHYSICAL MODELING TO ESTIMATE THE STATUS OF TERRESTRIAL VEGETATION

5.1 Introduction

5.2 Empirical Approaches: Vegetation Indices

5.3 Extracting Ecological and Biophysical Parameters from AVHRR Optical Measurements: An Integrated Algorithm Based on Inverse Modeling (Braswell et al. In press)

5.4 Toward Remote Sensing Data Assimilation for Terrestrial Modeling 246

5.5 Summary 250

6. CONCLUSIONS 252

6.1 Review of Chapter Contributions 252

6.2 Future Work 253

APPENDIX A. THE SAIL MODEL EQUATIONS 256

APPENDIX B. DETAILS OF THE INTEGRATED INVERSION ALGORITHM 262 


\section{LIST OF TABLES}

\section{TABLE}

PAGE

2.1 The average annual budget of anthropogenic $\mathrm{CO}_{2}$ for 1980-1989 28

2.2 The differential equations in the Emanuel et al. (1984) terrestrial model __ 42

2.3 Single Half-Life results for the ocean-atmosphere models _ 52

2.4 Single Half-Life results for the ocean-atmosphere-terrestrial models

2.5 Coefficient of correlation $(R)$ for the fit of $F_{r}$ to the calculated residual carbon flux 52

2.6 Sensitivity of $T_{\text {res }}$ for the ocean-atmosphere models _ 59

2.7 Goodness of fit for selected $T_{\text {res }}$ calculations __ 59

2.8 Sensitivity of $T_{\text {res }}$ for the E-BD ocean-atmosphere-terrestrial model __ 62

3.1 Biome-specific parameters used in the NDEP model __ 87

$3.2 \mathrm{NO}_{\mathrm{x}}$ sources in $\mathrm{Tg} \mathrm{N} \mathrm{yr} \mathrm{H}^{-1}$ for five 3-D chemical transport models 106

$3.3 \mathrm{NO}_{\mathrm{y}}$ deposition on the Earth's surface resulting from fossil fuel combustion 115

3.4 Total $\mathrm{NO}_{\mathrm{y}}$ deposition on the Earth's surface considering all $\mathrm{NO}_{\mathrm{x}}$ sources 117

3.5 Comparison of terrestrial net $\mathrm{C}$ flux from NDEP and from inverse $\left[\mathrm{CO}_{2}\right]$ modeling _ 127

3.6 Results of NDEP simulations using high and low MOGUNTIA deposition ___ 135

5.1 The SAIL-2 model parameters, and their bounds or default values ___ 222

5.2 Comparison of broad-band albedo estimates from the SAIL-2 inversions and SiB _ 242 


\section{LIST OF FIGURES}

1.1 Schematic depicting anthropogenic perturbations to the global climate system 2

2.1 Historical atmospheric $\mathrm{CO}_{2}$ concentrations _ 7

2.2 The fossil fuel emissions record 8

2.3 The global carbon cycle ___ 12

2.4 Box-diffusion model 35

2.5 Outcrop-diffusion model __ 35

2.6 Twelve-box model

2.7 Ocean-atmosphere model response: fossil fuel forcing 38

2.8 Ocean-atmosphere model response: fossil fuel plus biotic forcing __ 39

2.9 Globally averaged carbon cycle model 43

2.10 Deconvolved flux determined by the ocean-terrestrial-atmosphere model 48

2.11 Deconvolved flux per NPP, as a function of atmospheric $\left[\mathrm{CO}_{2}\right] \longrightarrow 49$

2.12 Ocean-atmosphere models response to a cessation of all emissions _ 53

$2.13 \mathrm{E}-12 \mathrm{~B}$ model response to the emissions cutoff scenario

2.14 Response of the ocean-atmosphere models to an instantaneous pulse of $\mathrm{CO}_{2} \_58$

2.15 Box-diffusion model response to a pulse, and two fitted curves __ 61

$2.16 \mathrm{IPCC}$ scenarios for prescribed stabilization of atmospheric $\left[\mathrm{CO}_{2}\right] \longrightarrow 70$

2.17 Deconvolved fossil fuel flux for IPCC stabilization 71

2.18 Deconvolved fossil fuel flux for IPCC stabilization, with reduced fertilization _

2.19 Vegetation carbon increase for IPCC stabilization 74

2.20 The effect of the inclusion of $\mathrm{CO}_{2}$ fertilization in the IPCC stabilization 75

2.21 The decay of a $10 \mathrm{Pg} \mathrm{C}$ pulse of $\mathrm{CO}_{2}$ added to the model atmosphere

3.1 Carbon and nitrogen pools and fluxes in the NDEP model

3.2 Stored carbon resulting from fossil $\mathrm{N}$ deposition over time 93

3.3 Latitudinal distribution of the net carbon sink due to fossil $\overline{\mathrm{N} \text { deposition ___ }} 94$

3.4 Spatial distribution of the 1990 carbon sink and the deposition pattern -97

3.5 Sensitivity of the estimated carbon sink to model parameters 98

$3.6 \mathrm{~A}$ comparison of simulated and measured wet $\mathrm{NO}_{3}{ }^{\circ}$ deposition 110

3.7 The latitudinal distribution of total $\mathrm{NO}_{\mathrm{y}}$ distribution 112

3.8 Global distribution of total $\mathrm{NO}_{\mathrm{y}}$ deposition simulated by IMAGES 113

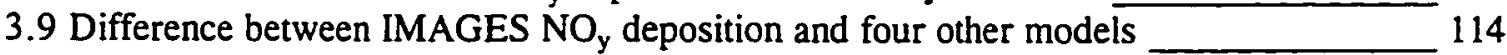

3.10 Globally integrated carbon storage from total $\mathrm{NO}_{\mathrm{y}}$ deposition calculated by NDEP -118

3.11 Latitudinal distribution of total $\mathrm{NH}_{\mathrm{x}}$ deposition and modeled carbon storage

3.12 Modeled global distribution of carbon storage due to $\mathrm{NO}_{\mathrm{y}}$ plus $\mathrm{NH}_{\mathrm{x}} \longrightarrow 122$

3.13 Latitudinal distribution of the carbon sink due to $\mathrm{NO}_{\mathrm{y}}$ and $\mathrm{NOy}+\mathrm{NH}_{\mathrm{x}} \longrightarrow 126$

3.14 Ozone concentrations observed and simulated by IMAGES 131

3.15 Schematic representing the parameterization of nitrogen saturation in NDEP __ 133

3.16 Time series of $\mathrm{N}$ and $\mathrm{C}$ fluxes for one forest grid cell 136

3.17 Time series of accumulated $\mathrm{N}$ and $\mathrm{C}$ for one forest grid cell 137

4.1 The Century terrestrial biogeochemical model _ـ 148 
4.2 The cyclic coupling of carbon, water flux, and nitrogen cycle processes 156

4.3 Mapped ET, NMIN, and NPP

4.4 Pairwise relationships between ET, NMIN, and NPP

4.5 Temperature anomalies from the Microwave Sounding Unit 162

4.6 Correlations for $\mathbf{5 2}$ grid cells between temperature and carbon fluxes 163

4.7 Modeled effect of the Pinatubo temperature anomaly on NEP 165

4.8 Results from a Century temperature pulse experiment 166

4.9 Century response to one-year and five-year temperature perturbations 168

4.10 Time series of global and hemispheric mean temperature, NDVI, and $\overline{\left.\mathrm{CO}_{2}\right]} 179$

4.11 Autocorrelation of temperature, NDVI, and $\left[\mathrm{CO}_{2}\right]$ time series 182

4.12 Cross-correlation of temperature, NDVI, and $\left[\mathrm{CO}_{2}\right]$ time series 183

4.13 Spatial distribution of correlation timescales of temperature and NDVI 185

4.14 Global and hemispheric means of standardized regression coefficients 189

5.1 Schematic depicting the configuration of sun and satellite sensor 199

5.2 Typical leaf and soil reflectance spectra, and the AVHRR bandwidths 201

5.3 Visible and NIR reflectance simulated by the SAIL model 214

5.4 SAIL model simulations showing parameter sensitivity 215

5.5 Phenological characterization of Africa, with study transect

5.6 AVHRR-convolved leaf reflectance and transmittance data

5.7 AVHRR-convolved soil reflectance data

5.8 Sun-sensor phase angle and reflectances for a typical site 234

5.9 A typical cell characterized by a PC analysis of multi-temporal NDVI data 235

5.10 Retrieved FAPAR along the transect 238

5.11 Retrieved $F A P A R$ along the transect, combined, as a function of time 239

5.12 A comparison of $F A P A R$ from the SAIL-2 inversion and from SiB 241

B. 1 Flow chart describing the inverse model algorithm 263

B.2 A discrete classification of Africa using the first three PCs of $\overline{\text { NDVI }}$

B.3 Semivariogram contours of $\overline{\mathrm{NDVI}}$ 265

B.4 Polar plots of the view zenith (Radians) and azimuth for aggregated data 


\begin{abstract}
GLOBAL TERRESTRIAL BIOGEOCHEMISTRY: PERTURBATIONS, INTERACTIONS, AND TIME SCALES
\end{abstract}

by

Bobby H. Braswell, Jr.

University of New Hampshire, December. 1996

Global biogeochemical processes are being perturbed by human activity, principally that which is associated with industrial activity and expansion of urban and agricultural complexes. Perturbations have manifested themselves at least since the beginning of the 19th Century, and include emissions of $\mathrm{CO}_{2}$ and other pollutants from fossil fuel combustion, agricultural emissions of reactive nitrogen, and direct disruption of ecosystem function through land conversion. These perturbations yield local impacts, but there are also global consequences that are the sum of local-scale influences.

Several approaches to understanding the global-scale implications of chemical perturbations to the Earth system are discussed. The lifetime of anthropogenic $\mathrm{CO}_{2}$ in the atmosphere is an important concept for understanding the current and future commitment to an altered atmospheric heat budget. The importance of the terrestrial biogeochemistry relative to the lifetime of excess $\mathrm{CO}_{2}$ is demonstrated using dynamic, aggregated models of the global carbon cycle. A central theme is the annual flux of carbon into the terrestrial biosphere. Several mechanisms for modification of the natural amount of terrestrial carbon uptake are discussed, focusing on the effects of pollutant deposition; we estimate the historical flux of 
carbon due to nitrogen deposition, and its sensitivity to assumptions about the details of ecosystem function and to the accuracy of predicted deposition patterns. Further, we introduce the hypothesis that internal biogeochemical regulation results in extreme interannual fluctuations of carbon exchange. This hypothesis is evaluated, and found to be consistent with global data sets of temperature, atmospheric $\mathrm{CO}_{2}$ concentrations, and land surface reflectance. Satellite remote sensing of vegetation amount and function is one of the most important sources of information about the perturbed terrestrial biosphere. Traditional techniques for using optical reflectance data are discussed, and an unconventional algorithm is introduced, based on the inversion of a plant canopy radiative transfer model. The observation of the land surface at multiple angles is critical in this method. Successful model inversions are performed on a transect in the Central African Republic. We extracted biophysical quantities, as well as implicit information about phenology. This technique will be most useful when proposed satellite instruments provide angular reflectance information. 


\section{CHAPTER I}

\section{INTRODUCTION AND OVERVIEW}

The Earth's coupled climate-biogeochemical system is dynamic, exhibiting large scale trends and fluctuations that can be seen in observational records of the recent, as well as distant, past. Currently, global changes are occurring that are due to the cumulative effects of human activity, and that are proceeding at a more rapid rate (with few exceptions) than prehistoric variability. Global-scale anthropogenic forcings are primarily associated with industrial metabolism (e.g., fossil fuel combustion) and the expansion of agriculture (including animal husbandry), driven by population growth and changing societal demands. Virtually all largescale human behavior carries with it multiple impacts on the Earth system (Fig. 1.1), but the scientific issues are complex, and political decisions that seek to slow or reverse the course of change are rare.

Despite the existing controversy, there are some reliable indicators of global change. For example, the concentrations of radiatively important trace gases (e.g., $\mathrm{CO}_{2}, \mathrm{CH}_{4}, \mathrm{~N}_{2} \mathrm{O}$ ) are steadily rising and causing serious concern about the heat budget of the atmosphere and the possible associated climatic effects (such as sea level rise). The magnitude, timing, and spatial distribution of any impacts arising from "global warming" can be estimated by models, but they are not easily verified. In addition to the issue of climate change, the same human activities (mentioned above) are directly responsible for the chemical and physical alteration of terrestrial ecosystems. Because the biosphere's critical tole in the Earth system, direct and indirect (climate related) changes can cause biologically-mediated feedbacks, both positive and 


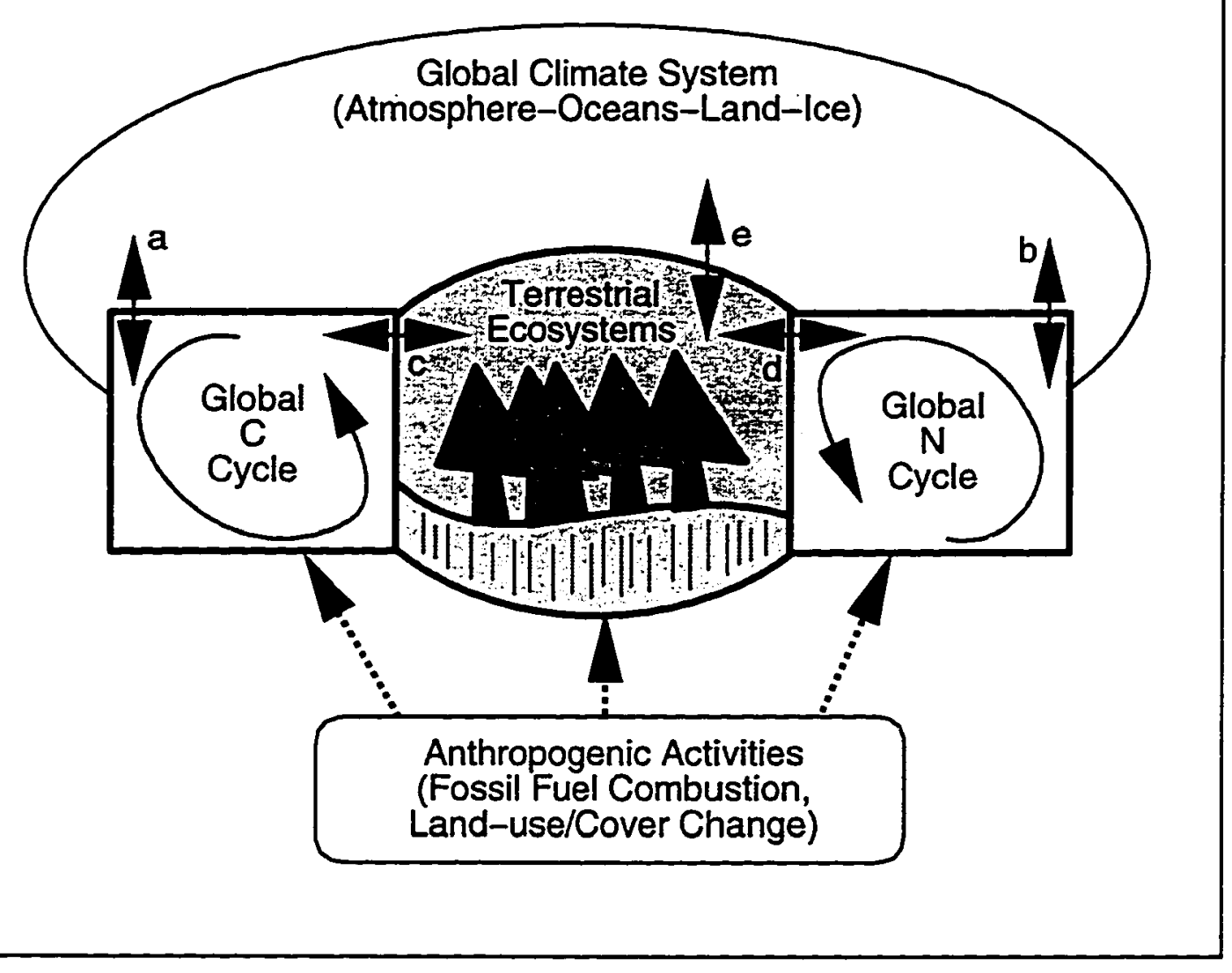

Figure 1.1 Schematic depicting anthropogenic perturbations to the global climate system via direct impacts on overlapping, coupled sub-systems: the carbon cycle, the nitrogen cycle, and terrestrial ecosystems. (a) Atmospheric $\mathrm{CO}_{2}$ concentrations; (b) Atmospheric concentrations of $\mathrm{N}_{2} \mathrm{O}$ and $\mathrm{O}_{3}$ (affected by $\mathrm{NO}_{x}$ chemistry); (c) and (d) Terrestrial uptake, cycling, and losses of $\mathrm{C}$ and $\mathrm{N}$; (e) Water, energy, and momentum exchange with the atmosphere. 
negative. Understanding the balance of these feedbacks is critical and, in part, the subject of this dissertation.

The following chapters $(2-5)$ represent four separate, but related, investigations into the central importance of terrestrial ecosystems in regulating the response of global biogeochemical cycles to human-induced perturbations. We focus particularly on the interactive cycles of carbon and nitrogen. A common theme in all four chapters is the use of system-dynamic or physical models in conjunction with global data sets to characterize the changing state of the terrestrial biosphere. Each chapter has a somewhat different focus; we begin with a global view of the carbon cycle, then proceed to more process-oriented analyses of the terrestrial biosphere, and finally develop methods for estimating terrestrial processes using satellite observations. We present a brief overview in the remainder of this chapter.

Chapter 2: Atmospheric carbon dioxide and the importance of the terrestrial biosphere. We review the issues surrounding the budget of anthropogenically produced $\mathrm{CO}_{2}$ and the uncertainty associated with the magnitude and distribution of net terrestrial carbon exchange. We use a highly aggregated (but relatively sophisticated) model of terrestrial and oceanic carbon cycling to investigate the historical evolution of the terrestrial term, and the possible significance of direct $\mathrm{CO}_{2}$ fertilization of the biosphere. We further step into a somewhat controversial arena that is related to enhanced greenhouse warming: characterizing the atmospheric lifetime of anthropogenic $\mathrm{CO}_{2}$. We show that conventional methods for estimating the duration of commitment to $\mathrm{CO}_{2}$ forcing are not robust due to the asymptotic and nonlinear behavior of carbon cycle dynamics. These complications arise primarily because of ocean chemistry and the complexity of terrestrial ecosystems. Thus, we had difficulty arriving at an alternative index. Though our original intent was to provide an unequivocal measure for use in calculating Greenhouse Warming Potentials, our index (the Single Half-Life) was used to 
characterize the importance of terrestrial feedbacks on the short-term (interannual) dynamics of atmospheric $\mathrm{CO}_{2}$. This chapter is based on work by Moore and Braswell (1994).

Chapter 3: Atmospheric nitrogen deposition and the global carbon cycle. One of the issues raised in Chapter 2 was the well-known possibility of terrestrial fertilization by deposition of anthropogenically fixed nitrogen. This reactive nitrogen can end up augmenting the mineral pool available to plants and microbes (i.e., $\mathrm{NO}_{3}{ }^{-}$and $\mathrm{NH}_{4}{ }^{+}$). We present an analysis of the terrestrial $\mathrm{C}$ sink potential associated with $\mathrm{N}$ deposition using existing global data plus models of atmospheric chemistry and transport, within a simple dynamic modeling framework that we developed. Our goal was to present an estimate of $\mathrm{N}$ fertilization that accounted for the overlying spatial patterns of deposition and the distribution of ecosystems which have varying capacities to utilize the excess $\mathrm{N}$. In addition to this estimate, our investigations highlighted uncertainties associated with mechanistic-level terrestrial and atmospheric controls. Our framework for analysis is expandable to include more detailed parameterization of terrestrial response to chronic deposition of $\mathrm{N}$ and other pollutants, as well as more refined (and internally consistent) estimates of deposition. This chapter is based on work presented by Townsend et al. (1996) and Holland et al. (In press).

\section{Chapter 4: Equilibrium and transient system-level response of terrestrial} biogeochemical dynamics: models and observations. Variability and trends in climate was introduced in Chapter 2 as another possible mechanism for modifying net atmosphere-biosphere carbon exchange. We look in detail at the complexity of the terrestrial climate- $\mathrm{CO}_{2}$ relationship, including the importance of internal biogeochemical cycling of nutrients (primarily nitrogen) and water. We develop a hypothesis about the relative importance of biotic versus abiotic controls over equilibrium carbon exchange by terrestrial ecosystems based on analysis of a global process model simulation. A corollary to this hypothesis is related to time scales of 
response of terrestrial ecosystems (and net $\mathrm{CO}_{2}$ exchange) to perturbations. We evaluate the model-based results using the 12-15 year record of global remote sensing data (temperature and vegetation index) and measurements of atmospheric $\mathrm{CO}_{2}$ concentrations. Far from providing a straightforward answer, we show with some confidence that issues related to interannual armosphere-terrestrial carbon exchange are complex, exhibiting individualistic responses at local-to-regional scales. The first part of this chapter is based on work by Schimel et al. (In press a), and the second part (related to the analysis of global data) is the subject of a future article (Braswell et al. In preparation).

Chapter 5: Integrating remote sensing and physical modeling to estimate the status of terrestrial vegetation. Problems interpreting the remotely-sensed vegetation index led us in Chapter 4 to attempt to use more indirect statistical methods to investigate interannual changes in terrestrial vegetation. In this chapter we take a close look at the information content of optical remote sensing data, focusing on the dependence of reflectance on the relative positions of sun and sensor (bidirectional effects). We present a method for obtaining biophysical information about ecosystems from satellite data that uses physical models of canopy light environment in an "inverse-mode". Factors that obscure the results of other methods actually provide useful information in this approach. This chapter is based on work by Braswell et al. (1996). 


\section{CHAPTER 2}

\section{ATMOSPHERIC CARBON DIOXIDE AND THE IMPORTANCE OF THE TERRESTRIAL BIOSPHERE}

\subsection{Introduction}

The pool of carbon in the atmosphere (in the form of $\mathrm{CO}_{2}$ ) has increased from about 590 to almost $755 \mathrm{Pg}$ ( $1 \mathrm{Pg}=1 \times 10^{15} \mathrm{~g}=1$ billion metric tons) during the last $200-250$ years. Presently, the annual rate of increase is about $2 \mathrm{ppm}$ (parts per million by volume) per year, an amount equivalent to roughly $0.6 \%$ per year. We have a direct record of this increase since 1958 (Keeling 1986), and a number of indirect records from ice cores (e.g., Neftel et al. 1985 , Raynaud and Barnola 1985, Friedli et al. 1986, Siegenthaler and Oeschger 1987) and sediments (e.g., White et al. 1994) of the increase over the past two centuries. These records show that the concentration of $\mathrm{CO}_{2}\left(\left[\mathrm{CO}_{2}\right]\right)$ has increased by more than $25 \%$ since the mid-1700s (Fig. 2.1). Moreover, from the same data we know that the concentration of carbon dioxide was relatively constant from the beginning of the present interglacial period (ca. 10,000 BP) to the onset of increases in the 18th century (e.g., Siegenthaler 1989). Thus, it is often assumed that carbon fluxes between the major reservoirs (atmosphere, ocean, and terrestrial biosphere) were nearly balanced prior to that time, and that the carbon cycle as a whole was in approximate steady state. This assumption is central to research that focuses on recent changes in the carbon cycle associated with increasing atmospheric $\left[\mathrm{CO}_{2}\right]$. There is strong evidence that these changes are the result of human perturbations to the natural carbon cycle.

The primary human activities contributing to changes in the natural cycling of carbon 


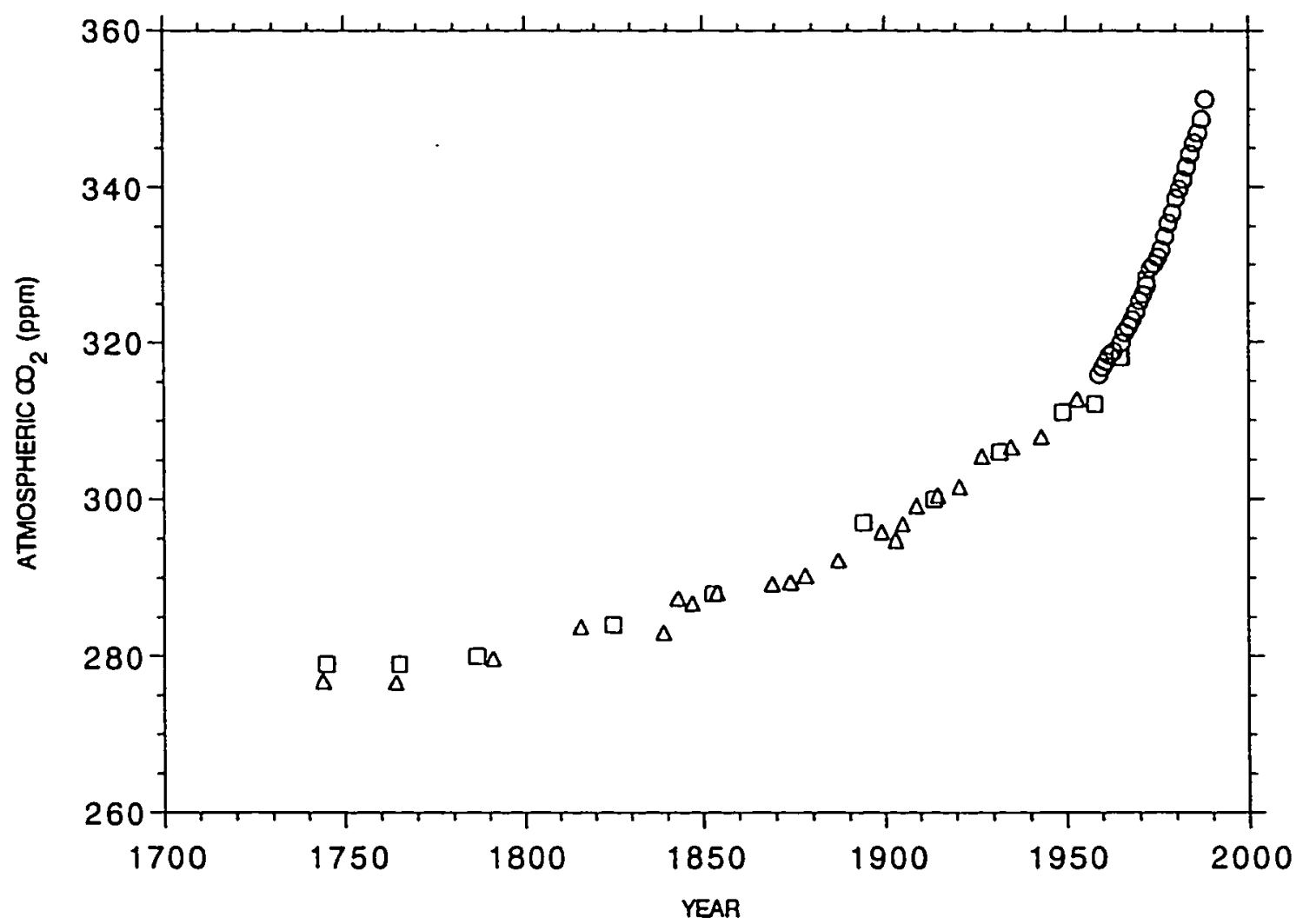

Figure 2.1 Historical atmospheric $\mathrm{CO}_{2}$ concentrations. The open squares and triangles represent data derived from ice core measurements, Siple Station, Antarctica. The open circles are the annual averaged atmospheric measurements from Mauna Loa Observatory (Keeling 1986). 


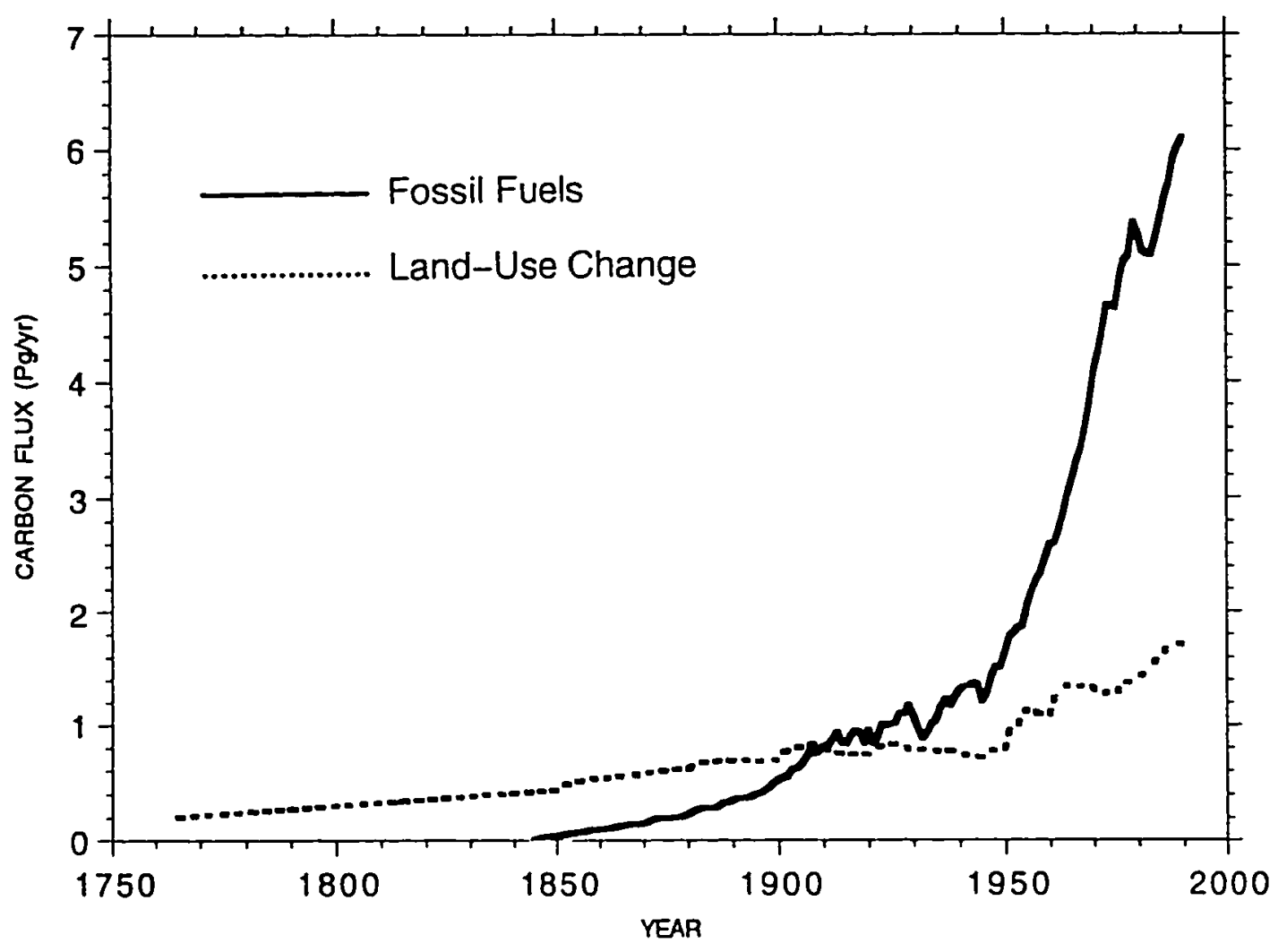

Figure 2.2 The fossil fuel emissions record $\left(F_{F E}\right)$ (Rotty and Marland 1986, Marland et al. 1994 ) is accurate to within about $10 \%$, but the estimate of biotic flux due to land-use change $\left(F_{B}\right)$ (Houghton 1983b,1985) is less certain, especially for the less recent numbers, because of a lack of a detailed historical accounting of global land-use patterns. 
are fossil fuel combustion and modifications of global vegetation through land-use (e.g., biomass burning, conversion to agriculture, abandonment of agriculture). For the period 19801989, an average of 5.4 $\mathrm{Pg} \mathrm{C}$ per year as $\mathrm{CO}_{2}$ was released to the atmosphere from the burning of fossil fuels (Marland et al. 1994), and it is estimated that an average of about $0.6-2.6 \mathrm{Pg} \mathrm{C}$ per year was emitted due to deforestation and land-use change during the same interval (e.g., Houghton and Skole 1990, Watson et al. 1990, Bolin and Fung 1992, Skole and Tucker 1993, and Houghton 1993b). Figure 2.2 shows an estimate of these two fluxes of anthropogenic $\mathrm{CO}_{2}$ from the mid-18th century to the present. In addition to fossil emissions and net land-use emissions (including uptake due to recovering/regrowing ecosystems), the set of carbon flux perturbations includes enhanced atmosphere-ocean exchange due to increased atmospheric $\left[\mathrm{CO}_{2}\right]$ and altered net terrestrial exchange due to chemical modification by atmospheric pollutants. All the human-derived (or altered) carbon fluxes listed above have in common the fact that, for each, the atmosphere is either donor or recipient; thus the atmosphere is literally central to understanding the carbon cycle. Finally, there are a number of possible second-order anthropogenic effects, including ocean and terrestrial feedbacks arising from anthropogenicallyderived changes in climate.

With respect to the modern, human-influenced carbon cycle, there are a number of classes of issues that should be addressed. These issues typically are defined by one or more of the questions listed below.

How have the major pools and fluxes of $\mathrm{CO}_{2}$ changed over the last approximately 200 years? This problem typically requires the use of simple, aggregated dynamic models and longterm, but very coarse spatial resolution data sets (e.g., atmospheric data, country-by-country land use clearing rates, and sediment/ice core measurements). A globally averaged, but somewhat temporally detailed picture of the carbon cycle allows investigation of the dominant 
$\mathrm{CO}_{2}$ exchange mechanisms, how they might change, and how they affect the long-term commitment to anthropogenic radiative forcing (Section 2.3).

What are the spatial patterns of global carbon sources and sinks, and how are they affected by climate and human intervention? Ecological, physical, and chemical process modeling is one approach to this issue. Analysis of atmospheric data (including atmospheric transport modeling) is another method that can also be used in concert with process models. "Spatial patterns" may refer to latitudinal distributions, geographically referenced (grid cell) products, or simply that one region has been distinguished from another. We consider aspects of this question in Chapters 3 and 4.

What are the principal terrestrial and oceanic mechanisms that control the global carbon cycle's response to the anthropogenic perturbations? This question typically requires integrated theoretical and experimental (field) studies. In some cases, the results of such studies will feed into parameterizations of carbon cycle models or process models (terrestrial or oceanic) that include carbon exchange with atmosphere. In addition, attributes, as well as objectives, of observational experiments can be guided by model-based analysis. We consider aspects of this question in Chapter 4 .

What are the present day exchanges of $\mathrm{CO}_{2}$ between the atmosphere and the other parts of the carbon cycle? The "present day," in this context, often indicates an averaging of excess $\mathrm{CO}_{2}$ fluxes over some period (e.g., 1980-1990), but can refer to a single year. Results from all the above types of analyses can be included in order to specify terms in the global budget of carbon (Section 2.2).

This chapter focuses on developing a broad but quantitative understanding of the contemporary carbon cycle as modified by human activity. In Section 2.2 , the issue of closing the budget of anthropogenic $\mathrm{CO}_{2}$ is discussed, including possible mechanisms for enhanced 
terrestrial uptake, and it is pointed out that there is more to balancing the carbon cycle than having a residual mean of zero. In Section 2.3 (also Moore and Braswell 1994), we discuss the importance of possible responses of the terrestrial biosphere in determining future levels of atmospheric $\mathrm{CO}_{2}$ by taking a system-dynamics approach and looking at characteristic response times. This section is the principal contribution of the chapter, and utilizes global-aggregated models (mentioned above). Section 2.4 is a follow-up to Moore and Braswell (1994); we respond to a commentary on our paper (Gaffin et al. 1995), and discuss implications and implementation of Greenhouse Warming Potential (GWP) indices. In the final section, a particular policy-related question will be discussed: according to our global carbon cycle model, what future changes in anthropogenic activity would be required to stabilize atmospheric $\mathrm{CO}_{2}$ at some level? This portion of our work formed a contribution to a recent Intergovernmental Panel on Climate Change (IPCC) report. We will also look at the sensitivity of those stabilization results to assumptions about the terrestrial biosphere.

\subsection{Adding Up The Global Carbon Budget}

The vector sum of all fluxes $\left(F_{i}\right)$ into and out of the atmosphere must equal the rate of increase in the concentration of atmospheric carbon dioxide $\left(d\left[\mathrm{CO}_{2}\right] / d t=\Sigma_{i} F_{i}\right)$. This equation is commonly referred to as the global carbon budget, though the number of terms is variable, and the uncertainty associated with many of the terms is large. In this section we will discuss the issues surrounding an ongoing attempt to balance the global budget of $\mathrm{CO}_{2}$. Such work generally treats each term separately, as each is associated with a separate set of processes. The principal carbon cycle fluxes (Fig. 2.3) are associated with terrestrial photosynthesis (primary production), plant and soil respiration, and $\mathrm{CO}_{2}$ exchange at the air-sea interface, all of which are modified by climate and human interference. Fluxes that result directly from human activity 
THE GLOBAL CARBON CYCLE

Approximate Reservoirs and Annual Fluxes 1980-1989 (Pg C)

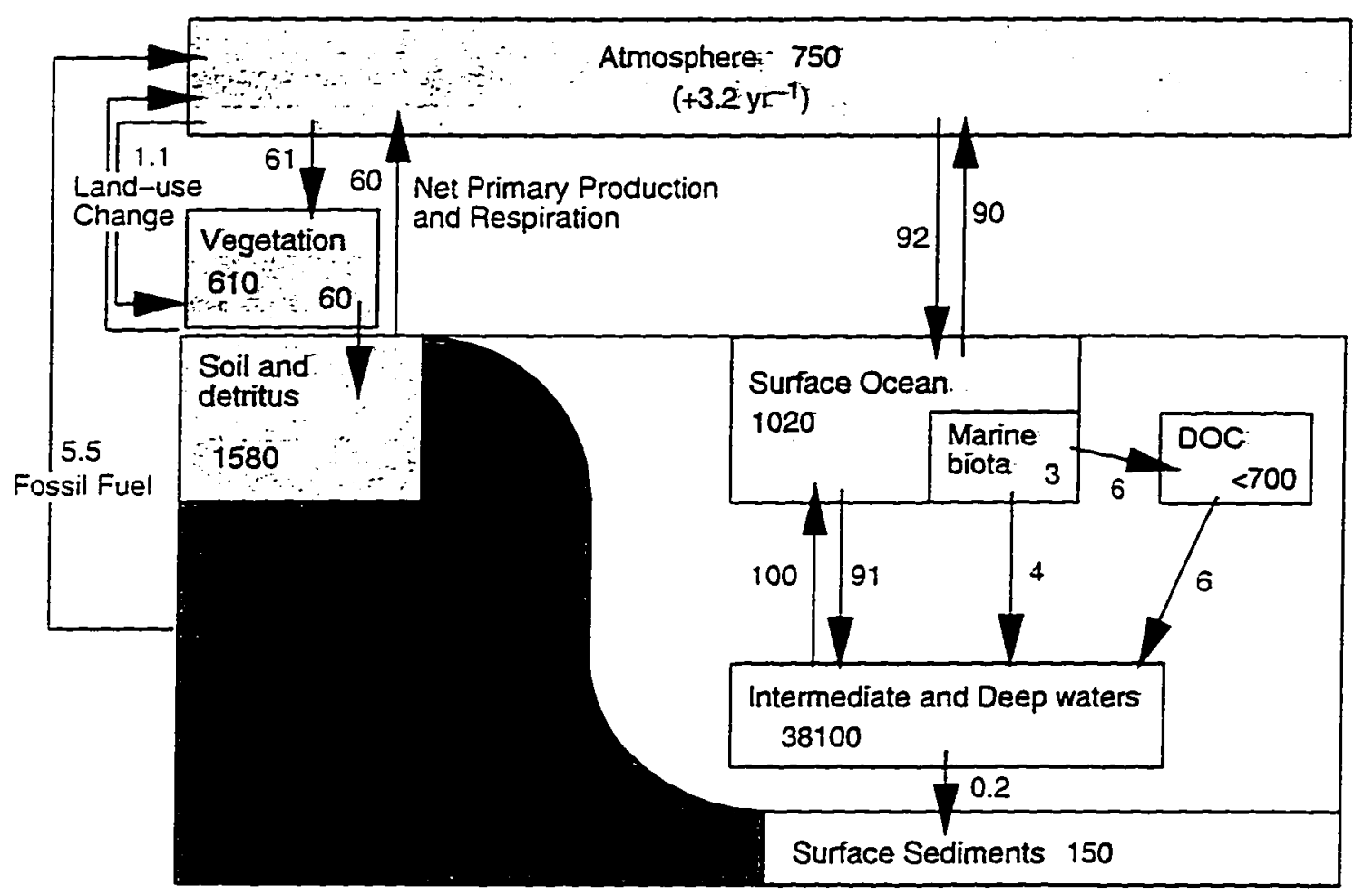

Figure 2.3 The global carbon cycle (IPCC; Schimel et al. 1995). The values within compartments are in units of $\mathrm{PgC}$, and the fluxes (arrows) are in units of $\mathrm{Pg} \mathrm{C} \mathrm{yr}^{-1}$. 
are associated with combustion of fossil fuels and land-use/cover change. The importance of balancing the carbon budget lies in the fact that an ability to quantify the world's carbon fluxes using disparate techniques would demonstrate a deep understanding at the mechanistic level. Given a clear mechanistic picture, models can be constructed and predictions made about future levels of $\mathrm{CO}_{2}$ in the atmosphere.

The global carbon budget is a well-defined concept, but an imprecise idiom has been used in the literature when discussing one aspect of uncertainty in the balance of fluxes of carbon: all available flux estimates are treated as known, and the residual difference between their sum and the estimated rate of change in $\left[\mathrm{CO}_{2}\right]$ is an amount of carbon that is said to be "missing". In the absence of a perfectly balanced budget, there is always a residual, and it is a missing source or a missing sink. This language is a well-understood shorthand and a convenient way to introduce a discussion; however. it tends to mask the considerable complexity associated with large uncertainties in many of the flux estimates. In addition, the balance of the carbon budget likely changes from year to year, and thus, comparisons of flux terms that are averages over one or many years must be made with care.

In this section. we will review the recent history of the community effort to probe the carbon budget. The discussion will be centered around a relatively large budget imbalance which has caused a great deal of speculation on the "location of a missing sink" for atmospheric $\mathrm{CO}_{2}$, and its subsequent attribution, in the scientific community's view, to land processes. Here we emphasize the distinction between global net terrestrial biosphere flux, and significant localized net terrestrial sinks or sources. The existence of the latter does not necessarily constrain the former. For example, some of the evidence for an enhanced terrestrial sink is based on the global terrestrial balance of carbon flux, requiring assumptions about the 
magnitude of a land-use term. Other results are spatially explicit and independent of such assumptions.

The idea of a missing sink for atmospheric $\mathrm{CO}_{2}$ initially arose when direct estimates of net $\mathrm{CO}_{2}$ release due to land-use change first became available (Houghton et al. 1983a,b). These estimates were based on theory linking conversion rates and regrowth dynamics to sources and sinks of $\mathrm{CO}_{2}$ from vegetation and soils (Moore et al. 1981). Prior to that time, the existence of a significant terrestrial term (either source or sink) was doubted (Broecker et al. 1979), and discussion was focused primarily upon atmosphere-ocean exchange as predicted by numerical models (see Section 2.2). The amount of fossil carbon emissions not sequestered by the oceans (the "airborne fraction") was observed to be slightly less than that predicted by most oceanatmosphere carbon models (Oeschger et al. 1975). Thus, there was a small residual sink, and claims of a large (3-6 Pg C yr ${ }^{-1}$ ) tropical source (Woodwell 1978) were largely ignored, or assumed to be balanced by regrowth (Broecker et al. 1979).

The basic approach was to implicitly calculate a residual flux using a method known as "deconvolution", in which the ocean models calculate. by difference, the amount of supplementary carbon required to reproduce the measured rates of atmospheric increase (Oeschger et al. 1975, Keeling et al. 1989a). The atmospheric increase was prescribed by ice core measurements and the direct record. The residual flux, calculated in this way, revealed a period of net emissions prior to ca. 1950 that were attributed to a "pioneer effect" of European colonization (and hence forest conversion) of the Western Hemisphere (e.g., Keeling et al. 1989a). After ca. 1950, the residual is negative-valued, corresponding to the small sink mentioned above. This sink was widely believed to be related to direct $\mathrm{CO}_{2}$ fertilization of the terrestrial biosphere, the magnitude of which was parameterized in models using a formulation known as the " $\beta$-factor" (Section 2.3.3). This formulation assumed (typically) a logarithmic 
dependence of the fertilization flux multiplied by a constant $(\beta \approx 0.3)$. There is a wealth of literature on this effect (Amthor 1995), including attempts to characterize $\beta$ at the process level: we will discuss this in more detail in the next section.

Subsequent to confirmation of a likely tropical net carbon source as revealed by independent biotic flux estimates (Houghton et al. 1985), tallying the carbon budget revealed two important facts: (1) the temporal pattern of the clearing-rate based estimates of net terrestrial flux were very different from the net residual (presumably of biospheric origin) deconvolved from the ocean carbon models and atmospheric data; and (2) the most reliable estimates of global oceanic uptake could not account for the $\mathrm{CO}_{2}$ which did not remain in the atmosphere; only ocean models that had unrealistically high uptake rates could balance the budget. At the same time, it was generally assumed that the undisturbed biosphere was in net balance. Thus, it was clear that key processes were being ignored, or incorrectly described, and the controversial search for "missing" carbon $\left(\sim 1-3 \mathrm{Pg} \mathrm{yr}^{-1}\right)$ was initiated.

After more than ten years of research, considerable controversy about the global carbon cycle remains, but it is now widely believed that the unaccounted-for carbon has been (for the most part) stored on land. There are two lines of reasoning for this that have evolved somewhat independently, and they will be discussed below. First, evidence from atmospheric/oceanic data and models indicate a net flux of $\mathrm{CO}_{2}$ into the terrestrial biosphere that is unrelated to tropical land-use change. Spatially explicit analyses of the same type (e.g., using atmospheric transport models) indicate that the sink resides in the northern high latitudes. Second, several physiological and ecological mechanisms for enhanced terrestrial carbon uptake have been identified which could potentially account for more than the imbalance. Conversely, no additional oceanic biogeochemical or physical carbon sink mechanisms have been identified. Indeed, there seems to be a convergence in the oceanographic community on a 
relatively stable value of net oceanic carbon sink $\left(\sim 2 \mathrm{Pg} \mathrm{yr}^{-1}\right)$. Both arguments above are consistent with one another because the terrestrial mechanisms in question are likely to be operating most significantly in the mid-to-high latitudes of the Northern Hemisphere.

\subsubsection{Evidence from atmospheric and oceanic measurements and modeling}

Atmospheric data can provide information about the patterns of carbon sources and sinks at the lower (land and ocean) boundary. Carbon dioxide in the atmosphere is chemically inert. Thus, the dynamics of the state of atmospheric $\left[\mathrm{CO}_{2}\right]$ are governed by surface sources and sinks, and three-dimensional transport. In addition, other tracers (e.g., isotopes of carbon, or $\mathrm{O}_{2}$ ) are associated with carbon cycle processes, and their transformation amongst major reservoirs may be chemically or physically different from $\mathrm{CO}_{2}$. These constituents can provide additional constraints on the carbon cycle if the differentiation is correctly described. Research that accounts for atmospheric transport, and/or composition has led to a better understanding of the complexity and importance of regional-scale processes on land in the carbon budget.

Attention was first dramatically focused upon the terrestrial biosphere when Tans et al. (1990), based on geographically specific observations and modeling, made the provocative statement that "a large amount" (2-3 $\left.\mathrm{Pg} \mathrm{C} \mathrm{yr}^{-1}\right)$ was currently being taken up by continental ecosystems in the Northern Hemisphere. This result was surprising because the sink was inconsistent both in magnitude and spatial orientation with the small hypothesized direct $\mathrm{CO}_{2}$ fertilization effect predicted by the ocean models. In addition, the result was independent of assumptions about a (disputed) net source from the tropical forests. Instead, like most calculations of this variety, assumptions about the ocean mechanisms were most critical. Tans et al. (1990) combined atmospheric and oceanic measurements of the partial pressure of $\mathrm{CO}_{2}$ with a model of atmospheric transport and prescribed spatial distribution of fossil carbon 
sources. They used the transport model to calculate the latitudinal gradient of $\mathrm{CO}_{2}$ given the prescribed boundary conditions (ocean exchange and fossil fuel input). Their analysis resulted in a modeled north-south gradient of atmospheric $\mathrm{CO}_{2}$ that was steeper than observed. They concluded that the observed gradient could be maintained only by a high-latitude sink over land because the prescribed oceanic flux in the Northern Hemisphere would not support a high enough rate of $\mathrm{CO}_{2}$ removal from the atmosphere. Possible mechanisms for enhanced terrestrial uptake were not known; various parameter combinations attempted to represent $\mathrm{CO}_{2}$ fertilization, and they produced unrealistic results.

Some concerns were raised about the accuracy of the Tans et al. (1990) calculation (e.g., Siegenthaler and Sarmiento 1993) mainly because of problems with their calculation of global oceanic $\mathrm{CO}_{2}$ flux, which was may have been as much as $\mathrm{I} \mathrm{PgC} \mathrm{yr}^{-1}$ too low. Siegenthaler and Sarmiento (1993) point out three considerations that would lead to a revision of the Tans et al. (1990) ocean flux: (1) skin temperature should be used instead of bulk temperature in extrapolating global ocean partial pressure of $\mathrm{CO}_{2} ;(2)$ the fraction of the riverine flux $\left(\sim 0.8 \mathrm{PgC} \mathrm{yr}^{-1}\right)$, not deposited as sediments and balanced by ocean outgassing, should be accounted for; and (3) the carbon monoxide that is transported from the northern to Southern Hemisphere should be included in the calculation of the interhemispheric gradient. This revision, which brings the atmosphere-to-ocean flux into general agreement with model estimates (Siegenthaler and Oeschger 1987, Sarmiento and Siegenthaler 1992), does not change the general conclusions related to the strength of the interhemispheric gradient. Moreover. a more modest-sized enhanced terrestrial sink in the Northern Hemisphere would bring the Tans

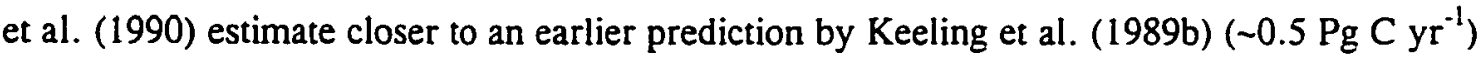
which utilized simple terrestrial and ocean box models coupled to a three dimensional atmospheric model. In the Keeling et al. (1989b) analysis, model parameters were adjusted 
under various assumptions about terrestrial activity in order to achieve a best fit to the latitudinal $\left[\mathrm{CO}_{2}\right]$ distribution. Their results were consistent with a greater "fertilization effect" in the high latitudes than in the tropics.

The two analyses above utilized multiple realizations of modeled atmospheric transport, and as such were not formal inversions of the model. Work by Enting and Mansbridge $(1989,1991)$, however, pioneered the use of formal inversions for the investigation of surface source/sink distributions. These calculations utilized two-dimensional atmospheric transport models (vertically and latitudinally resolved), parameterized from full 3-D forward simulations. Model inversion techniques, in this context, calculate the carbon flux at the lower boundary required to produce atmospheric $\mathrm{CO}_{2}$ concentrations that are most consistent with the data (interpolated in space and time on to the model grid). The results of Enting and Mansbridge (1991) indicate a rather large net sink $\left(\sim 2 \mathrm{Pg} \mathrm{yr}^{-1}\right)$ for carbon in approximately the 50-70N latitudes. A portion of this flux is likely caused by North Atlantic deep-water formation, but they argue that "it seems necessary" to attribute some of it to terrestrial biotic processes, based on the disproportionate observation of northern and southern oceanic uptake.

The isotopic composition of carbon in atmospheric $\mathrm{CO}_{2}$ can aid exploration of the global carbon budget. In particular, the heavy isotope ${ }^{13} \mathrm{C}$ is preferentially fractionated against in the formation of organic matter (by photosynthesis) but only weakly so by the ocean. Thus, combustion of fossil carbon and biospheric destruction lead to dilution of ${ }^{13} \mathrm{C}$ in the atmosphere. The amount of ${ }^{13} \mathrm{C}$ in a reservoir is expressed as the ratio of ${ }^{13} \mathrm{C}$ to ${ }^{12} \mathrm{C}$ relative to a standard ratio:

$$
\delta^{13} \mathrm{C}=\left[\frac{\left({ }^{13} \mathrm{C} /{ }^{12} \mathrm{C}\right)_{\text {sample }}}{\left({ }^{13} \mathrm{C} /{ }^{12} \mathrm{C}\right)_{\text {sandard }}}-1\right] \cdot 1000
$$


Keeling et al. (1989a) used this quantity, taken from direct atmospheric measurements, to add one degree of freedom to their model deconvolution ("double deconvolution") of the residual $\mathrm{CO}_{2}$ flux. In this way they were able to partition the residual into an oceanic and terrestrial component. It was observed that (1) the land biosphere and the oceans exhibit strong interannual variability related to natural climate cycles, (2) the two flux anomalies are generally opposite in sign, and (3) the amplitude of the terrestrial term is somewhat greater. However, it is difficult to compare their analysis to budget calculations because of model assumptions about the ocean and particularly the terrestrial biosphere, particularly the a priori inclusion of a "fertilization" factor.

Based only on atmospheric and oceanic measurements of the change in $\left[\mathrm{CO}_{2}\right]$ and $\delta^{13} \mathrm{C}$ from 1970-90, Quay et al. (1992) determined that the terrestrial biosphere as a whole provided essentially no net flux of $\mathrm{CO}_{2}$ to/from the atmosphere during that period. Using a mass-balance approach, they solved a set of equations that describe the rate of change of ${ }^{13} \mathrm{C}$ and ${ }^{12} \mathrm{C}$ in the major reservoirs over the period 1970-90. The ocean data consisted of depth-integrated dissolved inorganic carbon at a number of fixed stations, and $\delta^{13} \mathrm{C}$ measured along two Pacific transects. This information, combined with specification of fossil fuel emissions over the same period, allowed for a determination of the partitioning between terrestrial and oceanic uptake of the excess carbon. They found that of the $43 \%$ of emissions not remaining in the atmosphere, $42 \%$ was sequestered by the ocean over this period, implying a net-neutral biosphere from 1970-90. Their result is "model independent", in the sense that it does not require assumptions about ocean or atmosphere transport. This conclusion further supported the idea that if a tropical source exists, it must be balanced by some other terrestrial mechanism(s). However, 
another perspective is that (according to this analysis) the existence of a high-latitude sink requires a tropical source.

Perhaps the most convincing evidence to date supporting a mid-to-high latitude northern hemispheric carbon sink is derived from a combination of isotopic and inversion methods. Ciais et al. (1995a) performed a model inversion on atmospheric $\left[\mathrm{CO}_{2}\right]$ and $\delta^{13} \mathrm{C}$ data from the Climate Monitoring and Diagnostics Laboratory sampling network (Conway et al. 1994). They accounted for lags in the terrestrial exchange of ${ }^{13} \mathrm{C}$ (the "isotopic disequilibrium") by using ecosystem component turnover rates and isotope discrimination from established terrestrial models: Century (Parton et al. 1987) and SiB (Sellers et al. 1986), respectively. They observed a net tropical source $\left(\sim 2 \mathrm{PgC} \mathrm{yr}^{-1}\right)$, but also a large net sink $\left(\sim 3.5 \mathrm{PgC} \mathrm{yr}^{-1}\right)$ in the northern mid-to-high latitudes. This estimate indicates both a net terrestrial sink, and a localized (continental) Northern Hemisphere sink $\left(\sim 1.5 \mathrm{Pg} \mathrm{C} \mathrm{yr}^{-1}\right)$ that are on the high end of all estimates to date, but the data used for this initial study were from 1992, the anomalously cool year following the eruption of Mt. Pinatubo. The authors note in a follow-up report (Ciais et al. 1995b) that the calculated sink estimate using 1993 data was still present, but substantially less. This is consistent with speculation that the Pinatubo anomaly resulted in enhanced net storage of $C$ on land in 1992 (Keeling et al. 1995; Chapter 4).

Recently, high-precision measurements of $\mathrm{O}_{2}$ concentration in the atmosphere have provided an additional constraint on global carbon budget (R. Keeling et al. 1996), analogous to that provided by isotopic measurements. Changes in the $\mathrm{O}_{2} / \mathrm{N}_{2}$ ratio are stoichiometrically linked to $\mathrm{CO}_{2}$ fluxes by the formation (via photosynthesis) and destruction (via respiration or combustion) of organic matter, with a $\mathrm{CO}_{2}: \mathrm{O}_{2}$ ratio of about 1.1. There is no such relationship associated with physical oceanic exchange because $\mathrm{CO}_{2}$ dissolution in the oceans proceeds via carbonate buffering and does not involve oxygen. R. Keeling et al. (1996) used measurements 
of $\mathrm{O}_{2} / \mathrm{N}_{2}$ and $\mathrm{CO}_{2}$ at a global network of sites. collected from 1991-94, to infer the partitioning of global atmospheric $\mathrm{CO}_{2}$ exchange between the oceans and the terrestrial biosphere. They concluded that the oceans and the biosphere sequestered roughly the same amount over that time: 1.7 and $2.0 \mathrm{PgC} \mathrm{yr}^{-1}$, respectively. The $\mathrm{O}_{2} / \mathrm{N}_{2}$-based results are quantitatively similar to $\delta^{13} \mathrm{C}$-based analyses of Ciais et al. (1995a,b); the two studies are directly comparable because the data used by R. Keeling et al. (1996) are from approximately the same time period (199194).

The Ciais et al. studies (1995a,b) and that of R. Keeling et al. (1996), though they generally confirm the notion of an extratropical carbon sink, highlight some aspects of the controversy over the carbon budget. This is because both analyses indicate a strong global terrestrial net sink in the early 1990s; whereas, the other studies mentioned above (for the 1980s) calculate, or implicate, a roughly net neutral biosphere. If all the results are reliable. then there are two possible resolutions to this issue: (1) land-use $\mathrm{CO}_{2}$ emissions declined markedly from the eighties to the nineties; or (2) there is significant interannual variability in the terrestrial component of the global carbon cycle. There is evidence for the latter (Keeling et al. 1989a, Schimel et al. In press a), but the former should not be discounted (Alves et al. In press).

The ostensible temperature dependence revealed by Ciais et al. (1995b) is also suggestive of a global feedback mechanism that has bearing on the discussion of the global carbon budget as well as climate. If it is true that terrestrial uptake processes (whatever their cause) operate less strongly during warm years, and if the Earth's temperature continues to rise, then those processes will remove less and less $\mathrm{CO}_{2}$ from the atmosphere. The resulting increase in atmospheric $\left[\mathrm{CO}_{2}\right]$ growth rate could further stimulate warming by modifying the atmospheric heat balance. This kind of positive feedback has been discussed often in the 
literature, but there are also negative feedbacks with climate that involve terrestrial as well as climate mechanisms. In Chapter 4 we will discuss climate-driven variability in the terrestrial carbon cycle, but we focus on longer time scale processes in this chapter, under the assumption of little or no transient climate impacts on the carbon cycle over the last 200 years.

\subsubsection{Terrestrial mechanisms capable of resulting in a net carbon sink}

A number of recent studies have investigated the effects (both direct and indirect) of anthropogenic activities on the rate of carbon storage by the terrestrial biosphere. The results of these analyses, which include modeling efforts, integrated model simulations with global data, and extrapolated field studies, have aroused considerable interest because of the relative consistency with which they suggest northern forest ecosystems (predominantly) as a recent significant net global sink of carbon. While the potential for these effects to be present is wellknown, their magnitude, exact spatial distribution, and degree of interaction are highly uncertain. This is because the uptake mechanisms operate at the plant or ecosystem level, and global estimates are extrapolated, often by using models. Below we briefly discuss the four mechanisms thought to be the most significant for storing excess carbon: direct $\mathrm{CO}_{2}$ fertilization, nitrogen fertilization, land-use change, and climate variability.

The effect of increased ambient $\left[\mathrm{CO}_{2}\right]$ levels on leaf photosynthesis is well-understood at the physiological level. There is a positive, hyperbolic (Michalis-Menton) relationship between $\left[\mathrm{CO}_{2}\right]$ and net photosynthesis which is primarily due to the kinetics of the enzyme ribulose-1,5-bisphosphate carboxylase-oxygenase (rubisco) in the $\mathrm{C} 3$ photosynthetic pathway (modification of $\mathrm{C} 4$ and CAM photosynthetic rates by increased $\left[\mathrm{CO}_{2}\right]$ is much lower because of their " $\mathrm{CO}_{2}$-concentrating" modes). The extent to which this physiological manifestation translates into whole plant, ecosystem, and especially global scale $\mathrm{CO}_{2}$ fertilization (net carbon 
storage) is subject to considerable uncertainty (Amthor 1995). This uncertainty arises primarily from (1) differences in patterns of resource availability between different native ecosystems, (2) differences between the observed response of individual leaves, plants. and ecosystems, and (3) differences between experimental and natural conditions (Oechel et al. 1994).

Various techniques have been used to study the effect of elevated $\left[\mathrm{CO}_{2}\right]$ on plants. A very common approach is to report the relative change in photosynthesis or biomass increment in response to a doubling of current ambient $\left[\mathrm{CO}_{2}\right]$ (e.g., $350 \mathrm{ppm}$ vs. $700 \mathrm{ppm}$ ). Many carbon dioxide fertilization studies have been carried out at the leaf/branch level using chambers. These studies result in estimates of short-term effects of $\mathrm{CO}_{2}$ on photosynthesis. Also, whole plant chambers have also been used, typically on plants in the laboratory, grown in pots under controlled environmental conditions. In the field, closed chambers (mini-greenhouses), and open-top chambers provide somewhat more realistic conditions, but chamber effects can be nevertheless quite large, owing to the very different aerodynamic conditions relative to the open air. Thus, some "free-air" $\mathrm{CO}_{2}$ enrichment studies have been performed, but they are difficult to maintain for an extended period, especially for large canopies. It is still true that very little is known about the long term ( $>1$ year) response of natural systems because of experimental difficulties and the potential complexity of ecosystem response (e.g., acclimation). Moreover, whole ecosystem studies are rare (Oechel et al. 1994).

Ecosystem level interactions are an important determinant of the expression of $\mathrm{CO}_{2}$ fertilization. Water-limited plants generally experience a greater enhancement because high ambient $\left[\mathrm{CO}_{2}\right]$ results in increased water use efficiency (i.e., water loss is lessened by reduction of the stomatal aperture). This moisture dependence is partly responsible for the conflicting results mentioned above. Nutrient feedbacks may be more complex, but it is generally true that nutrient limitation will lead to little or no growth enhancement; plant stoichiometric 
relationships between nutrient uptake and carbon assimilation are not entirely inflexible, but they are constrained. In addition, it may be true that ecosystems tend toward a nutrient-limited steady-state because of the coupling of plant production. litterfall, soil organic matter formation, and nutrient availability (see Chapter 4). Thus an initial positive response will likely be mitigated in the long-term. Modeling studies (Pierce et al. 1993, VEMAP 1995) and field experiments (e.g., Webber et al. 1994, Oechel et al. 1994; see review by Amthor 1995) have supported this view. In addition, allocation of biomass carbon may proceed differently due to other environmental factors. For example, Holland et al. (1994) showed that increased production in one grassland ecosystem was allocated to roots which turn over rapidly: thus there was little or no long term carbon storage. Thus, process models and field experiments indicate that a "steady-state" enhancement of ecosystem production due to elevated $\left[\mathrm{CO}_{2}\right]$ are likely to be less than indicated by the initial response of plants.

At the global scale, direct $\left[\mathrm{CO}_{2}\right]$ fertilization was incorporated early-on in global carbon cycle modeling as a possible sink mechanism. Indeed, it was often assumed to be responsible for all of the residual carbon flux in global models (Bacastow and Keeling 1973, Keeling et al. 1989a, Moore and Braswell 1994, Friedlingstein et al. 1995). Discussion has centered around the representation of the dependence of enhanced terrestrial carbon storage as a function of $\left[\mathrm{CO}_{2}\right]$, i.e., the $\beta$-factor, mentioned above. Aggregated carbon cycle models that are constrained by atmospheric measurements and process-based ecosystem models (parameterized with generalized response factors from experiment results) both indicate that

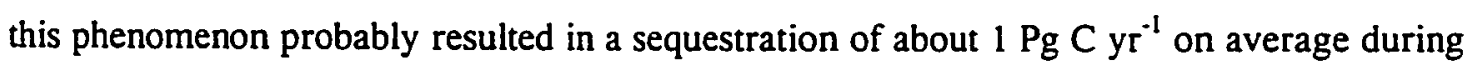
the decade of the 1980s (as noted by the IPCC; Schimel et al. 1995).

Because most terrestrial systems are N-limited (Vitousek and Howarth 1991), it is possible that increasing $\mathrm{N}$ deposition derived from fossil fuel burning and agriculture is 
stimulating terrestrial production. An early estimate of this effect was made by Peterson and Melillo (1985); using stoichiometric arguments, they calculated that approximately $0.2{\mathrm{Pg} \mathrm{C} \mathrm{yr}^{-}}^{-}$ ${ }^{1}$ was sequestered by forests, rivers, and the coastal ocean in 1980 . Townsend et al. (1996;

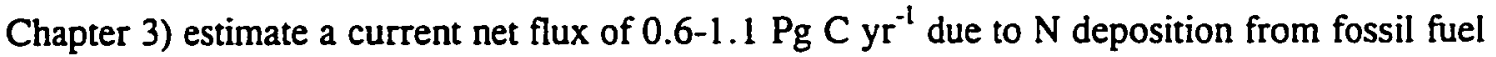
derived $\mathrm{NO}_{\mathrm{y}}$ alone, using deposition estimates provided by an atmospheric chemistry/transport model. Their estimate takes into account the spatial distribution of vegetation and deposition, as well as cumulative effects. The inclusion of other sources of $\mathrm{NO}_{\mathrm{x}}$ as well as $\mathrm{NH}_{\mathrm{x}}$ will drive that estimate higher (Holland et al, In press), but it is clear that there is a limit to how long terrestrial systems will remain $\mathrm{N}$-limited under increasing loads of deposition (Aber et al. 1989). These feedbacks (arising from acidification and high ozone levels) are not well understood globally, but it is clear they will mitigate the $\mathrm{N}$-fertilization to some extent. See Chapter 3 for a more detailed discussion of the impacts of nitrogen and other pollutant emissions on terrestrial carbon.

Another possible sink mechanism is related to changing land-use instead of changing plant resource-use efficiencies. It is clear that widespread abandonment of agricultural lands has occurred in North America (e.g., Houghton 1993a). These lands were first cultivated prior to the industrial revolution (i.e., the "pioneer effect"), and increasing regrowth relative to destruction in this region would result in a net storage of carbon, at least for some period of time. Two recent studies have used forest survey information in conjunction with assumed growth rates to calculate a carbon balance for northern forests. Kauppi et al. (1992) and Sedjo (1992) estimated, respectively, that $0.85-1.2 \mathrm{Pg} \mathrm{yr}^{-1}$ and $\sim 0.7 \mathrm{Pg} \mathrm{yr}^{-\mathrm{l}}$ of carbon has been stored by northern temperate and boreal ecosystems. In contrast, it appears that there may be substantial deforestation currently underway in the Russian temperate zone (Melillo et al. 1988). Thus, potential carbon storage due to Northern Hemisphere forest regrowth is subject to 
considerable debate, and an evaluation of the available literature suggests that it may currently (during the 1980s) account for 0-1 Pg $\mathrm{yr}^{-1}$ (Houghton 1995, Schimel 1995b).

Climate variability may affect terrestrial carbon cycling on time scales relevant to the global carbon budget calculation. Dai and Fung (1993) used a simple model of net terrestrial exchange to suggest that variations in temperature and precipitation (from 1940 to 1989) could have accounted for a net sink over those decades. The net storage resulted from a differential increase of production versus decomposition for a large number of ecosystems, and in particular, northern forests. This effect was due to the combined temperature and moisture dependence of the two competing processes. Direct and indirect effects of climate on terrestrial biogeochemistry are complex, however, and it has been argued that no systematic relationships are apparent at the global scale, at least to the extent that an estimate of climate change induced fluxes for the decade of the 1980s can be made (Schimel 1995b). Moreover, it is very difficult to separate natural from anthropogenic climate variability at present. Nevertheless, we argue that the issue of climate- $\mathrm{CO}_{2}$ interaction as mitigated by terrestrial ecosystems, though complex and somewhat separate from budget considerations, is a tractable problem, and one that is very important for understanding many carbon cycle issues (Chapter 4).

All of the above mechanisms (including $\mathrm{CO}_{2}$ fertilization; see Friedlingstein et al. 1995) have in common the fact that they are likely to be distributed most prominently in the northern temperate-to-boreal latitudes. Guided by results from the atmospheric measurement/modeling community, this pattern has become a virtual prerequisite for proposed terrestrial sinks (not associated with tropical land-use). Because both interaction (enhancement) and overlap (double counting) between many of the above mechanisms is possible, it is probable that they are at least partially additive. Thus they could account for as much as $1.9 \mathrm{Pg}$ C $\mathrm{yr}^{-1}$ (Schimel 1995b), slightly more than the "missing sink". While there is no carbon that is 
literally missing, it is still true that none of the terrestrial terms in the budget are well quantified. Future work will necessarily involve narrowing the uncertainties and understanding possible feedbacks so that reliable projections of future atmospheric $\left[\mathrm{CO}_{2}\right]$ can be made.

Research leading to conclusions about the existence of a net terrestrial sink has not resulted in a more precise explication of the carbon budget. Indeed, the total uncertainty has increased with the inclusion of new terms, as we will discuss later. The original question of the cause of the missing sink, though it has been qualitatively answered, has led to more questions about the global carbon cycle and its interaction with climate and other biogeochemical cycles. The cumulative advance in understanding has forced a shift in current thinking toward reducing uncertainty in the magnitude, spatial patterns, and temporal variability of known carbon cycle processes, particularly within the terrestrial biosphere.

\subsubsection{Analysis of the uncertainty in the carbon budget}

We can describe the anthropogenically perturbed global carbon budget, including all the terms presented in the last section, with the following equation:

$$
\Delta A=F+D+O+R+C+N+\varepsilon_{1}
$$

where $\Delta A$ is the atmospheric increase, $F$ is the fossil emissions, $O$ is the net oceanic flux, $R$ is uptake by Northem Hemisphere forest regrowth, $\mathrm{C}$ is direct $\mathrm{CO}_{2}$ fertilization, $\mathrm{N}$ is nitrogen fertilization, and $\varepsilon_{1}$ is the residual effect of all other processes. Current estimates for values of the terms, taken from the literature, are given in Table 2.1. In this representation, the atmosphere is given central importance as an integrator. Indeed, it is the atmospheric measurements that have contributed most to our understanding of the carbon cycle, and it acts 
Table 2.1 The average annual budget of anthropogenic $\mathrm{CO}_{2}\left(\mathrm{Pg} \mathrm{C} \mathrm{yr}{ }^{-1}\right)$ for the period 19801989 , based on Schimel (1995b) and IPCC (1995), but revised to show the ranges as fuzzy numbers, given varied levels of confidence. Positive numbers indicate a positive increment in the atmosphere. Errors are summed quadratically in the first column; in the remaining columns, residuals are represented using fuzzy arithmetic.

\begin{tabular}{|c|c|c|c|c|}
\hline Carbon budget term & & I & II & III \\
\hline Storage in the atmosphere $(\Delta A)$ & $3.2 \pm 0.2$ & {$[3.0,3.4]$} & {$[3.0,3.4]$} & {$[3.0,3.4]$} \\
\hline Fossil emissions $(F)$ & $5.5 \pm 0.5$ & {$[5.0,6.0]$} & {$[5.0,6.0]$} & {$[5.0,6.0]$} \\
\hline $\begin{array}{l}\text { Net emissions from land-use } \\
\text { change in the tropics }(D)\end{array}$ & $1.6 \pm 1.0$ & & {$[0.6,2.6]$} & {$[0.6,2.6]$} \\
\hline Oceanic uptake $(O)$ & $-2.0 \pm 0.8$ & & {$[-2.8,-1.2]$} & {$[-2.8,-1.2]$} \\
\hline $\begin{array}{l}\text { Uptake by Northern Hemisphere } \\
\text { forest regrowth }(R)\end{array}$ & $-0.5 \pm 0.5$ & & & {$[-1.0,0.0]$} \\
\hline $\mathrm{CO}_{2}$ fertilization $(C)$ & $-1.0 \pm 0.5$ & & & {$[-1.5,-0.5]$} \\
\hline $\mathrm{N}$ deposition $(N)$ & $-0.6 \pm 0.3$ & & & {$[-0.9,-0.3]$} \\
\hline Residual $\left(\varepsilon, \varepsilon_{1}, \varepsilon_{2}, \varepsilon_{3}\right)$ & $0.2 \pm 2.0$ & {$[-3.0,-1.6]$} & {$[-4.4,0.6]$} & {$[-3.6,4.0]$} \\
\hline
\end{tabular}

as a hub through which the other components interact. As we have seen, the terms $F, D, O$, and $\Delta A$ have received the most careful attention to date, and they do not overlap. Thus they may be distinguished (subjectively) from the terms $R, C$, and $N$, which likely interact, and which are less understood, in principle. Further, we can distinguish the fossil fuel flux and the atmospheric increase ( $F$ and $\Delta A$ ), which are known to within approximately $10 \%$, from the oceanic and tropical land-use fluxes because the latter are derived using models and data whose uncertainty arises primarily from spatial extrapolation. Thus:

$$
\Delta A=F+D+O+\varepsilon_{2},
$$

$\Delta A=F+\varepsilon_{3}$. 
The distinction made in Equations 2.2-2.4 are convenient for a number of reasons. First, it divides the uncertainty into levels of presumption, a concept encountered in the addition of "fuzzy" numbers (see Kaufmann and Gupta 1985). Fuzzy arithmetic is based on an extension of the confidence interval, and is a way of dealing with quantities that are intrinsically uncertain; we will borrow the simple concept of addition and subtraction of fuzzy numbers. Second, there is a conceptual separation: Equation 2.3 describes the rate of change in $\left[\mathrm{CO}_{2}\right]$ due to the only exogenous anthropogenic flux, fossil fuel combustion, plus all other mechanisms, both terrestrial and oceanic. Equation 2.4 includes the relatively well understood (but still quite uncertain) deforestation and ocean net exchange. Thus the residuals, in each case, represent the balance of fluxes not accounted for that exist below a (loosely defined) threshold of confidence.

The last three columns of Table 2.1 show the estimated values for Equations 2.2-2.4 as fuzzy numbers. The residuals in each case yield some insight into the historic progression of the understanding of the anthropogenic carbon budget, because (as discussed above) the ocean was considered first as the primary mechanism for the removal of fossil $C$ that had not remained "airborne"; then the deforestation fluxes began to be incorporated into models of varying complexity; finally, other terrestrial mechanisms have been added to the list. We can see that the range of uncertainty has increased as the important carbon cycle mechanisms have

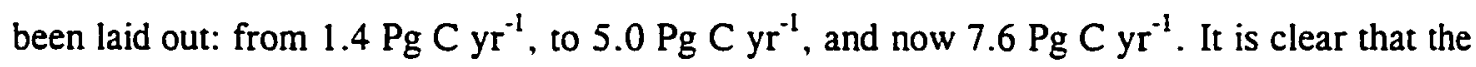
numbers and ranges themselves are subject to uncertainty, and other mechanisms. both oceanic and terrestrial, may exist that have not been identified. 


\subsection{The Lifetime of Excess Atmospheric $\mathrm{CO}_{2}{ }^{+}$}

\subsubsection{Introduction}

As we have discussed, the increase in the atmospheric $\mathrm{CO}_{2}$ concentrations (as well as other radiatively active trace gases) due to human activity has produced serious concern regarding the heat balance of the global atmosphere. Specifically, the increasing concentrations of these gases will lead to an intensification of the Earth's natural greenhouse effect (Shine et al. 1990, Watson et al. 1990,1992, Isaksen et al. 1992). Shifting this balance will force the global climate system in ways which are not well understood, given the complex interactions and feedbacks involved, but there is a general consensus that global patterns of temperature and precipitation will change, though the magnitude, distribution and timing of these changes are far from certain. The results of general circulation models indicate that globally averaged surface temperatures could increase by as much a $1.5^{\circ}-4.5^{\circ} \mathrm{C}$ (e.g., Mitchell et al. 1990, Gates et al. 1992) in a world with an atmospheric concentration of $\mathrm{CO}_{2}$ twice that of the preindustrial period (i.e., roughly 550-580 ppm).

The uncertainty of future climate change does not rest solely on issues of physicalclimate system dynamics and their representation in general circulation models. Understanding the carbon cycle (Fig. 2.3) is a key to comprehending the changing terrestrial biosphere and to developing a reasonable range of future concentrations of carbon dioxide and other greenhouse gases (e.g., Bacastow and Keeling 1973, Bolin et al. 1979, Björkstrom 1979, Bolin 1981, Moore 1985, Schlesinger 1991, Bolin and Fung 1992). Conversely, predictions about the physical-climate system and climate change are confounded by the fact that the carbon cycle is still not adequately understood or quantified globally. Some uncertainty remains about the role of the oceans in carbon dioxide exchange; they are without question a sink for anthropogenic

${ }^{\dagger}$ Adapted from the article by Berrien Moore III and B.H. Braswell, Global Biogeochemical Cycles 8:2338, 1994. Copyright by the American Geophysical Union. 
$\mathrm{CO}_{2}$, but the strength of this sink is somewhat unclear (e.g., Keeling et al. 1989a, Tans et al. 1990, Sarmiento 1991. Siegenthaler and Sarmiento 1993. Sarmiento 1993).

Uncertainty also centers on the role of terrestrial ecosystems (e.g., Section 2.2.2), in which at least two factors govern the level of carbon storage. First and most obvious is the anthropogenic alteration of the Earth's surface, such as through the conversion of forest to agriculture, which can result in a net release of $\mathrm{CO}_{2}$ to the atmosphere. Second, and more subtle, are the possible changes in net ecosystem production (and hence carbon storage) resulting from changes in atmospheric $\mathrm{CO}_{2}$, other global biogeochemical cycles, and/or the physical-climate system. Ultimately, to adequately address such changes will require a much clearer understanding of the nitrogen and phosphorus cycles, since they are the limiting nutrients in most terrestrial ecosystems, but our knowledge of the way these biogeochemical cycles relate to the carbon cycle compares poorly with our general understanding of the individual cycles themselves. In sum, these uncertainties are reflected in our uncertainty about the atmospheric lifetime of excess $\mathrm{CO}_{2}$ (e.g., Watson et al. 1990,1992).

The lifetime of a trace atmospheric constituent may thought of as the amount of time required for some significant portion of an excess quantity of the gas to be removed chemically or to be redistributed to another part of the Earth system. The uncertainty in the distribution of sources and sinks for carbon dioxide (and for other greenhouse gases such as methane) makes a determination of atmospheric lifetime difficult and ambiguous. Unfortunately, the lifetime for $\mathrm{CO}_{2}$ (as well as for the other radiatively important gases) is important to know and particularly valuable in the policy context; it is necessary for the calculation of greenhouse warming potentials (GWP) (Lashof and Ahuja 1990, Watson et al. 1990, Maier-Reimer and Hasselmann 1987). 
The GWPs provide an index of the relative climatic impacts (costs) of the various greenhouse gases. The index takes into account the instantaneous radiative forcing of a single molecule $\left(a_{i}\right)$ and the response $c(t)$ of the system (atmospheric concentration as a function of time) to an instantaneous injection of gas:

$$
G W P_{i}=\frac{\int_{0}^{\infty} a_{i} \cdot c_{i}(t) d t}{\int_{b}^{\infty} a_{r} \cdot c_{r}(t) d t},
$$

where the subscript $r$ refers to the reference molecule. The curves $c_{i}(t)$ are generally computed using geochemical box-models. If the reference molecule is $\mathrm{CO}_{2}$, and the radiative forcing is taken to be equal to one (i.e., all $a_{i}$ 's are relative to $\mathrm{CO}_{2}$ ), then the denominator becomes:

$$
T_{\text {res }}=\int_{0}^{\infty} c(t) d t
$$

In an idealized context, where the concentration curve decays exponentially, $c(t)=a \cdot \exp (-t / \tau)$, then the integral (2.6) is equal to $\tau$, which is simply the length of time required for $c(t)$ to decline from any value along its trajectory to $1 / e$ of that value. Hence $\tau$ is often called the $e$ folding time. Generally, $c(t)$ is not strictly exponential, so the integral itself is called the residence time $\left(T_{\text {res }}\right)$. Given the convenient assumption that $c(t)=a \cdot \exp (-t / \tau)$, one often approximates the relaxation of the concentration by a weighted sum of exponentials (cf. Section 2.3.6).

We will show that calculating the integral (2.6) presents two types of difficulties. First, most model-derived estimates of the relaxation of the concentration of $\mathrm{CO}_{2}$ reveal a function 
which is not always well approximated by weighted sums of exponentials, even though these are often used to calculated $T_{\text {res. }}$. Second, the function $c(t)$ is quite sensitive to assumptions about the terrestrial biosphere and the relaxation experiment. We turn our attention to the latter difficulty first.

\subsubsection{A simple global carbon cycle model}

From the most elementary viewpoint, and focusing solely upon $\mathrm{CO}_{2}$, the global carbon cycle can be treated as a one box atmosphere linked to a submodel of terrestrial carbon dynamics and to an ocean carbon submodel. This is precisely the representation we employ in our experiments.

\section{The ocean carbon sub-models}

The net exchange of carbon between the atmosphere and the oceans is determined to a great extent by the carbonate chemistry of the upper mixed layer, the convective transport of dissolved carbon in sea water, the diffusion of carbon dioxide across the air-sea boundary, and the sinking of detrital carbon originating from the biological production of marine organisms (Bolin 1981, Broecker and Peng 1982, Moore 1985, Sarmiento 1991). All of these processes govern the exchange of carbon dioxide between the sea surface and the atmosphere, and all have been represented in models to varying extents; however, all do not play an essential role in the perturbation problem posed by the increase in carbon dioxide (Broecker 1991, Sarmiento 1991).

We investigate the effect of using different, simple representations of ocean carbon dynamics on the atmospheric concentration of $\mathrm{CO}_{2}$ using three atmosphere-ocean box models either standing alone or in a global carbon model which includes a terrestrial component. For a 
discussion of more complex models, see Maier-Reimer and Hasselmann (1987), Keeling et al. (1989a), and Maier-Reimer (1993). The submodels are:

1. Box-Diffusion Model (BD; Fig. 2.4): the turnover of carbon below 75 meters is represented by a diffusion equation. A constant coefficient of diffusivity is estimated to match an idealized profile of natural ${ }^{14} \mathrm{C}$ or bomb ${ }^{14} \mathrm{C}$ (Oeschger et al. 1975).

2. Outcrop-Diffusion Model (OC; Fig. 2.5): allows direct ventilation of the intermediate and deep oceans at high latitudes by incorporating outcrops for all sublayers into the box-diffusion formulation (Siegenthaler 1983). It is essentially the box-diffusion model with the addition of direct connections between the atmosphere and the deeper ocean layers. In a similar manner, a constant coefficient of diffusivity is based on natural ${ }^{14} \mathrm{C}$ or bomb ${ }^{14} \mathrm{C}$.

3. Twelve-Box Model (12B; Fig. 2.6): the Atlantic and Pacific-Indian Oceans are each divided into surface, intermediate, deep and bottom water compartments. The Arctic and Antarctic Oceans are divided into surface and deep water compartments. The model is calibrated against multiple tracer distributions (Bolin et al. 1983).

The three models have much in common: they are all diagnostic rather than prognostic; each uses carbon-14 in the parameterization process (in fact, carbon-14 is the basic clock for all of the models and hence controls much of their response); each includes ocean carbon chemistry (buffer or Revelle factor); and they all include some form of ocean mixing.

There are, however, some major differences. Ocean biology is explicitly included in only one (12B), whereas, it is simply incorporated into the parameterization of the diffusive process in both the BD and $\mathrm{OC}$ models. As far as transport is concerned, in the box-diffusion and outcrop-diffusion models all of the physics is captured by a single constant eddy diffusivity term; whereas, the Bolin et al. (1983) model (12B) has both advection and eddy diffusivities. Deep water formation (the sinking of cold, $\mathrm{CO}_{2}$-rich water at high latitudes) is not explicitly 


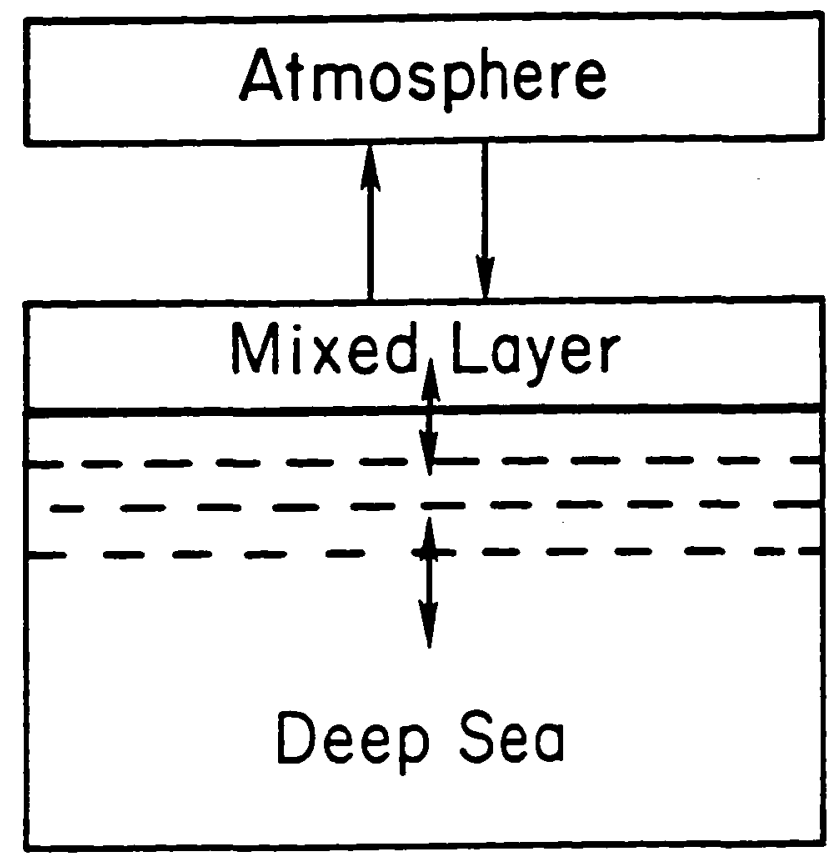

Figure 2.4 Box-diffusion model. The turnover of carbon below $75 \mathrm{~m}$ is represented by a diffusion equation. A constant coefficient of diffusivity is estimated to match an idealized profile of ${ }^{14} \mathrm{C}$ (Oeschger et al. 1975).

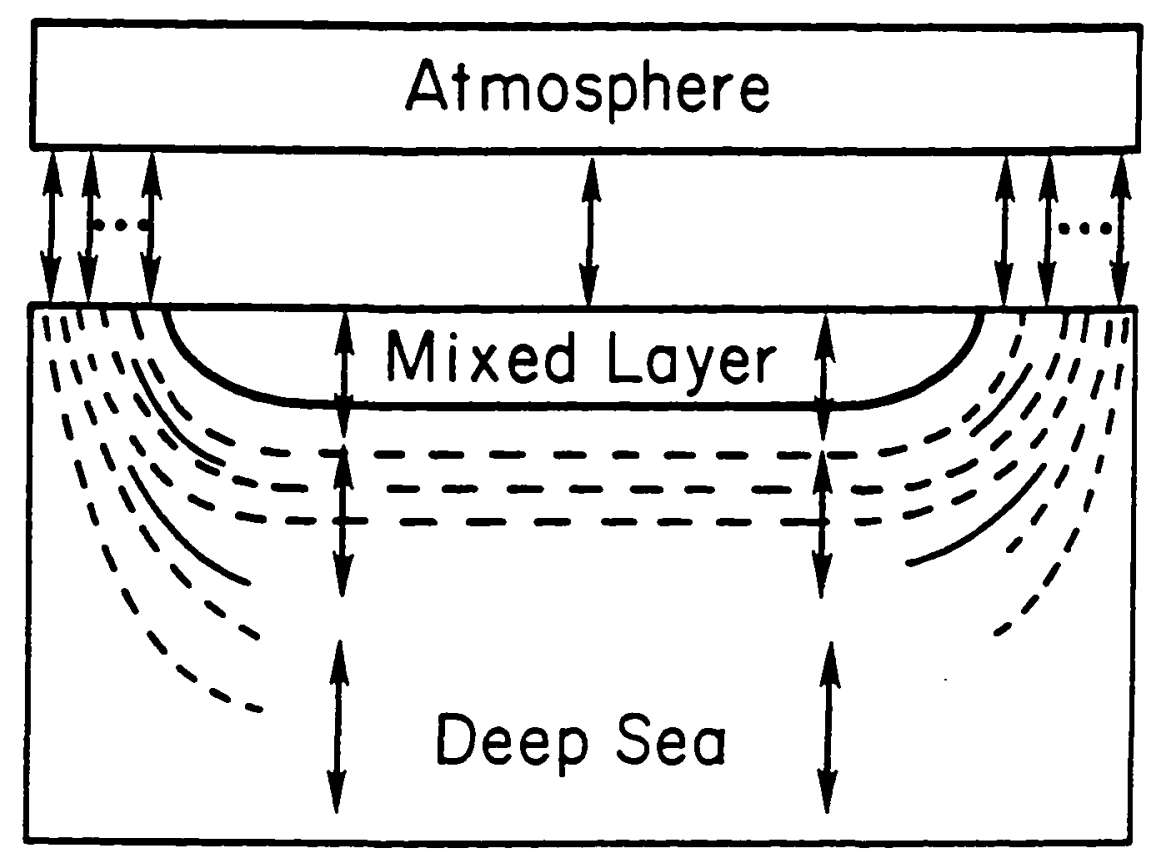

Figure 2.5 Outcrop-diffusion model. Direct ventilation of the intermediate and deep oceans at high latitudes is allowed by incorporating outcrops for all sublayers into the box-diffusion formulation (Siegenthaler 1983). 


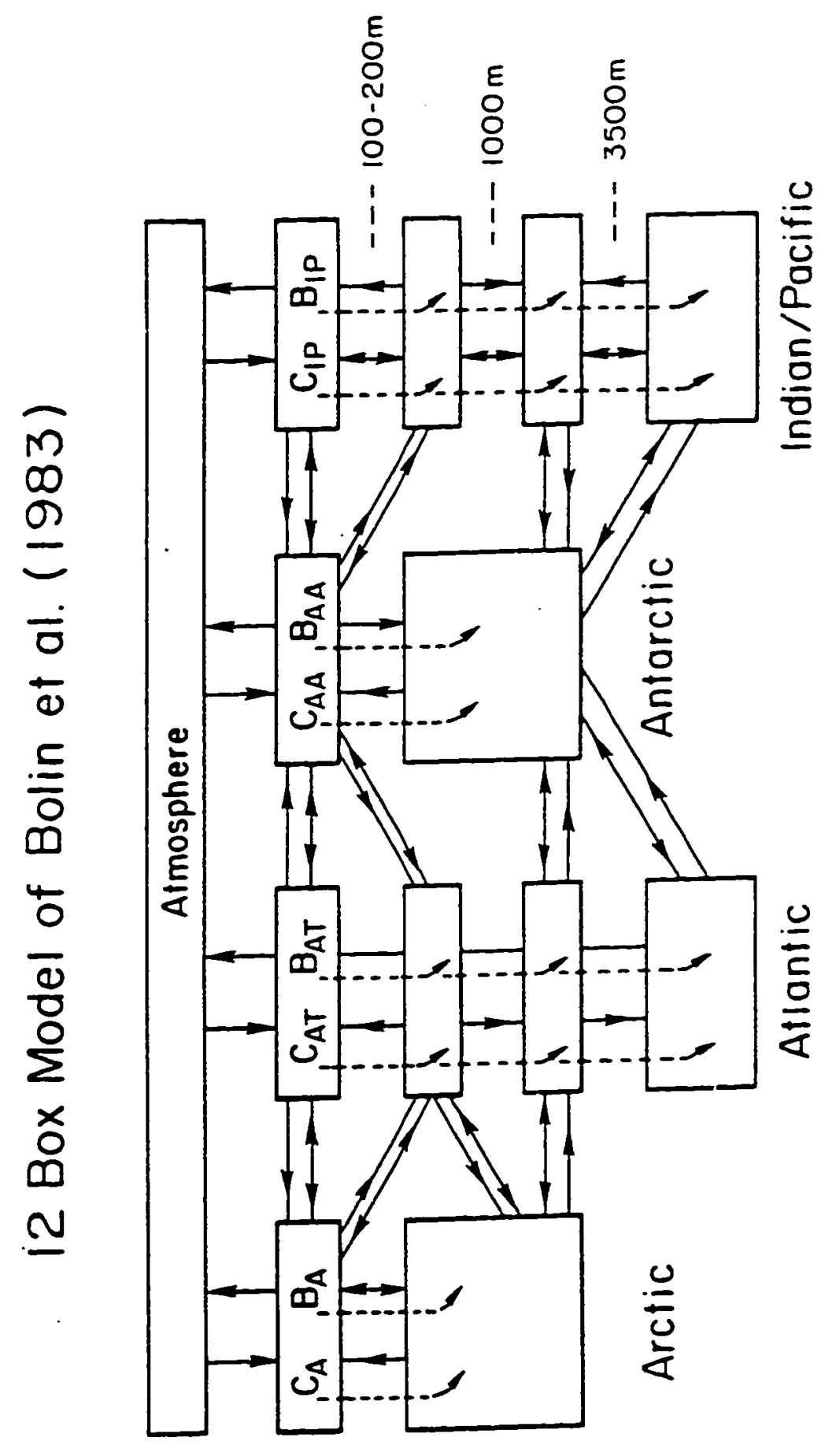

Figure 2.6 Twelve-box model. The Atlantic and Pacific-Indian oceans are each divided into surface, intermediate, deep, and bottom water components. The Arctic and Antarctic oceans are divided into surface and deep water components. The soft tissue and carbonate formation in surface boxes is indicated by $B$ and $C$, with appropriate subscripts for region, and the fluxes to and decomposition in deeper layers are indicated by the vertical dashed line and the branching solid arrow, respectively. 
considered in the box-diffusion model. The treatment of ocean chemistry also varies in complexity: the 12B model includes a detailed handling of the carbonate-borate equilibrium system; the BD model uses a constant buffer factor; and the OC model employs a quadratic that is a fit to measured data. Perhaps most importantly, the spatial configurations are quite different, as can be seen in Figs. 2.4-2.6. In addition, there are a host of smaller differences, including the specification of ocean volume and surface area, parameterization procedures, and carbon-14 profiles.

As a result of these differences, we find a range of responses to the two simplest experiments that can be performed with these three atmosphere-ocean submodels: a calculation of the perturbation response to a forcing by fossil fuel $\mathrm{CO}_{2}$ alone $\left(F_{F E} ; \mathrm{Fig} .2 .7\right)$ and to the forcing by $\mathrm{CO}_{2}$ from land-use change as well as the fossil fuel-derived flux $\left(F_{B}+F_{F E} ; \mathrm{Fig}\right.$. 2.8). A principal reason why all the models are so similar is the over-arching importance of ${ }^{14} \mathrm{C}$ in setting the basic rates within the models (Moore 1992); an interesting discussion of the ${ }^{14} \mathrm{C}$ constraints on ocean uptake of $\mathrm{CO}_{2}$ is provided by Broecker and Peng (1993). The OC model is the most efficient in taking up $\mathrm{CO}_{2}$, and this is the result of the instantaneous flux of $\mathrm{CO}_{2}$ to deep layers. In general, however, all of the ocean-atmosphere models lie underneath the data when forced by $F_{F E}$ alone and overshoot the record when both $F_{F E}$ and $F_{B}$ are used. Since there is evidence, based on historic reconstructions (Houghton et al. 1983a,b, Houghton and Skole 1990, Houghton 1991, Houghton et al. 1991, Skole and Tucker 1993), of an anthropogenically induced flux from the biosphere to the atmosphere, the discrepancy between model and data in Fig. 2.8 has given rise to the notion of a "missing carbon sink" (discussed earlier in Section 2.2). This is part of the motivation for considering the terrestrial component. 


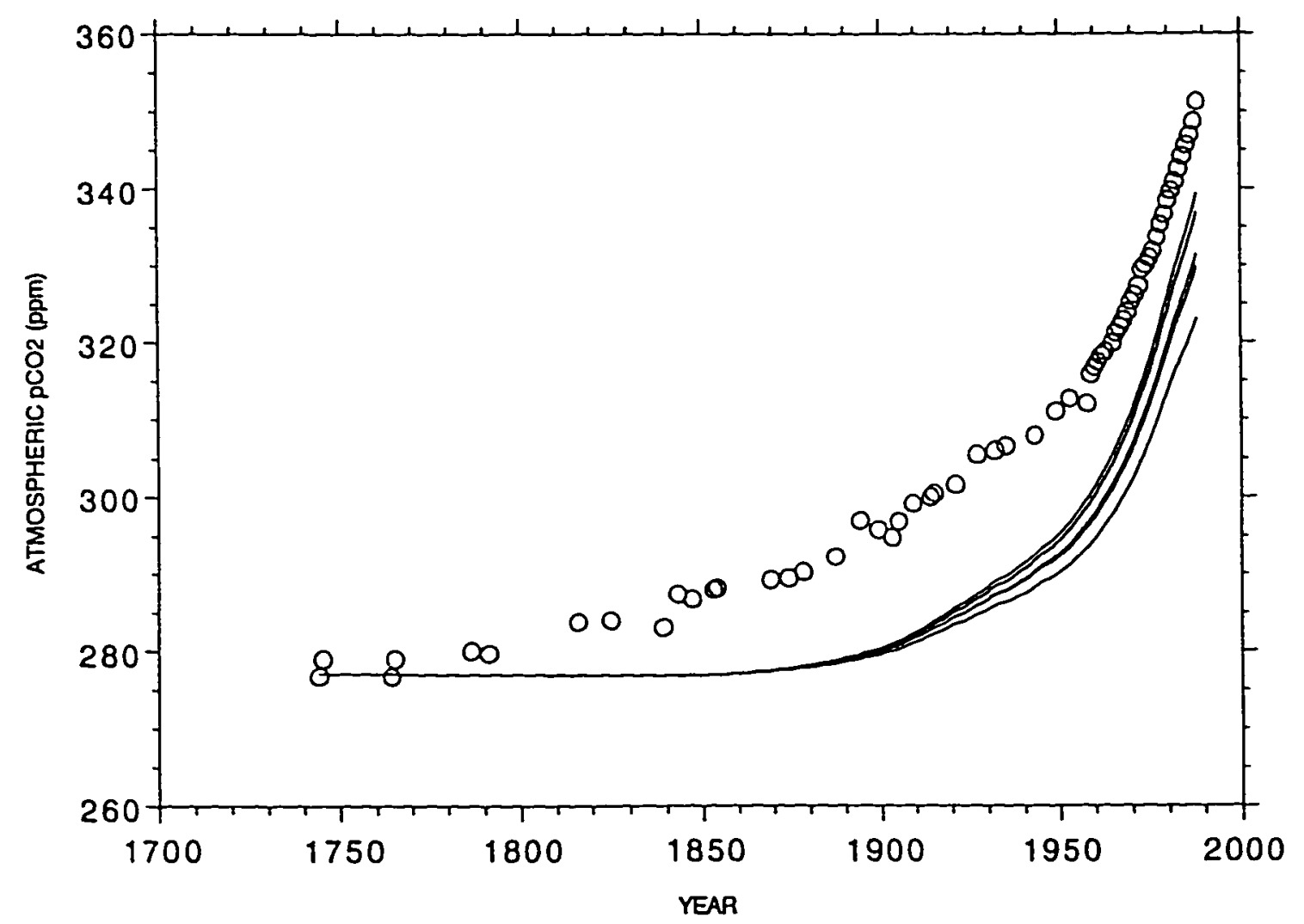

Figure 2.7 The responses of the ocean-atmosphere models to the historic fossil fuel forcing. All models were initialized at the 1744 ice core value for $\left[\mathrm{CO}_{2}\right]$. Open circles represent the atmospheric $\mathrm{CO}_{2}$ record (cf. Fig. 2.1). 


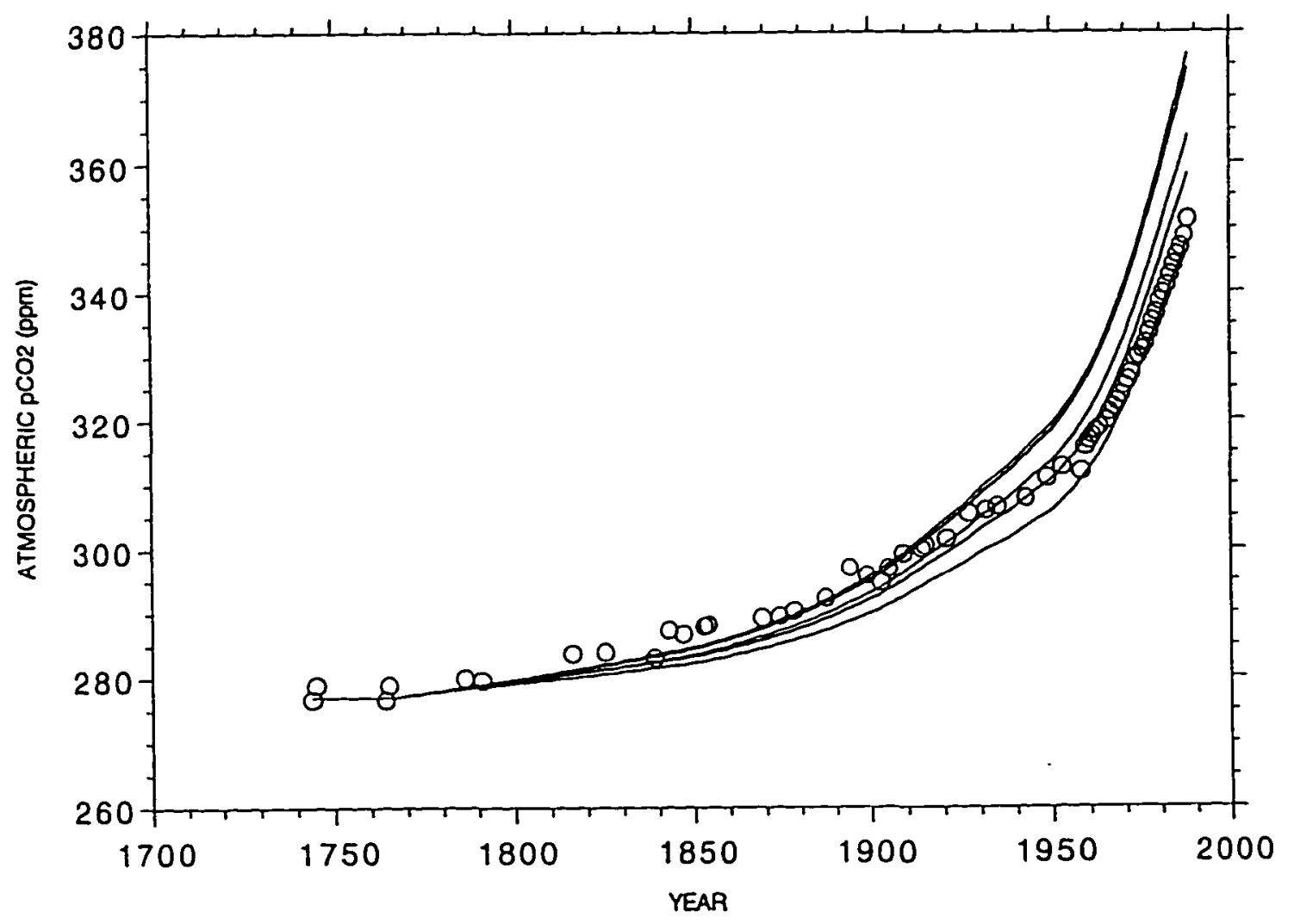

Figure 2.8 The responses of the ocean-atmosphere models to the historic fossil fuel forcing plus an estimate of biotic (deforestation) flux. All models were initialized at the 1744 ice core value for $\left[\mathrm{CO}_{2}\right]$. Open circles represent the atmospheric $\mathrm{CO}_{2}$ record (cf. Fig. 2.1). 


\section{The terrestrial carbon sub-model}

The net exchange of carbon between terrestrial vegetation and the atmosphere may be considered to be the sum of three fluxes: gross photosynthesis, autotrophic plant respiration, and heterotrophic (soil microbial) respiration (e.g., Aber and Melillo 1991). In addition, the anthropogenic perturbation of land conversion results in a net flux due to combustion minus regrowth. The gross fluxes between the terrestrial system and the atmosphere are similar to those for the oceans ( $100 \mathrm{Pg} \mathrm{C}$ per year; Fig. 2.3; see also Bolin et al. 1979, Bolin 1981), but the considerably smaller terrestrial pool size leads to a much faster turnover time than for the oceans (Björkstrom 1979).

Motivated by the issue of a hypothetical missing carbon sink and by plant physiological $\mathrm{CO}_{2}$ response, it has been suggested (e.g., Bacastow and Keeling 1973) that terrestrial vegetation may be "fertilized" by the increasing concentration of $\mathrm{CO}_{2}$. Though the issue is controversial (Strain and Cure 1985, Bazzaz 1990, Garbutt et al. 1990, Bazzaz and Fajer 1992. Diaz et al. 1993; see also Dai and Fung 1993 for a climate-based hypothesis and Houghton 1993a for a land-use-based hypothesis), it is possible that terrestrial ecosystems are responding to the rapid increase in atmospheric $\mathrm{CO}_{2}$ concentration by producing more biomass and/or storing more soil carbon, thereby balancing the global carbon budget (see Section 2.2 for a more detailed discussion). Consequently, there is the possibility that higher $\mathrm{CO}_{2}$ levels may lead to an increase in net primary production and perhaps net ecosystem production (carbon storage).

The possible existence of a fertilization effect, the rapid response of the land biota-soil system, and the fact that one of the major fluxes to the atmosphere $\left(F_{B}\right)$ is actually due to alteration of the terrestrial biosphere make it appropriate to include terrestrial dynamics in our attempt to address the issue of the lifetime of excess atmospheric $\mathrm{CO}_{2}$. Emanuel et al. (1984) 
have developed a highly aggregated model of global carbon cycling which is similar in scope to the three simple ocean models presented in the previous section. This model (Fig. 2.9) is composed of a set of eight coupled differential equations (Table 2.2; terrestrial components) which govern the flow of carbon between reservoirs representing the atmosphere, the surface ocean, the deep ocean, nonwoody parts of trees, woody parts of trees, ground vegetation, detritus/decomposers, and soils. We have replaced Emanuel's two-box ocean (Fig. 2.9) with the other simple ocean models that we are considering. In the current context, Emanuel's model is of interest, in part, because it is a convenient way to handle terrestrial carbon fluxes associated with forest clearing and re-establishment. The model deals with these activities in the following way:

1. A specified mass of carbon is released (due to land-use change) from "woody" and "nonwoody" parts of trees as a function of time, in a ratio determined by their relative sizes.

2. A fraction of the carbon released is transferred immediately to the atmosphere, and a fraction enters the detritus/decomposers pool. The remainder is assumed to be in a very slowturnover pool (e.g., timber products).

3. All vegetation pools experience a logistic-growth recovery with a prescribed rate, and with a dynamically varying asymptote.

4. Upon clearing, the asymptotic level for "trees" decreases, and the asymptotic level for "ground vegetation" increases.

We remark that given a clearing and abandonment time series, the interplay of these dynamics determines the flux $F_{B}$. We also note that the structure of the model allows a simple modification to include a fertilization effect, which we later discuss in detail.

Returning to the global carbon cycle, our basic approach is to modify the Emanuel global carbon cycle in two ways. First, we replace the two box ocean model by (in turn) the 
Table 2.2 The differential equations governing the terrestrial carbon dynamics in the Emanuel et al. (1984) model.

\begin{tabular}{ll}
\hline Compartment & Equation \\
\hline Atmosphere & $d C_{1} / d t=F_{F E}+\phi_{A} F_{B}+F_{O A}+\alpha_{51} C_{5}+\alpha_{61} C_{6}-\left(F_{12}+F_{13}+F_{14}\right)$ \\
Trees-nonwoody & $d C_{2} / d t=F_{12}-\alpha_{25} C_{2}-F_{B} C_{2} /\left(C_{2}+C_{3}\right)$ \\
Trees-woody & $d C_{3} / d t=F_{13}-\left(\alpha_{35}+\alpha_{36}\right) C_{3}-F_{B} C_{3} /\left(C_{2}+C_{3}\right)$ \\
Ground vegetation & $d C_{4} / d t=F_{14}-\left(\alpha_{45}+\alpha_{46}\right) C_{4}$ \\
Detritus/decomposers & $d C_{5} / d t=\alpha_{25} C_{2}+\alpha_{35} C_{3}+\alpha_{45} C_{4}-\left(\alpha_{56}+\alpha_{51}\right) C_{5}+\phi_{D} F_{B}+\psi F_{B}$ \\
Soil carbon & $d C_{6} / d t=\alpha_{36} C_{3}+\alpha_{46} C_{4}+\alpha_{56} C_{5}-\alpha_{61} C_{6}-\psi F_{B}$ \\
Assimilation fluxes: & \\
Trees-nonwoody & $F_{12}=v_{T} C_{2}-\rho_{T} C_{2}^{2}$ \\
Trees-woody & $F_{13}=\left(F_{13}{ }^{0} / F_{12}{ }^{0}\right) F_{12}$ \\
Ground vegetation & $F_{14}=v_{T} C_{4}-\rho_{V} C_{4}^{2}$ \\
\hline Variable & Definition \\
\hline$C_{i}$ & mass of carbon \\
$F_{i j}$ & flux from $i$ to j \\
$\alpha_{i j}$ & transfer coefficient from $i$ to $j$ \\
$F_{F E}$ & fossil CO ${ }_{2}$ flux \\
$F_{B}$ & land-use change CO \\
$F_{O A}$ & net ocean-atmosphere flux \\
$\phi_{A}$ & fraction of $F_{B}$ diverted to atmosphere \\
$\phi_{D}$ & fraction of $F_{B}$ diverted to detritus/decomposers \\
$\Psi F_{B}$ & soil to detritus flux associated with $F_{B}$ \\
$F_{i j}^{0}$ & post-disturbance equilibrium for $C_{2}$ \\
$v_{T} / \rho_{T}$ & \\
$v_{v} / \rho_{V}$ & \\
\hline &
\end{tabular}

box-diffusion model, the outcrop-diffusion model, and by the 12-box ocean model; and second, we develop a simple treatment of the "fertilization" term. By comparing the resulting hybrid models (E-BD, E-OC, and E-12B; both with and without fertilization) with results of the oceanatmosphere models (BD, OC, and $12 \mathrm{~B})$, we effectively perform the experiments on the 


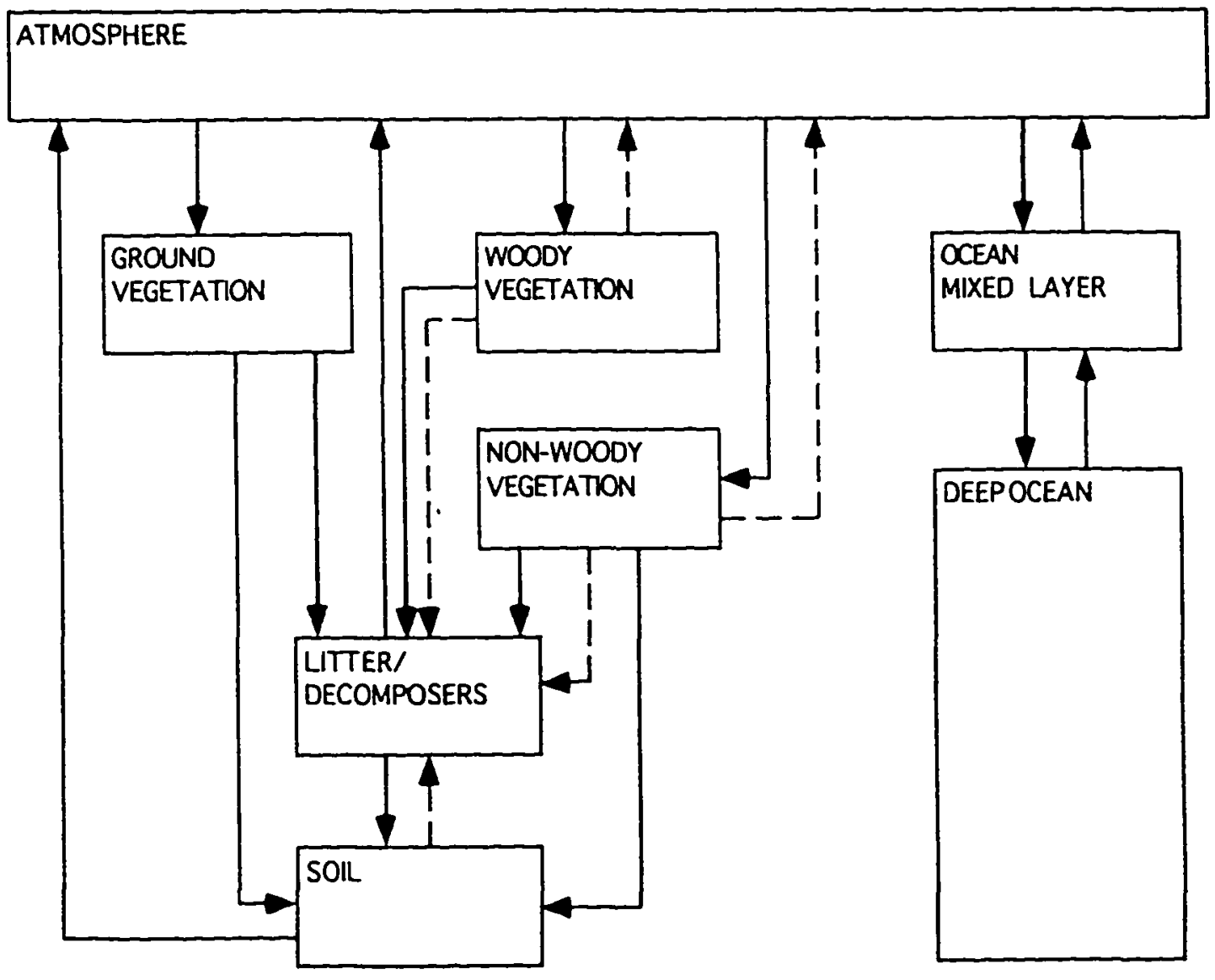

Figure 2.9 Globally averaged carbon cycle model (Emanuel et al. 1984). The atmosphere exchanges carbon with terrestrial components, and with the oceans. Solid arrows represent equilibrium (natural) carbon fluxes; dashed arrows represent fluxes associated with forest clearing activities. 
residence time of $\mathrm{CO}_{2}$ in the atmosphere with the terrestrial biosphere switched on and off. We are thus able to investigate the effects of a fertilization assumption plus forest regrowth or just forest regrowth on the residence time of $\mathrm{CO}_{2}$ in the atmosphere. The modeled forest regrowth develops from a deforestation pattern for the period 1700 to the present, and is constrained to produce the net flux in Fig. 2.2.

\subsubsection{Characterization of the biotic response}

As discussed in Section 2.2, if the ocean-atmosphere models are forced with $F_{F E}$ and $\mathrm{F}_{\mathrm{B}}$ as input and the results are compared to $\left[\mathrm{CO}_{2}\right]$, there is a discrepancy (i.e. an overshoot; see Fig. 2.8). This residual $R$ (the difference between the model output, $\left[\mathrm{CO}_{2}\right]^{M}$ and the $\left[\mathrm{CO}_{2}\right]$ data) can be decomposed as:

$$
R=\left(\left[\mathrm{CO}_{2}\right]-\left[\mathrm{CO}_{2}\right]^{M}\right)=R_{B}+R_{E 1}+R_{E 2}+R_{E 3}
$$

where $R_{B}$ is caused by some unspecified, hypothetical biological mechanism (e.g., the "fertilization factor"), $R_{E 1}$ results from inaccuracies in the models, $R_{E 2}$ results from uncertainty in the $\left[\mathrm{CO}_{2}\right]$ data, and $R_{E 3}$ is due to uncertainty in the $F_{F E}$ and $F_{B}$ data. We will not attempt to characterize the latter three, so let $R_{E}=R_{E 1}+R_{E 2}+R_{E 3}$. The expression then becomes:

$$
R=R_{B}+R_{E}
$$

Thus the residual has been reduced to two terms: error associated with some ignored physiological or ecological process and error associated with uncertainties in information (data or model). Further, we will make the following two assumptions: $R_{E} \ll R_{B}$, and the residual $R_{B}$ 
may be associated with an increment $F_{r}$ in the net flux of carbon from the atmosphere to the terrestrial biota:

$$
F_{r}=\rho_{r} \cdot F_{n p p}
$$

where (cf., Table 2.2),

$$
F_{n p p}=F_{12}+F_{13}+F_{14}
$$

To parameterize the fertilization factor $\rho_{r}$, there are several functional forms suggested in the literature, and by other natural systems. We have selected three different empirical relationships (logarithmic, logistic, and linear) to describe the fertilization enhancement as a function of elevated $\mathrm{CO}_{2}$ :

$$
\begin{aligned}
& \rho_{r}=a \cdot \log \left(C / C_{i}\right), \\
& \rho_{r}=a \cdot \tanh \left(b\left(C / C_{i}+c\right)\right), \\
& \rho_{r}=a \cdot\left(C / C_{i}\right)+b,
\end{aligned}
$$

where $C / C_{i}$ is the ratio of present to initial $\mathrm{CO}_{2}$, and $a, b$, and $c$ are free parameters. Note that this treatment will not only vary the functional dependence, but also the number of free parameters. To obtain the values for those parameters, we must compute $F_{r}$ and $F_{n p p}$ from the 
carbon models (E-12B, E-OC, and E-BD) and perform a least-squares fit of $\rho_{r}$ to $F_{r} / F_{n p p}$ (see Equation 2.9).

In order to compute the missing flux $F_{r}$, we specify that the rate of change of the atmospheric $\left[\mathrm{CO}_{2}\right]$ that is produced by the models is equivalent to the measured rate of change (i.e. $R=0$ ), and is given by:

$$
\frac{d C}{d t}=\frac{d C_{1}}{d t}+F_{r}
$$

where $d C_{1} / d t$ is the net flux to the atmosphere calculated by the models. and $d C / d t$ may be obtained from the $\left[\mathrm{CO}_{2}\right]$ record. Thus the residua! flux, for each time step, may be calculated by a deconvolution procedure:

$$
\begin{aligned}
& F_{r}(t)=\frac{d C}{d t}-\frac{d C_{1}}{d t} \\
& =\frac{d C}{d t}-F_{F E}+\phi_{A} F_{B}+F_{O A}+\alpha_{51} C_{5}+\alpha_{61} C_{6}-\left(F_{12}+F_{13}+F_{14}\right) .
\end{aligned}
$$

where we have substituted the terms from Table 2.2 on the right hand side. The method is straightforward: we add the flux $F_{r}$ to the right hand side of the derivative $(2.15)$ as the model proceeds. This is similar to the way in which deconvolution has been used to determine the biotic flux $F_{B}$ (e.g., Siegenthaler and Oeschger 1987), given the fossil flux, $F_{F E}$. Figure 2.10 shows the deconvolved missing flux $F_{r}$ for the ocean-atmosphere models and Fig. 2.11 shows the graph of $F_{r} / F_{n p p}$ vs. $C / C_{i}$ with the fitted curve for the logistic parameterization of $\rho_{r}$ using 
the E-12B model. We now have the following tools needed for our investigation: three oceanatmosphere models, the corresponding ocean-terrestrial-atmosphere models, an estimate of the evolution of the terrestrial component as a result of land-use, and a set of parameterizations for dealing with a hypothetical biotic response to increasing $\left[\mathrm{CO}_{2}\right]$.

\subsubsection{Single Half-Life}

Before investigating the response of these simple coupled carbon cycle models, we clarify our concerns about calculating atmospheric residence time of $\mathrm{CO}_{2}$ and suggest a simple index for model comparisons. The e-folding time characterizes the time it takes for a decaying exponential curve will fall to $1 / \mathrm{e}$ of its original value. The conventional determination of lifetime $\left(T_{\text {res }}\right)$ is conceptually not much more substantive, and its calculation may present a number of difficulties which reduce its utility. Thus we introduce a simple indicator of atmospheric residence time, the Single Half-Life $\left(T_{\mathrm{t} / 2}\right)$, which is the time required for the model atmosphere to relax from its present value (or some future value, though this would alter $T_{1 / 2}$ ) to one half of its new equilibrium value. This, of course, implies that we will not deal with the pulse response of the models, but with a more scenario-based approach: allow the models to respond to the historical inputs (e.g., $F_{F E}$ and $F_{B}$; see Fig. 2.2) and then cut the forcing to zero. From any cutoff time (in the past, present, or future) onward, we observe the decay of the concentration $c(t)$ of atmospheric $\mathrm{CO}_{2}$ towards its new equilibrium. We acknowledge that this concept or index focuses aftention upon the initial response and ignores the mid-term to very long term aspects of $c(t)$.

There are only two assumptions involved in these computations which are necessary to compare results across models: first, the models are all required to reproduce the historic $\left[\mathrm{CO}_{2}\right]$ record, and second, though not essential, the equilibrium value is approximately the same for 


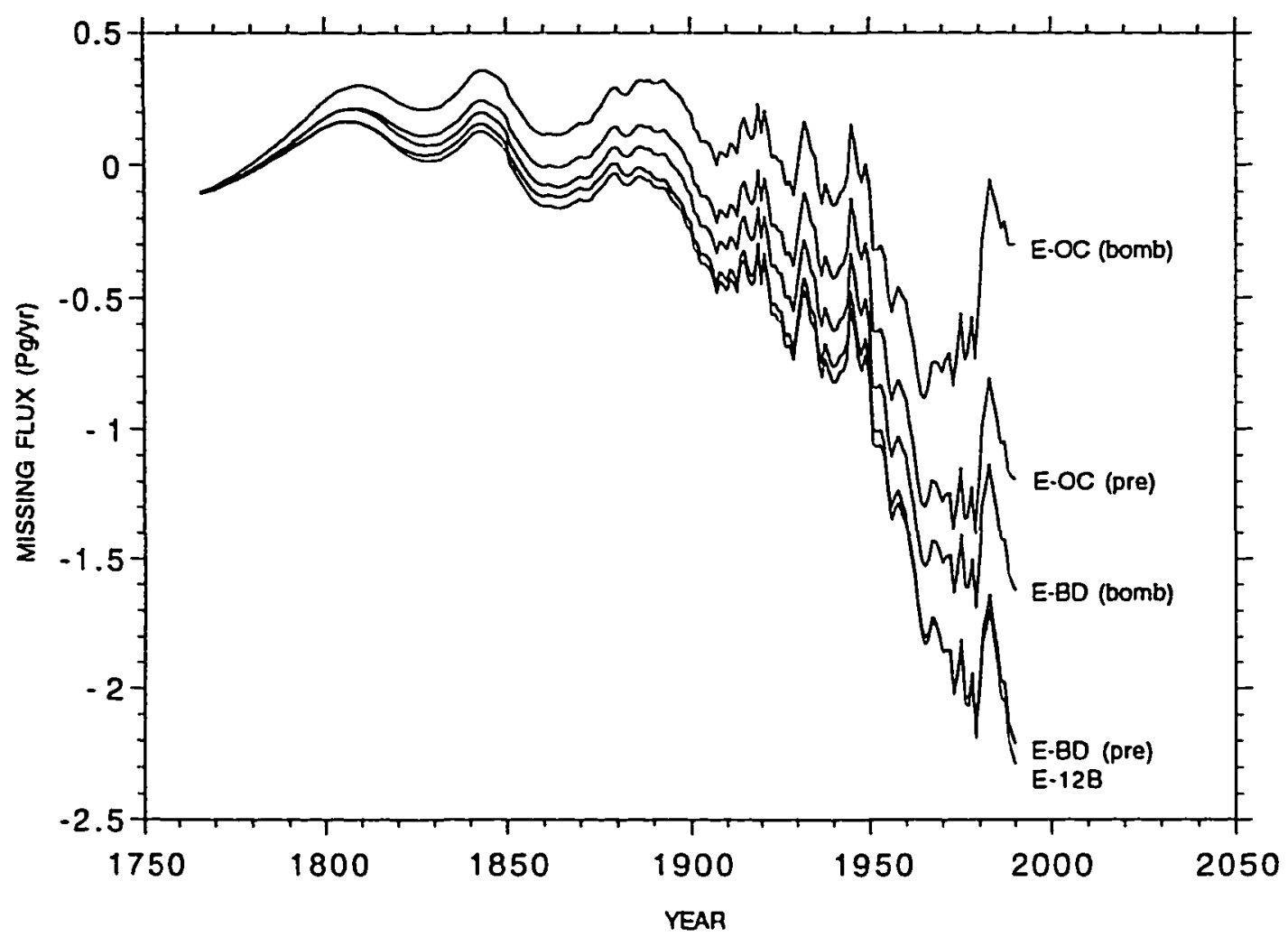

Figure 2.10 Deconvolved flux $\left(F_{r}\right)$ determined by the ocean-terrestrial-atmosphere models. This flux (generally a sink for later years) represents the additional input required for the models to reproduce the $\left[\mathrm{CO}_{2}\right]$ record; it may be associated with the hypothetical response of the terrestrial biosphere to increasing ambient $\mathrm{CO}_{2}$ concentrations. 


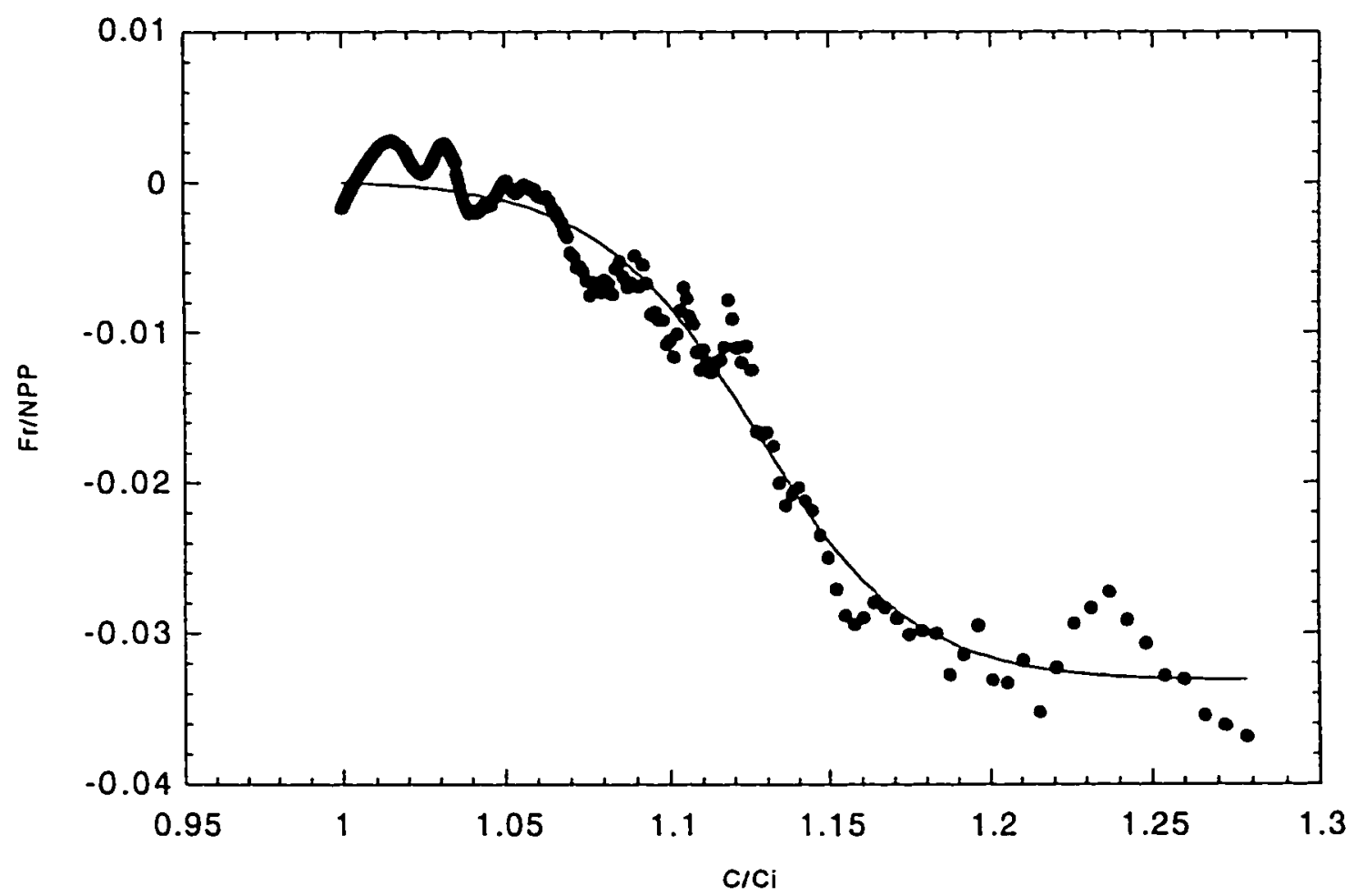

Figure 2.11 A fit of the function $\rho_{r}$ to the deconvolved flux divided by the net primary production flux as a function of relative atmospheric $\mathrm{CO}_{2}$ concentration. This graph shows the logistic parameterization (Equation 2.12). 
all models. This assumed equilibrium $\left[\mathrm{CO}_{2}\right]$ is a theoretical value based on the partitioning between atmosphere and ocean. Because of the carbonate buffer system, the relationship between a percentage increase in atmospheric carbon dioxide $\left(\Delta C_{a} / C_{a}\right)$ and a percentage increase in oceanic dissolved inorganic carbon $\left(\Delta C_{\mathrm{o}} / C_{\mathrm{o}}\right)$ may be approximated by:

$$
\Delta C_{a} / C_{a} \approx 10 \cdot\left(\Delta C_{\mathrm{o}} / C_{\mathrm{o}}\right)
$$

This factor of ten is called the Revelle factor. If we note that the cumulative release of $\mathrm{CO}_{2}$ from $F_{F E}$ and $F_{B}$ combined is equal to $377 \mathrm{Pg} \mathrm{C}$, and assume that the initial values for the total ocean DIC and atmospheric $\mathrm{C}$ are $38200 \mathrm{Pg}$ and $597 \mathrm{Pg}$ respectively, (2.16) yields an equilibrium partial pressure of $326 \mathrm{ppm}$. This number, which we use simply as a reference point, is the theoretical asymptote for the atmospheric $\mathrm{CO}_{2}$ decay curves $c_{i}(t)$. If we terminate the forcing at a present value (e.g., $t_{0}=354 \mathrm{ppm}$ ), $T_{\mathrm{l} / 2}$ is defined to be the first year after cutoff at which $c(t) \leq 326 \mathrm{ppm}$. We note that small differences could be obtained by varying the Revelle value, by using the more complete mathematical description of the carbonate-borate chemical system (e.g., Bolin et al. 1979. Bolin 1981), or by assuming different concentrations of dissolved inorganic carbon.

\subsubsection{Results using the Single Half-Life}

In this section, we explore the response of the global carbon cycle models (E-BD, E$\mathrm{OC}$, and $\mathrm{E}-12 \mathrm{~B})$ when the inputs $\left(F_{F E}\right.$ and $\left.F_{B}\right)$ are reduced instantaneously to zero under two separate conditions: when we allow $F_{r}$ to operate for all $t$, and when there is no $F_{r}$. We compare these results to a similar calculation of Single Half-Lives involving only the three ocean-atmosphere systems (BD, OC, 12B). Finally, to connect to the results reported by the 
IPCC (Watson et al. 1990), we compute in a subsequent section the classical lifetimes of $\mathrm{CO}_{2}$ using the three ocean-atmosphere models as well as when the terrestrial sink is included.

Tables 2.3 and 2.4 show the $T_{1 / 2}$ values calculated using the atmosphere-ocean models (12B, BD, and $\mathrm{OC}$ ) standing alone (Table 2.3), using the coupled terrestrial models (E-12B, E$\mathrm{BD}$, and $\mathrm{E}-\mathrm{OC}$; the latter two, again, with two parameterizations for the ocean submodels) with regrowth alone assumed (Table 2.4, row 1), and assuming both regrowth and fertilization. Fertilization is described with the three different schemes discussed in Section 2.3 (Table 2.4, rows $2-4$ ). We can see that without the fertilization flux, the models yield Single Half-Lives from as high as 116 years (BD, preindustrial ${ }^{14} \mathrm{C}$ calibration) to as low as 37 years $(\mathrm{OC}$, bomb ${ }^{14} \mathrm{C}$ calibration); the ranges narrow considerably if the Outcrop-Diffusion (OC) model is dropped. It also narrows when the biosphere is included with regrowth alone assumed. This simply adds a uniform sink to each system, which aids the ocean-atmosphere systems that have an ocean surface "bottleneck" problem in the removal of carbon (i.e. the OC and 12B systems). Thus, the Single Half-Lives are obviously reduced; they are even more significantly reduced if one includes a fertilization sink (Table 2.4 , rows $2-4$ ). In part, this is simply the addition of a sink, as was the case in including forest regrowth; however, there is a further compensating factor that the sink strength is not uniform. Namely, the model oceans which are more efficient at taking up excess $\mathrm{CO}_{2}$ require a smaller $F$, flux to match the $\left[\mathrm{CO}_{2}\right]$ record, and vice-versa. This is also reflected in the regression coefficients for the OC models (see Table 2.5); $F_{r}$ is close enough to zero that random fluctuations in the residual nearly overwhelm the assumed logarithmic trend.

Plots of the relaxation curves associated with the ocean-atmosphere results (Table 2.3), for all ocean models, are shown as Fig. 2.12. This should be compared to the somewhat steeper initial drop when the terrestrial biosphere is included with regrowth alone and the even 
Table 2.3 Single Half-Life results for the ocean-atmosphere models.

\begin{tabular}{lc}
\hline Ocean Model $^{*}$ & $T_{1 / 2}(\mathrm{yr})$ \\
\hline 12-Box model (12B) & 81 \\
Box-Diffusion (BD) ${ }^{1}$ & 116 \\
Outcrop-Diffusion (OC) ${ }^{1}$ & 42 \\
Box-Diffusion (BD) ${ }^{2}$ & 79 \\
Outcrop-Diffusion (OC) & 37 \\
\hline${ }^{1}$ Calibrated with pre-industrial ${ }^{14} \mathrm{C}$. \\
${ }^{2}$ Calibrated with bomb-produced ${ }^{14} \mathrm{C}$.
\end{tabular}

Table 2.4 Single Half-Life results (in years) for the ocean-atmosphere-terrestrial models under various $\mathrm{CO}_{2}$ fertilization assumptions.

\begin{tabular}{lccccc}
\hline Parameterization & E-12B & E-BD $^{1}$ & E-OC $^{1}$ & E-BD $^{2}$ & E-OC $^{2}$ \\
\hline None (regrowth only) & 68 & 86 & 37 & 63 & 33 \\
Log & 22 & 49 & 28 & 40 & 25 \\
Linear & 19 & 30 & 20 & 26 & 17 \\
Logistic & 20 & 38 & 23 & 33 & 20 \\
\hline
\end{tabular}

${ }^{1.2}$ As in Table 2.3.

${ }^{3}$ No fertilization flux in this experiment.

Table 2.5 Coefficient of correlation $(R)$ for the fit of $F_{r}$ to the calculated residual carbon flux.

\begin{tabular}{lccccc}
\hline Parameterization & E-12B & E-BD & E-OC & E-BD & E-OC $^{2}$ \\
\hline Log & .896 & .914 & .804 & .869 & .746 \\
Linear & .953 & .956 & .895 & .931 & .717 \\
Logistic & .986 & .986 & .958 & .975 & .400 \\
\hline
\end{tabular}

${ }^{1.2}$ As in Table 2.3

steeper slopes when fertilization is assumed (Fig. 2.13); in order to compare Figs. 2.12 and 2.13, we include in Fig. 2.13 the relaxation dynamics of the Box-Diffusion Model with the ${ }^{14} \mathrm{C}$ bomb data used for parameterization. Note that the functional differences for different schemes to incorporate fertilization are relatively unimportant; this is a result partly of the deconvolution 


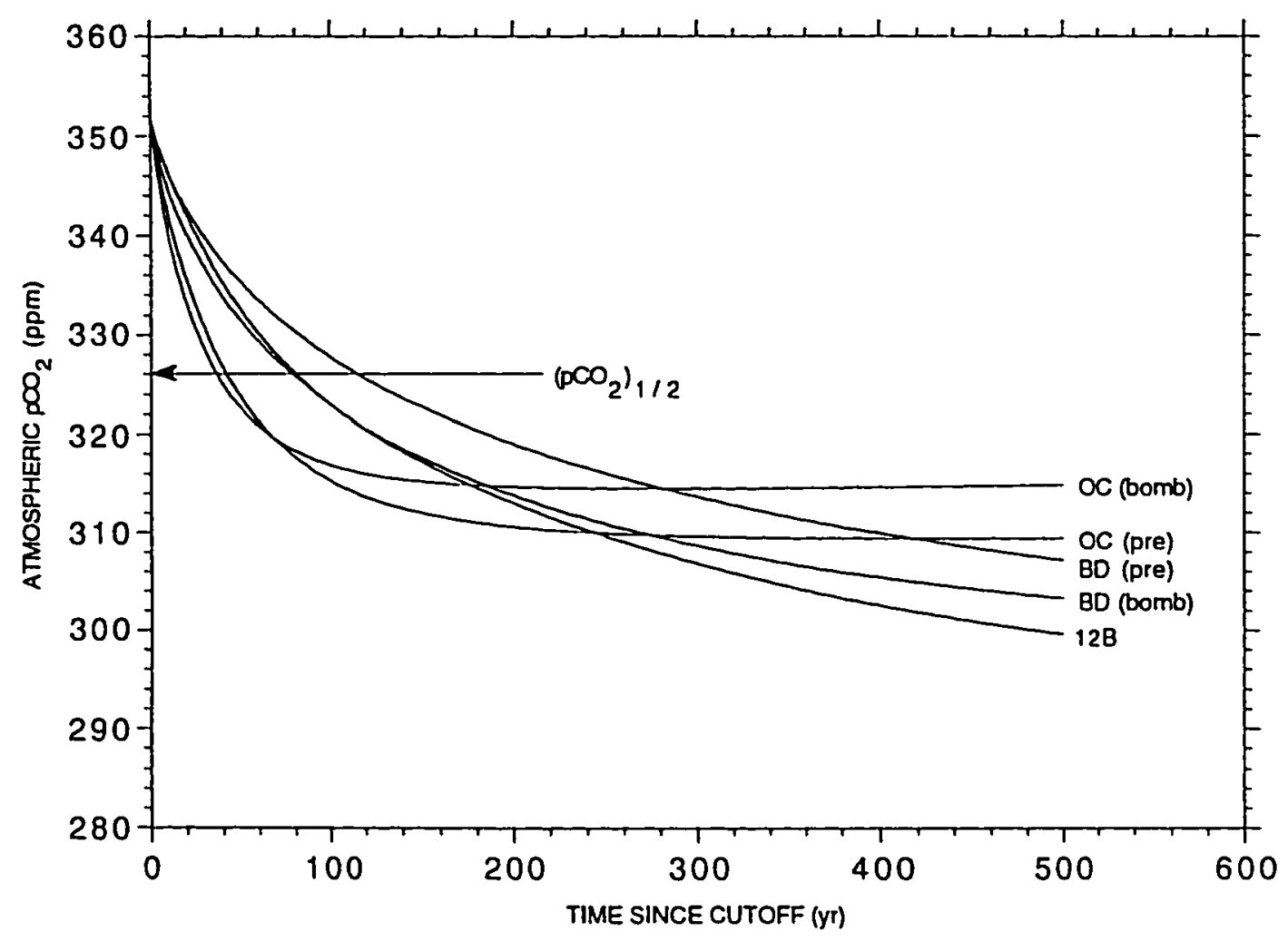

Figure 2.12 Response of the ocean-atmosphere models to a cessation of all emissions at $t_{0}=1990$. The horizontal arrow represents the $\left[\mathrm{CO}_{2}\right]$ level halfway between the 1990 value and the theoretical equilibrium value. 


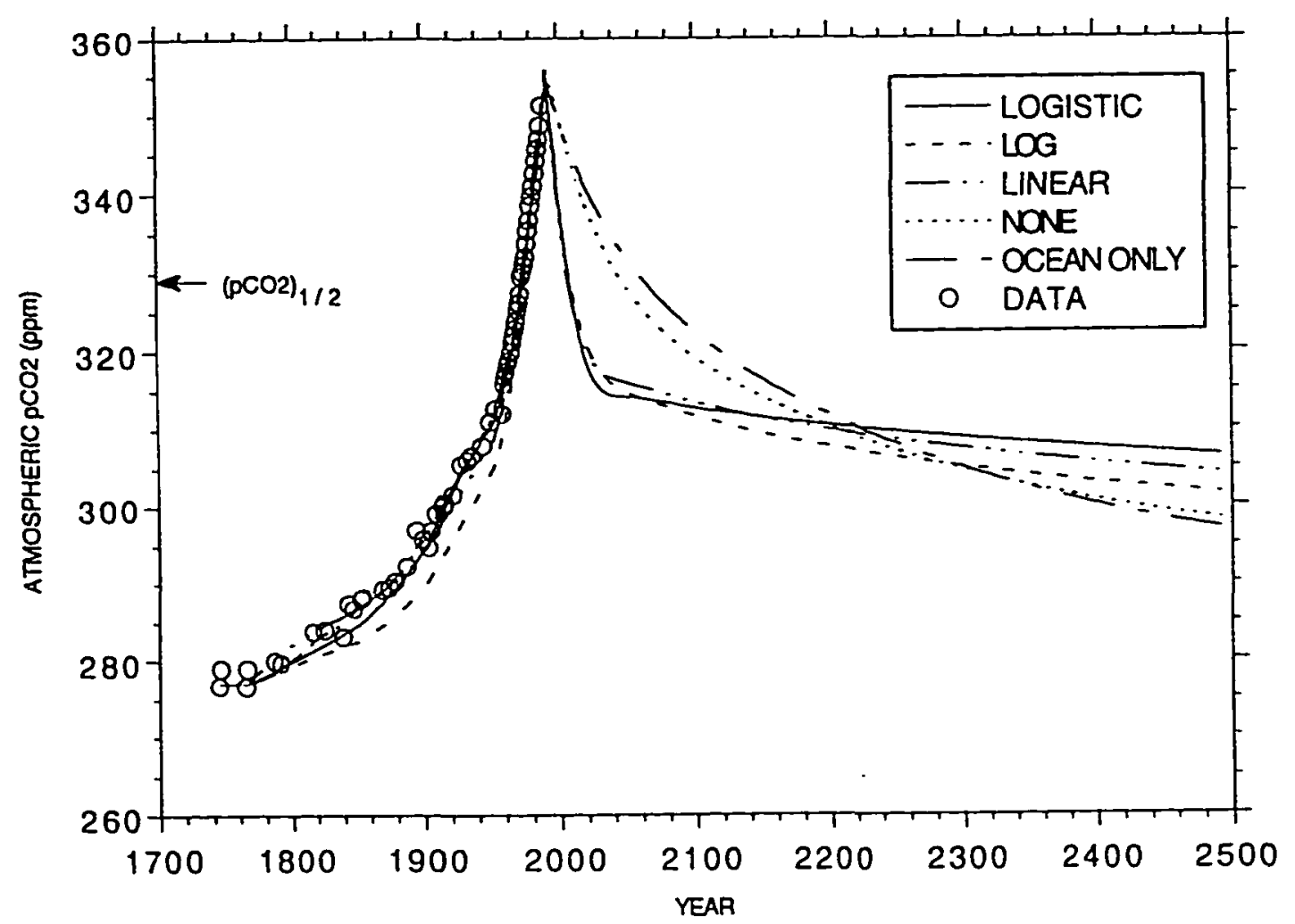

Figure 2.13 Response of the E-12B (Emanuel terrestrial submodel plus twelve-box ocean) model to the emissions cutoff scenario. Curves are shown which represent the inclusion of three possible parameterizations of fertilization flux, as well as the curve for no fertilization. For comparison we also include the curve for the ocean-only (12B) case. 
methodology which forces the dynamics to fit the same pattern, namely the ice core record.

In summary, there are small differences in Single Half-Life results associated with the model ocean used and with the nature of the parameterization of the missing flux $F_{r}$, but the principal variation is due predominantly to the inclusion of a terrestrial carbon sink, beyond forest regrowth. Thus, the results may be grouped into two categories (with associated average values): (1) regrowth and fertilization ( $\overline{T_{1 / 2}}=27$ years), (2) regrowth and no fertilization $\left(\overline{T_{1 / 2}}=57\right.$ years), and (3) no biosphere $\left(\overline{T_{1 / 2}}=71\right.$ years). If we drop the OC model, which (as mentioned earlier) mixes atmospheric $\mathrm{CO}_{2}$ instantaneously to deep waters and likely overestimates the rate of ocean uptake, and calculate averages using the $12 \mathrm{~B}$ model and two versions of the BD model, the Single Half-Lives increase somewhat as follows: (1) regrowth and fertilization $\left(\overline{T_{1 / 2}}=31\right.$ years), (2) regrowth and no fertilization $\left(\overline{T_{1 / 2}}=72\right.$ years), and (3) no biosphere ( $\overline{T_{1 / 2}}=92$ years). The question of short-term atmospheric $\mathrm{CO}_{2}$ retention is effectively bracketed by these values.

\subsubsection{Exponential characterizations of lifetime}

We now turn to a discussion of the traditional notion of atmospheric lifetime and point out some of the difficulties that are inherent in this method when applied to a response which is not an exponential decay. For this discussion, we use the definition of Lashof and Ahuja (1990). The underlying idea in their definition of atmospheric lifetime is the classical concept of exponential decay:

$$
c(t)=\exp (-t / \tau),
$$


where $\tau$ is the decay constant (e-folding) time for the material. There is an immediate consequence of using this simple expression. Namely, the concentration $c(t)$ goes rapidly to zero as $t$ goes to infinity and the integral in Equation 2.17 is $\tau$. To use this basic concept of exponential decay, Lashof and Ahuja (1990) (see also Maier-Reimer and Hasselmann 1987) must accommodate two facts about (most) models of atmospheric $\mathrm{CO}_{2}$. Given a unit pulse of carbon dioxide, the decay is neither exponential nor does it go to zero. Their accommodation is to fit the response to a weighted sum of exponential decay functions and fix a finite time window for the fit, which in effect ignores the atmospheric concentration after a given length of time.

The mathematical construct requires the following: the calculated concentration $c(t)$ of atmospheric $\mathrm{CO}_{2}$ following a pulse at time zero in a given atmosphere-ocean model; a temporal interval (the period of consideration, $\left[0, N_{w}\right]$ ): an upper bound $\tau_{\text {mar }}$ on the time constant; and the number of terms $N$ to be used in the approximation process (the number is not important, only the issue of goodness-of-fit really matters). One then finds, by constrained least squares fit, non-negative numbers (weights) $a_{1}, \ldots, a_{N}$, where $a_{1}+\ldots+a_{N}=1$, and numbers (e-folding times) $\tau_{1}, \ldots, \tau_{N}$ such that

$$
c(t) \approx \Sigma_{i} a_{i} \cdot \exp \left(-t / \tau_{i}\right) ; \quad\left(0 \leq t \leq N_{\mathrm{w}} \text { and } 0 \leq \tau_{i} \leq \tau_{\text {max }}\right)
$$

By integration of (2.18) the atmospheric lifetime, $T_{\text {res }}$, is then simply the weighted average of the $\tau$ 's (an average e-folding time), i.e.,

$$
T_{\text {res }}=\Sigma_{i} a_{i} \cdot \tau_{i}
$$


Lashof and Ahuja (1990) used Maier-Reimer and Hasselman's (1987) results and presented a $\mathrm{CO}_{2}$ residence time value of 230 years.

Using the three atmosphere-ocean submodels, we inject an amount of $\mathrm{CO}_{2}$ into the atmosphere component of the models equal to $25 \%$ of the amount presently in our atmosphere. Each system then relaxes to different steady-state values (Fig. 2.14), with approximately 10$20 \%$ of the pulse remaining, depending on the specification of equilibrium carbonate chemistry in the model. This relaxation curve is approximated by a weighted sum of exponentials (i.e., Equation 2.18) under assumptions of $N_{w}, N$, and $\tau_{\max }$. The results are summarized in Table 2.6. Some of the results are, at first glance, counter-intuitive; the most efficient ocean model (OC) can yield a longer calculated atmospheric lifetime than the less efficient ocean models. This is an artifact of the relative quickness that the OC-model reaches an asymptotic value. Simply stated, the greater the overlap of the fitting window $\left(0 \leq t \leq N_{w}\right)$ with a period where the change (or the derivative) in the simulated atmospheric concentration is near zero, the greater the necessity that the fitting term must contain exponentials that are as flat as possible (i.e., very large $\tau$ 's).

For the purposes of comparison, we used $N_{w}$ values of 50,100,200,500, and 1000 years (Table 2.6). In general, increasing the interval (for a given model, and leaving $\tau_{\max }$ fixed) leads to an increase in the value of $T_{\text {res }}$ or a failure of the fitting routine since the larger interval includes more of the curve that is relatively flat. This sensitivity is why the choice of a reasonable value for $N_{w}$ is important; unfortunately there is no clear guideline; moreover, this sensitivity to $N_{w}$ is also connected with the sensitivity of $T_{\text {res }}$ to the assumed $\tau_{\max }$.

To the extent that $c(t)$ approaches 0 for $t \rightarrow N_{w}$, then the least-squares routine will attempt to return very high $\tau_{i}$ values (because the function is not decaying rapidly). Consequently, one needs to place a size constraint on the $\tau$ 's (i.e. $\tau_{\max }$ ). Unfortunately, for a 


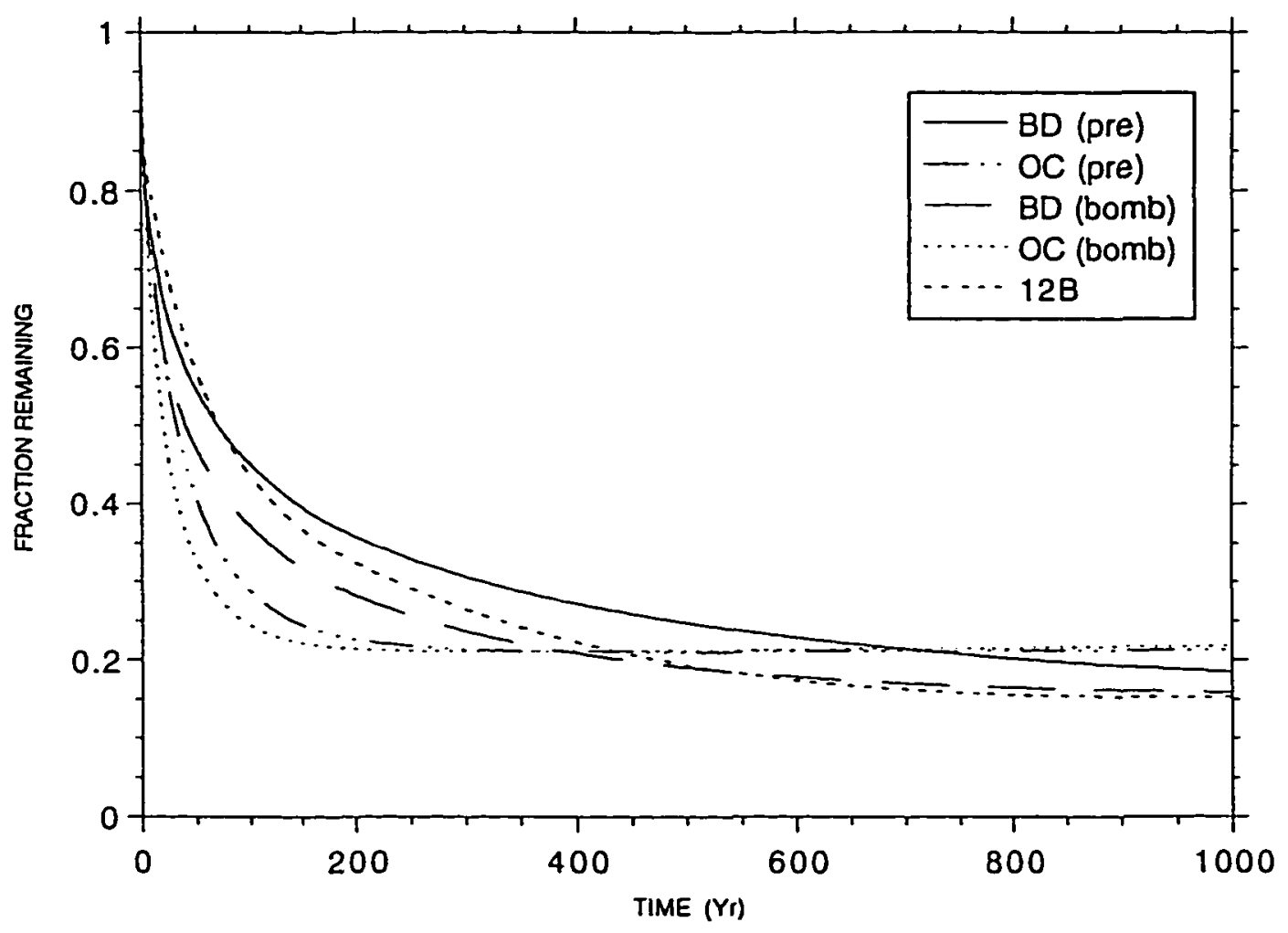

Figure 2.14 Response of the ocean-atmosphere models to an instantaneous pulse of $\mathrm{CO}_{2}$ equivalent to $25 \%$ of the present value. The origin of the time axis is $t=0$ because no historical emissions are used. 
Table 2.6 Sensitivity of $T_{\text {res }}$ for the ocean-atmosphere models.

\begin{tabular}{cc|ccc}
\hline \multicolumn{2}{c|}{ Numerical parameters } & \multicolumn{3}{c}{$T_{\text {res }}$ for each ocean model $(\mathrm{yr})^{1}$} \\
\hline$N_{w}(\mathrm{yr})$ & $\tau_{\max }(\mathrm{yr})$ & $\mathrm{BD}^{2}$ & $\mathrm{OC}^{2}$ & $12 \mathrm{~B}$ \\
\hline 50 & 500 & 132.7 & 114.3 & 236.8 \\
50 & 1000 & 262.1 & 202.9 & 231.6 \\
50 & 5000 & 1133.0 & 930.1 & 612.0 \\
100 & 500 & 170.9 & 123.0 & 243.4 \\
100 & 1000 & 253.8 & 205.4 & - \\
100 & 5000 & 667.5 & 871.0 & - \\
200 & 500 & 204.9 & 160.3 & 256.3 \\
200 & 1000 & 287.4 & 271.2 & 262.8 \\
200 & 5000 & 250.6 & 567.2 & 262.8 \\
500 & 500 & 230.1 & - & 259.9 \\
500 & 1000 & 320.7 & - & 348.8 \\
500 & 5000 & 490.5 & 1147.0 & 283.9 \\
1000 & 500 & - & - & - \\
1000 & 1000 & - & - & - \\
1000 & 5000 & 1015.0 & - & 963.7 \\
\hline
\end{tabular}

${ }^{1}$ Missing values indicate poor fit $\left(X^{2} \geq 0.1\right)$.

${ }^{2}$ Calibrated with bomb- ${ }^{14} \mathrm{C}$, as in Table 2.3

Table 2.7 Goodness of fit for selected runs from Table 2.6.

\begin{tabular}{cc|ccc}
\hline \multicolumn{2}{c|}{ Numerical parameters } & \multicolumn{4}{c}{$X^{2}$ of fit for each ocean model } \\
\hline$N_{\mathrm{w}}(\mathrm{yr})$ & $\tau_{\operatorname{mar}}(\mathrm{yr})$ & BD & OC & $12 \mathrm{~B}$ \\
\hline 50 & 500 & $1.9 \mathrm{e}-7$ & $1.2 \mathrm{e}-5$ & $7.1 \mathrm{e}-8$ \\
50 & 1000 & $1.2 \mathrm{e}-5$ & $1.1 \mathrm{e}-5$ & $3.8 \mathrm{e}-8$ \\
50 & 5000 & $1.1 \mathrm{e}-5$ & $9.7 \mathrm{e}-6$ & $3.8 \mathrm{e}-8$ \\
1000 & 500 & 3.92 & 12.9 & 1.34 \\
1000 & 1000 & 0.21 & 0.27 & 0.10 \\
1000 & 5000 & $1.1 \mathrm{e}-3$ & 0.20 & $1.4 \mathrm{e}-2$ \\
\hline
\end{tabular}

given window $N_{w}$, the calculated residence time is strongly dependent on this constraint (Table 2.6). This is partly a numerical artifact of the over-determined inversion process, in which 
minimizing error can lead to instabilities (see the $X^{2}$ values in Table 2.7). Figure 2.15 shows a plot of $c(t)$ for two different values of $\tau_{\max }$. The fitted curves are very different beyond the window $N_{w}$.

It appears that the numerical sensitivity of $T_{\text {res }}$ may not have been sufficiently well appreciated; however, Lashof and Ahuja (1990) do note this sensitivity to one aspect of the calculation when they discuss changing the constraint $\tau_{i} \leq 1000$. In their study, this $\tau_{\max }$ sensitivity was dealt with in a different way. They did not fit a $c(t)$ term, but rather used parameter values (based upon a Green's function) from a Maier-Reimer and Hasselman (1987) study: $\tau_{i}=(1000,362.9,73.6,17.3,1.9)$, and $a_{i}=(0.131,0.201,0.321,0.249,0.098)$. In other words, they took as given a weighted exponential form for $c(t)$. It was noted that the large $\tau_{0}=1000$ value effectively discounts long-term $\mathrm{CO}_{2}$ retention. This value was a fitting constraint used by Maier-Reimer and Hasselmann (1987). Specifically, changing $\tau_{0}$ from 1000 to 3000 and re-fitting the $a_{i}$ values led to a more than doubling of residence time from 230 to 500 years. This is consistent with our results.

In an attempt to connect these results with the earlier Single Half-Life calculations, we perform the same experiment with the scenario-based relaxation of the $\mathrm{BD}$ and the $\mathrm{E}-\mathrm{BD}$ models, with terrestrial biosphere and no fertilization, and with a $\mathrm{CO}_{2}$-fertilized terrestrial biosphere (Table 2.8). We find the same problems associated with the calculation of $T_{\text {res. }}$. Often the combinations that clearly reduce $\mathrm{CO}_{2}$ concentration the fastest, such as including fertilization, have longer lifetimes. There was a similar counter-intuitive result mentioned when discussing the results of the Atmosphere-Ocean systems (Table 2.6); the most efficient ocean model $(\mathrm{OC})$ often yielded a longer calculated atmospheric lifetime than the less efficient ocean models.

There are a number of difficulties with the exponential-based method that should be 


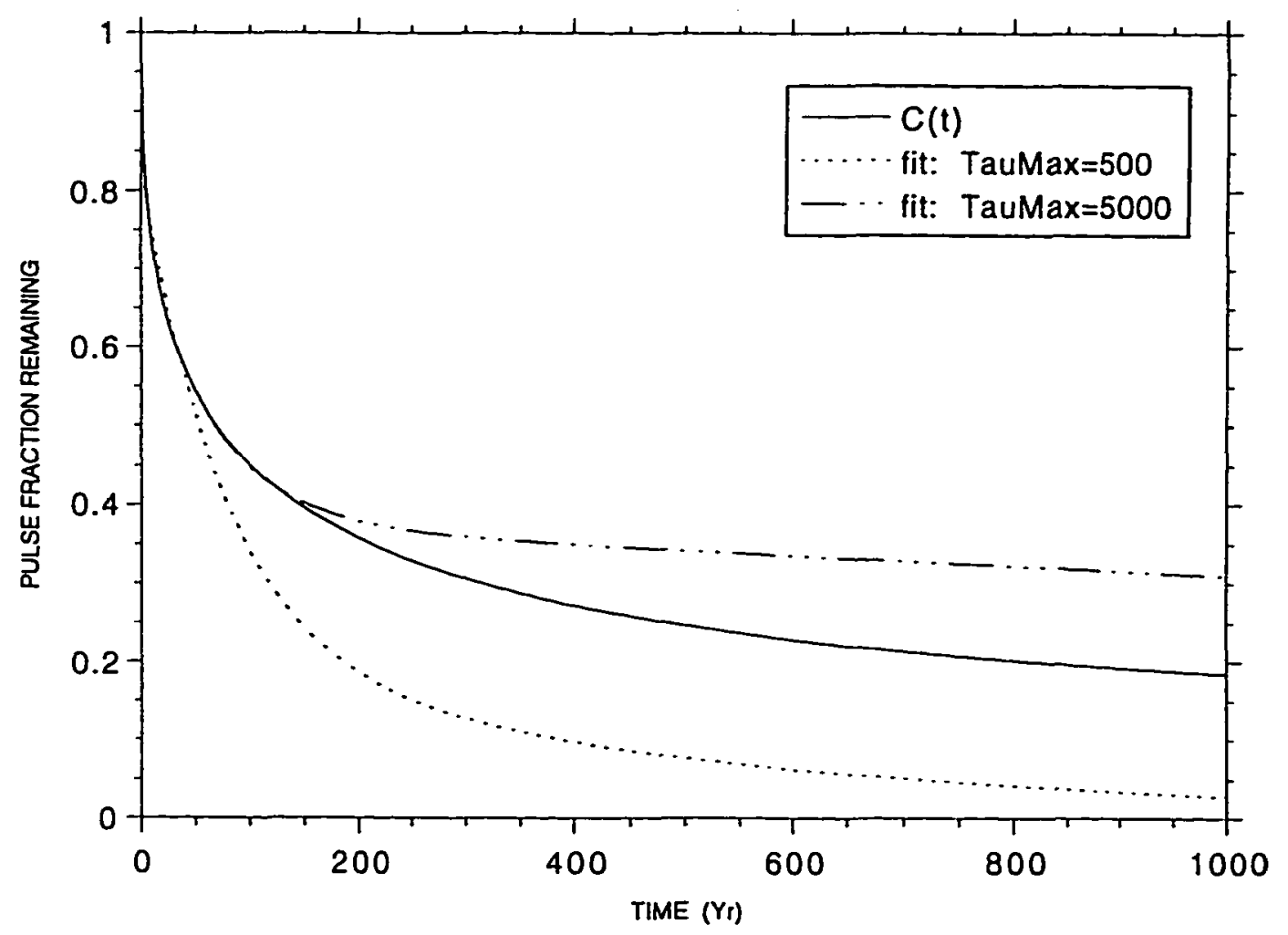

Figure 2.15 Response of the box-diffusion model to an instantaneous pulse, and two curves fitted by the least-squares method to the response $c(t)$. For these curves, $N_{w}=50, N=4$, and there are two different values of $\tau_{\max }(500$ and 5000). 
Table 2.8 Sensitivity of $T_{\text {res }}$ for the E-BD ocean-atmosphere-terrestrial model.

\begin{tabular}{|c|c|c|c|}
\hline \multicolumn{2}{|c|}{ Numerical parameters } & \multicolumn{2}{|c|}{$T_{\text {res }}$ for E-BD model $(\mathrm{yr})^{1}$} \\
\hline$N_{w}(\mathrm{yr})$ & $\tau_{\max }(\mathrm{yr})$ & Biosphere included & Fertilized biosphere \\
\hline 50 & 500 & 351.8 & 258.2 \\
\hline 50 & 1000 & 561.6 & 465.5 \\
\hline 50 & 5000 & 1591.6 & 2128.7 \\
\hline 100 & 500 & 375.4 & 273.7 \\
\hline 100 & 1000 & 391.9 & 512.6 \\
\hline 100 & 5000 & 401.1 & 2334.8 \\
\hline 200 & 500 & 379.9 & - \\
\hline 200 & 1000 & 560.3 & 528.2 \\
\hline 200 & 5000 & 1354.1 & 1186.7 \\
\hline 500 & 500 & - & - \\
\hline 500 & 1000 & 619.1 & - \\
\hline 500 & 5000 & 840.6 & 2135.3 \\
\hline
\end{tabular}

${ }^{1}$ Missing values indicate poor fit $\left(X^{2} \geq 0.1\right)$.

addressed. Based on these results, we may conclude that most of the $T_{\text {res }}$ values are, in some sense, determined by the constraints placed on the parameters $\tau_{i}$, and by the assumption about $N_{w}$. In other words, $T_{\text {res }}$ could be considered arbitrary unless there is some otherwise logical means for choosing (based on physical considerations) reasonable fitting constraints.

\section{$\underline{2.3 .7 \text { Summary }}$}

It is necessary for policy applications to have a consistent method for estimating the relative contributions of the anthropogenic, radiatively active trace gases to potential climate change. The greenhouse warming potential (GWP) is one method, but we have shown in Section 2.3.6 that the estimation of the integral in the denominator of the GWP formula (Equation 2.5) is ambiguous. As an interim step, we have introduced a simple indicator of the Earth system's ability to remove excess atmospheric $\mathrm{CO}_{2}$, the Single Half-Life $\left(T_{1 / 2}\right)$. This 
indicator is free from the numerous difficulties and numerical instabilities associated with a conventional lifetime calculation. Thus, one could envision a modified GWP, in which the Single Half-Lives of the radiatively important gases replace the integral in Equation 2.5.

We have found that the inclusion of an active (donor and recipient-controlled) terrestrial biosphere considerably alters modeled estimates of the effective lifetime of atmospheric $\mathrm{CO}_{2}$. Moreover, since this factor is the dominant source of variation, we group the results into three categories: biosphere with fertilization flux $\left(\widetilde{T_{1 / 2}}=27\right.$ years), and biosphere without fertilization flux $\left(\overline{T_{1 / 2}}=57\right.$ years). Finally, if there is no biosphere (purely an ocean atmosphere system; Table 2.3), then $\overline{T_{1 / 2}}=71$ years. If we drop the Outcrop-Diffusion Model, which is too efficient in taking up $\mathrm{CO}_{2}$ from the atmosphere, then these results change in detail but not in pattern: biosphere with fertilization flux $\left(\overline{T_{1 / 2}}=31\right.$ years $)$, and biosphere withour fertilization flux $\left(\overline{T_{1 / 2}}=72\right.$ years). Finally, if there is no biosphere (purely an ocean atmosphere system; Table 2.3 ), then $\overline{T_{1 / 2}}=92$ years.

The inclusion of an active (donor and recipient-controlled) terrestrial biosphere clearly and considerably alters our estimates of the effective lifetime of atmospheric $\mathrm{CO}_{2}$. Several important and difficult scientific questions remain. Does this terrestrial sink exist? Will it continue to operate or has it begun to saturate? What is the effect of changes in other biogeochemical cycles? What if climate begins to shift; does that increase or decrease the terrestrial sink? How will the oceans respond? These questions have generated, and continue to drive a great deal of research, and they are relevant to policy decisions surrounding understanding and mitigation of possible future global change. 


\subsection{Complement: Timescales of Atmospheric $\mathrm{CO}_{2}$ and Radiative Forcing Indices}

Following the publication of the above work, a comment was published in the same journal (Gaffin et al. 1995) in response. Their note sought to clarify some perceived confusion about the meaning of lifetime of excess atmospheric $\mathrm{CO}_{2}$, uncertainties in the carbon cycle itself, and implications for the calculation of greenhouse warming potentials (GWPs). Their comment consists of four main points, which we will review and respond to one by one in this section. Following this response, we will discuss how the Intergovernmental Panei on Climate Change approached the GWP issue (Albritton et al. 1993), effectively avoiding the problems associated with the fact that atmospheric $\mathrm{CO}_{2}$ is not well represented by a single lifetime. Finally, other characteristic measures of timescale will be presented that may give insight into the dynamics of carbon dioxide in the global atmosphere.

The first issue that Gaffin et al. (1995) raised was that "the present short term removal rate of atmospheric $\mathrm{CO}_{2}$ is strongly constrained simply by requiring models to fit the $\mathrm{CO}_{2}$ data." The short term removal rate they refer to is the average turnover time of excess $\mathrm{CO}_{2}$ which can be calculated by dividing the concentration $C$ by the input flux $F: \tau(t)=C(t) / F(t)$. Making this calculation using fossil emissions alone yields turnover times that typically range between 20-40 yr for the historic record (ca. 1960-1990), though there are a few high values $(50-70 \mathrm{yr})$. It is reasonable to assert that if a model is constrained to have the same inputs and outputs to the atmosphere as are measured, then the model atmosphere will have approximately the same $20-40 \mathrm{yr}$ turnover time. However, the assertion does not particularly bear upon our conclusions. We attempted to show how one particular measure of lifetime $\left(T_{1 / 2}\right)$ would be affected by different assumptions related to the inclusion and activity of the terrestrial biosphere. Moreover, our analysis could have focused upon turnover time $(\tau)$ itself as an indicator. Atmospheric $\mathrm{CO}_{2}$ turnover time is not a fixed property; indeed, it is multiply defined 
for a system that is not at steady-state (i.e., $F$ could refer to inputs or outputs), and it is sensitive to assumptions about global source/sink dynamics.

The second point raised is the idea that "a strong precedent exists from reservoir theory for defining lifetime as the average transit time of matter through the reservoir, and its calculation need not be arbitrary." It is pointed out that there are a number of other characteristic timescales that can be used for describing the turnover of reservoirs, e.g., adjustment time, mean transit time, and expected lifetime (e.g., O'Neill et al. 1994). The point we make is that these timescales, though theoretically satisfying, all require a knowledge of impulse response behavior for $t \rightarrow \infty$. This can be worked around by a proper choice of an asymptote, and by using a "sufficiently long time series", but there is not a particularly good reason to trust very long simulations as much as short ones. Physical and numerical uncertainty must begin to dominate at some point. Nevertheless, this technical issue is also irrelevant to our point about the biosphere. Further, it was admittedly not the long time scale $\mathrm{CO}_{2}$ removal processes that were the focus of our discussion.

Third, they point out that "timescales describing the decay of excess $\mathrm{CO}_{2}$ mass in the atmosphere are not equivalent to those describing the decay of a single pulse in emissions." They go on to say that it therefore would probably not be reasonable to use $T_{1 / 2}$ in any kind of GWP calculation. We used the decay curve of the current load of excess $\mathrm{CO}_{2}$ as a metric with which to investigate certain dependencies related to model sensitivity and the representation of a direct- $\mathrm{CO}_{2}$ fertilization flux. In our conclusion, we mentioned that "one could envision" a modified GWP that was based on Half-Life values for the radiatively important trace gases. There is no reason why this kind of GWP could not be calculated, but it would, we agree, be based on a different set of concerns (namely, the short timescales). 
The fourth and final point is that "nonlinearities in the biospheric uptake may make the lifetime of $\mathrm{CO}_{2}$ even more uncertain than generally assumed, thus complicating the calculation of GWPs." This statement is supported by our work. Specifically because of these nonlinearities we avoid unrealistic pulse-based calculations. Further, understanding the nonlinear behavior of the terrestrial biosphere's role in the carbon cycle is an important factor in understanding future climate changes related to human activity. For example, if there are terrestrial sink mechanisms operating whose strength has been increasing (either due directly to increases in $\left[\mathrm{CO}_{2}\right]$ or other factors), and if those mechanisms begin saturate, then atmospheric $\mathrm{CO}_{2}$ growth rates could potentially increase anomalously. Indeed, with respect to nitrogen and $\mathrm{CO}_{2}$ fertilization, it has been suggested that saturation of the suspected terrestrial mechanisms could be close to this point at present (see Section 2.2). Explication of these terrestrial processes is important for making predictions. In Section 2.5 we will take a closer look at the implications of this possible phenomenon.

We assert that there is a difference in perspective, rather than opinion, with respect to the issues raised by Gaffin et al. (1995). In the political discussion surrounding the calculation of GWPs, it is commonly thought that low values for lifetimes of excess $\left[\mathrm{CO}_{2}\right]$ mean that carbon emissions are "not important", and conversely, high values mean that there is a longer commitment to an enhanced greenhouse effect, and thus they are "important". This concern has been cast more formally by the statement that measures such as $T_{1 / 2}$ only capture the short term dynamics of an intrinsically long timescale phenomenon (Caldeira and Kasting 1993). Our discussion (Moore and Braswell 1994) was somewhat orthogonal to the issue of the absolute magnitude of the indicator. We sought to make two points: (1) understanding the present and future behavior of the terrestrial biosphere is important for understanding the global carbon cycle and climate; and (2) the calculation of the lifetime of excess atmospheric $\mathrm{CO}_{2}$ (e.g., 
Lashof and Ahuja 1990, Shine et al. 1990) is somewhat arbitrary, subject to numerical difficulties associated with the multiple time scales of carbon cycle reservoirs.

The 1994 IPCC assessment (Albritton et al. 1995) contained some significant changes in the presentation of GWP indices from the earlier assessment (Shine et al. 1990). Carbon dioxide remained the reference gas in the calculation (Equation 2.5), but its lifetime was based on an integration of a balanced carbon cycle model instead of a three-parameter fit to an unbalanced ocean-atmosphere model. By balanced, we mean that the model reproduced the historic atmospheric $\mathrm{CO}_{2}$ trajectory, which is accomplished by including a mechanism for removing "missing" carbon. For example, the E-12B model (Section 2.3) is balanced with the inclusion of a fertilization flux parameterization. The inclusion of a terrestrial component. and the direct integration avoids the problems discussed by Moore and Braswell (1994). As in the previous assessment the GWPs depend upon the user-defined "integration time horizon". The changes also included improved estimates of the lifetimes of other trace gases. Many of the GWPs were higher, primarily because of the change in representation of the $\mathrm{CO}_{2}$ impulse response function. For example, the GWPs for nitrous oxide at 20. 100, and 500 years changed from 270,290 , and 190 , respectively. to 290,320 , and 180 , respectively.

\subsection{Stabilization Scenarios and Model Sensitivity}

In the first IPCC assessment, a number of scenario-based calculations were made using an ocean-atmosphere carbon cycle model. under the common assumption (at that time) of a net neutral biosphere in the extra-tropics. These scenarios reflected a commonly asked question: given estimates of future levels of industrial activity as predicted by econometric models, what will be the likely increase in the atmospheric concentration of $\mathrm{CO}_{2}$ and other trace gases? And, given that trajectory, what will be the associated change in radiative forcing of climate? Not 
surprisingly, the "business-as-usual" scenario (relatively modest or nonexistent controls on emissions, and heavy deforestation in the tropics) yielded a significant global warming of about $4^{\circ} \mathrm{C}$. The most "optimistic" scenario (shifting to renewable and nuclear energy, with tougher controls on emissions and less deforestation) still resulted in increasing $\mathrm{CO}_{2}$ concentrations and a warming of about $2.5^{\circ} \mathrm{C}$. It was clearly demonstrated that stabilizing emissions is a very different issue than stabilizing concentrations in the atmosphere (the former is related to the derivative of the latter), and that in the absence of stable concentrations, the world is likely committed to unpredictable and uncontrollable changes in the climate system.

In the second IPCC assessment, the community reported on an effort that was designed to look at the question of stabilizing atmospheric concentrations of $\mathrm{CO}_{2}$ (Enting et al. 1994. Schimel et al. 1995). A number of different models were exercised that included oceanic and terrestrial processes, but in varying degrees, and with varying complexity. The following constraints were applied to all participating model simulations: (1) they must approximately reproduce the atmospheric record (a spline fit to ice core and direct measurements) of $\mathrm{CO}_{2}$ change since the late 18th Century: (2) they must follow a prescribed trajectory to stabilization of $\left[\mathrm{CO}_{2}\right]$ (Fig. 2.16); (3) additional terrestrial sink mechanisms must be linked to atmospheric $\mathrm{CO}_{2}$ (i.e., direct fertilization); and (4) net biotic emissions due to land-use change in the tropics are prescribed, and fall linearly to zero from 1990 to 2100 . The task was to calculate by inversion (or "deconvolution") the future fossil fuel $\mathrm{CO}_{2}$ flux that would be required to stabilize $\left[\mathrm{CO}_{2}\right]$ at $450 \mathrm{ppm}$, and at $650 \mathrm{ppm}$ (Fig. 2.16).

Figure 2.17 shows the fossil fuel emissions predicted by the Moore and Braswell (1994) model (E-12B) that were used in the intercomparison. The overall shape of the curve is very similar to that predicted by the other models (not shown). It is apparent that, according to the model, stabilization at either $450 \mathrm{ppm}$ or $650 \mathrm{ppm}$ would require a substantial decrease in 
fossil $\mathrm{CO}_{2}$ emissions. In fact. this trajectory lies below even the most "optimistic" scenario mentioned above. This fact is cause for concern because that scenario requires a substantial set of controls operating against free market pressures. as well as a large shift toward nuclear energy, which some would argue carries its own set of environmental problems.

In this deconvolution. we used the logistic representation of the direct $\mathrm{CO}_{2}$ fertilization effect (Fig. 2.17), and allowed it to operate for the entire time. Consequently, it can be seen that the allowed emissions eventually stabilize at 1980 and 1950 levels for S650 and S450. respectively, when the fertilization flux balances the fossil flux. It is widely believed that terrestrial mechanisms which give rise to enhanced production or storage of carbon will not persist indefinitely because the ecosystems will acclimate, or perhaps decline. This is a very uncertain area that is the subject of a great deal of research at present. To explore the consequences of this effect or effects, we ran another simulation in which the fertilization term was linearly decreased from its value in 1990 to zero in the final year. 2300 (Fig. 2.18). It can

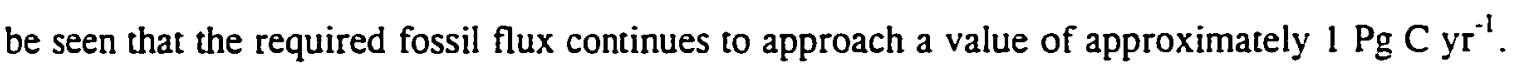
This was meant only as an analysis of the sensitivity, but the requirement of very low emissions is the trivial result of approaching a net neutral biosphere.

Figure 2.19 shows the implications of the previous two calculations for the pool of carbon in terrestrial vegetation. In both cases, the carbon lost through deforestation-related activities is recovered within $\sim 100$ years. In the case of continuing enhanced sequestration (fertilization flux of about $1.5 \mathrm{PgC} \mathrm{yr}^{-1}$ ), the size of the terrestrial vegetation stock increases by about $500 \mathrm{Pg} \mathrm{C}$ during the final two hundred years, an amount roughly equal to estimates of the growth of the biosphere (including soils) from the last glacial maximum $(10,000 \mathrm{BP})$ to the present (Bird et al. 1994). The continuing storage in living plant tissue to the extent shown is probably unrealistic. With a fertilization effect that is mitigated by (unspecified) negative 


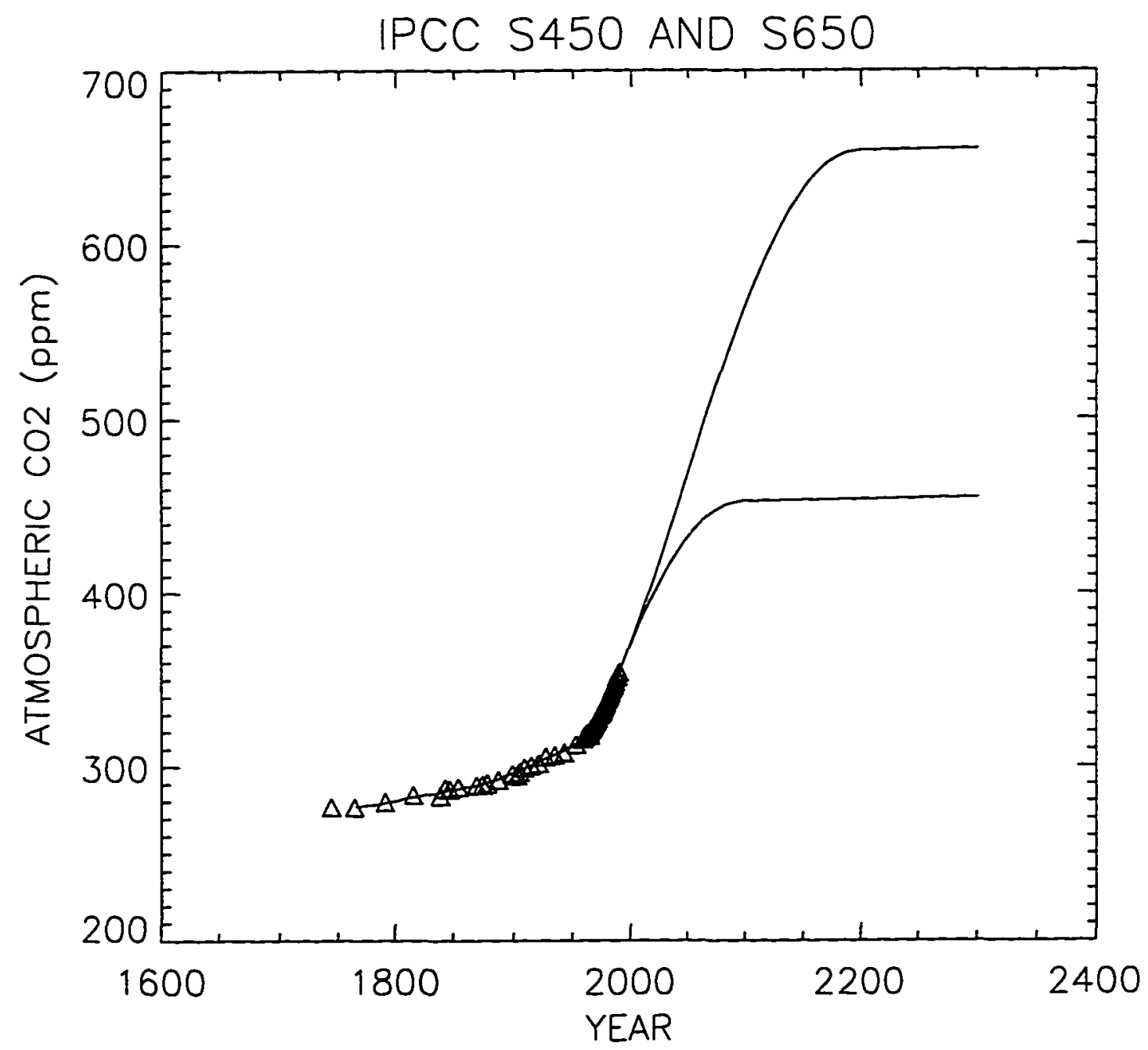

Figure 2.16 IPCC scenarios for prescribed stabilization of atmospheric $\mathrm{CO}_{2}$ concentrations at 450 and $650 \mathrm{ppm}$. Triangles represent the ice core and direct measurements. 


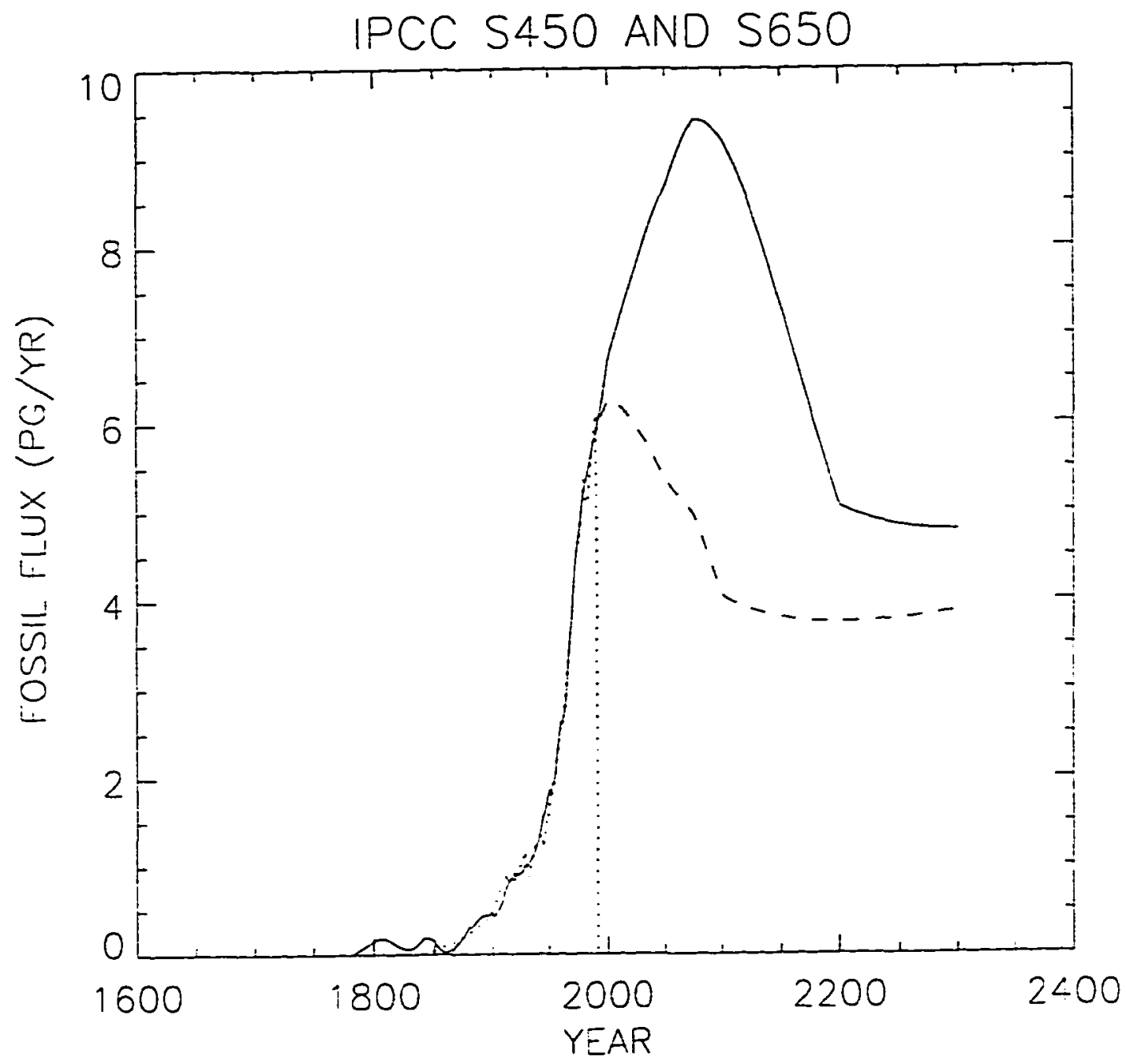

Figure 2.17 Deconvolved fossil fuel flux, using the Moore and Braswell (1994) model, required to achieve stabilization shown in Fig. 2.16 under the assumption that deforestation emissions decline linearly to zero from 1990 to 2300 (a requirement of the IPCC intercomparison), and that enhanced terrestrial sink mechanisms continue into the future. The enhanced sink stabilizes because of the asymptotic dependence of the fertilization flux. 


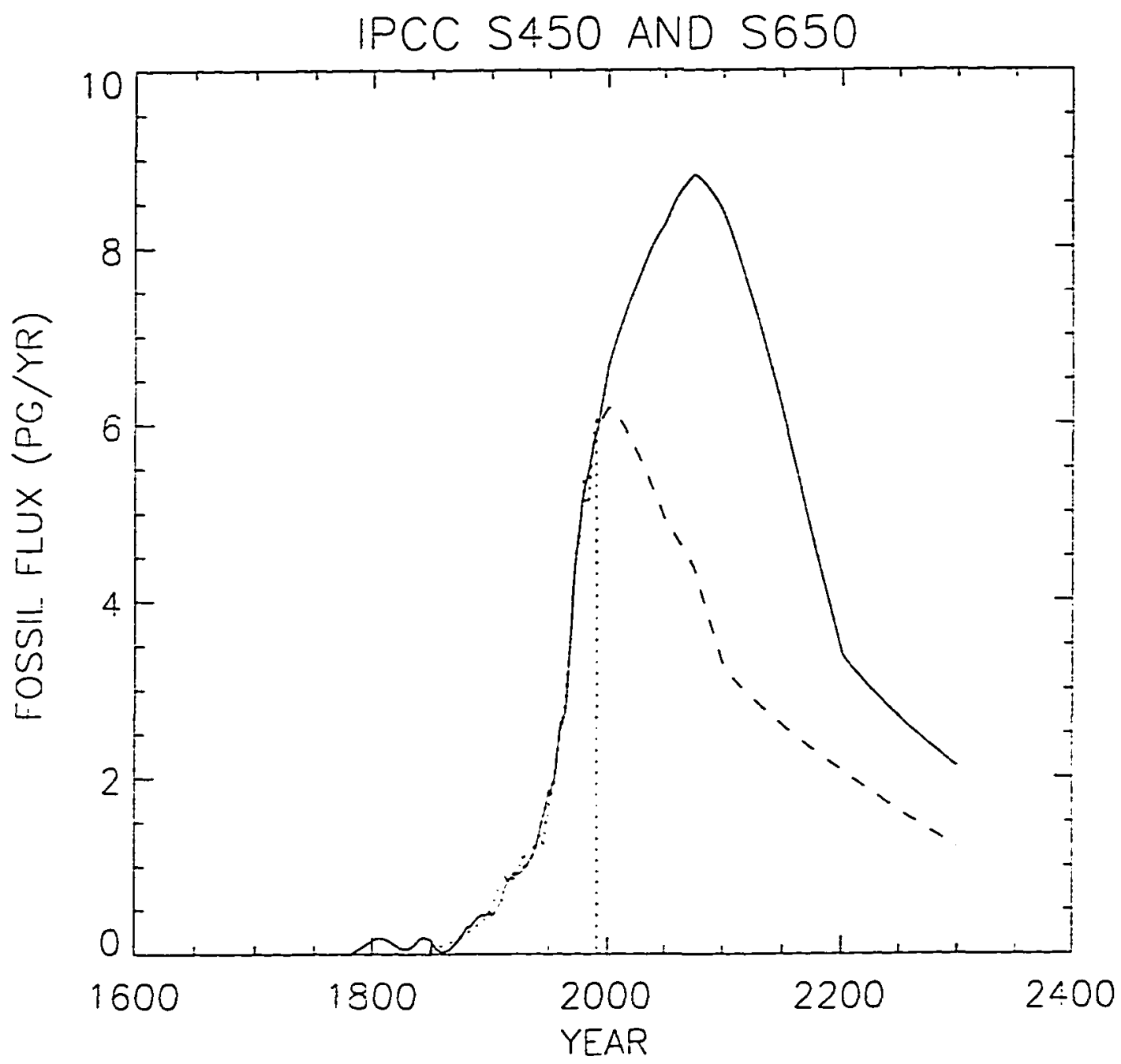

Figure 2.18 The same as Fig. 2.17, except that the fertilization flux is reduced to zero linearly from 1990 to 2300 . This tests the sensitivity to the notion that negative feedbacks are mitigating the fertilization of the biosphere. 
feedbacks, a more modest regrowth is realized (about $100 \mathrm{Pg} \mathrm{C}$ ). This result, combined with ecological evidence that the feedbacks exist (see Section 2.2) suggests that the lower fossil flux projections (Fig. 2.18) may be a more realistic (but more difficult) path to stabilization of atmospheric $\left[\mathrm{CO}_{2}\right]$.

The importance of assumptions about the terrestrial biosphere can be expressed in another way. We applied the deconvolved fossil flux in the case of continuing fertilization (Fig. 2.17 ) to a forward simulation ( $\mathrm{S} 650$ ) of the $\mathrm{E}-12 \mathrm{~B}$ model. Without changing any model parameters the forward simulation would simply reproduce the $\mathrm{S} 650$ atmospheric $\mathrm{CO}_{2}$ concentrations (Fig. 2.20, dashed line). Eliminating the fertilization flux, however, results in concentrations at the end of the simulation that are about $100 \mathrm{ppm}$ greater and that drift slowly upward (Fig. 2.20, solid line).

Finally, it has been pointed out that since the capacity for the oceans to remove excess $\mathrm{CO}_{2}$ is reduced at higher $\left[\mathrm{CO}_{2}\right]$, characteristic time scales (Section 2.4) should be calculated using the decay of a pulse of $\mathrm{CO}_{2}$ added to a scenario-based simulation such as $\mathrm{S} 650$ (Caldeira 1993). Figure 2.21a shows the resulting trajectory for 500 years following a $10 \mathrm{Pg} \mathrm{C}$ instantaneous addition of $\mathrm{CO}_{2}$ to the atmosphere at $t=1990$. The lower panel (Fig. 2.21b) is a closer view of the first 100 years. As discussed earlier (Section 2.2), multiple time scales for the removal of $\mathrm{CO}_{2}$ are apparent, resulting from the turnover of the surface oceans, terrestrial vegetation, and soils. In contrast to the previous sensitivity exercises (Figs. 2.17-2.20), this particular diagnostic is invariant with respect to an active biosphere because in our model, the "donor-control" response of terrestrial productivity is relatively insensitive to small (e.g., 10 $\mathrm{Pg} \mathrm{C}$ ) changes in $\left[\mathrm{CO}_{2}\right]$ at current levels and higher because of the asymptotic nature of the assumed sink processes (Fig. 2.11). This difference highlights the potential for confusion in projecting the future of the carbon cycle, and suggests that care must be taken in constructing 


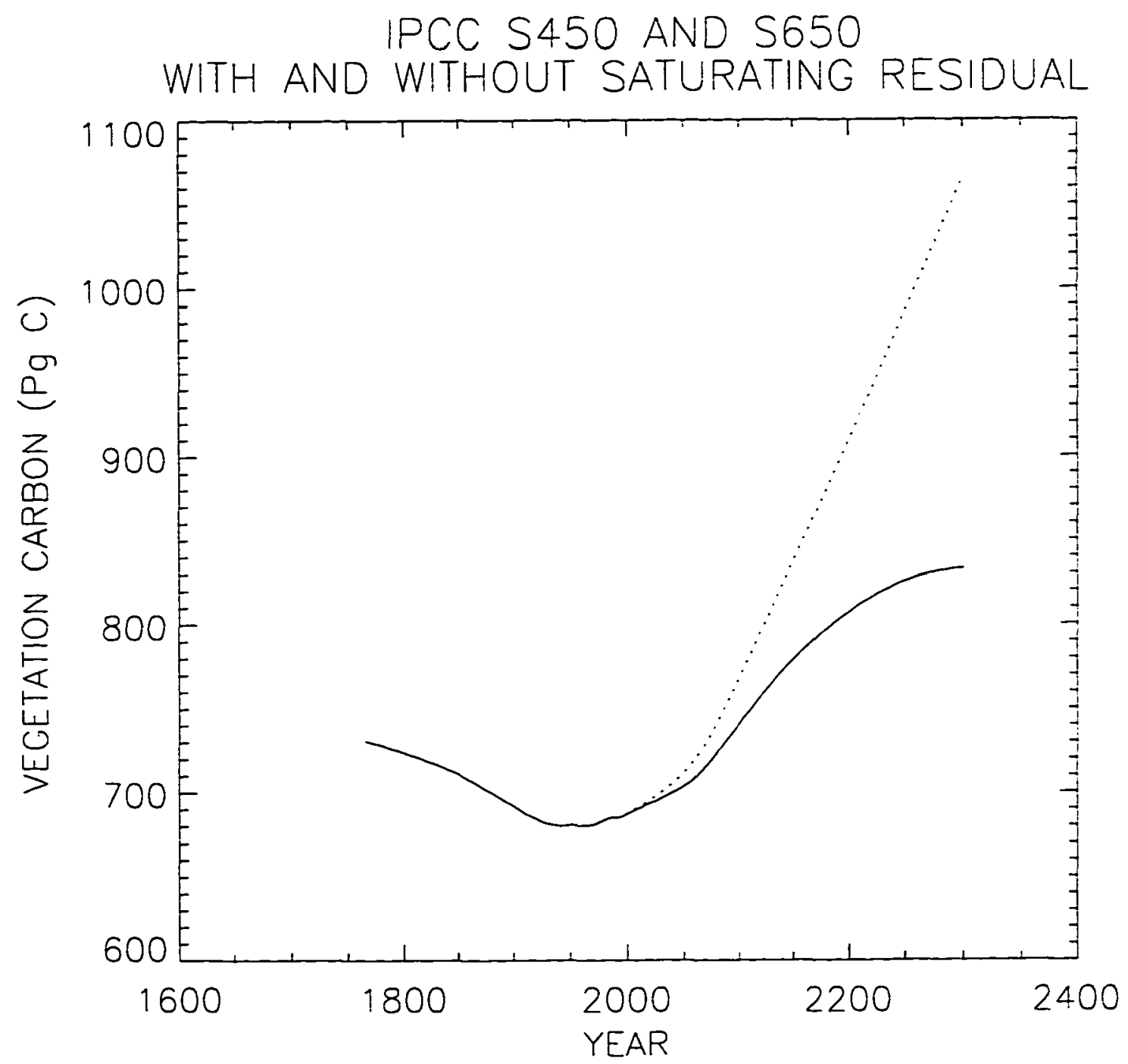

Figure 2.19 The pool of carbon in living vegetation for the simulations shown in Figs. 2.172.18 . 


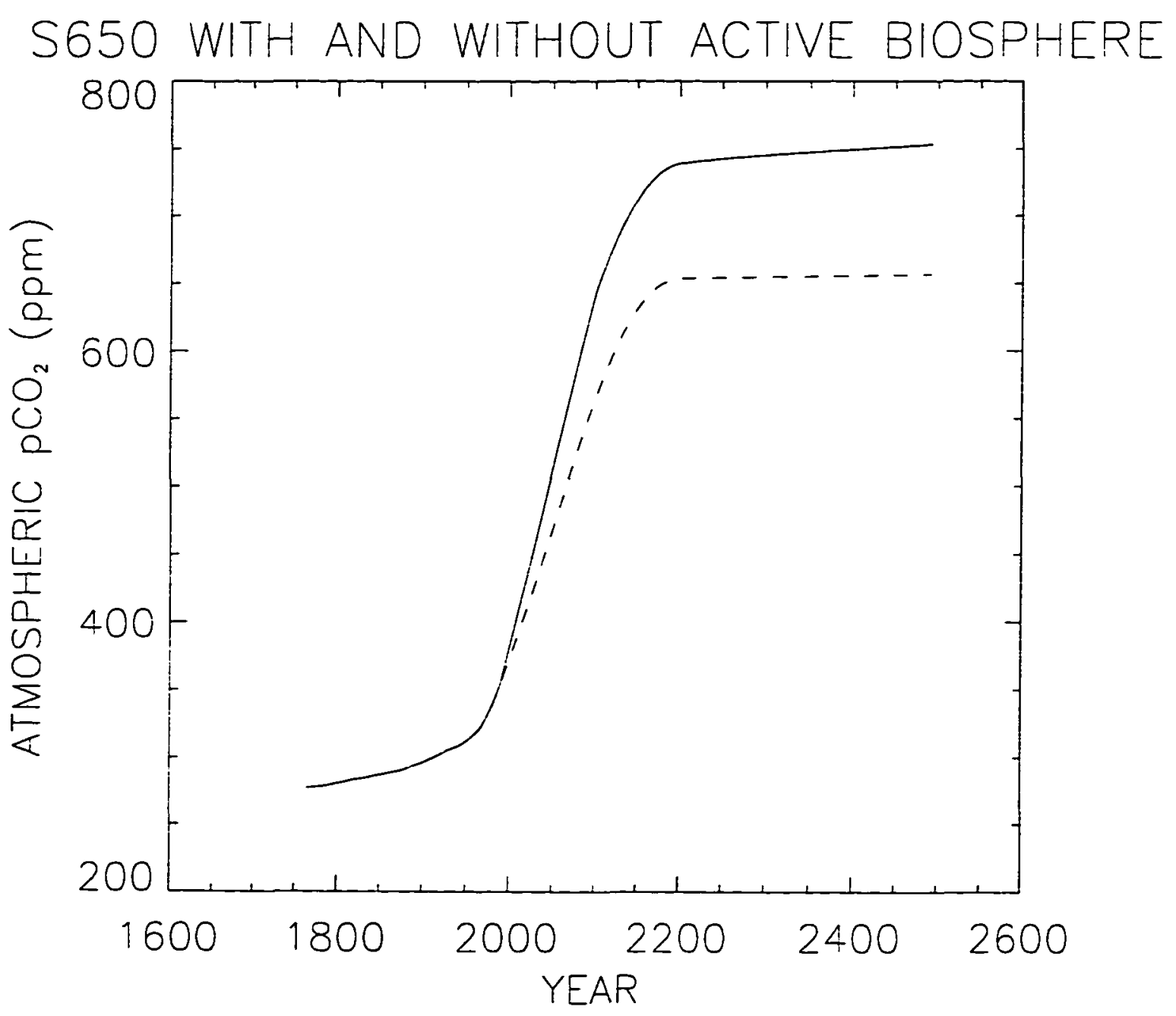

Figure 2.20 The effect of the inclusion of $\mathrm{CO}_{2}$ fertilization in modeling the stabilization. The lower line is the same as Fig. 2.16. The upper line shows the results of a forward integration of the model using as input the deconvolved flux shown in Fig. 2.17, except with fertilization deactivated. 

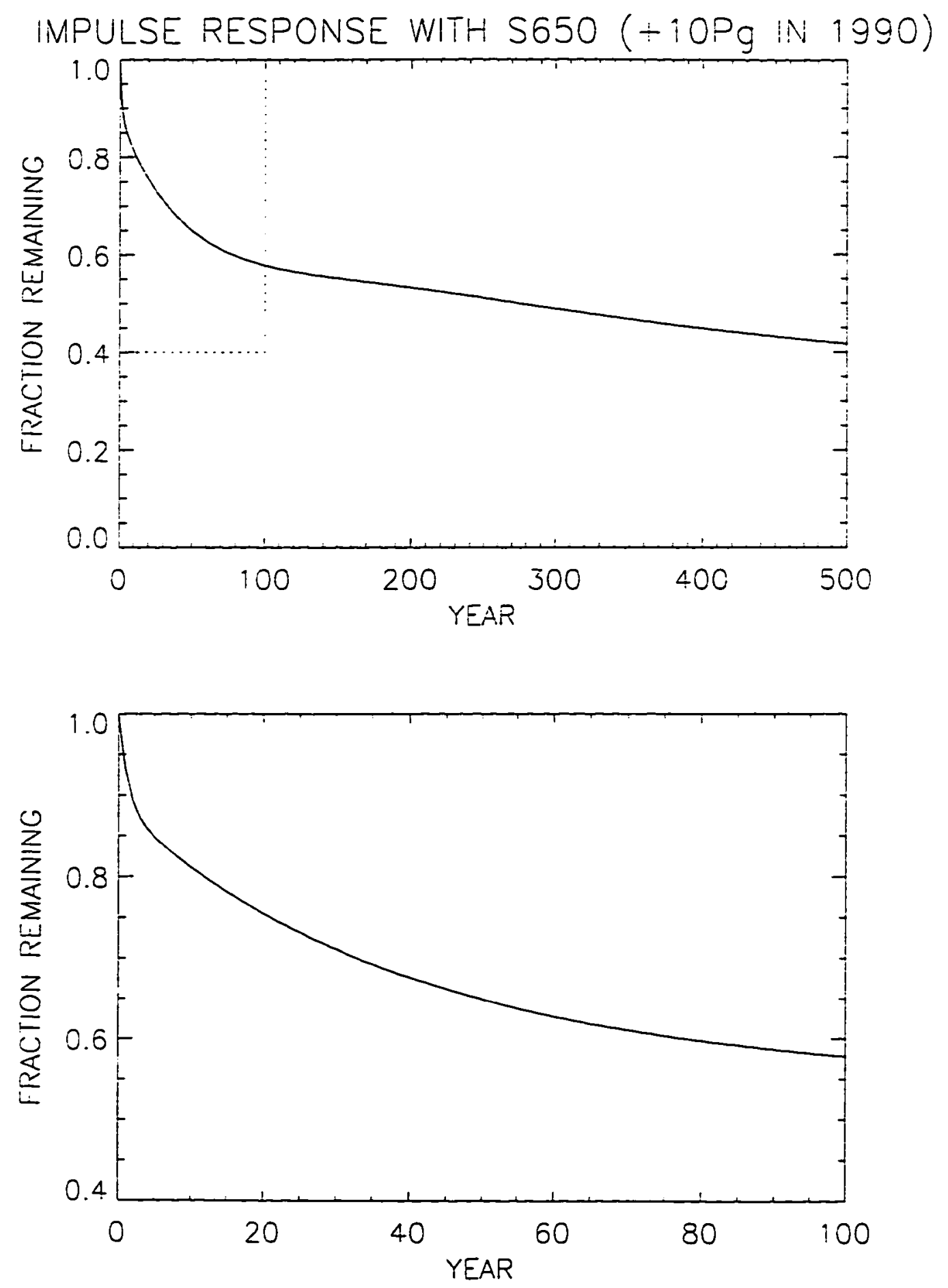

Figure 2.21 The decay of a $10 \mathrm{Pg} \mathrm{C}$ pulse of $\mathrm{CO}_{2}$ added to the model atmosphere in the year 1990 for an $\mathrm{S} 650$ stabilization simulation. The curve is obtained by subtracting the perturbed model output from the $\mathrm{S} 650$ concentrations (Fig. 2.16). 
and assessing indicators of the lifetime of excess atmospheric $\mathrm{CO}_{2}$.

\subsection{Summary}

The global carbon cycle is an interactive component of the Earth's climate system, and anthropogenic alteration of the patterns of global $\mathrm{CO}_{2}$ fluxes (and other trace gases) will likely result, and perhaps has already resulted, in systematic changes in air temperature, precipitation, and sea level. The magnitude, timing, and distribution of these changes is highly uncertain. The state and behavior of terrestrial ecosystems are, in turn, a complex and important part of the global carbon cycle. Indeed, a great deal of the uncertainty in future concentrations of atmospheric $\mathrm{CO}_{2}$ rests on uncertainty of the aggregate behavior of vegetation-soil-atmosphere systems worldwide. Thus, one of the great challenges to understanding the carbon cycle is integrating mechanistic knowledge of physical, chemical, and ecological processes at the local scale with global atmospheric measurements.

Numerical modeling is a tool that is at the forefront of carbon cycle research. Highly aggregated models of the carbon cycle (e.g., Bolin et al. 1983, Emanuel et al. 1984, Moore and Braswell 1994) are useful because they incorporate simple rules for the transformation of inorganic and organic carbon throughout the Earth system, allowing integration over long time scales. The observed distribution of tracers (principally ${ }^{14} \mathrm{C}$ ) forms the basis for the calibration of turnover rates of various ocean and soils compartments, while vegetation turnover is linked to plant lifespan. Analyses using these dynamic models provide, at least, a consistency check on our understanding of the terms in the carbon budget and, at most, a means of generating realistic predictions and policy-relevant inquiries that are driven by models of sociological and economic activity. 
Atmospheric measurements have also played a central role in the understanding of the carbon cycle, beginning with the observation that $\left[\mathrm{CO}_{2}\right]$ was in fact increasing in the atmosphere (Keeling et al. 1976). Direct $\left[\mathrm{CO}_{2}\right]$ assays and the well known patterns of fossil fuel emissions have been the most powerful constraints on the construction of global carbon budgets. Other measurements of atmospheric composition, including both carbon isotopes and oxygen, have added further detail to carbon cycle calculations. Both carbon-13 and oxygen (associated with $\mathrm{CO}_{2}$ and organic matter) have different exchange ratios (relative to ${ }^{12} \mathrm{C}$ ) for the oceans and terrestrial biosphere. These "signatures" allow for estimates of partitioning of net carbon flux between these two reservoirs. In addition, attempts to understand interannual variability in atmospheric $\mathrm{CO}_{2}$ levels (which can be large, on the order of $1-2 \mathrm{Pg} \mathrm{C}$ ) can lead to a better understanding of the carbon cycle at the mechanistic level. This understanding translates into a more clear picture of the anthropogenically altered carbon budget.

The major terms (fluxes) in the global carbon budget are qualitatively well-known (Table 2.1): the net atmospheric increase, fossil fuel emissions. net ocean atmosphere exchange, and net terrestrial-atmosphere exchange. Of these, the terrestrial term is most uncertain, and consists of a number of potentially interacting processes, including carbon emissions from land-use change (primarily in the tropics), carbon sequestration due to regrowth of abandoned agricultural land (in the tropics, but also likely in the northern temperate latitudes), enhanced carbon assimilation and storage by vegetation and soils "fertilized" by industrial sources of nutrients and/or increased $\left[\mathrm{CO}_{2}\right]$, and perhaps, altered patterns of terrestrial carbon storage arising from changes in climate. The high degree of spatial heterogeneity and temporal variability of the terrestrial terms arise from both natural and human factors. Improved knowledge of these mechanisms has resulted in a more correct, but more uncertain picture of the balance of global $\mathrm{CO}_{2}$ fluxes (Table 2.1). 
We have demonstrated in this chapter that terrestrial processes, both natural and anthropogenic, greatly influence the behavior of $\mathrm{CO}_{2}$ in the atmosphere, and thus the commitment to enhanced greenhouse warming. The complexity of the terrestrial biosphere as a whole (in terms of carbon) arises from spatial heterogeneity in the distribution of plants, soils, and climate, dynamics at the local scale that are highly interactive with other biogeochemical cycles, and the possibility of multiple direct and indirect effects of human intervention. In later chapters we will present analyses incorporating models and data that highlight possible directions for improvement of estimates of the spatial-temporal patterns of terrestrial net ecosystem production. These areas include a focus on interaction with atmospheric chemistry (e.g., ecosystem effects of pollutant deposition; Chapter 3). internal ecosystem regulation (Chapter 4), and integration with satellite remote sensing measurements (Chapter 4 and Chapter 5). The motivation for these studies is derived from questions raised about the interactive role of the global carbon cycle in the global climate system. 


\section{CHAPTER 3}

\section{ATMOSPHERIC NITROGEN DEPOSITION AND THE GLOBAL CARBON CYCLE}

\subsection{Introduction}

The global cycles of carbon and nitrogen are coupled by the biosphere. This connection is one of the most important of all global biogeochemical phenomena, linking atmospheric chemistry with terrestrial and aquatic processes (Schlesinger 1991. Prinn 1994. Asner et al. In press). On both land and ocean, assimilation of carbon by photosynthesis is associated with uptake of mineral nitrogen, and decomposition of dead organic matter results in the release of nitrogen to the soil environment. Conversely, inputs (e.g., fixation and deposition) and losses (e.g., leaching and denitrification) of nitrogen to/from ecosystems impact net production and carbon storage because of the widespread limitation of ecosystems by the availability of nitrogen (Vitousek and Howarth 1991). The exogenous fluxes of $\mathbf{N}$ (e.g., atmospheric deposition) are typically small compared to the amount of $\mathrm{N}$ that is internally recycled and they can be highly variable in space and time, but they play a significant role in determining site nutrient status over annual to millennial time scales. This is because most terrestrial nitrogen is tied up in organic matter rather than in mineral form available to plants (Sprent 1987).

Human activities associated with industrialization and expansion of urban/agricultural centers are resulting in an increased input of chemically reactive nitrogen species into the atmosphere. These compounds represent a perturbation to the natural nitrogen cycle and originate primarily as: (1) nitrogen oxides from fossil fuel combustion, biomass burning, and disturbed soils; and (2) volatilization of ammonia from livestock wastes and fertilizer $\mathrm{N}$ (fixed 
from $\mathrm{N}_{2}$ using the Haber-Bosch process). Recent estimates indicate that anthropogenic fixation of nitrogen now exceeds natural fixation (Galloway et al. 1995). These short-lived "excess" nitrogen compounds participate in complex atmospheric chemistry and transport, and are deposited downwind from their source in particulate or ionic forms that are readily available for plant and microbial uptake.

The disruption of the pre-industrial $\mathrm{N}$ cycle is analogous to the anthropogenic alteration of the carbon cycle. For example, the atmospheric concentration of nitrous oxide $\left(\mathrm{N}_{2} \mathrm{O}\right)$, a long-lived radiatively active trace gas that has significant biogenic sources, though it has been rising steadily over the last $\sim 200$ years, remained approximately constant for at least 2000 years prior to the onset of increases (Khalil and Rasmussen 1988, Watson et al. 1990). Thus, we use the same assumption for $\mathrm{N}$ as in Chapter 2 (for carbon) that the pre-industrial cycling of nitrogen between the major reservoirs was in approximate balance. This assumption is the basis for using a perturbation model to study the fate of excess fixed carbon resulting from $\mathrm{N}$ deposition.

In this chapter we explore one aspect of the coupling of the carbon and nitrogen cycles and their perturbation by human activities: fertilization of the terrestrial biosphere by anthropogenic nitrogen deposition. In Section 3.2 (based on Townsend et al. 1996) we estimate the extent to which deposition of nitrogen on terrestrial ecosystems (primarily Northern Hemisphere forests) has resulted in a significant sink for atmospheric carbon dioxide in recent decades (see also Chapter 2). This estimate is derived using a simple, dynamic, and geographically referenced model of terrestrial $\mathrm{C}$ and $\mathrm{N}$ cycling. We include a sensitivity test of the estimate to assumptions about $\mathrm{C}: \mathrm{N}$ ratios and the allocation of excess carbon to tissues of various lifetimes. In Section 3.3 (based on Holland et al. In press), we look at the sensitivity of our model estimate to the spatial patterns of deposition and to assumptions related to the portion 
of biotic emissions that result from anthropogenically-driven changes. Further, because chronic nitrogen deposition is associated with other factors (e.g., high ozone levels and acidification) which lead to removal of $\mathrm{N}$-limitation, or possibly ecosystem decline, we explore model sensitivity to the "N-saturation" effect using a simple parametric framework (Section 3.4).

\subsection{Spatial And Temporal Patterns in Terrestrial Carbon Storage due to Deposition of Fossil Fuel Nitrogen ${ }^{\dagger}$}

\section{$\underline{3.2 .1 \text { Introduction }}$}

Recent model calculations which balance $\mathrm{CO}_{2}$ accumulation in the atmosphere against fossil fuel release, the effects of changing land-use, and oceanic uptake, calculate a substantial residual term, sometimes referred to as a "missing sink" (Tans et al. 1990, Siegenthaler and Sarmiento 1993; see also Chapter 2). Carbon uptake by the terrestrial biosphere is likely a major component of the calculated residual flux. Possible mechanisms behind this increased uptake include: (1) elevated $\mathrm{CO}_{2}$ stimulating photosynthesis (e.g., Bazzaz 1990), (2) climate variability (e.g., Dai and Fung 1993), and (3) the issue addressed here: increased carbon fixation resulting from widespread deposition of nitrogen (e.g., Peterson and Melillo 1985).

Industrial and agricultural activity in recent decades has caused a considerable increase in emissions of reactive $\mathrm{N}$ to the atmosphere (Galloway et al. 1995). The primary sources of this excess $\mathrm{N}$ are the combustion of fossil fuels, the manufacture and use of fertilizers, livestock, and burning of biomass as a result of changing land use practices. The primary forms are either nitrogen oxides $\left(\mathrm{NO}_{\mathrm{x}}=\mathrm{NO}+\mathrm{NO}_{2}\right)$ or ammonia $\left(\mathrm{NH}_{3}\right)$. Both forms are reactive in the lower atmosphere (Logan et al. 1981), and all $\mathrm{NO}_{\mathrm{x}}$ and $\mathrm{NH}_{3}$ emitted will be deposited back onto land or sea, with the ratio of deposited to emitted $\mathbf{N}$ decreasing rapidly with distance from

\footnotetext{
${ }^{\dagger}$ Adapted from the article by A.R. Townsend, B.H. Braswell, E.A. Holland, and J.E. Penner, Ecological Applications 6:806-814, 1996. Copyright by the Ecological Society of America.
} 
the source of emissions (Penner et al. 1991). Both families of $\mathrm{N}$ species are highly reactive in the troposphere. Thus, emitted $\mathrm{NO}_{\mathrm{x}}$ can be deposited in a variety of forms, collectively termed $\mathrm{NO}_{y}\left(\mathrm{NO}_{\mathrm{x}}+\mathrm{HNO}_{2}+\mathrm{HNO}_{3}+\mathrm{HO}_{2} \mathrm{NO}_{2}+\mathrm{NO}_{3}+2 \mathrm{~N}_{2} \mathrm{O}_{5}+\right.$ peroxyacetyl nitrate $\{$ PAN $\}+$ particulate nitrates). Similarly, total ammonium deposition is as $\mathrm{NH}_{\mathbf{x}}\left(\mathrm{NH}_{4}{ }^{+}+\mathrm{NH}_{3}+\right.$ $\mathrm{NH}_{4} \mathrm{SO}_{4}$ ).

Several authors have pointed out that enhanced $\mathbf{N}$ deposition could stimulate a significant increase in terrestrial carbon uptake (Peterson and Melillo 1985, Schindler and Bayley 1993). The basis of this hypothesis is twofold: terrestrial productivity is typically $\mathrm{N}$ limited (Vitousek and Howarth 1991), and carbon to nitrogen ratios in terrestrial vegetation are high, so that large amounts of $\mathrm{C}$ may be fixed for every unit of $\mathrm{N}$. There is considerable uncertainty, however, with respect to both the magnitude and persistence of any $\mathrm{N}$-derived carbon sink. Peterson and Melillo (1985) estimated a total terrestrial sink of only $0.1 \mathrm{Pg} \mathrm{C} \mathrm{yr}^{-1}$, while Schindler and Bayley (1993) suggested that annual storage may be in excess of $1 \mathrm{Pg} \mathrm{C}$. Excess carbon uptake due to $\mathrm{N}$ deposition in any given year will be the net flux, equal to the net primary production stimulated by the current year's deposition minus the respiration from excess carbon fixed in that and previous years. In other words, to estimate the current year's carbon uptake, one must know the amount of respiration from carbon pools formed due to $\mathrm{N}$ inputs in the past.

It is also important to consider strong spatial gradients in the factors that influence terrestrial carbon uptake. $\mathrm{N}$ deposition to terrestrial systems can vary from less than 1 to greater than $80 \mathrm{~kg} \mathrm{~N}^{-1} \mathrm{yr}^{-1}$ (Berendse et al. 1993, Galloway et al. 1995). Furthermore, different ecosystem types vary greatly in their potential for carbon sequestration. Woody tissue can have C:N ratios in excess of 300 and lifetimes greater than 100 years (Vitousek et al. 1988, Schimel et al. 1994), therefore any excess $\mathrm{N}$ which stimulates wood production will cause a 
relatively large and long-term removal of $C$ from the atmosphere. In contrast, foliar biomass in forests and grasslands in general have much narrower $\mathrm{C}: \mathrm{N}$ ratios $(30-80)$ and shorter tissue lifetimes, and therefore have a more limited capacity for carbon storage. Soil organic matter (SOM) also contains fractions that turn over slowly (e.g., Schimel et al. 1994), but with C:N ratios that are somewhat narrower than foliage. Thus, the extent to which soils can represent a $\mathrm{C}$ sink due to $\mathrm{N}$-fertilization will depend in part on how much excess $\mathrm{N}$ is deposited on ecosystems that form SOM rapidly (e.g., temperate grasslands), but also on the fraction of additional $C$ that ends up in the "slow" SOM pools.

Deposition onto cultivated areas is not likely to contribute to a terrestrial sink, for a number of reasons. First, most cultivation practices cause soil carbon stocks to decline with time (Baur and Black 1983, Burke et al. 1989, Davidson and Ackerman 1993). Second, crops tend to have relatively narrow $\mathrm{C}: \mathrm{N}$ ratios, and will also be harvested and consumed, therefore they cannot be a long-term reservoir. Finally, most agricultural lands already receive high inputs of $\mathrm{N}$ from fertilizer and cultivated legumes, thus additional inputs from deposition are not likely to stimulate further $C$ uptake.

The objective of this study was to estimate a likely range for terrestrial carbon storage resulting from deposition of fossil fuel $\mathrm{NO}_{\mathrm{x}}$ emissions, including its spatial and temporal distribution. Our estimate is based on a simple perturbation model of terrestrial biogeochemistry (NDEP: Nitrogen Deposition / Ecosystem Production), and has three important features which distinguish it from other calculations of this type. First, we accounted for spatial variability in potential carbon storage by using simulated $\mathrm{N}$ deposition rates from an atmospheric transport model together with a map of vegetation types and land-use distributions. Second, the model allocates carbon and nitrogen to vegetation and soil pools of different 
turnover times and $\mathrm{C}: \mathrm{N}$ ratios. Finally, NDEP estimates the effects of prior $\mathrm{N}$ deposition on the current year's carbon exchange.

We initially restricted our analysis to fossil fuel $\mathrm{N}$ for several reasons and, as such, this analysis provides possibly a conservative estimate. Fossil $\mathrm{NO}_{\mathrm{x}}$ is the only source of deposition for which we can obtain a reasonable estimate of temporal trends. Also, fossil fuels are the largest source of oxidized $\mathrm{N}$ to the atmosphere; our estimate for fossil fuel deposition to land is $17 \mathrm{Tg} \mathrm{N} \mathrm{yr}^{-1}$ (1990 value); Levy et al.'s (In press b) estimate for $\mathrm{NO}_{\mathrm{y}}$ deposition from all sources is $28 \mathrm{Tg} \mathrm{N} \mathrm{yr}^{-1}$. The difference between these two numbers is predominantly due to biomass burning $\left(-8 \mathrm{Tg} \mathrm{N} \mathrm{yr}^{-1}\right)$, which is concentrated in tropical areas where $\mathrm{N}$ limitation of plant growth is not as widespread (Vitousek and Sanford 1986). Thus, deposition of this additional oxidized $\mathrm{N}$ is less likely to stimulate carbon uptake and storage (but see Section 3.3).

The one potentially significant source of excess $\mathrm{N}$ not included in our analysis is ammonium $\left(\mathrm{NH}_{\mathrm{x}}\right)$ deposition. Galloway et al. (1995) estimate anthropogenic sources of $\mathrm{NH}_{\mathrm{x}}$ deposition on land to be $38 \mathrm{Tg} \mathrm{N} \mathrm{yr}{ }^{-1}$, therefore it appears to be the largest source of excess $\mathrm{N}$ deposition. and may stimulate significant carbon uptake. We deal with issues related to $\mathrm{NH}_{\mathbf{x}}$ deposition as well as $\mathrm{NO}_{\mathrm{y}}$ from other sources in Section 3.3.

\subsubsection{Methods and model description}

NDEP is a perturbation model, is based on the Century soil organic matter model (Parton et al. 1987. Schimel et al. 1994), and is designed to run at an annual time step and $1^{\circ} \times 1^{\circ}$ spatial resolution. Inputs for each cell are nitrogen deposition, the fraction of available $\mathrm{N}$ lost from the ecosystem, mean annual temperature $\left({ }^{\circ} \mathrm{C}\right)$, soil texture (\% clay), allocation of carbon fixed between woody and non-woody vegetation, and $\mathrm{C}$ to $\mathrm{N}$ ratios for vegetation, detritus, microbial biomass, and soil organic matter. Allocation and $\mathrm{C}: \mathrm{N}$ ratios were specific 
for each vegetation type (Table 3.1), and vegetation distributions were based on the map derived by Matthews (1983). Cultivation in the Matthews (1983) database ranges from 0-100\% intensity in $25 \%$ increments; for example, a grid cell with $50 \%$ intensity is represented as half cultivated land and half natural vegetation. The fraction of area under cultivation in any cell was excluded from our calculations. The model does not account for changes in vegetation distribution, allocation. or $\mathrm{C}: \mathrm{N}$ ratios with time.

Spatial distributions in $\mathrm{NO}_{\mathrm{y}}$ deposition were estimated using values from an annual run of the GRANTOUR atmospheric transport model (Penner et al. 1991; see also Section 3.3) interpolated from $4.4^{\circ} \times 7.5^{\circ}$ resolution to $1^{\circ} \times 1^{\circ}$. Temporal changes in $\mathrm{NO}_{\mathrm{y}}$ deposition were based on a simulation of fossil fuel $\mathrm{NO}_{\mathrm{y}}$ deposition for 1980 (Nevison 1994). For each grid cell, deposition was calculated over the period from $1845-1990$ by scaling the mapped value of $\mathrm{N}$ deposition to the global fossil fuel emissions time series.

A summary of the pools and fluxes in the NDEP model is shown in Fig. 3.1. Carbon and nitrogen pools in this perturbation model are those due to nitrogen deposition only. therefore they were set initially to zero. Changes in woody and non-woody carbon pools $\left(C_{\mathrm{w}}\right.$. $\left.C_{n w}\right)$ depend on the flux of available nitrogen $\left(N_{a v}\right)$, an allocation term $\left(f_{w}\right)$ which specifies the fraction of carbon going to woody biomass, the $\mathrm{C}: \mathrm{N}$ ratio of each pool, and the residence time of carbon in the pools:

$$
\begin{aligned}
& \frac{d C_{w}}{d t}=f_{w^{\prime}} \cdot C N_{w} \cdot N_{a v}-\alpha_{w} \cdot C_{w \cdot}, \\
& \frac{d C_{n w}}{d t}=\left(1-f_{w}\right) \cdot C N_{n w} \cdot N_{a v}-\alpha_{n w} \cdot C_{n w \cdot}
\end{aligned}
$$


Table 3.1 Biome-specific parameters used in the baseline estimate of $\mathrm{N}$ deposition effects on carbon storage. Parameters are defined in the text and shown in Fig. 3.1. Parameter values are estimates taken from the literature: $f_{w}$ (Nadelhoffer et al. 1985, Aber and Melillo 1991); $C N_{n w}, C N_{w}$ (Cole and Rapp 1981, Vitousek et al. 1988); $C N_{m i c}, C N_{\text {tret }} C N_{\text {stow }}$ (Schimel et al. 1994); $\alpha_{w,} \alpha_{n w}$ (Esser et al. 1993). The area for each biome is in millions of $\mathrm{km}^{2}$ (Matthews 1983).

\begin{tabular}{lccccccccc}
\hline Vegetation class & Area & $f_{w}$ & $C N_{m w}$ & $C N_{w}$ & $C N_{\text {mic }}$ & $C N_{\text {dlet }}$ & $C N_{\text {slow }}$ & $\alpha_{w}$ & $\alpha_{m w}$ \\
\hline Trop. ev. forest & 12.29 & 0.5 & 50 & 150 & 14 & 120 & 25 & 0.005 & 0.83 \\
Trop./sub. ev. seas. brl. for. & 3.29 & 0.5 & 50 & 150 & 14 & 120 & 25 & 0.007 & 1 \\
Sub. ev. rainfor. & 0.19 & 0.5 & 50 & 150 & 14 & 120 & 25 & 0.005 & 0.83 \\
Temp./subpolar ev. rainfor. & 0.39 & 0.5 & 65 & 250 & 14 & 120 & 25 & 0.007 & 0.5 \\
Temp. ev. seas. brl. for., sum. rain & 0.81 & 0.5 & 55 & 250 & 14 & 120 & 25 & 0.008 & 1 \\
Ev. brl. scler. for., winter rain & 0.47 & 0.5 & 55 & 250 & 14 & 120 & 25 & 0.008 & 1 \\
Trop./sub. ev. needle forest & 0.49 & 0.5 & 60 & 150 & 14 & 120 & 25 & 0.007 & 0.67 \\
Temp./subpolar ev. needle forest & 9.29 & 0.5 & 70 & 300 & 14 & 120 & 25 & 0.01 & 0.5 \\
Trop./sub. drought decid. for. & 2.88 & 0.5 & 50 & 150 & 14 & 120 & 25 & 0.007 & 1 \\
Cold-decid. for., with evergreens & 5.18 & 0.5 & 65 & 275 & 14 & 120 & 25 & 0.008 & 0.83 \\
Cold-decid. for., w/o evergreens & 3.99 & 0.5 & 55 & 250 & 14 & 120 & 25 & 0.007 & 1 \\
Xeromorphic for./woodland & 2.68 & 0.5 & 55 & 250 & 14 & 120 & 25 & 0.01 & 1 \\
Ev. brl. scler. woodland & 1.71 & 0.5 & 55 & 250 & 14 & 120 & 25 & 0.01 & 0.83 \\
Ev. needle woodland & 2.51 & 0.5 & 65 & 250 & 14 & 120 & 25 & 0.01 & 0.67 \\
Trop./sub. drought-decid. woodland & 3.7 & 0.5 & 50 & 150 & 14 & 120 & 25 & 0.01 & 1 \\
Cold-decid. woodland & 2.5 & 0.5 & 55 & 250 & 14 & 120 & 25 & 0.01 & 1 \\
Ev. brl. shrub/thicket, ev. dwarf shrub & 1.3 & 0.5 & 55 & 180 & 14 & 120 & 25 & 0.05 & 0.83 \\
Ev. needle/microphyllous shr./thick. & 0.67 & 0.5 & 65 & 180 & 14 & 120 & 25 & 0.05 & 0.67 \\
\hline
\end{tabular}

Continued on next page 
Table 3.1 Continued

Drought-decid. shrub/thicket

$\begin{array}{lll}0.83 & 0.5 & 60\end{array}$

$60 \quad 180$

$180 \quad 14$

Cold-decid. subalp./subpolar shrub

0.46

0.5

60

180

$14 \quad 120$

25

25

0.05

0.05

8.86

0.5

60

180

$14 \quad 120$

25

Arctic alpine tundra, mossy bog

7.34

250

14

120

25

$0.05 \quad 1$

Tall/med./short grass; $10-40 \%$ tree

6.46

250

Tall/med./short grass; $<10 \%$ tree

3.66

60

12

$80 \quad 50$

$0.01 \quad 0.5$

Tall/med./short grass; w/ shrub cov.

9.34

$-$

$60 \quad 20$

$0.01 \quad 0.33$

Tall grass; no woody cover

Med. grass; no woody cover

$0.81 \quad 0 \quad 50$

$0.78 \quad 0 \quad 50$

$\begin{array}{lll}6.1 & 0 & 50\end{array}$

$0.28 \quad 0 \quad 50$

Forb formations

17.56

$-$

-

11
10
10
10
10
10
10
-

10
10
10
10
10
10
-

$\begin{array}{ll}60 & 20 \\ 60 & 20\end{array}$

$\begin{array}{ll}- & 0.33 \\ - & 0.33\end{array}$

$\begin{array}{llll}60 & 20 & - & 0.33\end{array}$

$\begin{array}{llll}60 & 20 & - & 0.5\end{array}$

$\begin{array}{cccc}60 & 20 & - & 1 \\ 60 & 20 & - & 1 \\ - & - & - & -5\end{array}$




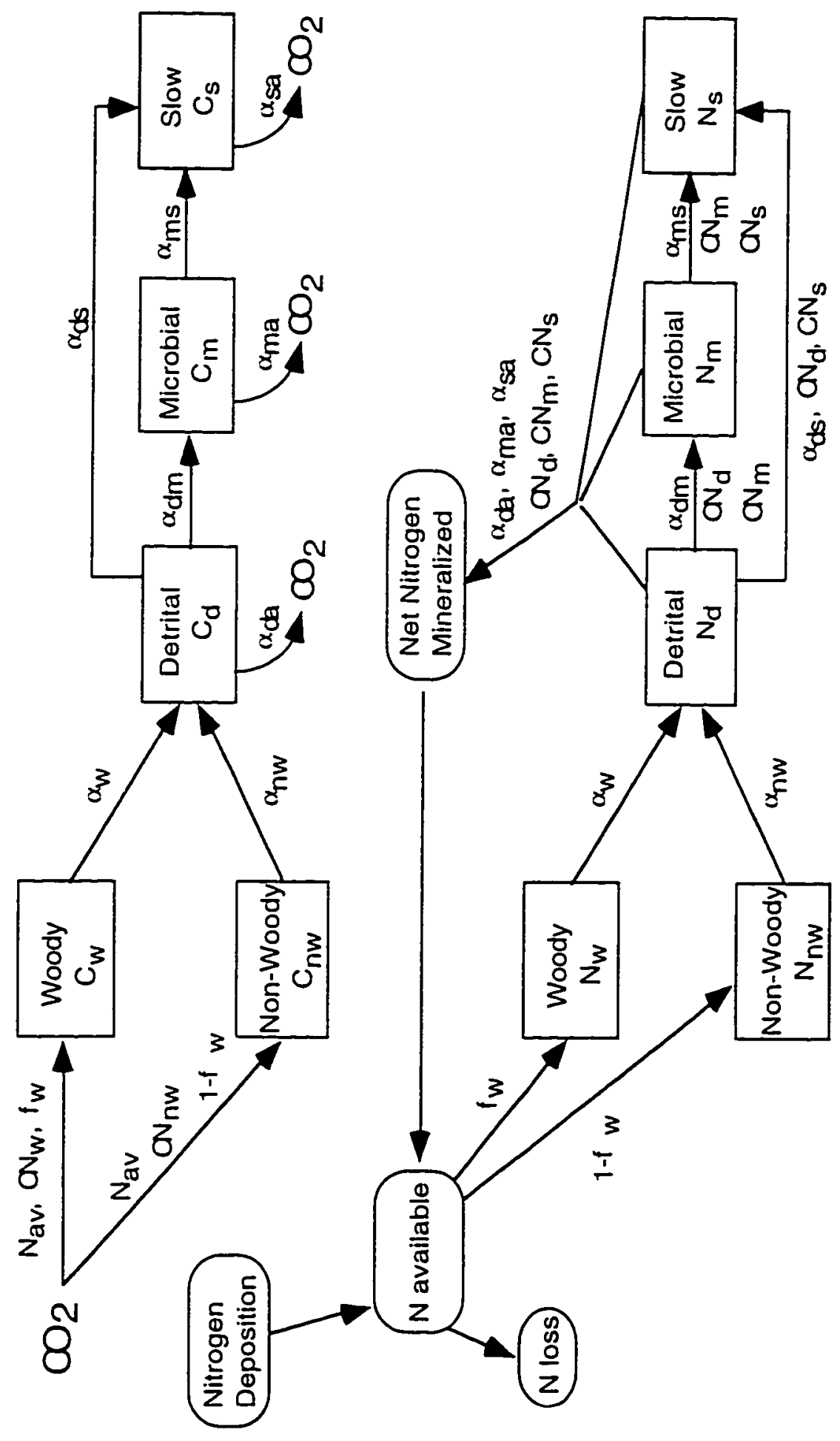

Figure 3.1 Carbon and nitrogen pools and fluxes in the NDEP model. Refer to text for description of symbols. 
where $\alpha_{w}$ and $\alpha_{n w}$ represent yearly litterfall fractions from woody and non-woody vegetation, respectively, and are equal to the inverse of the residence time. Litterfall from each pool enters the detrital $C$ pool $\left(C_{d}\right)$, which changes according to:

$$
\frac{d C_{d}}{d t}=\alpha_{w} \cdot C_{w}+\alpha_{n w} \cdot C_{n w}-\left(\alpha_{d a}+\alpha_{d m}+\alpha_{d s}\right) \cdot C_{d}
$$

where $\alpha_{d m}, \alpha_{d s}$ and $\alpha_{d a}$, are transfer coefficients from the detrital pool to the microbial pool, the slow pool, and to the atmosphere (respiration). The values depend on lignin content and mean annual temperature, and are taken from a global analysis of soil organic matter turnover times (Schimel et al. 1994). Changes in microbial $\left(C_{m}\right)$ and slow $\left(C_{s}\right)$ carbon pools are calculated from:

$$
\begin{aligned}
& \frac{d C_{m}}{d t}=\alpha_{d m} \cdot C_{d}-\left(\alpha_{m a}+\alpha_{m s}\right) \cdot C_{m} \\
& \frac{d C_{s}}{d t}=\alpha_{d s} \cdot C_{d}+\alpha_{m s} \cdot C_{m}-\alpha_{s a} \cdot C_{s},
\end{aligned}
$$

where again the $\alpha$ values are from Schimel et al. (1994).

Changes in woody $\left(N_{w}\right)$, non-woody $\left(N_{m w}\right)$, microbial $\left(N_{m}\right)$ and slow $\left(N_{s}\right)$ nitrogen pools are the same as those for carbon, divided by the $\mathrm{C}: \mathrm{N}$ ratios of the pool:

$$
\frac{d N_{i}}{d t}=\frac{d C_{i} / d t}{C N_{i}}
$$


but changes in detrital nitrogen $\left(N_{d}\right)$ depend on the $\mathrm{C}: \mathrm{N}$ ratios of both vegetation and detrital pools:

$$
\frac{d N_{d}}{d t}=\frac{\left(\alpha_{w} \cdot C_{w}\right)}{C N_{w}}+\frac{\left(\alpha_{n w} \cdot C_{n w}\right)}{C N_{n w}}-\frac{\left(\alpha_{d a}+\alpha_{d m}+\alpha_{d s}\right)}{C N_{d}}
$$

Net nitrogen mineralization $\left(N_{\min }\right)$ depends on the carbon transfer and the $\mathrm{C}: \mathrm{N}$ ratios of all three soil pools. Enough $\mathrm{N}$ to satisfy the $\mathrm{C}: \mathrm{N}$ ratio of each pool is "immobilized" during each transfer, with the remainder contributing to net $N_{\text {min }}$ :

$$
\begin{gathered}
N_{m u n}=\left[\frac{\left(\alpha_{d a}+\alpha_{d m}+\alpha_{d s}\right) \cdot C_{d}}{C N_{d}}+\frac{\left(\alpha_{m a}+\alpha_{m s}\right) \cdot C_{m}}{C N_{m}}+\frac{\alpha_{s a} \cdot C_{s}}{C N_{s}}\right] \\
-\left[\frac{\alpha_{d m} \cdot C_{d}}{C N_{m}}+\frac{\alpha_{d s} \cdot C_{d}}{C N_{s}}+\frac{\alpha_{m s} \cdot C_{m}}{C N_{s}}\right],
\end{gathered}
$$

and available nitrogen for plant uptake $\left(N_{a v}\right)$ is then calculated as the current year's deposition plus net $\mathrm{N}$ mineralization minus the rate of $\mathrm{N}$ loss:

$$
N_{a v}=N_{\text {dep }}+N_{\min }-N_{\text {loss }}
$$

$N_{\text {loss }}$ was set at $20 \%$ of $N_{\text {dep }}+N_{\min }$ for most of the simulations; this value is an approximate mean from a number of nitrogen budget srudies in temperate systems (e.g., Likens et al. 1981, Johnson et al. 1991). The sensitivity of the overall results to variations in $N_{\text {loss }}$ is discussed in the following section. 
Finally, the total carbon sink ( $C_{\text {sink }}$ ) due to $\mathrm{N}$ deposition at any time $t$ is equal to the sum of the changes in each of the five carbon pools:

$$
\frac{d C_{\text {sink }}}{d t}=\frac{d}{d t}\left(C_{w}+C_{n w}+C_{d}+C_{m}+C_{s}\right)
$$

because the accumulation of carbon in each pool (set initially to zero) is associated with a perturbation flux of anthropogenic $\mathrm{N}$ deposition.

\subsubsection{Results and discussion}

Each simulation of this perturbation model was run for 145 years (1845-1990). Our initial "best estimate" assumed $20 \%$ of available $\mathrm{N}$ was lost from the system and that $50 \%$ of $\mathrm{C}$ fixed in forested regions was allocated to woody tissue, giving a 1990 estimate for carbon uptake of $0.64 \mathrm{Pg} \mathrm{yr}^{-1}$, and a cumulative storage of $23.7 \mathrm{Pg}$. This simulation also showed that carbon storage in wood dominated the total sink (Fig. 3.2) due to high $\mathrm{C}: \mathrm{N}$ ratios in woody biomass coupled with turnover times that can exceed 100 years (Table 3.1). Despite long turnover times and high carbon contents for soil organic matter, our estimates of $\mathrm{C}$ storage in soils were relatively small (Fig. 3.2). This is not only because of narrower $\mathrm{C}: \mathrm{N}$ ratios in soils than in wood, but also because only a small fraction of the photosynthetically fixed carbon enters the large, more recalcitrant pools of soil organic matter (Townsend et al. 1995).

Our results support the existence of a sizable biospheric sink in northern temperate regions of the globe. Figure 3.3 shows that nearly all of the 1990 global sink occurs between about $25^{\circ}$ and $55^{\circ}$ north, and its latitudinal pattern is very similar to those shown by several inverse model estimates of carbon exchange driven by data on both $\mathrm{CO}_{2}$ and ${ }^{13} \mathrm{CO}_{2}$ (Tans et al. 


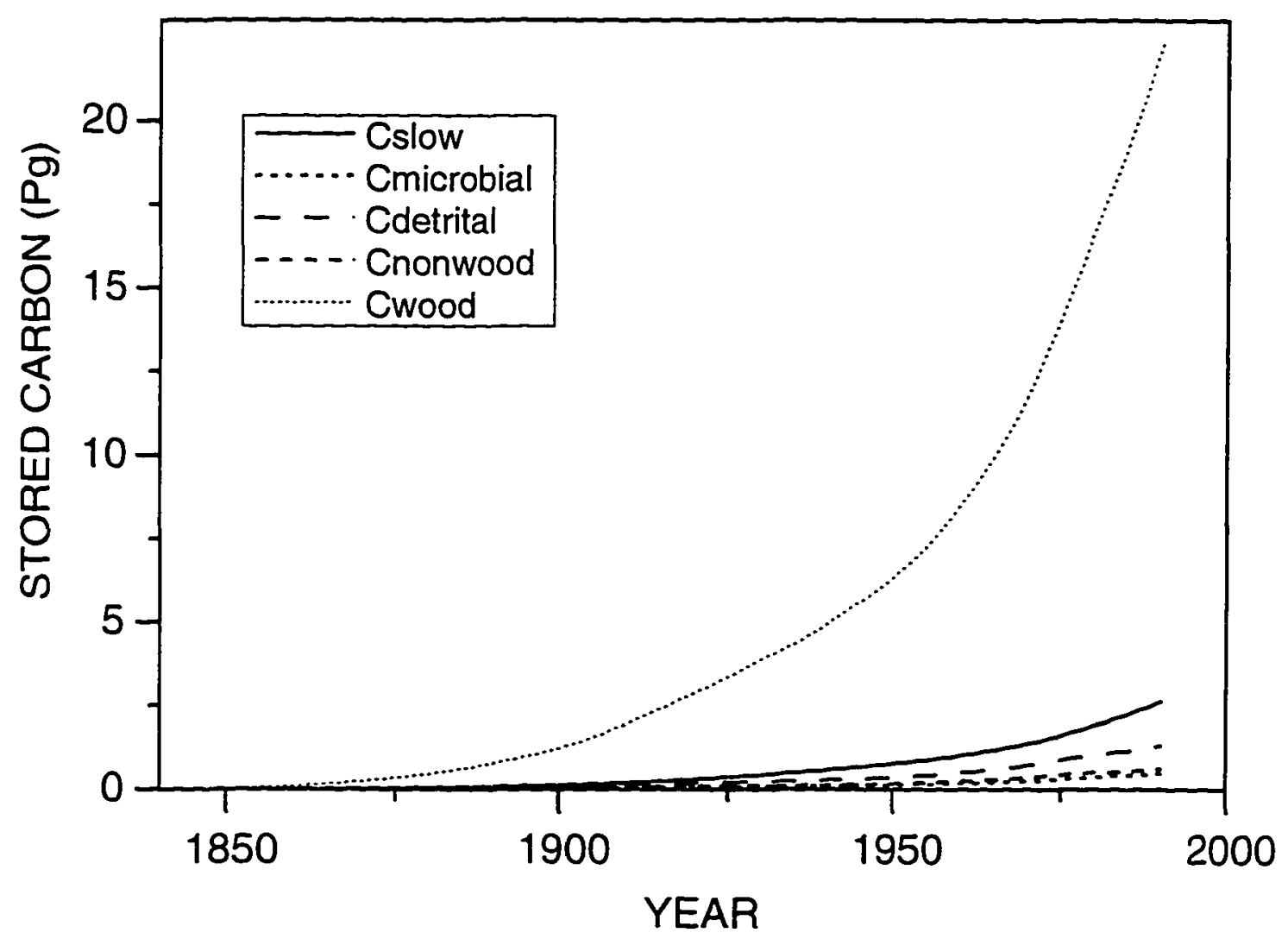

Figure 3.2 Stored carbon resulting from deposition of fossil fuel derived $\mathrm{N}$ as a function of time for woody, non-woody, detrital, microbial, and slow carbon pools. Simulation assumed that $50 \%$ of $C$ fixed in forested regions was allocated to wood $\left(f_{w}=0.5\right)$, and that $20 \%$ of soil inorganic $\mathrm{N}$ was lost from the system each year $\left(N_{\text {loss }}=0.2\right)$. 


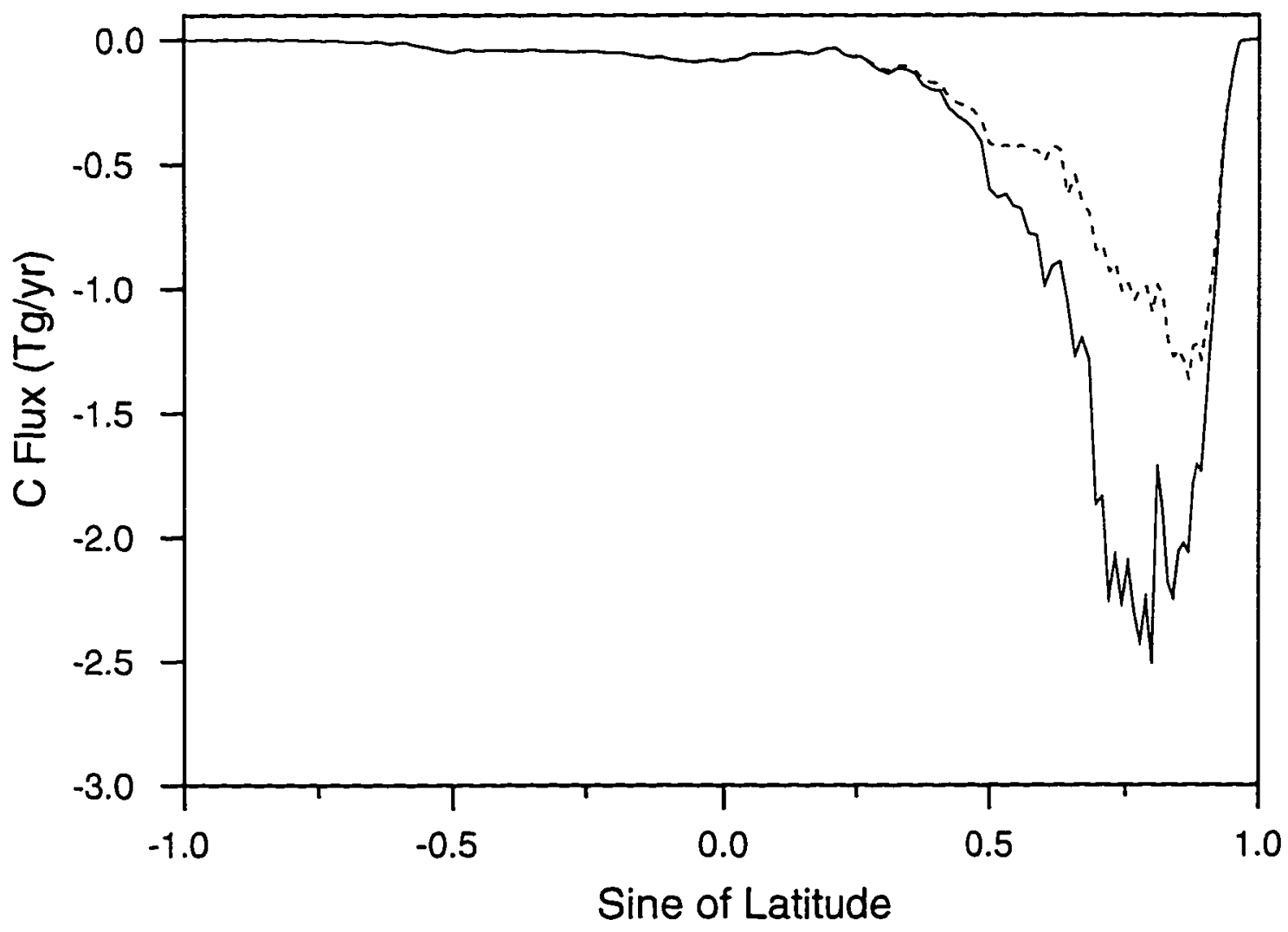

Figure 3.3 Latitudinal distribution of the net carbon flux between land and atmosphere for 1990 in $\mathrm{Tg} \mathrm{yr}^{-1}$. The solid line is from the simulation with $f_{w}=0.5$ and $N_{\text {loss }}=0.2$, where 1990 global C uptake was $0.64 \mathrm{Pg}$; the dotted line is for the scenario in which $N_{\text {loss }}$ increases with cumulative $N_{\text {dep }}$, where 1990 uptake was $0.39 \mathrm{Pg} \mathrm{yr}^{-1}$. Negative flux values indicate a terrestrial sink, and negative values for the sine of latitude are the Southern Hemisphere. 
1989, Enting and Mansbridge 1991, Ciais et al. 1995a). The $1^{\circ} \times 1^{\circ}$ patterns of $\mathrm{C}$ uptake show that the strongest sinks are in three distinct mid-latitude regions: the eastern US, Europe, and eastern Asia (Fig. 3.4a). Not surprisingly, these regions also have high $\mathrm{NO}_{\mathrm{y}}$ deposition (Fig. 3.4b). However, vegetation type has a profound effect on the amount of $\mathrm{C}$ stored per unit $\mathrm{N}$. The largest sinks are in the forested regions, where significant amounts of $\mathrm{N}$ are incorporated into woody tissue at high $\mathrm{C}: \mathrm{N}$ ratios, but much of the area experiencing high $\mathrm{N}$ deposition is covered by grasslands or cultivated areas. The lack of wood in grassland biomes severely limits the ability of $\mathrm{N}$ to stimulate carbon sequestration, and we have assumed that no storage occurs in cultivated regions.

Because storage as wood dominates the total sink, the fraction of excess $C$ uptake which is allocated to woody biomass has a substantial effect on total carbon storage in our analysis. We note that allocation can vary with vegetation and soil type, nutrient status, and stand age, but in most forests, sizable fractions of photosynthate appear to be allocated to both woody and non-woody components (Aber and Melillo 1991). To evaluate the importance of allocation to our estimates, we ran a series simulations in which the percent of fixed $C$ which was allocated to wood was varied from 10 to $100 \%$. Corresponding estimates of net global C uptake for 1990 varied from 0.23 to $1.15 \mathrm{Pg} \mathrm{yr}^{-1}$, respectively, and the cumulative sink varied from 8.7 to $41.8 \mathrm{Pg}$ (Fig. 3.5).

Allocation may also change over time as the availability of $\mathrm{N}$ changes, as may the $\mathrm{C}: \mathrm{N}$ ratios of the plant tissue (Boring et al. 1988, Vitousek et al. 1988). These simulations suggest that net $C$ uptake is likely to be more sensitive to any changes in allocation than to $C: N$ ratio changes within each tissue type. This is because a change in allocation represents a substantial change in both the amount of $\mathrm{C}$ fixed per unit $\mathrm{N}$ and in the overall terrestrial lifetime of the carbon fixed. Changes in $\mathrm{C}: \mathrm{N}$ ratio within a tissue type due to increasing $\mathrm{N}$ availability will 
never be as large as the difference in $\mathrm{C}: \mathrm{N}$ ratios between woody and foliar components.

Furthermore, while changes in $\mathrm{C}: \mathrm{N}$ ratio can affect the rate at which the corresponding litter is decomposed, such changes are small relative to the difference in turnover times of woody versus foliar litter (Schimel et al. 1994).

Our $1990 \mathrm{C}$ sink estimate of $0.64 \mathrm{Pg} \mathrm{yr}^{-1}$ assumed that only $20 \%$ of $\mathrm{N}$ inputs were lost from all biomes. This value is an approximate mean of values reported from several studies in temperate forested watersheds such as Hubbard Brook and Walker Branch (Likens et al. 1981, Johnson et al. 1991). It is also consistent with a large scale analysis of regional $\mathrm{N}$ inputs and river losses in the major river basins draining into the North Atlantic Ocean (Howarth et al. In press). If, as is generally assumed, the temperate regions of the globe are primarily $\mathrm{N}$ limited, it makes sense that retention of $\mathrm{N}$ inputs should be high. However, retention of deposited $\mathrm{N}$ is known to vary with vegetation type, forest age, the form of deposition (wet vs. dry), the cation exchange capacity of the soil, and the amount of sulfur as well as $\mathrm{N}$ deposition (Vitousek et al. 1988, Schulze et al. 1989, Johnson 1992). In areas of chronically high deposition, $\mathrm{N}$ losses may actually exceed inputs (Schulze et al. 1989), and in general, a steady increase in $\mathrm{N}$ loading should cause a decrease in overall retention as the systems become "nitrogen saturated" (Aber et al. 1989).

We explored how sensitive our calculated sink is to the fraction of $\mathrm{N}$ lost by scaling $\mathrm{N}$ losses to total $\mathrm{NO}_{\mathrm{y}}$ deposited. This has the effect of increasing $N_{\text {loss }}$ with time, and is a simple way to incorporate gradual $\mathrm{N}$ saturation of high deposition areas into the model. By assuming a linear increase in losses ( $N_{\text {loss }}$ ) from $20 \%$ of deposition inputs at the lowest deposition value in the entire simulation to $100 \%$ at the highest, we cut the "best" estimate for 1990 by about $40 \%$, giving a global sink of $0.39 \mathrm{Pg} \mathrm{C} \mathrm{yr}{ }^{-1}$, and cut the cumulative $\mathrm{C}$ uptake from $23.7 \mathrm{Pg}$ to 18.5 $\mathrm{Pg}$ (Fig. 3.5). Because the effect of increasing $N_{\text {loss }}$ is greatest in the highest deposition regions. 
a)

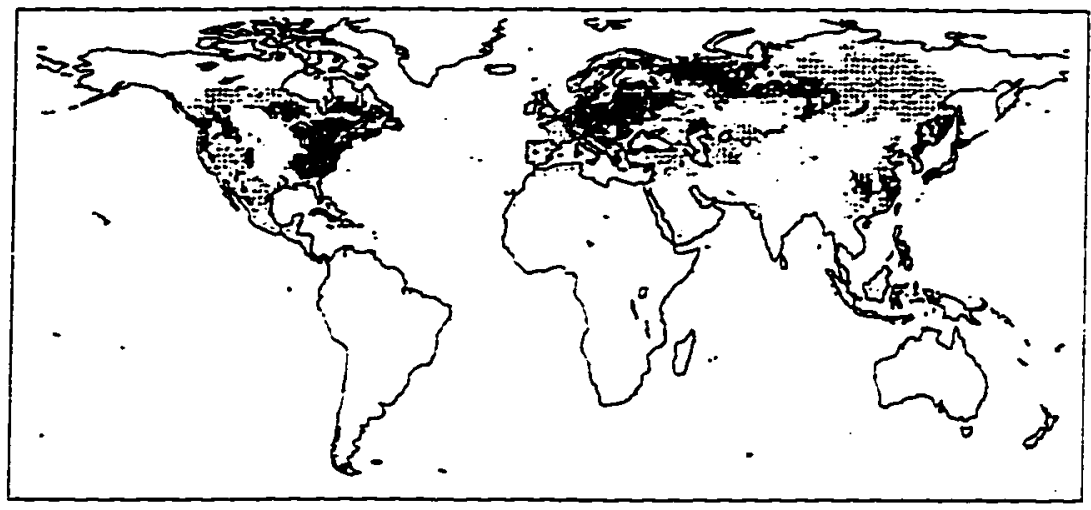

$\mathrm{gCm} \mathrm{m}^{-2}$

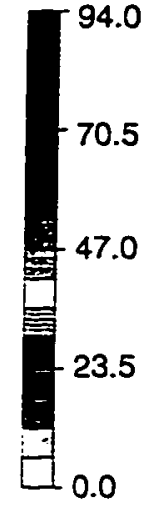

b)

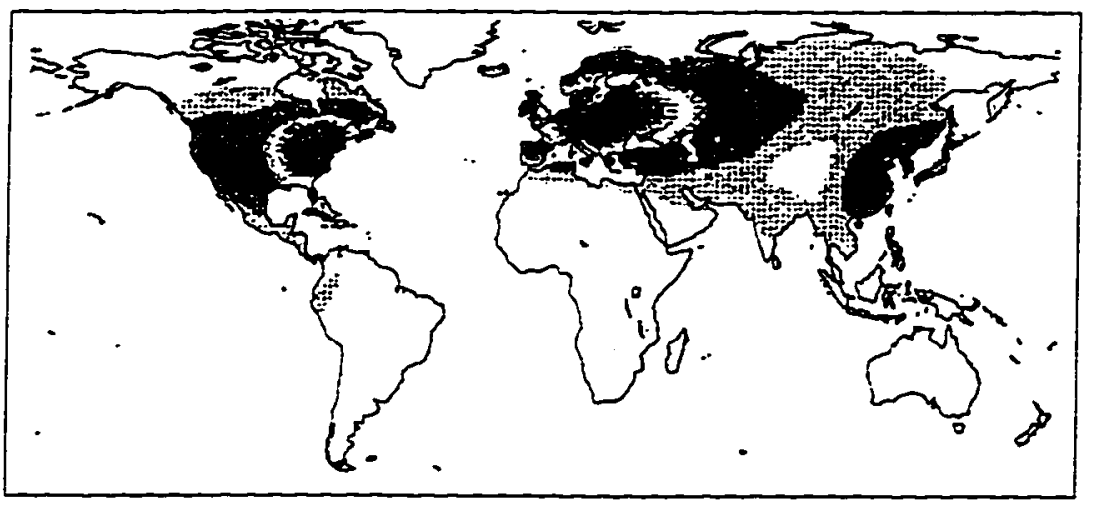

$\mathrm{kgN} \mathrm{km}{ }^{-2}$

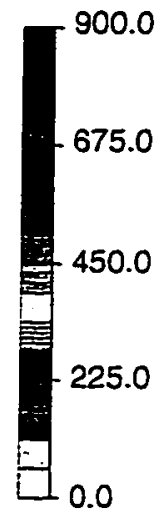

c)

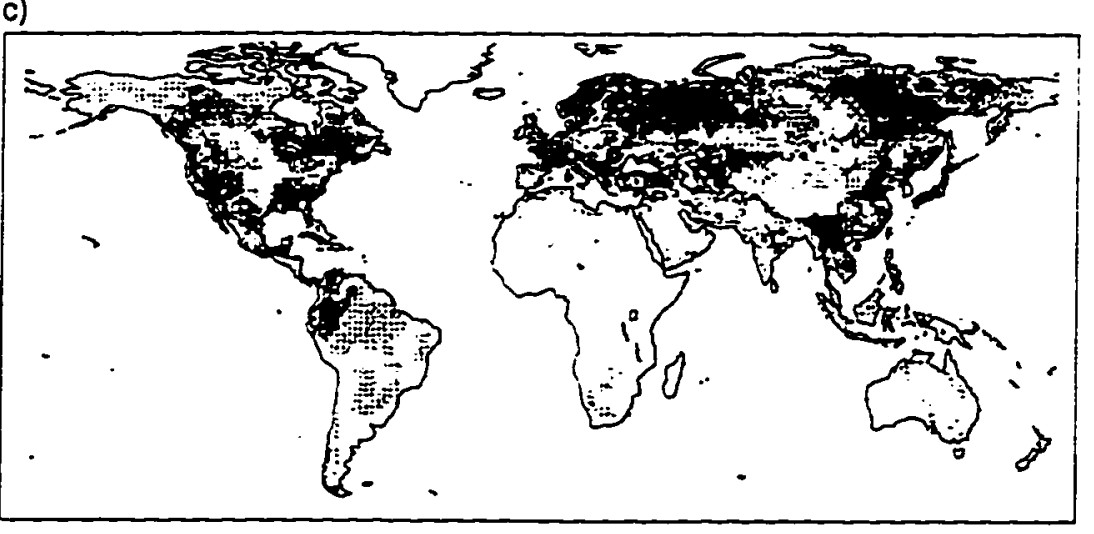

$\mathrm{gC} \mathrm{m}^{-2}$

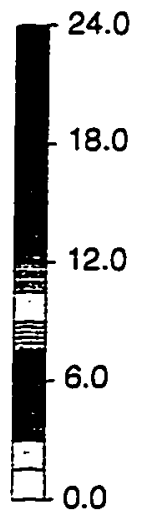

Figure 3.4 (a) Spatial distribution of the 1990 carbon sink resulting from fossil fuel $N$ deposition between 1845 and 1990. Values are in $\mathrm{g} \mathrm{C} \mathrm{m}^{-2}$; the simulation used $f_{w}=0.5$ and $N_{\text {loss }}=0.2$, where total global $C$ uptake was $0.64 \mathrm{Pg}$. (a) Fossil fuel nitrogen deposition on land $\left(\mathrm{kg} \mathrm{km}^{-2}\right)$ for 1990 as estimated by the GRANTOUR atmospheric transport model (Penner et al. 1991). (c) Same as (a) except that for this simulation, $N_{\text {loss }}$ increases with $N$ deposition. 


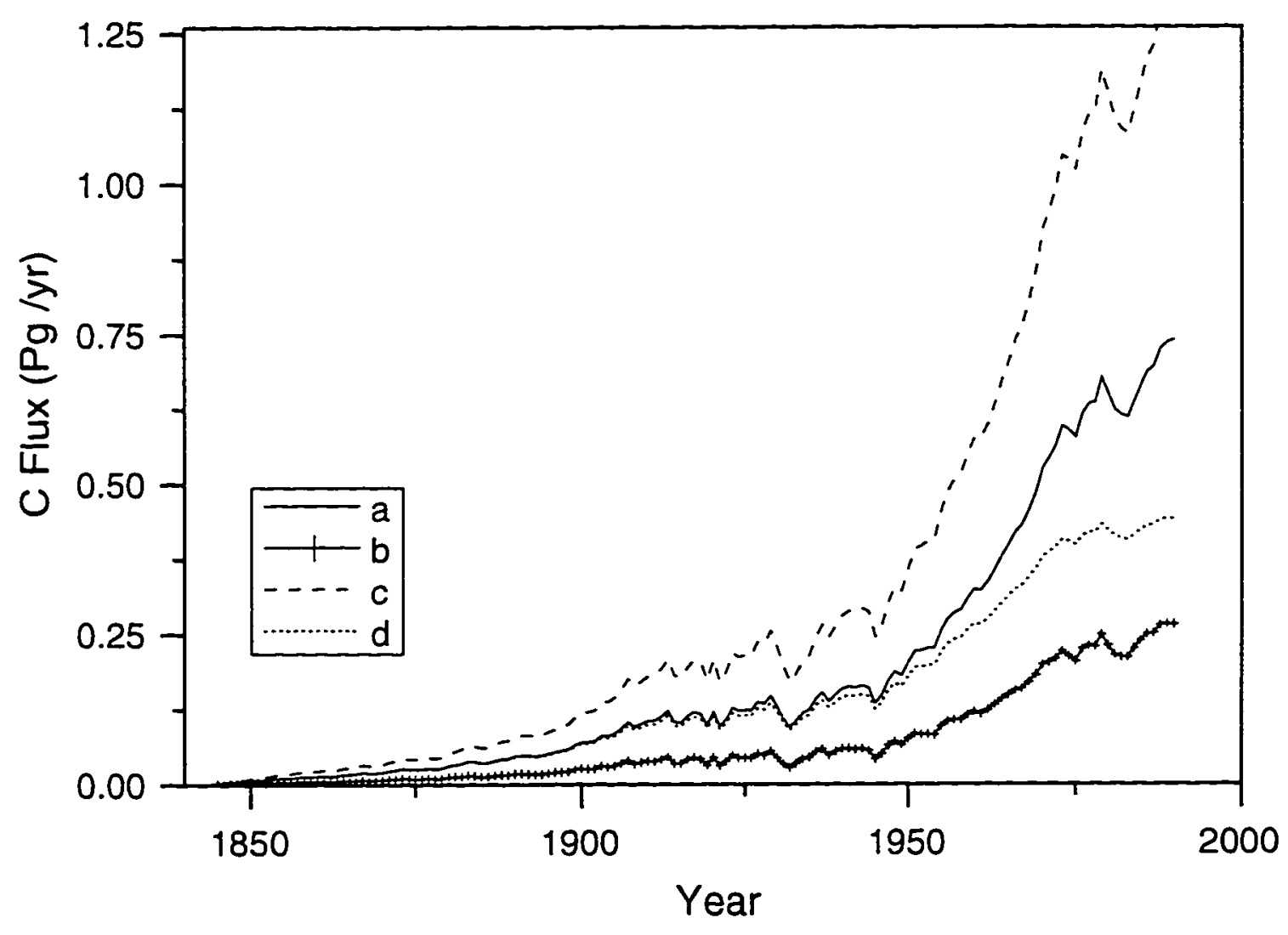

Figure 3.5 Sensitivity of the estimated carbon sink to the fraction of carbon allocated to wood $\left(f_{w}\right)$, and to the amount of $\mathrm{N}$ lost from the system: (a) $f_{w}=0.5, N_{\text {loss }}=0.2 ;$ (b) $f_{w}=0.1$, $N_{\text {loss }}=0.2$; (c) $f_{w}=1.0, N_{\text {loss }}=0.2$; (d) $f_{w^{\prime}}=0.5, N_{\text {loss }}$ varies from $20 \%$ of deposition at the lowest deposition value to $100 \%$ at the highest. 
the spatial distribution of $1990 \mathrm{C}$ uptake also changed dramatically (Fig. 3.4c). Forests in the highest deposition regions no longer completely dominate the sink, and the spatial variability in lower deposition regions is more easily seen.

We believe 0.39-0.64 $\mathrm{Pg} \mathrm{yr}^{-1}$ (for 1990) and 18.5-27.3 $\mathrm{Pg}$ (cumulatively since 1845) are the most reasonable ranges for the global carbon sink resulting from deposition of fossil fuel-derived nitrogen. By accounting for net uptake of $\mathrm{C}$ along with spatial variation in biome types and $\mathrm{N}$ deposition, we arrived at an estimate that is between those of Schindler and Bayley (1993) and Peterson and Melillo (1985). Recent estimates for the total "missing carbon sink" are around $1.5-2 \mathrm{Pg} \mathrm{yr}^{-1}$ for the $1980 \mathrm{~s}$ and between $50-125 \mathrm{Pg}$ cumulatively since the late 18 th century (Moore and Braswell 1994. Schimel et al. 1995; Chapter 2). Our estimate therefore represents about $25 \%$ of this sink, either for the single year of 1990 or cumulatively since 1845. It should be remembered, however, that significant additional $C$ uptake may occur from deposition of $\mathrm{NH}_{\mathbf{x}}$ (see Section 3.3), and that the magnitude of our estimates are also dependent on the veracity of the Matthews (1983) data base for vegetation distribution. Finally, it is worth noting that while analyses of the residual sink often focus upon a single factor, three of the most commonly proposed mechanisms for terrestrial storage of excess $\mathrm{CO}_{2}\left(\mathrm{~N}\right.$ deposition, $\mathrm{CO}_{2}$ fertilization, and forest regrowth) are not independent. For example, $\mathrm{N}$ deposition may accelerate rates of forest regrowth, and may enhance the effects of $\mathrm{CO}_{2}$ fertilization, as nutrient limitation appears to constrain plant response to elevated $\mathrm{CO}_{2}$ (Comins and McMurtrie 1993).

Fossil-fuel $\mathrm{N}$ may stimulate biospheric storage of fossil-fuel $\mathrm{CO}_{2}$, but $\mathrm{N}$ deposition should not be viewed as a purely "beneficial" phenomenon. The potentially harmful effects of acid rain due to emissions of nitrogen and sulfur oxides are well documented (Schulze 1989. Johnson et al. 1991). Chameides et al. (1994) estimate that increasing $\mathrm{NO}_{\mathrm{x}}$ emissions will cause a threefold increase in plant exposure to tropospheric ozone by early next century in some mid- 
latitude regions. Finally, deposition of fossil-fuel $\mathrm{N}$ is likely to double in less than thirty years (Galloway et al. 1994), but continued exposure to increasing nitrogen inputs should increase nitrogen losses, and may prove harmful, resulting in ecosystem decline and an overall reduction in net carbon uptake (Aber et al. 1989; see Section 3.4). Even if the excess $\mathrm{N}$ does not cause ecosystem decline, the global increase in available $\mathrm{N}$ must be causing a decrease in $\mathrm{N}$ limitation of terrestrial ecosystems, which would decrease responses to additional $\mathrm{N}$. The persistence of an $\mathrm{N}$-derived carbon sink will depend on the balance between a probable decrease in excess carbon uptake in many temperate forests that have experienced substantial $\mathrm{N}$ loading, and the response of previously low deposition regions that will experience rapid increases in $\mathbf{N}$ inputs over the next few decades. The latter changes will be primarily tropical, therefore global carbon storage due to $\mathrm{N}$ deposition may be saturating now.

The response of terrestrial ecosystems to any perturbation will depend on their nutrient status and stand age, which in turn are dependent on the history of both natural and anthropogenic disturbance (Aber et al. In press; Chapter 4). For this reason, the assumption of ecosystem steady-state perturbed only by $\mathrm{N}$ deposition (in the NDEP formalism) is not globally applicable, but the extent to which this is the case is not known. The highly variable patterns of land use/cover have been mapped at various scales, but are difficult to project either backward or forward in time because they are driven by complex local-to-global scale social and economic pressures. As a first step, we have used a static map of land use/cover for this analysis, but future studies will benefit from a simplified representation of historical regionalscale land conversion (e.g., Esser 1989) and natural regeneration processes (e.g., fire) (Aber and Melillo 1991) combined with rules relating allocation and the degree of $\mathrm{N}$-limitation to ecosystem type and age. These rules likely can be developed from field observations (e.g., 
Stoddard 1994, Schulze et al. 1989) and process-based models such as Century (Parton et al. 1987) and PnET (Aber and Federer 1992, Aber et al. In press).

\subsection{The Spatial Distribution of Atmospheric Nitrogen Deposition and its Impact on Carbon Uptake by Terrestrial Ecosystems ${ }^{\dagger}$}

\subsubsection{Introduction}

In a previous study we used NDEP, a perturbation model, to quantify the terrestrial carbon storage due to human-induced nitrogen deposition (Townsend et al. 1996; Section 3.2). We showed that enhanced carbon uptake is sensitive to (1) the details of biogeochemical cycling, (2) the spatial distribution of land and vegetation cover, and (3) the spatial distribution and quantity of deposited nitrogen. In this section, we examine the importance of the latter, focusing on the role of atmospheric chemistry and transport models (CTMs). The representation of physical and chemical processes, and thus the simulation of deposition, can differ significantly among the extant models. These differences propagate into uncertainties in the estimated $\mathrm{N}$-induced $\mathrm{C}$ sink. Further, atmospheric pollutants that tend to relieve ecosystem $\mathrm{N}$-limitation and eliminate the carbon sink potential of ecosystems (most notably ozone) are also simulated by CTMs. Thus, accurate representation of the chemistry, transport, and deposition of $\mathrm{NO}_{y}, \mathrm{NH}_{x}$, and $\mathrm{O}_{3}$ is important for understanding the bio-atmospheric coupling of the carbon and nitrogen cycles.

The deposited nitrogen that is of interest in this study is that $\mathrm{N}$ derived from both industrialization and the expansion of urban and agricultural complexes (Chameides et al. 1994), essentially the additional $\mathrm{N}$ which represents a perturbation to the pre-industrial nitrogen cycle. Patterns of $\mathrm{N}$ deposition are not extremely well-known because there is no globally-

\footnotetext{
${ }^{\dagger}$ Adapted from the article by E.A. Holland, B.H. Braswell, J.-F. Lamarque, J. Sulzman, J.-F. Müller, F. Dentener, G. Brasseur, H. Levy II, J.E. Penner, and G.-J. Roelofs, Journal of Geophysical Research, In press. Copyright by the American Geophysical Union.
} 
distributed sampling of either wet or dry nitrogen deposition. Measurements of precipitation inputs of many cations and anions (including $\mathrm{NH}_{4}{ }^{+}$and $\mathrm{NO}_{3}$ ) have been made in country-bycountry networks established to quantify acid precipitation, but these measurements provide partial coverage of the globe, and generally do not include all the nitrogen compounds deposited. Measurements of dry deposition are particularly sparse (US Environmental Protection Agency). Thus, three dimensional chemical transport models provide the only globally distributed information on the deposition of nitrogen, allowing the separation of the nitrogen into that attributable to background processes and to the intensification of human activity. Such information can be used as input into our perturbation model (NDEP).

\subsubsection{Approach}

We use the simulated deposition of five different CTMs to represent variation in the spatial distribution of deposited nitrogen: ECHAM (Roelofs and Lelieveld 1995); GCTM (Levy et al. In press b); GRANTOUR (Penner et al. 1991,1994); IMAGES (Müller 1992, Müller and Brasseur 1995); and MOGUNTIA (Crutzen and Zimmerman 1991, Dentener and Crutzen 1993,1994, Zimmerman et al. 1989). The models differ in the source magnitudes and distributions of $\mathrm{NO}_{\mathrm{x}}$ used, in their chemical transformation schemes, and in the way compounds are transported. An abbreviated summary of the differences amongst the models is provided in the text below (for an exhaustive comparison, see Holland et al. In press). All the models include $\mathrm{NO}_{x}$ emissions, chemistry, and transport. However, only one model, MOGUNTIA, represents $\mathrm{NH}_{\mathrm{x}}$ emissions and their subsequent fate. Thus model-to-model comparisons of $\mathrm{NO}_{\mathrm{y}}$ deposition consider only the fate of emitted $\mathrm{NO}_{\mathbf{x}}$, which likely underestimates total $\mathrm{N}$ deposition by more than $100 \%$ because $\mathrm{NO}_{\mathrm{y}}$ comprises $\sim 1 / 2$ of total wet and dry $\mathrm{N}$ deposition (Dentener 
and Crutzen 1994). Therefore, our most comprehensive estimate of increased carbon storage due to $\mathrm{N}$ deposition is made using both $\mathrm{NH}_{\mathrm{x}}$ and $\mathrm{NO}_{\mathrm{y}}$ deposition estimates.

The influence of nitrogen deposition cannot be accurately described by examining only the $\mathrm{N}$ that is deposited in a single year (e.g., Peterson and Melillo 1985, Schindler and Bayley 1993) because $N$ is continually recycled in terrestrial ecosystems by the release or mineralization of $\mathrm{N}$ during decomposition and subsequent plant and microbial uptake of that $\mathrm{N}$. Therefore, in the NDEP model, we calculate the cumulative effect of $\mathrm{N}$ deposition by estimating the amount deposited over the last century. beginning with 1860 . The nitrogen and fixed carbon as a result of that deposition then cycles and the nitrogen is recirculated within the ecosystem allowing for losses due to leaching and gaseous emissions. All estimates of carbon storage are thus the cumulative result of nitrogen deposition and differ substantially from other “instantaneous" estimates.

\subsubsection{Description and comparison of 3-D chemical transport models}

All the CTMs included in this study incorporate the same basic elements: sources of $\mathrm{NO}_{\mathrm{x}}$ (or $\mathrm{NH}_{\mathrm{x}}$ ) are released on a latitude by longitude grid and transported. The compounds undergo chemical transformation, are deposited back to the surface, or are released to the stratosphere. The primary mechanisms for removal are by precipitation and dry deposition. The details of how the processes are represented, the space and time resolution used, and the emphasis placed on different components differ substantially amongst the models; some of the major differences, and the resultant differences in their predictions of $\mathrm{N}$ deposition will be discussed below.

The spatial and temporal resolution of the five models differ from one another. GCTM has the finest horizontal resolution (though still coarse by ecological standards) with $2.4^{\circ} \times 2.4^{\circ}$ 
grid cells and MOGUNTIA has the coarsest horizontal resolution with $10^{\circ} \times 10^{\circ}$ grid cells. The height of the atmosphere represented varies between 16 and $32 \mathrm{~km}$, and the number of vertical layers in the model atmosphere varies between 10 and 25 . IMAGES uses the most highly resolved vertical layering and MOGUNTIA the coarsest. Each model is elaborate in a different aspect: for example, GCTM has the most refined horizontal and temporal resolution, while IMAGES has the most complex representation of chemistry.

The transport schemes used by the various models differ in the climatologies which drive them and in their transport schemes. ECHAM is a general circulation model implemented such that chemistry and transport are interactive (i.e., the chemistry model is run "on-line"). The transport time of the models ranges from 26 minutes for GCTM to 1 day for IMAGES. Two of the models, IMAGES and MOGUNTIA, use global climatologies of wind and precipitation. All of the models represent diffusion. They differ in the amount of sub-grid mixing, the convective schemes used, and exchanges with the planetary boundary layer. ECHAM has the most sophisticated convective scheme with penetrative, shallow, and mid-level convection.

The complexity of the chemical schemes used also differs amongst the five models. IMAGES has the most sophisticated chemistry with 125 reactions (including 2 photolytic reactions) and 41 species (including 7 different hydrocarbons, oxygenated organics, PAN, MPAN, and $\mathrm{N}_{2} \mathrm{O}_{5}$ reactions on aerosols). GRANTOUR uses a simple chemical scheme including $\mathrm{HNO}_{3}, \mathrm{NO}$, and $\mathrm{NO}_{2}$, but no reactions with PAN or other organic species.

The spatial distribution of the deposited nitrogen depends on interactions of the transport and chemical schemes with both wet and dry deposition. Two of the models, ECHAM and GCTM, use the same dry deposition scheme, which includes effective wind speed. The other three models use essentially the same equation for dry deposition: 


$$
F=V_{d} \cdot n \cdot Y
$$

where $V_{d}$ is the deposition velocity $\left(\mathrm{cm} \mathrm{s}^{-1}\right), n$ is the particle density (mole $\mathrm{cm}^{-3}$ ), and $Y$ is the mixing ratio of the gas $\left(\mathrm{cm}^{3} \mathrm{~cm}^{-3}\right)$. Each model differs in the assigned deposition velocities and whether diurnal variations in deposition velocity are included. Dry deposition velocities for $\mathrm{NO}_{2}$ are consistent amongst the models but vary substantially for $\mathrm{NO}$ and $\mathrm{HNO}_{3}$. Dry deposition velocities over land are specific to vegetation type for IMAGES, but independent of vegetation type in the other models. Wet deposition depends on the rate of precipitation, but the details of the formulations vary, particularly the different types of precipitation represented and the solubility coefficients implemented for the different chemical species.

\subsubsection{Sources of NOx}

Total $\mathrm{NO}_{\mathrm{x}}$ sources for the five models range from 35 to $48.8 \mathrm{Tg} \mathrm{N} \mathrm{yr}^{-1}$ (Table 3.2). Variations in fossil fuel emissions are smaller (approximately 10\%) and primarily driven by the choice of reference year. The lowest fossil fuel emissions are from GCTM, which uses a reference year of 1985 (Benkovitz et al. In press); GRANTOUR, which has the highest emissions, uses 1990 as the reference year. The remainder of the variation amongst the $\mathrm{NO}_{\mathrm{x}}$ emission estimates are driven by differences in the natural sources: lightning, biomass burning, and soils. The variability in the soil source and part of the biomass burning source are largely due to differing representations of biological controls over $\mathrm{NO}_{\mathrm{x}}$ production, particularly the inclusion of canopy scavenging of $\mathrm{NO}_{x}$ emitted at the soil surface. Transport and oxidation of $\mathrm{N}_{2} \mathrm{O}$ from the stratosphere and aircraft emissions (reported aircraft emissions ranged from 0.23

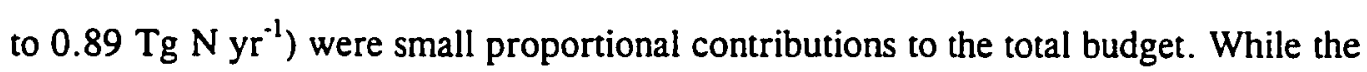


Table 3.2 $\mathrm{NO}_{\mathrm{x}}$ sources in $\mathrm{Tg} \mathrm{N} \mathrm{yr}^{-1}$ for five 3-D chemical transport models.

\begin{tabular}{|c|c|c|c|c|c|}
\hline $\mathrm{NO}_{\mathrm{x}}$ Emissions & ECHAM $^{\prime}$ & $\mathrm{GCTM}^{2}$ & GRANTOUR $^{3}$ & IMAGES $^{4}$ & MOGUNTIA $^{5}$ \\
\hline Fossil fuel & 20.0 & 21.0 & 22.4 & 21.9 & 20.0 \\
\hline Lightning & 4.0 & 3.0 & 10.0 & 8.0 & 5.0 \\
\hline Soils & 10.0 & 5.5 & 5.0 & 6.7 & 4.0 \\
\hline Aircraft & - & 0.45 & 0.23 & 0.44 & 0.6 \\
\hline $\begin{array}{l}\text { Biomass } \\
\text { burning }\end{array}$ & 6.0 & 8.5 & 10.0 & 4.4 & 6.0 \\
\hline Stratosphere & - & 0.64 & 0.2 & 0.2 & - \\
\hline Total & 40.0 & 38.6 & 48.8 & 42.6 & 35.0 \\
\hline $\begin{array}{l}{ }^{1} \text { Roelofs and Le } \\
{ }^{2} \text { Kasibhatla et al } \\
\text { press, Levy et a } \\
{ }^{3} \text { Penner et al. } 19 \\
{ }^{4} \text { Müller } 1992, N \\
{ }^{5} \text { Dentener and C }\end{array}$ & $\begin{array}{l}\text { eld } 1995 . \\
991,1993, \mathrm{~L} \\
\text { n press a,b. } \\
1994 .\end{array}$ & $\begin{array}{l}\text { et al. } 19 \\
1995 .\end{array}$ & Yienger and $L$ & 1995, Ben & itz et al. In \\
\hline
\end{tabular}

estimates of lightning production vary widely, lightning production of $\mathrm{NO}_{\mathrm{x}}$ is independent of human activity and so is not relevant to this perturbation study.

A large proportion of both the soil and biomass burning fluxes can be attributed to human activity. Among these five models, assumed biomass burning release of $\mathrm{NO}_{\mathrm{x}}$ varies by as much as $100 \%$ (ranging from $4.7-10 \mathrm{Tg} \mathrm{N} \mathrm{yr}^{-1}$ ), reflecting the high degree of uncertainty. Assumed total soil emissions of $\mathrm{NO}_{\mathrm{x}}$ also varied by more than $100 \%$ with roughly the same range (4.0-10 $\mathrm{Tg} \mathrm{N} \mathrm{yr} \mathrm{r}^{-1}$ ). A higher estimate of the global flux of $\mathrm{NO}_{\mathrm{x}}$ from soils, $20 \mathrm{Tg} \mathrm{N} \mathrm{yr}{ }^{-1}$, was made by Davidson (1991) based on a compilation of available estimates, while the 1994 IPCC estimate is $12 \mathrm{Tg} \mathrm{N} \mathrm{yr}^{-1}$ (Prather et al. 1995). Fertilizer application also substantially increases $\mathrm{NO}_{\mathrm{x}}$ fluxes from soils because as much as $10-20 \%$ of fertilizer $\mathrm{N}$ applied to tropical agricultural soils can be returned within one year. Thus fertilizer $\mathrm{N}$ could contribute substantially to global soil $\mathrm{NO}_{\mathrm{x}}$ emissions but only the IPCC (and GCTM) estimates incorporate this additional flux (Shepard et al. 1991, Yienger and Levy 1995). In GCTM, a much smaller 
percentage (2.5\%) of applied fertilizer is returned to the atmosphere as $\mathrm{NO}_{\mathrm{x}}$. The GCTM formulation is based on analysis of measurements made in mostly temperate ecosystems, and thus may be an underestimate of the fertilizer-induced $\mathrm{NO}_{\mathrm{x}}$ flux (Yienger and Levy 1995). IMAGES incorporates the influence of fertilizers indirectly by tripling the emissions associated with crops in developed countries (Müller 1992). It is possible that the total global soil flux may be underestimated in these models and that a substantial proportion of the global soil flux may be driven by human perturbation.

Vegetation scavenging of $\mathrm{NO}_{\mathbf{x}}$ may further contribute to some of the uncertainty amongst the estimates of soil $\mathrm{NO}_{\mathrm{x}}$ fluxes because vegetation can scavenge as much as $60-75 \%$ of the $\mathrm{NO}_{\mathrm{x}}$ emitted at the soil (Bakwin et al. 1990a,b, Jacob and Bakwin 1991, Yienger and Levy 1995). This effect is represented in MOGUNTIA and GCTM but not in ECHAM or GRANTOUR. A more simplistic vegetation scavenging of $\mathrm{NO}_{\mathrm{x}}$ is included in the dry deposition scheme for IMAGES. Much of the overall variation in sources between the models results from variations in the representation of the non-industrial sources of $\mathrm{NO}_{\mathbf{x}}$ (lightning, biomass burning, and soil emissions), many of which are influenced by human activity and so are reflected in each model's estimate of the carbon sink.

Because of the sensitivity of the carbon sink calculation to the quantity of $\mathrm{N}$ deposited as well as its spatial distribution (Townsend et al. 1996; Section 3.2), we first considered only fossil fuel-derived $\mathrm{NO}_{\mathrm{y}}$ (from stationary and aircraft sources) which is clearly a perturbation to the Earth system, and then considered a range of proportions of non-fossil fuel derived $\mathrm{NO}_{\mathrm{y}}$ and $\mathrm{NH}_{\mathrm{x}}$ as nitrogen perturbations for the carbon sink calculation. To derive a fossil fuel base for all the models, we used the proportion of $\mathrm{N}$ deposition derived from fossil fuel combustion predicted by IMAGES, which tracks each of the $\mathrm{NO}_{\mathrm{x}}$ sources separately (Lamarque et al. In press), and applied that spatially-distributed proportion to the total $\mathrm{NO}_{\mathrm{y}}$ fields predicted by each 
of the five models. Lightning and stratospheric transport of $\mathrm{N}_{2} \mathrm{O}$, the only two sources which are clearly not influenced by human activity, are between 8 and $20 \%$ of the total NO , sources and between 17 and $39 \%$ of the non-fossil $\mathrm{NO}_{\mathrm{x}}$ sources (Table 3.2).

We next considered total anthropogenic $\mathrm{NO}_{\mathrm{y}}$ by addition of 50 and $90 \%$ of the total non-fossil fuel sources in our calculation of the carbon sink, which provide roughly an additional 10-18 Tg N annually. As discussed above, estimates of important non-fossil fuel emissions of $\mathrm{NO}_{x}$ that contribute substantially to enhanced $\mathrm{NO}_{\mathrm{y}}$ deposition are highly variable. We understand that $90 \%$ may be an overestimate but it reflects possible upward revisions of the global soil $\mathrm{NO}_{\mathrm{x}}$ inventories as new measurements from agricultural sources described above are incorporated. For $\mathrm{NH}_{\mathbf{x}}$, where the proportion of deposition that should be considered a perturbation is even less clear than it is for $\mathrm{NO}_{\mathrm{y}}$, we included $50,60.70$, and $80 \%$ of the total $\mathrm{NH}_{\mathrm{x}}$ deposition to bracket the ratio of pre-industrial to industrial $\mathrm{NH}_{\mathbf{x}}$ emissions of $68 \%$ calculated by Dentener and Crutzen (1994). The calculated carbon storage (described in more detail below) increased linearly as the proportion of $\mathrm{NH}_{\mathrm{x}}$ or $\mathrm{NO}_{\mathrm{y}}$ deposition increased, except when " $N$ saturation" was addressed.

We examined the influence of increasing nitrogen deposition over the last century by deriving a time series for both $\mathrm{NO}_{\mathrm{x}}$ and $\mathrm{NH}_{\mathrm{x}}$ emissions. For both cases, we scaled the $\mathrm{N}$ emissions to a time series of global fossil fuel emissions for the last 130 years (Keeling 1994. Marland et al. 1994). For 1860 to $1950, \mathrm{CO}_{2}$ emissions from each of the four fuel categories. coal, lignite, crude petroleum, and natural gas, were converted to $\mathrm{NO}_{\mathrm{x}}$ emissions by applying the conversion factors given by Müller (1992). The four categories were then summed for each year to provide the total annual estimate. For $1950-1990$, the $\mathrm{NO}_{\mathrm{x}}$ emissions were derived using the Müller (1992) emission factors and the country-by-country fossil fuel statistics for that reporting period. We then calculated a 0-1 scalar for each year to convert the "current" 
emissions provided by each model. The resulting time series, a vector representing an approximately exponential increase in emissions over the last 130 years, was multiplied by the scalar deposition value available for each grid cell. We attempted to scale $\mathrm{NH}_{\mathrm{x}}$ deposition to livestock populations based on a Food and Agriculture Organization (FAO) database, but, because the increase in $\mathrm{NH}_{\mathrm{x}}$ emissions were driven in varying proportions by the livestock contributions, we used the same roughly exponential increase in deposition that we used for $\mathrm{NO}_{\mathbf{y}}$

\subsubsection{Wet deposition validation}

As a first step to determine whether the spatial patterns and quantities in the observations and simulations were comparable, IMAGES simulations of nitrate deposition in precipitation were compared to the average wet nitrate deposition measured at over 200 sites between 1978 and 1994 as part of the US National Atmospheric Deposition Program (NADP/NTN 1995). Neither the spatial distribution nor the magnitude of wet nitrate deposition were adequately captured by IMAGES. First, IMAGES simulated peak deposition over the southeastern US rather than the northeastern region, suggesting problems with transport and rainout parameterization. Second, on a site-to-grid cell comparison, IMAGES consistently underpredicted the amount of nitrate deposited which is best demonstrated by the clear offset in Fig. 3.6 and the $R^{2}$ of 0.55 for the linear correlation. A systematic bias could have been introduced into the comparison by aggregating observations that are skewed toward lower deposition values up to the large grid cells of the simulations, as deposition generally follows a log-normal distribution over large regions (Haas 1990). It is likely that the hot-spots of deposition which cover a limited area can not be represented by such a coarse-grid model. Some inaccuracies in the data (NADP/NTN 1995) resulting from contamination and instrument 


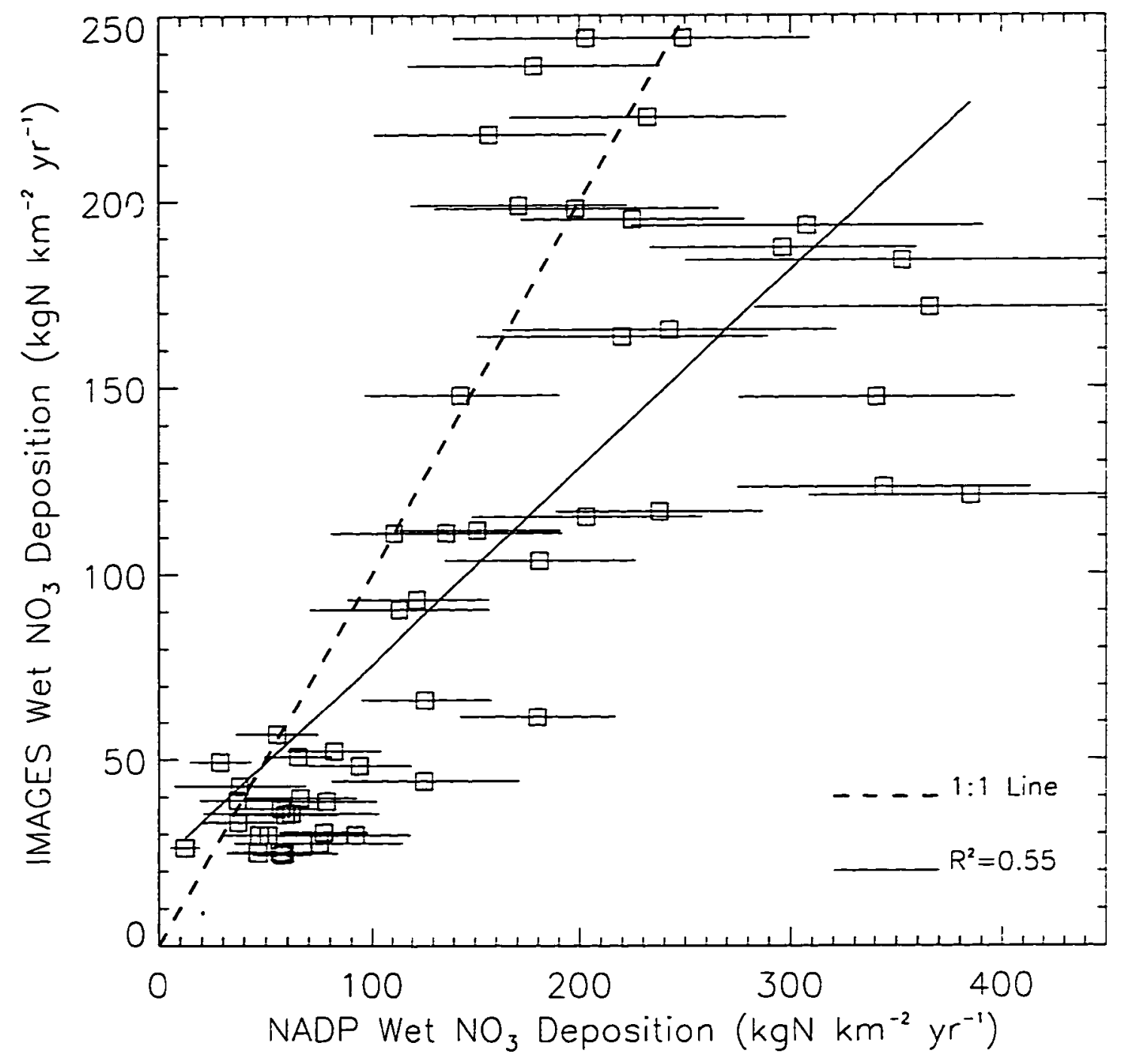

Figure 3.6 A comparison of $\mathrm{NO}_{3}{ }^{-}$deposition in precipitation simulated by IMAGES, and measured at 200 sites within the US by the NADP network (NADP/NTN 1995). All measurements within a $5^{\circ} \times 5^{\circ}$ grid were averaged over the available time period (1978-1994 in most cases). Thus the bars represent the standard deviation including the spatial and temporal variance. Note the different axes scales. 
problems could also contribute to the poor model-data comparison.

The IMAGES-NADP comparison (Fig. 3.6) suggests that transport and rainout portions of the model could be improved, and such improvements are underway (Müller, personal communication). The poor agreement is not a particularly new result, and follows many calls for better parameterization of rainout, particularly of $\mathrm{HNO}_{3}$, and its coupling to transport. These improvements are needed to ensure realistic simulations of atmospheric concentrations of $\mathrm{NO}_{\mathrm{x}}$, as well as $\mathrm{NO}_{\mathrm{y}}$ deposition (Levy and Moxim 1989a,b, Roelofs and Lelieveld 1995, Brasseur et al. In press).

The comparison was a first step towards a more systematic evaluation, and illustrates the problems associated with validation of chemical transport models. Note that the rainout of $\mathrm{HNO}_{3}$ in at least one of the five models (GCTM) was parameterized using NADP data. The observation/model comparison suggests that global $\mathrm{NO}_{\mathrm{y}}$ deposition in precipitation may be higher than simulated. Wet deposition constitutes only $40-60 \%$ of total $\mathrm{N}$ deposition, thereby adding more uncertainty to the model-data relationship (Asman and Jaarsveld 1992, Dentener and Crutzen 1994). The results below should be interpreted knowing that, in at least one case, the details of $\mathrm{N}$ deposition at the spatial scales of ecosystems are simulated poorly.

\subsubsection{Spatial patterns of $\mathrm{NO}_{\mathrm{v}}$ deposition}

All the models simulated different spatial distributions and quantities of $\mathrm{NO}_{\mathrm{y}}$ deposition when all sources of $\mathrm{NO}_{\mathrm{x}}$ were considered (Figs. 3.7, 3.8, and 3.9). Differences amongst the models were significantly reduced when only the fossil fuel sources of $\mathrm{NO}_{\mathrm{y}}$ were considered (Table 3.3). Global $\mathrm{N}$ deposition generated by fossil fuel combustion varied by $10 \%$, in parallel with the variation amongst the magnitude of the assumed fossil fuel sources. The amount of $\mathrm{NO}_{\mathrm{y}}$ deposition derived from fossil fuel combustion differs slightly from the sources because 

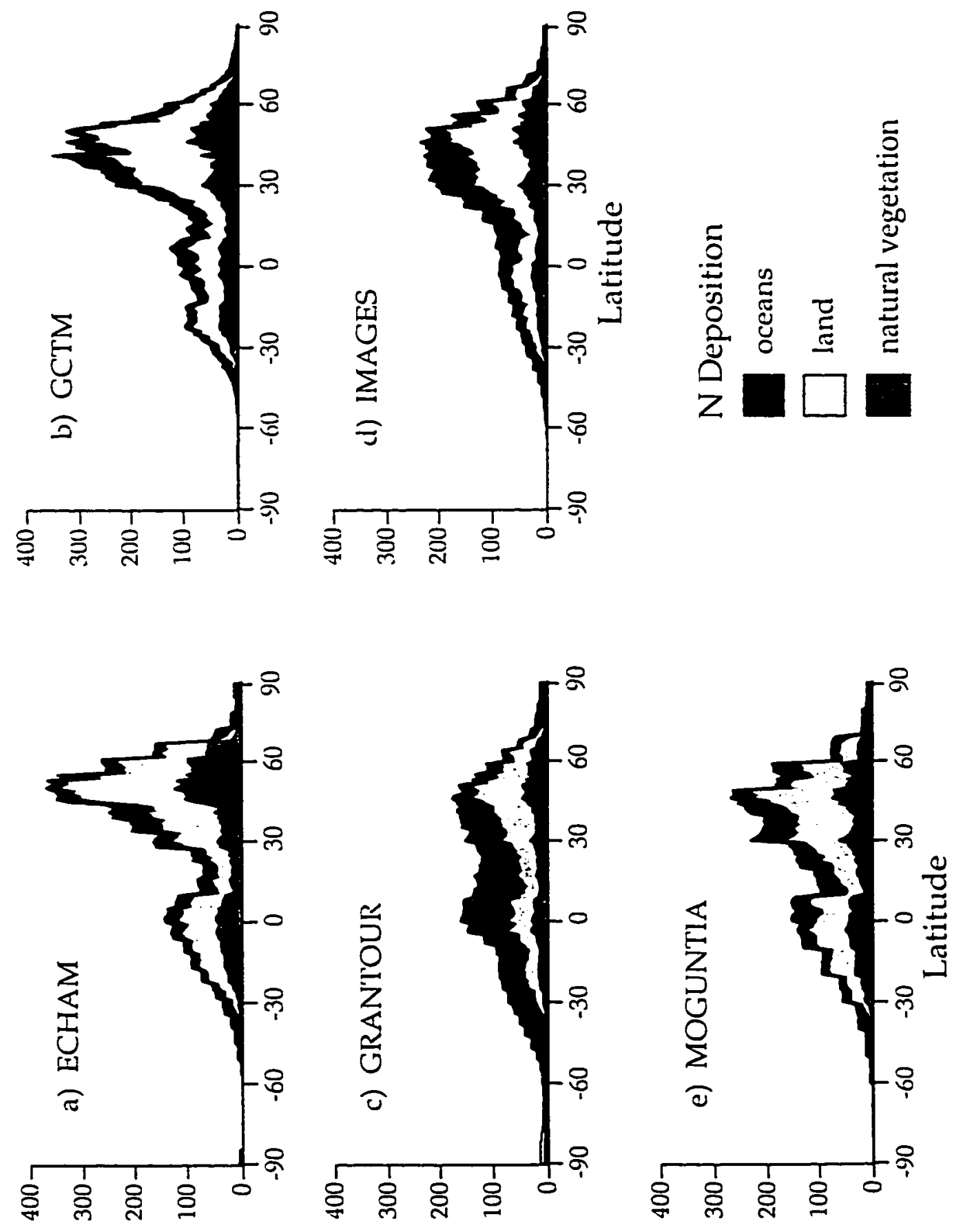

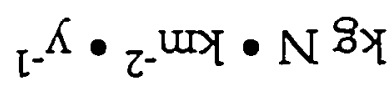

Figure 3.7 The latitudinal distribution of total $\mathrm{NO}_{\mathrm{y}}$ distribution on oceans, land, and natural vegetation for each of the five models used. 
IMAGES NOy deposition

$\mathrm{kg} \mathrm{km}^{-2} \mathrm{yr}^{-1}$

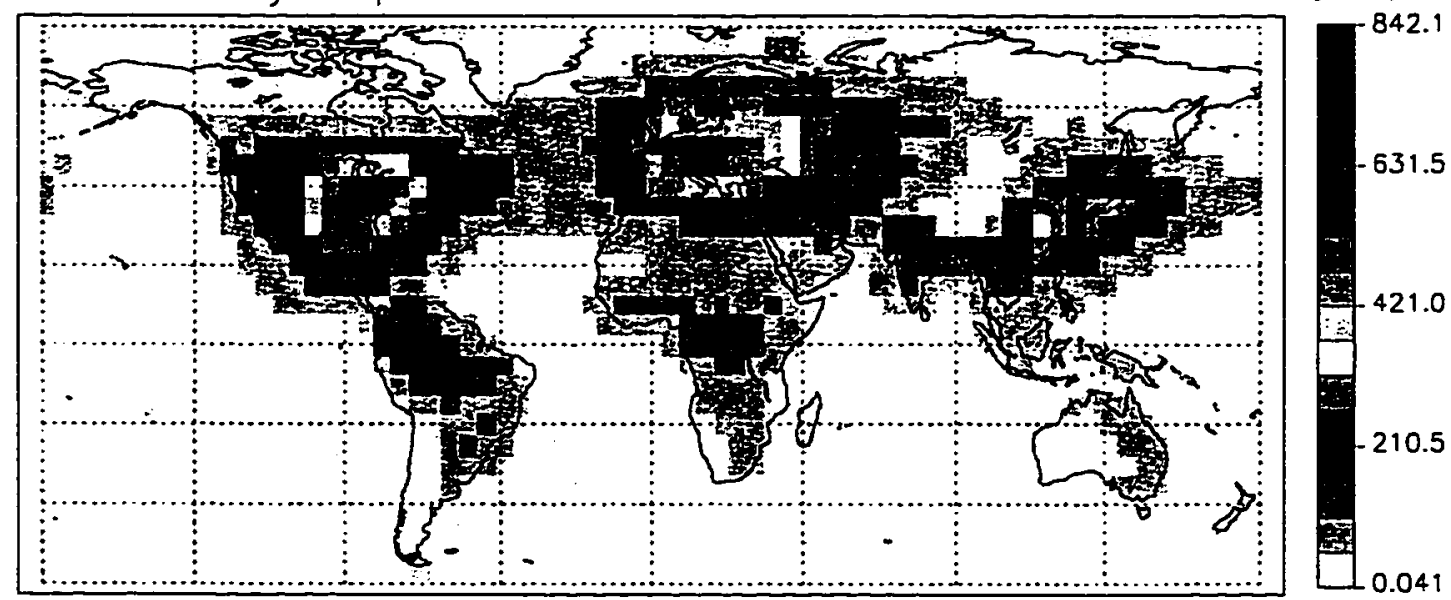

Figure 3.8 Global distribution of total $\mathrm{NO}_{\mathrm{y}}$ deposition simulated by IMAGES in $\mathrm{kg} \mathrm{N} \mathrm{km}^{-2}$ $\mathrm{yr}^{-1}$. 

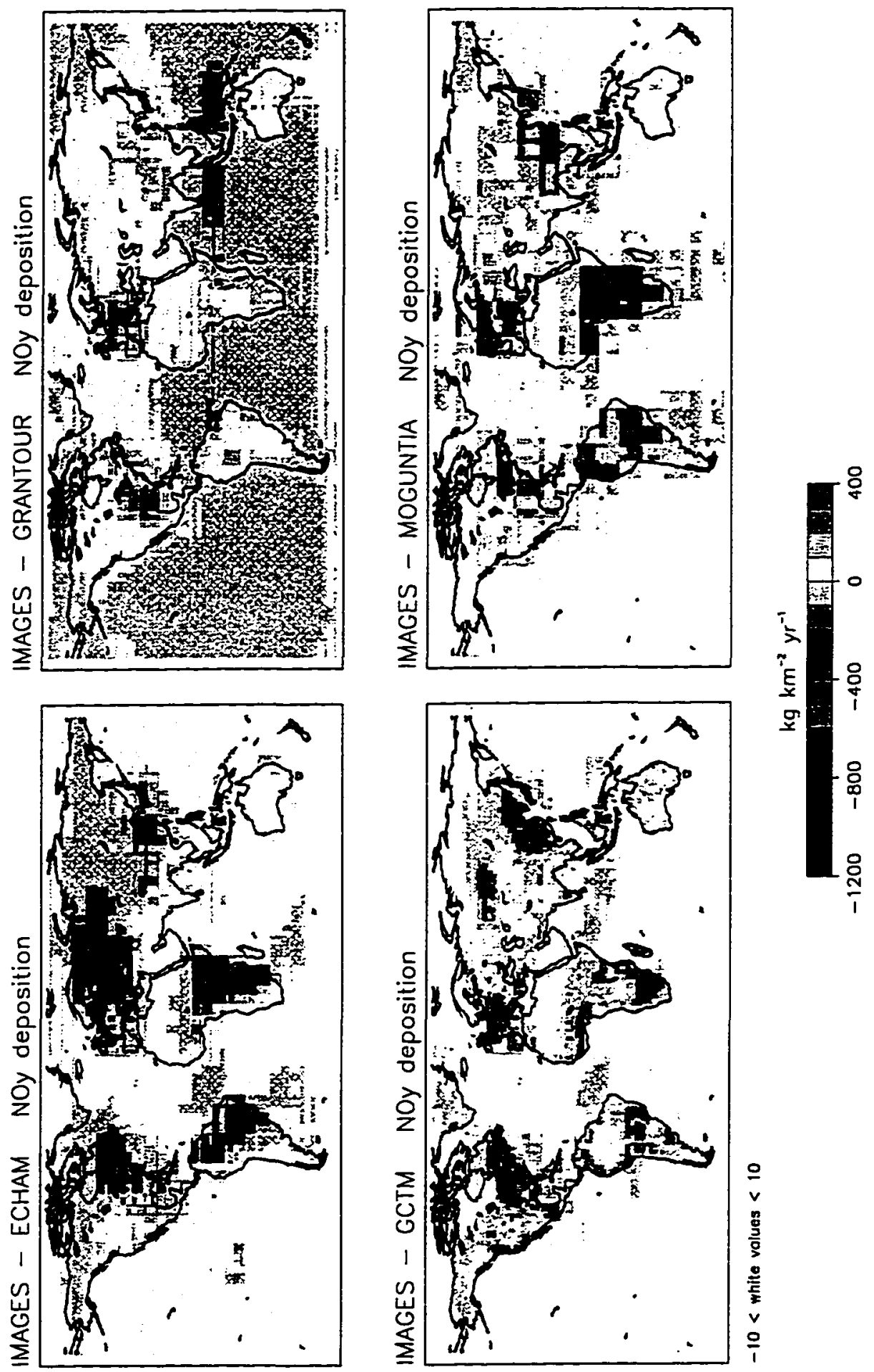

Figure 3.9 Global distributions of the difference between total $\mathrm{NO}_{\mathrm{y}}$ deposition simulated by IMAGES and each of the other models: (a) ECHAM; (b) GCTM; (c) GRANTOUR; (d) MOGUNTIA. All the differences were calculated based on a coregistered $1^{\circ} \times 1^{\circ}$ map of the deposition fields. Warm colors indicate areas where IMAGES deposition was greater than the other model, and cool colors indicate where the other model predicted greater deposition. 
Table 3.3 $\mathrm{NO}_{\mathrm{y}}$ deposition on the Earth's surface resulting from fossil fuel combustion ( $\mathrm{Tg} \mathrm{N}$ $\left.\mathrm{yr}^{-1}\right)$ simulated by five 3-D chemical transport models and the resulting $\mathrm{C}$ sink $\left(\mathrm{Pg} \mathrm{yr}{ }^{-1}\right)$ calculated by NDEP (Townsend et al. 1996).

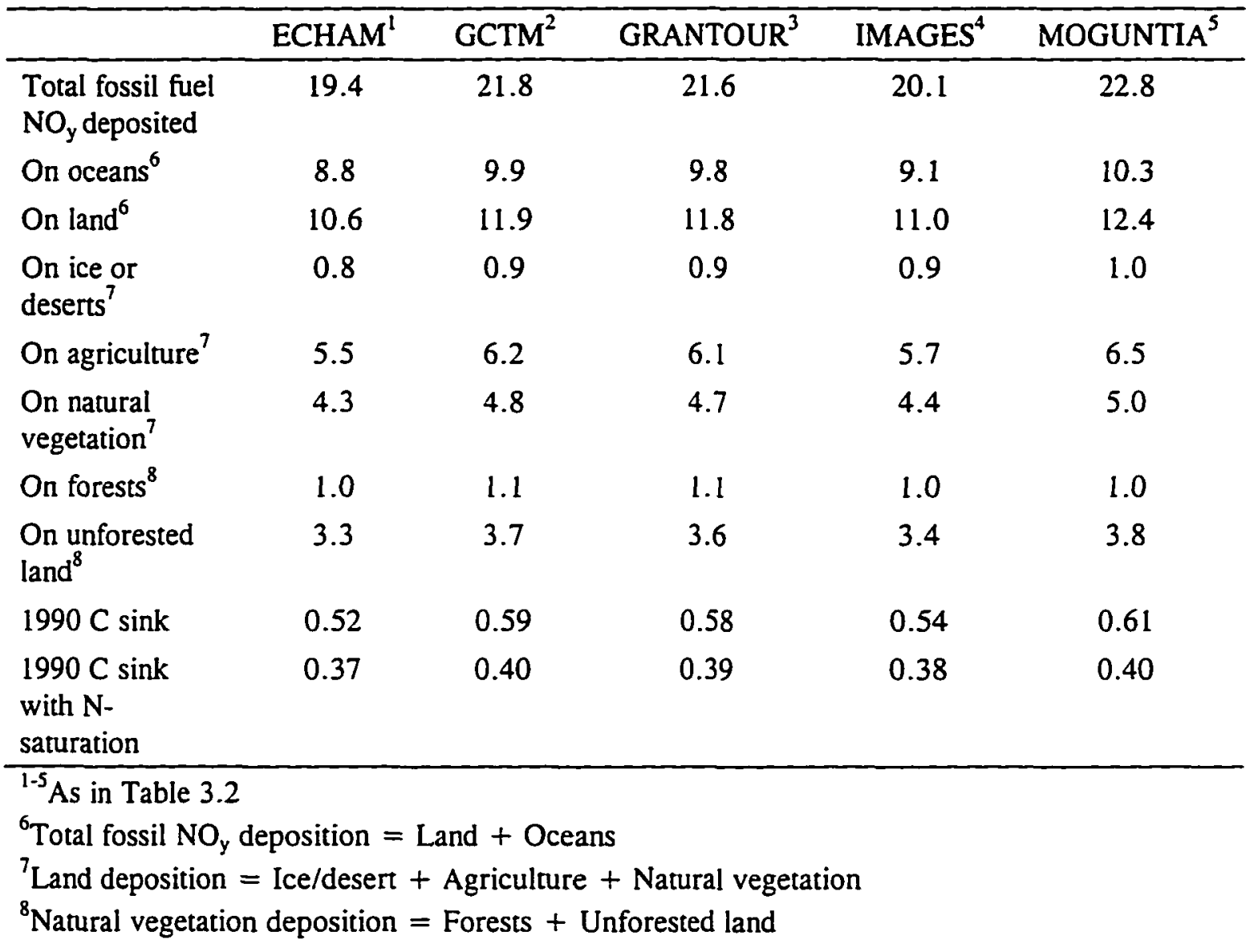

the proportional contributions of each source were calculated using IMAGES and applied to the individual grid cells of all the other models. This technique provided a consistent mechanism with which to deduce the fossil contribution of all the other models. Fossil fuel-derived $\mathrm{NO}_{\mathrm{y}}$ is deposited mainly in the Northern Hemisphere, where it was released, with small amounts transported to the Southern Hemisphere (Galloway et al. 1994, Lamarque et al. In press).

When all the sources of $\mathrm{NO}_{x}$ were considered, the variation in deposition increased considerably: $\mathrm{N}$ deposition ranged between 35.3 (IMAGES) and 41.2 (GRANTOUR) $\mathrm{Tg} \mathrm{yr}^{-1}$ (Fig. 3.7, Table 3.3). In all cases, $\mathrm{NO}_{\mathrm{y}}$ deposition was greatest between $20^{\circ}$ and $60^{\circ} \mathrm{N}$ 
latitude, but a secondary peak of varying height occurred between $10^{\circ} \mathrm{N}$ and $30^{\circ} \mathrm{S}$ latitude (Fig. 3.7). IMAGES and GRANTOUR both deposited less $\mathrm{N}$ on land in the Southern Hemisphere than the other three models. A strong interhemispheric gradient of $\mathrm{NO}_{\mathrm{y}}$ deposition was simulated by all the models, with ECHAM and GCTM simulating the greatest $\mathrm{N}$ deposition in the eastern US, Europe, and Asia (particularly along the northeast coast of China).

Differences amongst the models are greatest in these three regions, followed by portions of Africa and South America (Fig. 3.9). Interestingly, some of the difference in deposition was driven by uncertainties in the biological sources. For all models, the sum of the sources did not equal total global deposition (with differences ranging from -7.6 to $+4.9 \operatorname{Tg~} \mathrm{y} \mathrm{yr}^{-1}$ ) indicating that $\mathrm{N}$ mass was not completely conserved.

For all five models, about $22 \%$ of $\mathrm{NO}_{\mathrm{y}}$ deposition from fossil fuel combustion fell on natural vegetation, and only $5 \%$ of $\mathrm{NO}_{\mathrm{y}}$ deposition fell on forests (Table 3.3). The amount of fossil $\mathrm{NO}_{\mathrm{y}}$ deposited on natural vegetation varied by $17 \%$, slightly more than the variation in fossil fuel sources. Once expanded to include all sources of $\mathrm{NO}_{\mathbf{x}}$, the variability in the spatial deposition increased. Absolute quantities of $\mathrm{N}$ deposition on forests ranged from 2.2 to $4.0 \mathrm{Tg}$ $\mathrm{yr}^{-1}$ (Table 3.4). The proportion of total global $\mathrm{N}$ deposition received by forests, which store large amounts of carbon in wood varied between 5.3\% (GRANTOUR) and 10\% (ECHAM), with IMAGES (6.6\%), MOGUNTIA (8.9\%), and GCTM $(9 \%)$ in between. The ratio of forest to non-forest deposition ranged between 0.37 (IMAGES) and 0.42 (GCTM and MOGUNTIA) with GRANTOUR (0.41) and ECHAM $(0.40)$ in between. This variation in the spatial distribution of $\mathrm{N}$ deposition was a primary factor in determining the size and range of the resulting carbon sink when sources other than fossil fuel were considered (Fig. 3.10). 
Table 3.4 Total $\mathrm{NO}_{\mathrm{y}}$ deposition on the Earth's surface considering all $\mathrm{NO}_{\mathrm{x}}$ sources $\left(\mathrm{Tg} \mathrm{N} \mathrm{yr}^{-\mathrm{t}}\right)$ simulated by five 3-D chemical transport models.

\begin{tabular}{|c|c|c|c|c|c|}
\hline & $\mathrm{ECHAM}^{1}$ & $\mathrm{GCTM}^{2}$ & GRANTOUR $^{3}$ & IMAGES $^{4}$ & MOGUNTIA $^{5}$ \\
\hline $\begin{array}{l}\text { Global } \mathrm{NO}_{\mathrm{y}} \\
\text { deposition }\end{array}$ & 38.9 & 40.2 & 41.2 & 35.3 & 39.9 \\
\hline On oceans $^{6}$ & 11.7 & 12.6 & 25.8 & 16.2 & 14.9 \\
\hline On land ${ }^{6}$ & 27.2 & 27.6 & 15.4 & 19.1 & 25.0 \\
\hline $\begin{array}{l}\text { On ice or } \\
\text { deserts }^{7}\end{array}$ & 1.2 & 1.2 & 1.2 & 1.4 & 1.4 \\
\hline On agriculture ${ }^{7}$ & 12.2 & 14.1 & 6.6 & 8.9 & 11.4 \\
\hline $\begin{array}{l}\text { On natural } \\
\text { vegetation }\end{array}$ & 13.8 & 12.3 & 7.7 & 8.8 & 12.1 \\
\hline On forests ${ }^{8}$ & 4.0 & 3.6 & 2.2 & 2.3 & 3.6 \\
\hline $\begin{array}{l}\text { On unforested } \\
\text { land }^{8}\end{array}$ & 9.8 & 8.6 & 5.4 & 6.5 & 8.5 \\
\hline $\begin{array}{l}\text { Deposition } \\
\text { minus } \\
\text { emissions }^{9}\end{array}$ & -1.1 & +1.6 & -7.6 & -7.3 & +4.9 \\
\hline $\begin{array}{l}1-5 \text { As in Table } 3.2 \\
{ }^{6-8} \text { As in Table } 3.3\end{array}$ & & & & & \\
\hline
\end{tabular}

\subsubsection{Carbon storage from NOy deposition}

Considering only fossil fuel-derived $\mathrm{NO}_{\mathrm{y}}$ deposition, the globally integrated annual carbon sink for all the models ranged from 0.43-0.61 $\mathrm{Pg} \mathrm{yr}^{-1}$ (Table 3.3). These values represent approximately $25-33 \%$ of the "missing sink" (Schimel et al. 1995. Schimel 1995b). When additional sources of $\mathrm{NO}_{\mathbf{x}}$ were considered, the variation in the size of the estimated carbon sink grew more than threefold. and partially depended on the proportion of non-fossil sources of $\mathrm{NO}_{\mathrm{x}}$ considered (Table 3.4; Fig. 3.10a). When fossil fuel sources of $\mathrm{NO}_{\mathrm{x}}$ plus $50 \%$ of non-fossil sources were considered, the calculated carbon sink ranged from $0.65 \mathrm{Pg} \mathrm{yr}^{-1}$ for GRANTOUR to $0.97 \mathrm{Pg} \mathrm{yr}^{-1}$ for ECHAM (Fig. 3.10a). When $90 \%$ of non-fossil sources were considered, the calculated carbon sink ranged from $0.72 \mathrm{Pg} \mathrm{yr}^{-1}$ for GRANTOUR to $1.34 \mathrm{Pg}$ 

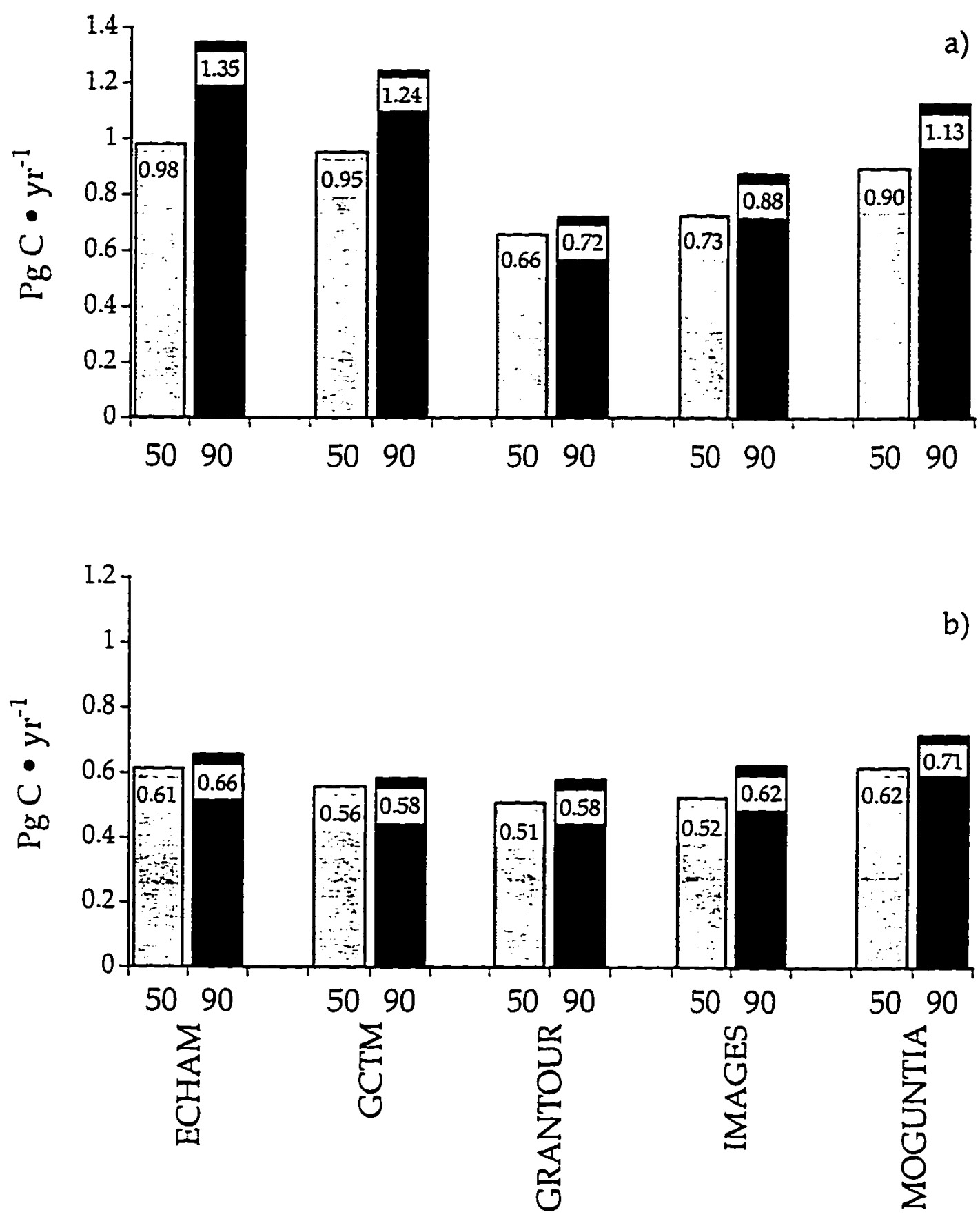

Figure 3.10 Globally integrated carbon storage calculated by NDEP from $\mathrm{NO}_{y}$ deposition considering $\mathrm{NO}_{\mathrm{x}}$ emissions from fossil fuel combustion plus 50 and $90 \%$ of non-fossil sources for each of the five models. (a) $N_{\text {loss }}=0.2 \cdot N_{a v}$. i.e., $80 \%$ of available $\mathrm{N}$ is assimilated. The remaining $20 \%$ is assumed to be transferred back to the atmosphere $\mathrm{NO}, \mathrm{N}_{2}, \mathrm{~N}_{2} \mathrm{O}$, or in hydrologic systems. (b) $N_{\text {loss }}=a+b \cdot N_{a v}$, where $a=0.2$ and $b=0.8 / 1000$. Modeled ecosystems utilized an increasingly smaller proportion of the deposited $\mathrm{N}$ (the " $\mathrm{N}$ saturation" case). 
$\mathrm{yr}^{-1}$ for ECHAM. The size of the $\mathrm{C}$ sink was proportional to the amount of $\mathrm{N}$ deposited on natural vegetation and was closely linked to the relatively small amount of $\mathrm{N}$ deposited on forests $(<10 \%)$ because carbon storage in wood dominates all other storage compartments (Tables 3.3 and 3.4; Townsend et al. 1996).

To consider how nitrogen saturation might influence enhanced terrestrial carbon uptake, we followed the same procedure ourlined in Townsend et al. (1996). We conducted a set of NDEP simulations that included a simple parameterization of a reduction in ecosystem nitrogen use efficiency in response to chronic deposition. The fraction of $\mathrm{N}$ retained was decreased linearly as a function of deposition down to a critical threshold where inputs equal outputs and none of the additional deposited $\mathrm{N}$ was retained by the ecosystem (see Section 3.4). The result was to effectively place a cap on the $\mathrm{N}$-induced carbon sink to between 0.5 and 0.6 Pg yr ${ }^{-1}$ (Fig. 3.10b).

\subsubsection{Effects of NHx deposition}

Emissions of ammonia represent at least as large a flux of $\mathrm{N}$ to the atmosphere as $\mathrm{NO}_{\mathrm{x}}$ (Dentener and Crutzen 1994). As pointed out in Table 3.2, global NO emissions to the atmosphere are estimated to be 35 to $49 \mathrm{Tg} \mathrm{N} \mathrm{yr}^{-1}$ for the five models, and $\mathrm{NH}_{\mathrm{x}}$ emissions are estimated to be $45 \mathrm{Tg} \mathrm{N} \mathrm{yr}^{-1}$ (Dentener and Crutzen 1994). The only three-dimensional chemical model to date that considers ammonia is an updated version of MOGUNTIA developed by Dentener and Crutzen (1994); we used this version to estimate the size of the carbon sink due to $\mathrm{NH}_{\mathrm{x}}$. Total $\mathrm{NH}_{\mathrm{x}}$ deposition in this model was $41 \mathrm{Tg} \mathrm{N} \mathrm{yr} r^{-1}$. As with $\mathrm{NO}_{\mathrm{y}}$ deposition, $\mathrm{NH}_{\mathrm{x}}$ deposition was greater on land $\left(22.5 \mathrm{Tg} \mathrm{N}_{\mathrm{yr}} \mathrm{r}^{-1}\right)$ than on oceans (18 $\mathrm{Tg} \mathrm{N} \mathrm{yr}^{-1}$ ) (Fig. 3.11a).

The partitioning of $\mathrm{NH}_{\mathrm{x}}$ deposition between the various land categories was (in $\mathrm{Tg} \mathrm{N}$ 


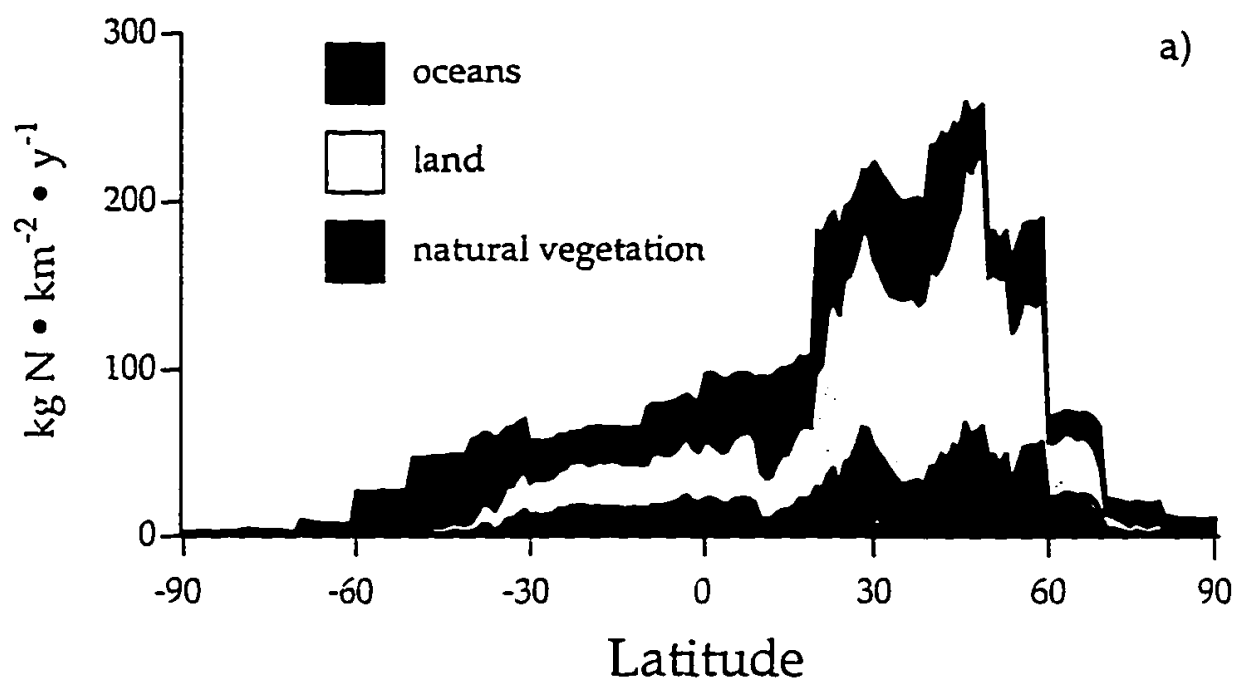

b)

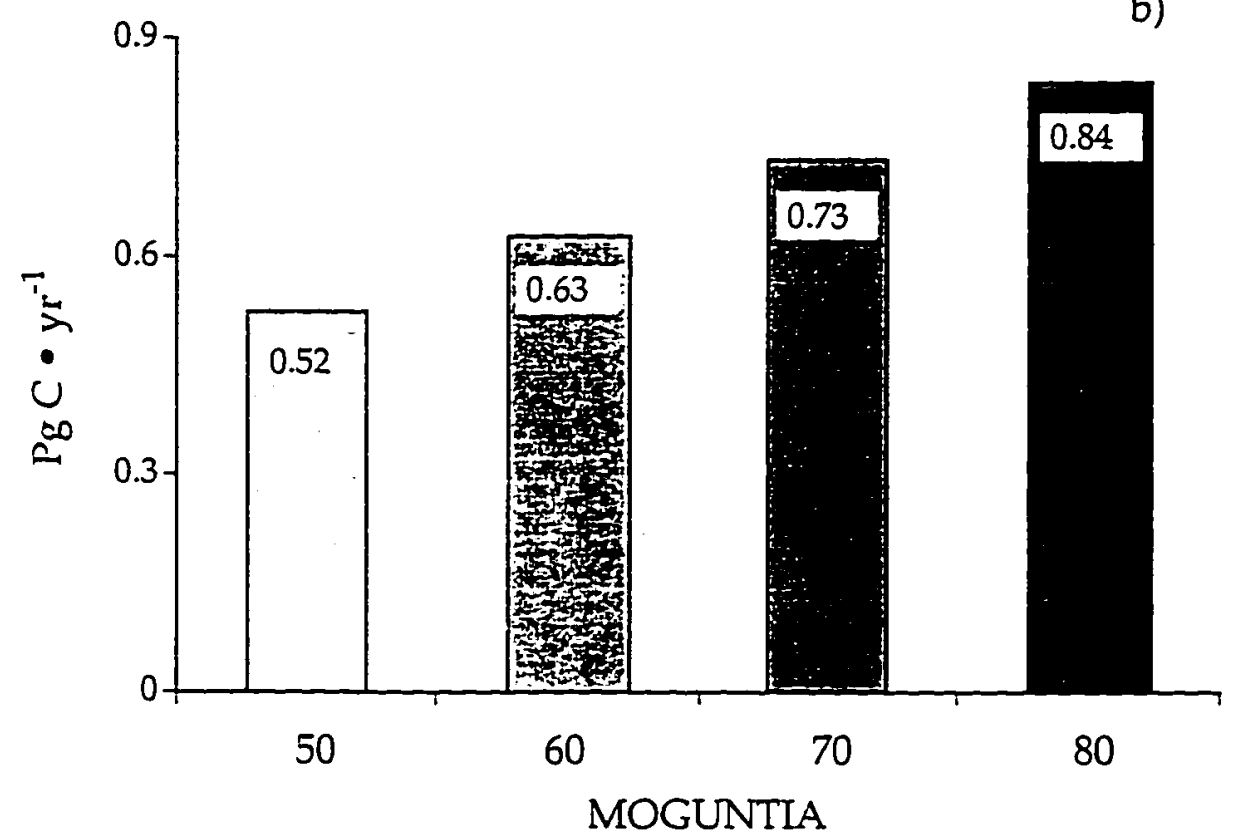

Figure 3.11 (a) Latitudinal distribution of total $\mathrm{NH}_{\mathrm{x}}$ deposition on the Earth's surface, including oceans, land, and natural vegetation. (b) Globally-integrated carbon storage calculated by NDEP considering $50-80 \%$ of the $\mathrm{NH}_{\mathrm{x}}$ deposition simulated by MOGUNTIA (Dentener and Crutzen 1994). The $\mathrm{C}$ sink calculations assumed no $\mathrm{N}$ saturation (80\% ecosystem nitrogen retention). 
$\left.\mathrm{yr}^{-1}\right): 1.2$ on ice or deserts, 11.0 on agriculture, and 10.1 on natural vegetation, which could further be partitioned into 7.3 on forested land and 2.9 on forests. Thus, only $7 \%$ of the total $\mathrm{NH}_{\mathrm{x}}$ deposited fell on forests. The partitioning of $\mathrm{NH}_{\mathrm{x}}$ deposition was likely to have been influenced by specific attributes of MOGUNTIA and its parameterization. First, MOGUNTIA applies a dry deposition factor of $25 \%$ to anthropogenic emissions to account for sub-grid deposition within the rather large $10^{\circ} \times 10^{\circ}$ grid cells. While this may result in overestimation of $\mathrm{NH}_{\mathrm{x}}$ deposition in some non-agricuitural areas, the uncertainties associated with the actual $\mathrm{NH}_{\mathrm{x}}$ emission estimates are likely to override any bias in deposition introduced by the anthropogenic deposition parameterization. For example, Schlesinger and Hartley (1992) estimate global $\mathrm{NH}_{3}$ emissions to $75 \mathrm{Tg} \mathrm{N} \mathrm{yr}^{-1}$, substantially greater than the $45 \mathrm{Tg} \mathrm{N} \mathrm{yr}^{-1}$ used here. Galloway et al. (1995) estimated $\mathrm{NH}_{\mathrm{x}}$ emissions to be $68 \mathrm{Tg} \mathrm{N} \mathrm{yr}^{-1}$. Also, in some areas of the world and particularly in the tropics, domestic animals eat an unknown proportion of natural vegetation (their emissions would not be considered an anthropogenic perturbation). Despite these concerns, MOGUNTIA has examined the consistency of estimated emissions with measured deposition to produce an internally consistent look at the global $\mathrm{NH}_{\mathrm{x}}$ cycle.

The size of the carbon sink induced by the deposition of $\mathrm{NH}_{\mathrm{x}}$ ranged from 0.52 to 0.84 $\mathrm{Pg} \mathrm{yr}^{-1}$, depending on the proportion of the deposition assumed to be anthropogenic (50\% and $80 \%$, respectively) (Fig. 3.11b). Ammonia emissions have risen exponentially over the last century (Nevison et al. 1996) and clearly some fraction of the emissions, which are largely derived from agricultural and animal husbandry practices, represent an increase over preindustrial times when the Earth supported a much smaller population of humans. However, the exact proportion attributable to human activity is extremely difficult to assess. Dentener and Crutzen (1994) estimated anthropogenic emissions to be 30.6 out of the $45 \mathrm{Tg} \mathrm{N} \mathrm{yr}^{-1}$, or $68 \%$, and Galloway et al. (1995) arrived at an estimate of $69 \%$. 

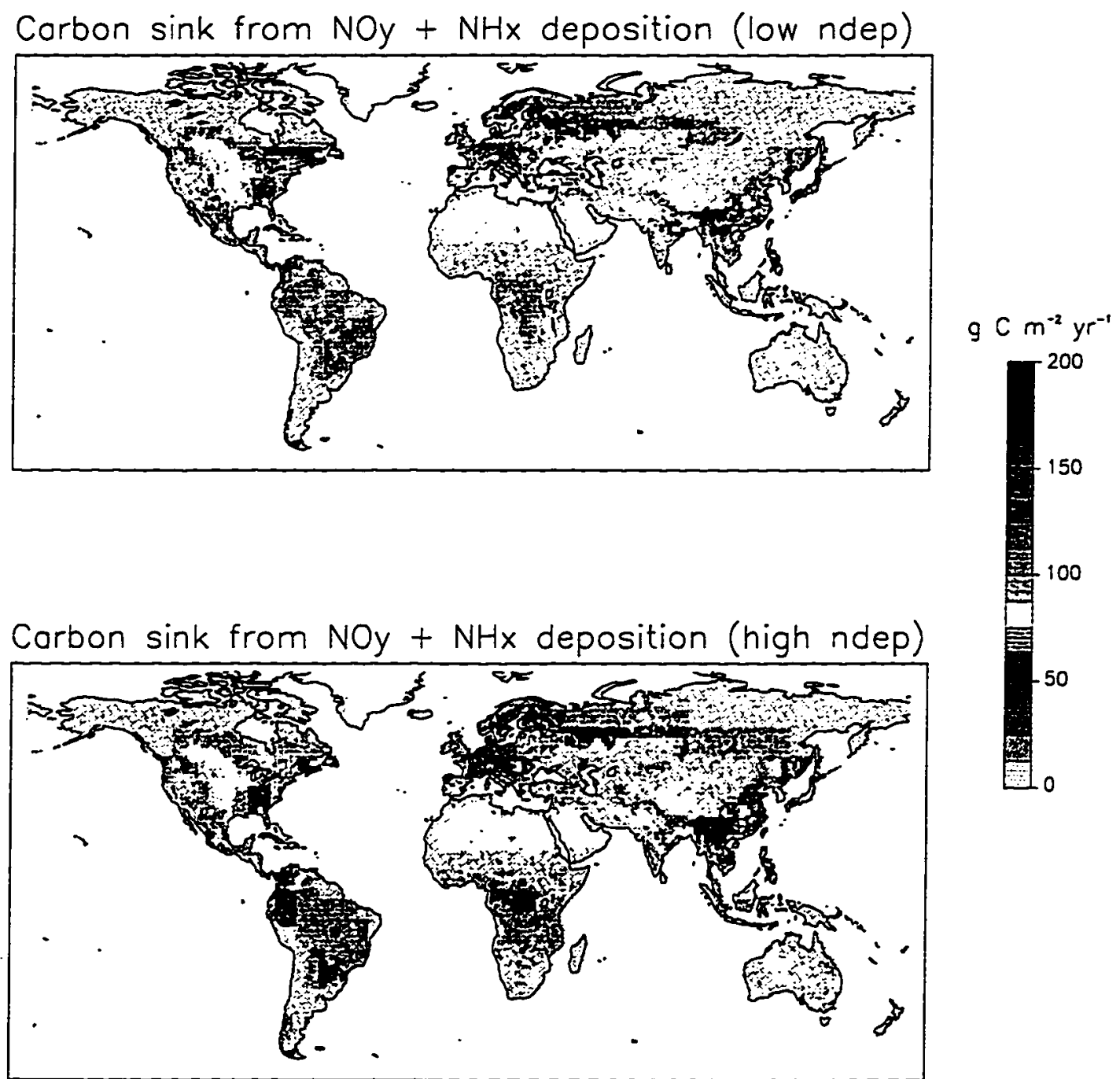

Figure 3.12 Map of the global distribution of carbon storage calculated by NDEP considering both the $\mathrm{NO}_{\mathrm{y}}$ and $\mathrm{NH}_{\mathrm{x}}$ simulated by MOGUNTIA. (a) considering fossil fuel $\mathrm{NO}_{\mathrm{y}}$ plus $50 \%$ non-fossil $\mathrm{NO}_{\mathrm{y}}$ plus $50 \%$ of $\mathrm{N}$ from $\mathrm{NH}_{3}$ emissions. (b) Considers fossil fuel $\mathrm{NO}_{\mathrm{y}}$ plus $90 \%$ non-fossil $\mathrm{NO}_{y}$ plus $95 \%$ of $\mathrm{N}$ from $\mathrm{NH}_{3}$ emissions. For both simulations, we assumed no $\mathrm{N}$ saturation. Globally integrated $C$ storage ranged from $1.2-1.6 \mathrm{Pg} \mathrm{yr}^{-1}$. 
The spatial distribution of $\mathrm{NH}_{\mathrm{x}}$ deposition and the resulting sink are different than those generated for $\mathrm{NO}_{\mathrm{y}}$ deposition (Fig. 3.12). The most striking difference was the enhanced $\mathrm{N}$ deposition (and $C$ sink) in the tropics, particularly India, China, Central America, and northern South America. The consideration of $\mathrm{NH}_{\mathrm{x}}$ emissions also resulted in increased $\mathrm{N}$ deposition between $30^{\circ}$ and $45^{\circ} \mathrm{N}$ latitude compared with $\mathrm{NO}_{\mathrm{y}}$ deposition alone. As a result, $\mathrm{N}$ deposition intensified for the low latitudes of the Northern Hemisphere for $\mathrm{NH}_{\mathrm{x}}$ relative to $\mathrm{NO}_{\mathrm{y}}$ deposition which, not surprisingly, mimics the animal distributions. North of $40^{\circ} \mathrm{N}$ the pattern of $\mathrm{NH}_{x}$ deposition was very similar to that of $\mathrm{NO}_{\mathrm{y}}$ deposition due to a mix of agriculture and industry in the northern temperate zone (Fig. 3.11a) (Chameides et al. 1994). Estimates of the annual global flux of $\mathrm{NH}_{\mathrm{x}}$ are uncertain and the larger estimates could generate a still larger carbon sink depending on its spatial distribution.

When both $\mathrm{NO}_{\mathrm{y}}$ and $\mathrm{NH}_{\mathrm{x}}$ were included in the NDEP simulations, the range for our $\mathrm{C}$ uptake estimates grew to $1.42-1.97 \mathrm{Pg} \mathrm{yr}^{-1}$. The $\mathrm{NO}_{\mathrm{y}}$ perturbation included fossil fuel sources of $\mathrm{NO}_{\mathrm{x}}$ and 50 and $90 \%$ of the non-fossil sources, respectively, and the $\mathrm{NH}_{\mathrm{x}}$ perturbation included 50 and $80 \%$ of the total $\mathrm{NH}_{\mathrm{x}}$ sources, respectively. In addition, we assumed that $\mathrm{C}$ fixation was not hampered due to $\mathrm{N}$ saturation in regions of high chronic deposition. The inclusion of negative feedbacks on $\mathrm{N}$ retention associated with $\mathrm{N}$ saturation severely constrained modeled $\mathrm{CO}_{2}$ uptake, reducing the sink to $1.08-1.35 \mathrm{Pg} \mathrm{C} \mathrm{yr}^{-1}$.

\subsubsection{Relation of this study to other analyses}

This study is only the second (after Townsend et al. 1996) to explicitly consider the spatial distribution of $\mathrm{N}$ deposition on ecosystems. In 1985, Peterson and Melillo presented an estimate of carbon uptake by forest vegetation and soils due to $\mathrm{N}$ fertilization of $0.1 \mathrm{Pg} \mathrm{yr}^{-1}, \mathrm{a}$ relatively small sink and less than one-fifth our current estimate for either $\mathrm{NO}_{\mathrm{y}}$ or $\mathrm{NH}_{\mathrm{x}}$ 
deposition and less than one-tenth our total carbon sink estimate. They assumed that a total of 6 $\mathrm{Tg}$ out of $20 \mathrm{Tg} \mathrm{N}$ released by fossil fuel combustion was deposited on natural vegetation, an amount slightly higher than our estimate of 4.3-5.0 $\mathrm{Tg} \mathrm{N}$ (Table 3.3). The present study is distinguished by: (1) simulation of the $\mathrm{N}$ cycling of specific land cover types; (2) derivation of the spatial distribution of $\mathrm{N}$ deposition using chemical transport models; (3) evaluation of the cumulative effect of rising $\mathrm{N}$ deposition and recycling of deposited $\mathrm{N} ;(4)$ an assumption that $80 \%$ of the deposited $\mathrm{N}$ is retained, compared with $60 \%$ (Peterson and Melillo 1985); and (5) the incorporation of $\mathrm{C}: \mathrm{N}$ ratios for different pools, which reflect the intervening decade of measurements and subsequent understanding.

More recent estimates of the carbon sink caused by anthropogenic $\mathrm{N}$ deposition range from 0.50 to $1.50 \mathrm{Pg} \mathrm{yr}^{-1}$ (Kohlmaier et al. 1988, Hudson et al. 1994, Schindler and Bayley 1993, Townsend et al. 1996). Hudson et al. (1994) simulated a carbon sink of $1.4 \mathrm{Pg} \mathrm{yr}^{-1}$ with $\mathrm{N}$ deposition on temperate and boreal forests of $18 \mathrm{Tg} \mathrm{yr}^{-1}$. The five chemical transport models studied here simulate between 2.2 and $4.0 \mathrm{Tg} \mathrm{N} \mathrm{yr}^{-1}$ as $\mathrm{NO}_{\mathrm{y}}$ and $2 \mathrm{Tg} \mathrm{N} \mathrm{yr} r^{-1}$ as $\mathrm{NH}_{\mathrm{x}}$ on all forests, including temperate, boreal, and tropical forests (fossil fuel plus all other sources; Table 3.4). Schindler and Bayley (1993) estimated a carbon sink of $0.65-1.95 \mathrm{Pg} \mathrm{yr}^{-1}$ on land, depending on the assigned $\mathrm{C}: \mathrm{N}$ of the terrestrial biosphere, and assuming global $\mathrm{N}$ deposition on land of $13 \mathrm{Tg} \mathrm{yr}^{-1}$, which is similar to our fossil fuel $\mathrm{NO}_{\mathrm{y}}$ estimate of $10.6-12.4 \mathrm{Tg} \mathrm{N} \mathrm{yr}^{-1}$ deposited on land globally. The estimates of Schindler and Bayley (1993) and Hudson et al. (1994) differ from ours in the assignment of C:N ratios, assumptions about the geography of $\mathrm{N}$ deposition and ecosystems, and (lack of) consideration of $\mathrm{N}$ recycling.

The analysis of atmospheric $\mathrm{CO}_{2}$ and $\delta^{13} \mathrm{C}$ has provided another perspective on the global carbon cycle and has allowed a more robust determination of the latitudinal distribution of net terrestrial $\mathrm{CO}_{2}$ exchange (e.g. . Ciais et al. 1995a; see also Section 2.2). The latitudinal 
distribution of the carbon sink derived from $\mathbf{N}$ deposition is similar to the latitudinal distribution derived from isotopic/concentration measurements coupled to an inverse modeling framework (Fig. 3.13; Ciais et al. 1995a). The inverse model estimates suggest a Northern Hemisphere mid-latitude terrestrial sink, which all of our estimates also show (Table 3.5). When we include the non-fossil $\mathrm{NO}_{\mathrm{x}}$ and $\mathrm{NH}_{\mathrm{x}}$ sources, southern subtropical carbon uptake is similar to that estimated by Ciais et al. (1995a). The intensity of both the Northern Hemisphere and subtropical carbon sinks varies amongst the models with GCTM. ECHAM, and MOGUNTIA showing the strongest Northern Hemisphere sink and ECHAM and MOGUNTIA showing the strongest subtropical sink. The nitrogen deposition-induced sink is not inconsistent with the data-based inverse estimates, suggesting that the role of $\mathrm{N}$ deposition in the carbon cycle should be investigated further, using empirical as well as more mechanistic modeling approaches.

Our estimates of the $\mathrm{N}$-induced terrestrial carbon sink reflect our current understanding of both atmospheric chemistry and ecological function. Likewise, the causes of uncertainty in these estimates indicate specific areas that require further and more detailed investigation. Outstanding examples of factors that can substantially influence the size and distribution of the C sink include the mapping of vegetation types, the amount of carbon assimilation allocated to woody biomass, and the issue of nitrogen saturation and potential chemical feedbacks (Section 3.4). All of these factors are likely to be spatially and temporally variable. Sensitivity analysis of these factors generate large ranges in the size of the $\mathrm{C}$ sink estimate, but it is almost certain that the $\mathrm{N}$-fertilization phenomenon is an important one in the carbon budget. Whether it will remain significant in the face of chronic deposition is unclear. Improved estimates and predictions will be available only through increased understanding of both atmospheric chemistry and ecosystem biogeochemistry. 

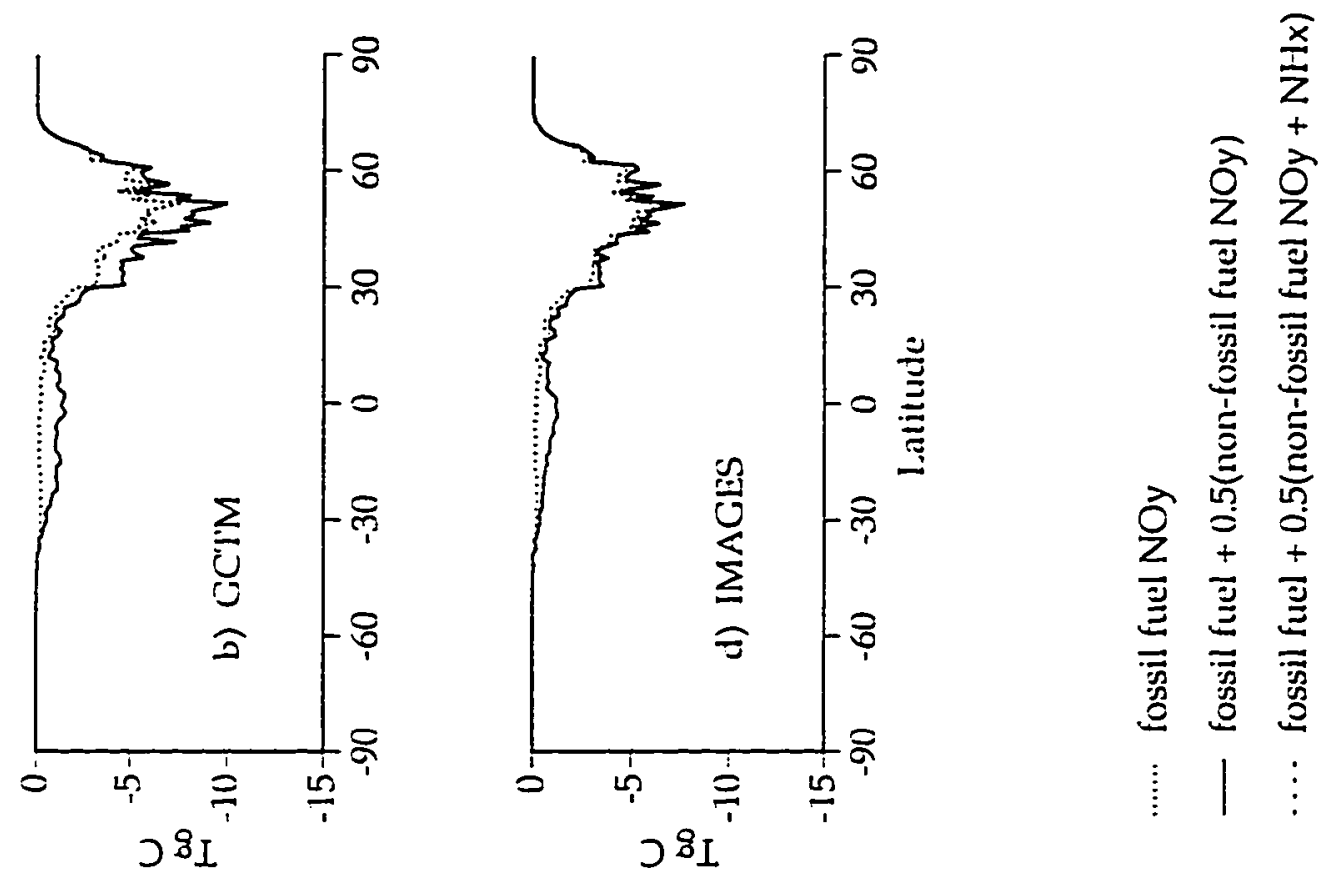

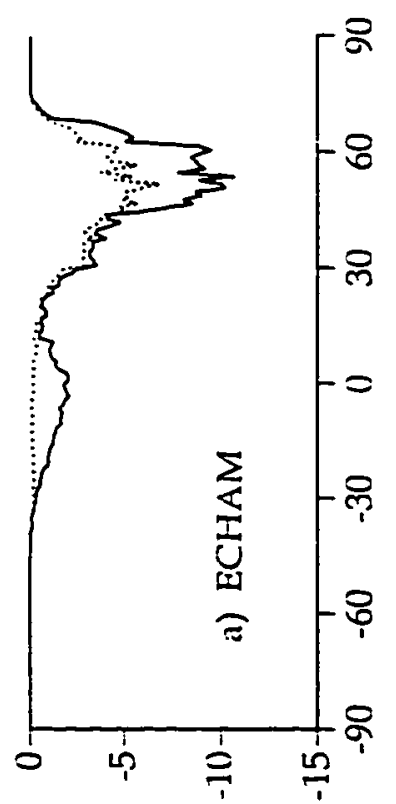

ว 81

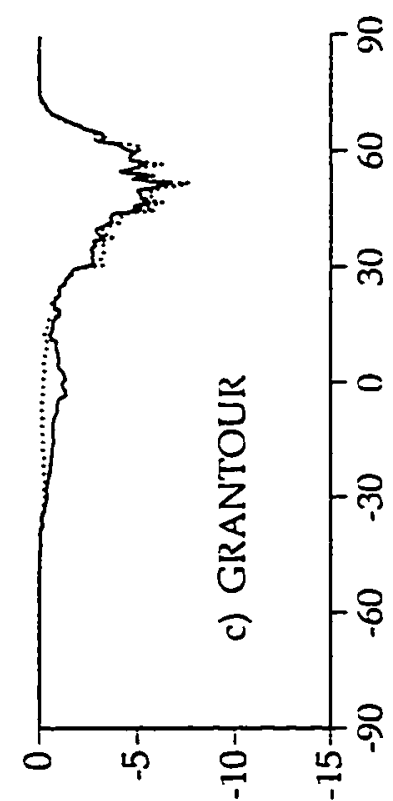

$3^{81}$

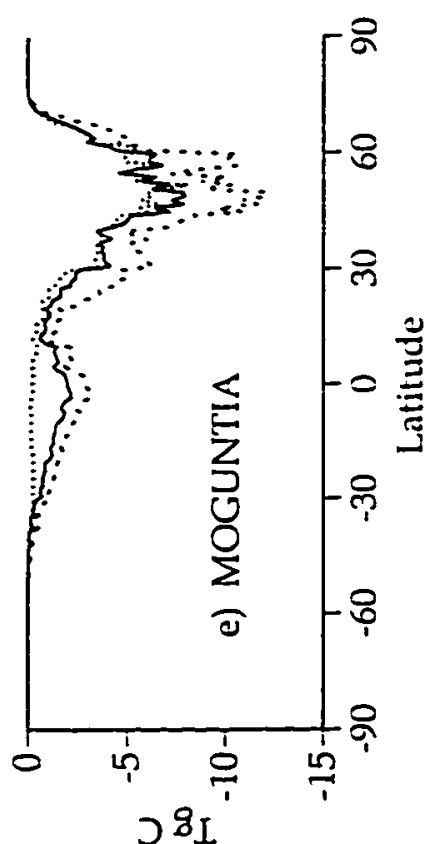

ว

Figure 3.13 Latitudinal distribution of the carbon sink considering $\mathrm{NO}_{\mathrm{y}}$ deposition derived from fossil fuel combustion and considering $\mathrm{NO}_{\mathrm{y}}$ deposition derived from fossil fuel combustion plus $50 \%$ of non-fossil sources for each of the five models. For MOGUNTIA we consider deposition derived from fossil fuel combustion plus $50 \%$ of non-fossil sources plus $50 \%$ of $\mathrm{NH}_{\mathrm{x}}$. A constant $\mathrm{N}$ retention of $80 \%$ was assumed. 
Table 3.5 Comparison of terrestrial net $\mathrm{CO}_{2}$ flux $\left(\mathrm{Pg} \mathrm{C} \mathrm{yr}^{-1}\right)$ estimated by inverse modeling and our perturbation estimate of terrestrial net $\mathrm{CO}_{2}\left(\mathrm{Pg} \mathrm{C} \mathrm{yr}^{-1}\right)$ uptake due to anthropogenic $\mathrm{N}$ deposition. We include $\mathrm{NO}_{y}$ deposition from fossil fuel combustion plus $50 \%$ of non-fossil sources, except for MOGUNTIA $\mathrm{NO}_{\mathrm{y}}+\mathrm{NH}_{\mathrm{x}}$ where we include $50 \%$ of $\mathrm{NH}_{\mathrm{x}}$ deposition.

\begin{tabular}{lcccc}
\hline & $90^{\circ} \mathrm{S}-16^{\circ} \mathrm{S}$ & Equatorial & $16^{\circ} \mathrm{N}-90^{\circ} \mathrm{N}$ & Global \\
\hline Keeling et al. $1989 \mathrm{~b}^{1}$ & -0.1 & +0.3 & -0.6 & -0.5 \\
Tans et al. $1994^{1}$ & -0.1 & +0.5 & -2.3 & -1.9 \\
Ciais et al. $1995 \mathrm{a}^{2}$ & -0.2 & +0.8 & -2.2 & -1.5
\end{tabular}

This work: ${ }^{3}$

With $N$-saturation

\begin{tabular}{|c|c|c|c|c|}
\hline IMAGES & 0.04 & 0.11 & 0.38 & 0.53 \\
\hline ECHAM & 0.05 & 0.16 & 0.41 & 0.61 \\
\hline GCTM & 0.06 & 0.13 & 0.37 & 0.56 \\
\hline GRANTOUR & 0.04 & 0.11 & 0.36 & 0.51 \\
\hline MOGUNTIA & 0.05 & 0.16 & 0.40 & 0.61 \\
\hline MOGUNTIA NH $_{x}+\mathrm{NO}_{y}$ & 0.10 & 0.26 & 0.73 & 1.09 \\
\hline \multicolumn{5}{|c|}{ Without $N$-saturation } \\
\hline IMAGES & 0.04 & 0.12 & 0.57 & 0.73 \\
\hline ECHAM & 0.05 & 0.19 & 0.73 & 0.98 \\
\hline GCTM & 0.07 & 0.15 & 0.73 & 0.95 \\
\hline GRANTOUR & 0.04 & 0.12 & 0.50 & 0.66 \\
\hline MOGUNTIA & 0.06 & 0.20 & 0.64 & 0.90 \\
\hline MOGUNTIA $\mathrm{NH}_{x}+\mathrm{NO}_{y}$ & 0.11 & 0.29 & 1.02 & 1.42 \\
\hline
\end{tabular}

${ }^{\mathrm{l}}$ Based on $\mathrm{CO}_{2}$ concentrations

${ }^{2}$ Based on $\delta^{13} \mathrm{C}$ and $\mathrm{CO}_{2}$ concentrations

${ }^{3}$ All values are negative (net sinks).

\subsection{Factors Limiting Ecosystem Response to N Additions}

The carbon uptake of ecosystems receiving large and chronic amounts of nitrogen from the atmosphere has the potential to gradually become unresponsive to $\mathrm{N}$ deposition, thus breaking the link between the $C$ and $N$ cycles (Asner et al. In press). Further, some ecosystems will even begin to lose stored carbon. This removal of ecosystem $\mathrm{N}$-limitation, though a 
cumulative effect, can manifest itself relatively suddenly and be associated initially with losses of nitrogen from the system via leaching and denitrification (Aber et al. 1989, Stoddard 1994). Eventually, ecosystem productivity will decline and, in the worst cases, will be devastated. This process is often referred to as nitrogen saturation (Aber et al. 1989. Schulze 1989), though other pollutant species are usually involved, and interactions leading to this state are complex and highly dependent on local conditions (Mälkōnen 1990, Zöttl 1990, Gundersen 1991). In this section, we examine the factors leading to mitigation or removal of nitrogen fertilization and their parameterization in a simple model like NDEP.

\subsubsection{Deleterious effects associated with chronic nitrogen deposition}

It is well known that nitrogen deposition is not only a fertilizing agent for terrestrial vegetation but. depending on the source, will be associated with atmospheric pollutants, and can itself be damaging in large quantities. The atmospheric pollutants associated with industrial and agricultural activities that have the potential to damage ecosystems are heavy metals. gaseous oxidant species (particularly ozone), and acid species (Aber et al. 1989, McLaughlin and Downing 1995, Reich 1987). Impacts on ecosystem function due to pollutant deposition. though chronic in time, are an acute spatial phenomenon. They have been most notably observed in forests nearby and downwind of major urban-agricultural complexes (Chameides et al. 1994). In the northeastern US, and in parts of Europe, deposition of nitrogen and sulfur may be as much, or more than 100 times greater than "natural" levels. Generally, the deposition is due to a combination of agricultural and industrial sources of $\mathrm{NO}_{\mathrm{y}}$ and $\mathrm{NH}_{\mathrm{z}}$, except in some highly impacted regions of the Netherlands where ecosystems proximate to very large feedlots receive mostly ammonium. 


\section{Acidification}

Oxidation of the primary pollutants $\mathrm{NO}_{x}$ and $\mathrm{SO}_{2}$ by $\mathrm{OH}$ lead to the production of $\mathrm{H}_{2} \mathrm{SO}_{4}$ and $\mathrm{HNO}_{3}$ in the atmosphere which are eventually deposited in precipitation or as aerosols. These acids tend to dissociate fully so that they are associated with the introduction of $\mathrm{SO}_{4}{ }^{2-}, \mathrm{NO}_{3}{ }^{-}$and $\mathrm{H}^{+}$into ecosystems, thus contributing to a lower $\mathrm{pH}$ in the soil environment. Another toxifying mechanism is associated with the release of ammonia $\left(\mathrm{NH}_{3}\right)$ from agricultural and livestock-bearing lands. Nitrification of subsequently deposited $\mathrm{NH}_{\mathbf{x}}$ can lead to increased acidity in soils because of the high mobility of nitrate relative to ammonium. Further, there exist mechanisms through which ecosystem health is affected by nutrient imbalances that result from increased availability of mineral nitrogen; these problems potentially interact with acidification and other pollutant effects.

The toxic effects of acidification are primarily related to altered soil chemistry. Inputs of $\mathrm{H}^{+}$increase the rate of weathering of soil minerals, and mobilize cations (most notably $\mathrm{Al}^{3+}$ ) from exchange sites into the soil solution. In some forest soils, gibbsite is weathered more rapidly, releasing $\mathrm{Al}^{3+}$, which is known to inhibit plant growth at high levels. In addition, important base cations may be lost from the soil due to their increased mobility (lower cation exchange capacity) at low $\mathrm{pH}$. Depending on the parent rock material, the lost cations will usually be one of the following essential nutrients: $\mathrm{K}^{+}, \mathrm{Ca}^{2+}$, or $\mathrm{Mg}^{2+}$. These two effects together are especially detrimental because plant uptake of cation nutrients may be suppressed by excess soil aluminum. Godbold et al. (1988) found that aluminum toxicity in German forests receiving high deposition was related to interference with root elongation and uptake of $\mathrm{Ca}$ and Mg. They speculated that this mechanism leads to leaf imbalances and is the primary mode of damage from acidification. 


\section{Nitrogen saturation}

Another deleterious effect that is associated with pollutant deposition is the literal saturation of ecosystems with respect to nitrogen (Aber et al. 1989). Nitrogen saturation refers to ecosystem decline resulting from mineral $\mathrm{N}$ availability that is in chronic and extreme excess of plant and microbial demand. There are three hypothetical stages in this process (Aber et al. 1989): Initially the $\mathrm{N}$-limited ecosystem responds by increasing plant and microbial uptake, reflected by increases in NPP and N mineralization (stage 1). In the second stage (saturation), excess $\mathrm{NH}_{4}{ }^{+}$is rapidly nitrified, followed by losses due to denitrification and nitrate leaching. By the end of this stage, inputs to the ecosystem will roughly equal outputs $\left(\mathrm{N}_{2} \mathrm{O}\right.$ and $\mathrm{N}_{2}$ from denitrification plus $\mathrm{NO}_{3}{ }^{-}$leaching). Meanwhile, the plant community is faced with a unique and non-adaptive stress: excess soil $\mathrm{N}$ will lead to increased uptake and losses of fine root mass and mycorrhizal infection, which in turn will cause insufficient levels of other nutrients or nutrient imbalances within the plant. It is also possible that foliage $\mathrm{NH}_{4}{ }^{+}$may reach levels that are toxic to metabolic functions. Both these factors will signal the onset of forest decline (stage 3).

\section{Ozone exposure}

Tropospheric ozone is probably the most harmful pollutant to terrestrial vegetation, interfering with many physiological processes including photosynthesis, respiration, and allocation (Chameides et al. 1994, Reich 1987). The net effect is a reduction in growth. Because nitrogen oxides are precursors to tropospheric ozone formation, surface ozone concentrations are correlated with $\mathrm{NO}_{\mathrm{y}}$ deposition, as demonstrated using results from the IMAGES model (Fig. 3.14; Section 3.3). Thus $\mathrm{O}_{3}$ can limit the carbon storage to $\mathrm{N}$ fertilization at high levels of deposition. Both surface $\mathrm{O}_{3}$ mixing ratios and $\mathrm{NO}_{\mathrm{y}}$ deposition (in Fig. 3.14) are annual averages over large grids and are underestimated with respect to intensely 


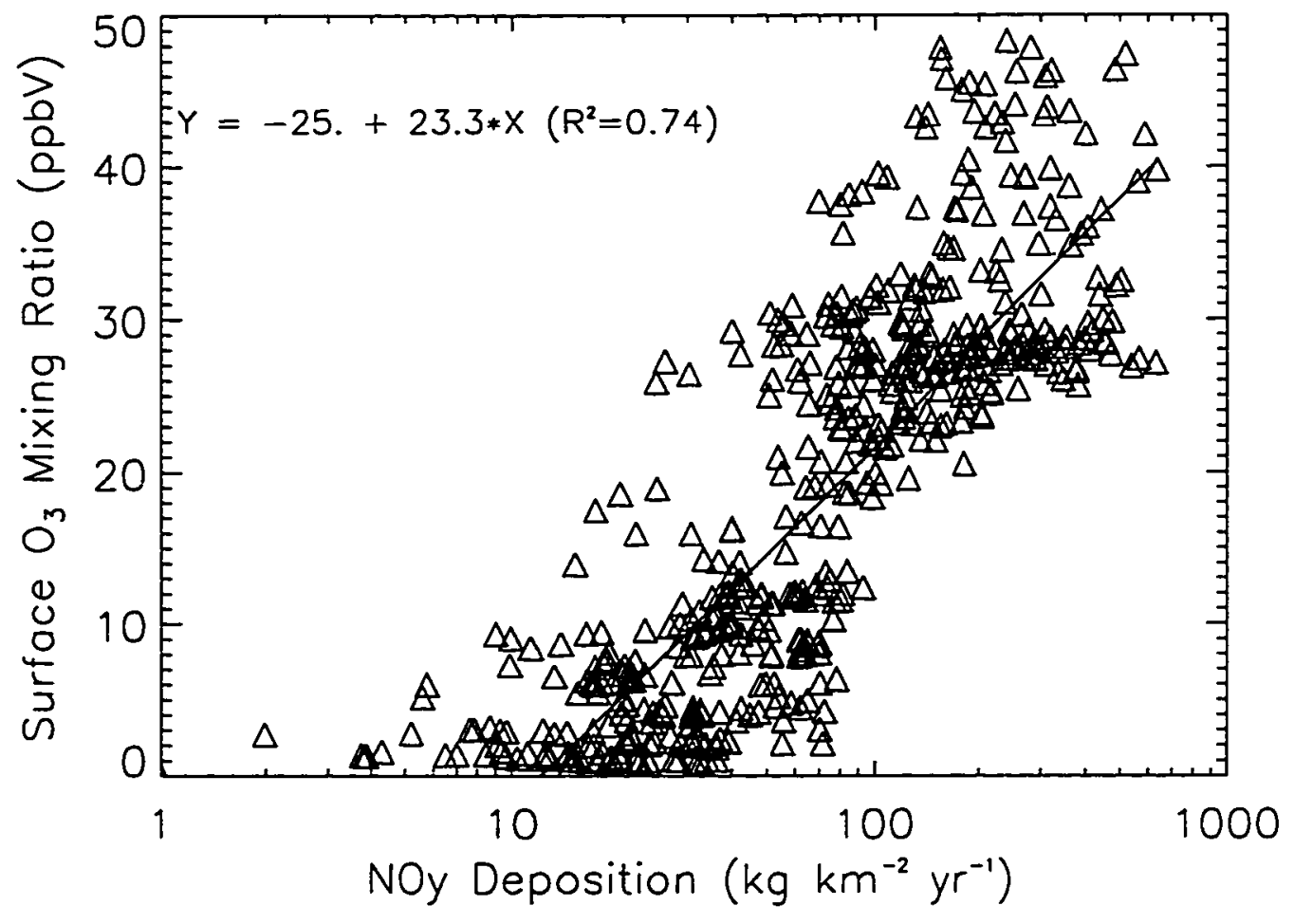

Figure 3.14 Annual average ozone concentration from the surface layer of IMAGES $(900 \mathrm{mb})$ compared to the predicted $\mathrm{NO}_{\mathrm{y}}$ deposition $\left(\mathrm{kg} \mathrm{N} \mathrm{km}^{-2} \mathrm{yr}^{-1}\right)$ including both wet and dry deposition of $\mathrm{N}$. The comparison was limited to those grid cells containing natural vegetation. 
localized spatial and temporal exposure, which is most common (Müller and Brasseur 1995, Reich 1987). This results in few $\mathrm{O}_{3}$ concentrations above the critical threshold for plant damage of 50-70 ppbv (Reich 1987).

Though both spatial and temporal averaging may contribute to the underestimation of impacts, the correlation between $\mathrm{O}_{3}$ concentration and $\mathrm{NO}_{\mathrm{y}}$ deposition (Fig. 3.14) suggests that the chemical feedbacks represented by the individual and interactive mechanisms of acid deposition, ozone damage, and alleviation of $\mathrm{N}$-limitation have the potential to be represented collectively. Nevertheless, the underlying mechanisms deserve further attention, with respect to both atmospheric and ecosystem processes. For example, a more realistic parameterization might involve specifying the partitioning of $\mathrm{N}$ deposition with respect to $\mathrm{NH}_{\mathrm{x}}$ and $\mathrm{NO}_{\mathrm{y}}$ as these are utilized somewhat differently by ecosystems. Also, model-generated $\mathrm{O}_{3}$ concentration might be useful (given fine enough spatial and temporal resolution) because its effect is only important at relatively high levels of exposure. In the NDEP model, we implemented a rudimentary parameterization of the net effect of these mechanisms (Section 3.2 and below).

\subsubsection{Representing the approach to saturation in NDEP}

In our previous estimates of global terrestrial fertilization by anthropogenic $\mathrm{N}$ deposition (Townsend et al. 1996, Holland et al. In press; Section 3.2 and 3.3), we recognized that the most highly impacted regions (downwind from urban, industrial, or agricultural centers) eventually cease being net $C$ sinks, and could actually become $C$ sources as excess carbon stored in soils and wood is gradually released. Thus we compared our baseline estimates which assume continuing $\mathrm{N}$-limitation to simulations that include a linearly declining ecosystem $\mathrm{N}$ retention function (Fig. 3.15). Loss of $\mathrm{N}$ varied from $20 \%$ of the available $\mathrm{N}$ flux $\left(N_{\min }+N_{\text {dep }}\right.$; see Equation 3.9) to $100 \%$ at a threshold value of total $\mathrm{N}$ deposition $\left(N_{\text {crit }}\right)$ of 2000 


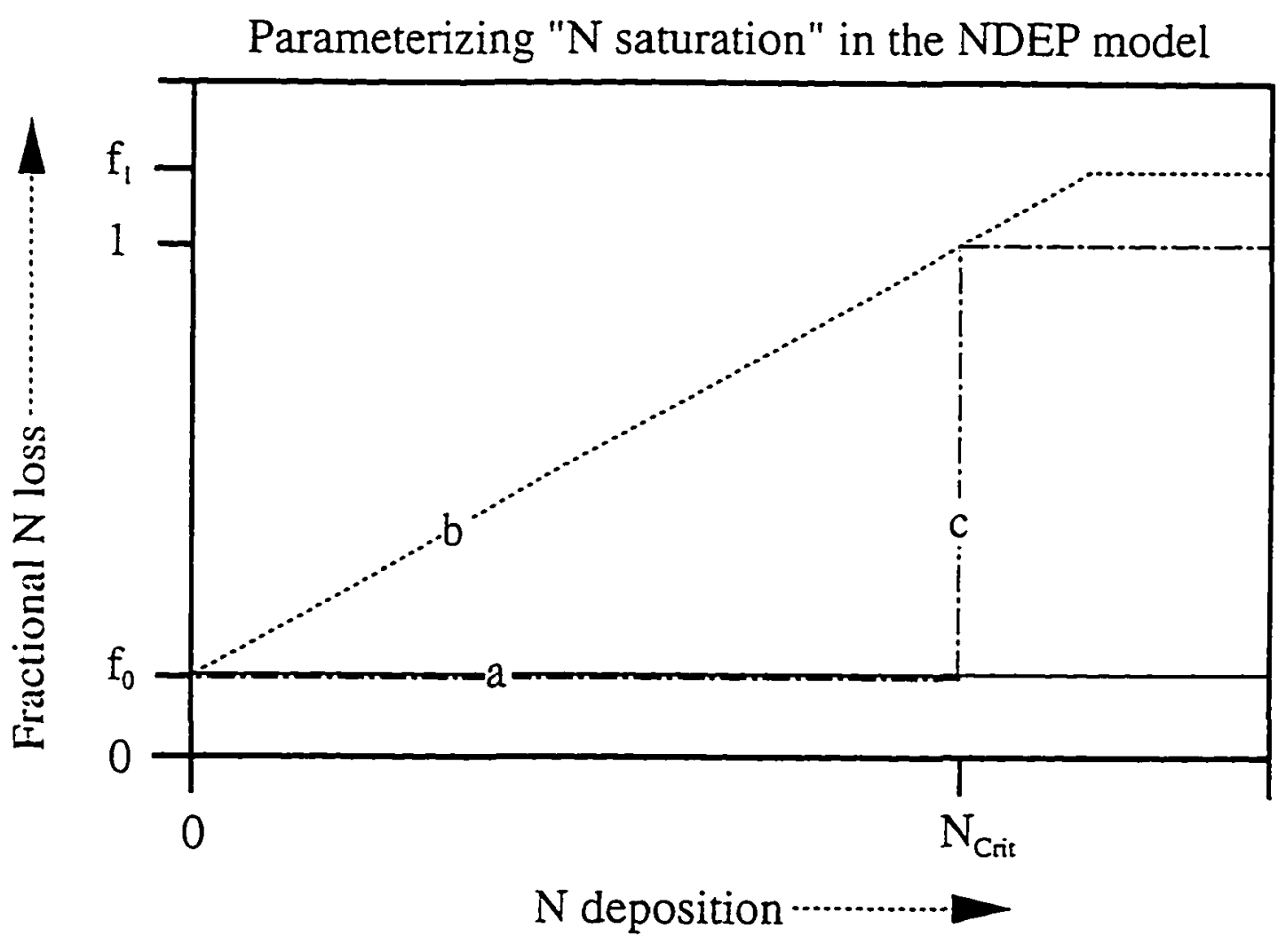

Figure 3.15 Schematic representing the parameterization of nitrogen saturation in the NDEP model. Ecosystem retention of available $\mathrm{N}$ is assumed to be a function of deposition. Curve $a$ represents the base case where there are no feedbacks affecting $N_{\text {loss }}\left(f_{0}=0.8\right.$ in all calculations); curve $b$ represents a linearly declining ecosystem use efficiency up to the point where the system is unresponsive; curve $c$ represents the extreme case of a sudden onset of saturation which was not implemented in Section 3.2 (Townsend et al. 1996) or Section 3.3 (Holland et al. In press), but is discussed in Section 3.4. 
$\mathrm{kg} \mathrm{N} \mathrm{km}^{-2} \mathrm{yr}^{-1}$ at which inputs equal losses. Deposition rates higher than $N_{\text {crit }}$ (which did not occur in either study) would be associated with net loss of excess carbon accumulated during the period of fertilization. When considering the sink due to $\mathrm{NO}_{\mathrm{y}}$ or $\mathrm{NH}_{\mathrm{x}}$ alone, we set the threshold to be $1000 \mathrm{~kg} \mathrm{~N} \mathrm{~km}^{-2} \mathrm{yr}^{-1}$ under the assumption that each contributes roughly one half of total $\mathrm{N}$ deposited.

It is interesting to note how the ecosystem "excess" $N$ and $C$ fluxes and cumulative storage change in time for an individual grid cell. Figs. 3.16 and 3.17 show the time trajectories of flux and storage, respectively. for the single MOGUNTIA (Dentener and Crutzen 1994; Section 3.3) grid cell, located in northern Europe, that received the most total nitrogen input. For this and the few similarly impacted grid cells, ecosystem-recycled nitrogen is not very important; the sink is driven almost entirely by deposition. Globally, however, recycled $\mathrm{N}$ is responsible for $\sim 10 \%$ of the $\mathrm{C}$ sink estimate, and the inclusion of multiple dynamic compartments yields an estimate that is $-30 \%$ higher than without recycling primarily because of the narrow $\mathrm{C}: \mathrm{N}$ ratios of stored SOM pools relative to vegetation.

We looked closer at the $\mathrm{N}$-saturation parameterization by including two new simulations (Table 3.6) based on the high and low MOGUNTIA estimates of total anthropogenic $\mathrm{N}$ deposition (Section 3.3). First, we tested the sensitivity to the threshold value $N_{\text {crit }}$ for saturation by lowering it from $2000 \mathrm{~kg} \mathrm{~N} \mathrm{~km}^{-2} \mathrm{yr}^{-1}$ to $1000 \mathrm{~kg} \mathrm{~N} \mathrm{~km}^{-2} \mathrm{yr}^{-1}$ and using the linear function (Figs. 3.16c and 3.17c). The $\mathrm{N}$ deposition load at which inputs equal outputs, being a convenient parameter for our model, is highly variable and the result of complex interactions. Schulze et al. (1989) indicate that the "critical load" (defined similarly as $N_{\text {crii }}$ ) for $\mathrm{N}$ deposition on ecosystems can vary from $300-4800 \mathrm{~kg} \mathrm{~km}^{-2} \mathrm{yr}^{-1}$, depending on the parent soil material and the quantity of deposition of accompanying anions (e.g., $\mathrm{SO}_{4}{ }^{2}$ ). Our reason for selecting a lower value was to test the realism of the model when $N_{\text {dep }}>N_{\text {crit }}$; the 
Table 3.6 Summary results of NDEP simulations using high and low estimates of total anthropogenic $\mathbf{N}$ deposition from MOGUNTIA for various parameterizations of $\mathrm{N}$ saturation feedbacks. The high and low estimates incorporate various proportions of the $\mathrm{NH}_{\mathrm{x}}$ and nonfossil $\mathrm{NO}_{\mathrm{y}}$ emissions (see text).

\begin{tabular}{lcc}
\hline N saturation parameterization & $\begin{array}{c}\text { Low C sink estimate } \\
\left(\mathrm{Pg} \mathrm{yr}^{-1}\right)\end{array}$ & $\begin{array}{c}\text { High C sink estimate } \\
\left(\mathrm{Pg} \mathrm{yr}^{-1}\right)\end{array}$ \\
\hline Constant $N_{\text {loss }}$ & 1.42 & 1.97 \\
Linear $N_{\text {loss }} ;$ & 1.09 & 1.35 \\
$N_{\text {cru }}=2000 \mathrm{~kg} \mathrm{~km}^{-2} \mathrm{yr}^{-1}$ & 0.75 & 0.73 \\
Linear $N_{\text {losss }} ;$ & & \\
$N_{\text {crit }}=1000 \mathrm{~kg} \mathrm{~km}^{-2} \mathrm{yr}^{-1}$ & 1.30 & 1.59 \\
Step $N_{\text {loss }} ;$ & & \\
$N_{\text {crit }}=1000 \mathrm{~kg} \mathrm{~km}^{-2} \mathrm{yr}^{-1}$ & &
\end{tabular}

highest MOGUNTIA total $\mathrm{N}$ deposition is approximately $2000 \mathrm{~kg} \mathrm{~N} \mathrm{~km}^{-2} \mathrm{yr}^{-1}$.

Interestingly, the "low" deposition case (Table 3.6) resulted in a slightly greater sink than the "high" deposition case because of the massive loss of $C$ due to over-saturated grid cells (Fig. 3.16c). The most heavily impacted systems lose back all of the enhanced storage of carbon accumulated over more than one hundred years in just about thirty years. This dynamic, while it may be representative of the "decline" phase of N-saturation (Aber et al. 1989), is difficult to validate because of the distinction made here with respect to natural vs. anthropogenic nitrogen. Nevertheless, whole-watershed nitrogen budgets for ecosystems receiving heavy loads of atmospheric deposition would be helpful for understanding the net, large scale effects of saturation before and after the time when $N_{\text {dep }}=N_{\text {crit }}$.

The final simulation utilized a different function: a near-instantaneous step from $20 \%$ $\mathrm{yr}^{-1}$ losses to $N_{\text {loss }}$ equal to $100 \%$ of $N_{\text {dep }}$ (Fig. 3.15; Figs. 3.16d and 3.17d). The step function parameterization was designed to test the extreme case of the sudden onset of saturation. Studies of watersheds in the US that receive high $\mathrm{N}$ deposition (Stoddard 1994), and modeling studies (e.g., Aber et al. In press) indicate that ecosystem $\mathrm{N}$ losses do not increase (and 

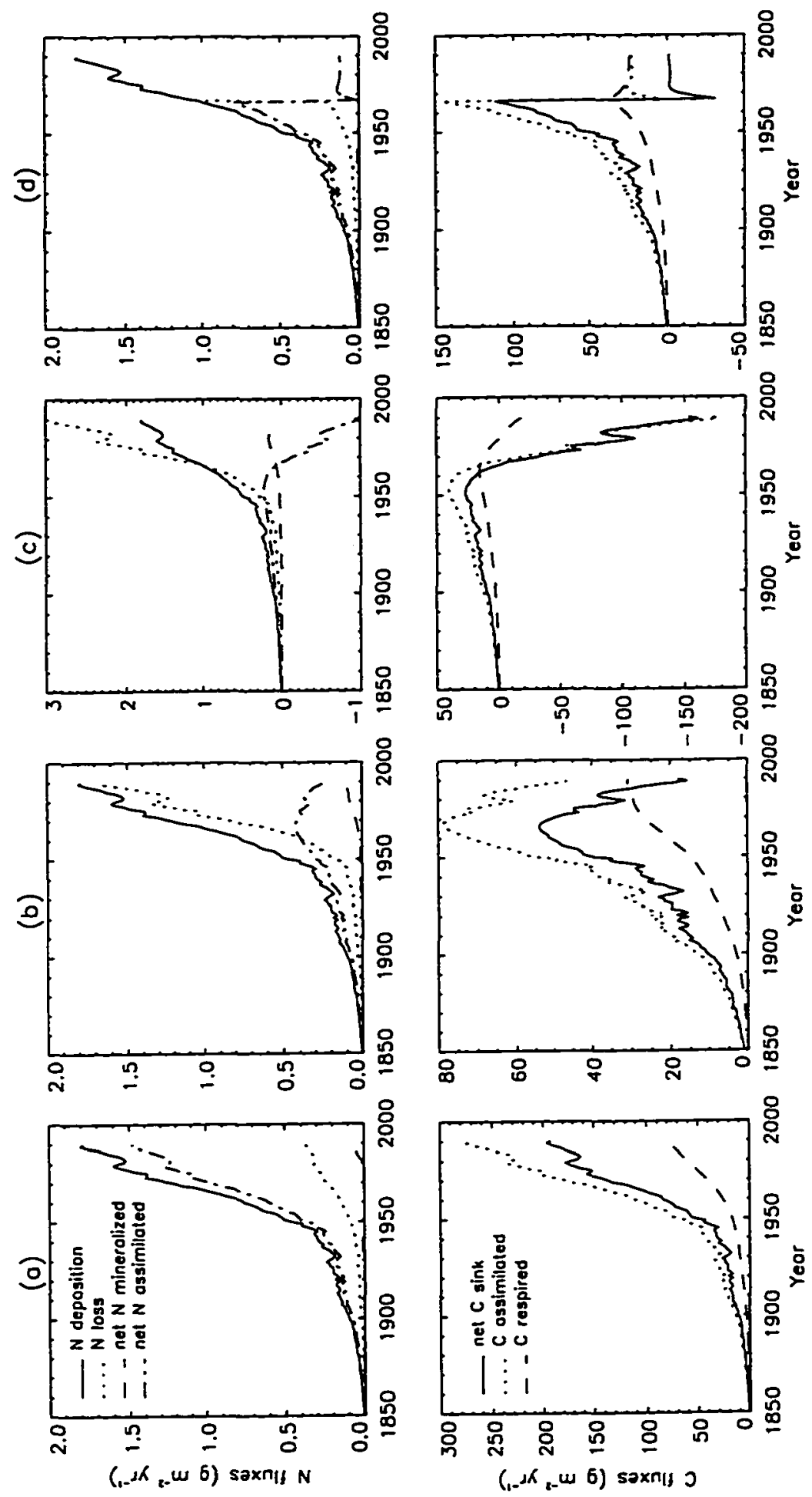

Figure 3.16 Time series of $\mathrm{N}$ and $\mathrm{C}$ fluxes for one forest grid cell under various assumptions about changing ecosystem $\mathrm{N}$-limitation. (a) The base case, corresponding to curve $a$ in Fig. 3.15; (b) Linear approach to $\mathrm{N}$ saturation (curve $b$ in Fig. 3.15), with a critical value $N_{\text {crit }}=2.0$ $\mathrm{g} \mathrm{m}^{-2} \mathrm{yr}^{-1}$; (c) Linear function, as in (b), but with a critical threshold of $1.0 \mathrm{~g} \mathrm{~m}^{-2} \mathrm{yr}^{-1}:$ (d) $\mathrm{A}$ sudden onset of $\mathrm{N}$ saturation. corresponding to curve $c$ in Fig. 3.15 , with $N_{\text {crit }}=1.0 \mathrm{~g} \mathrm{~m}^{-2} \mathrm{yr}^{-1}$. 

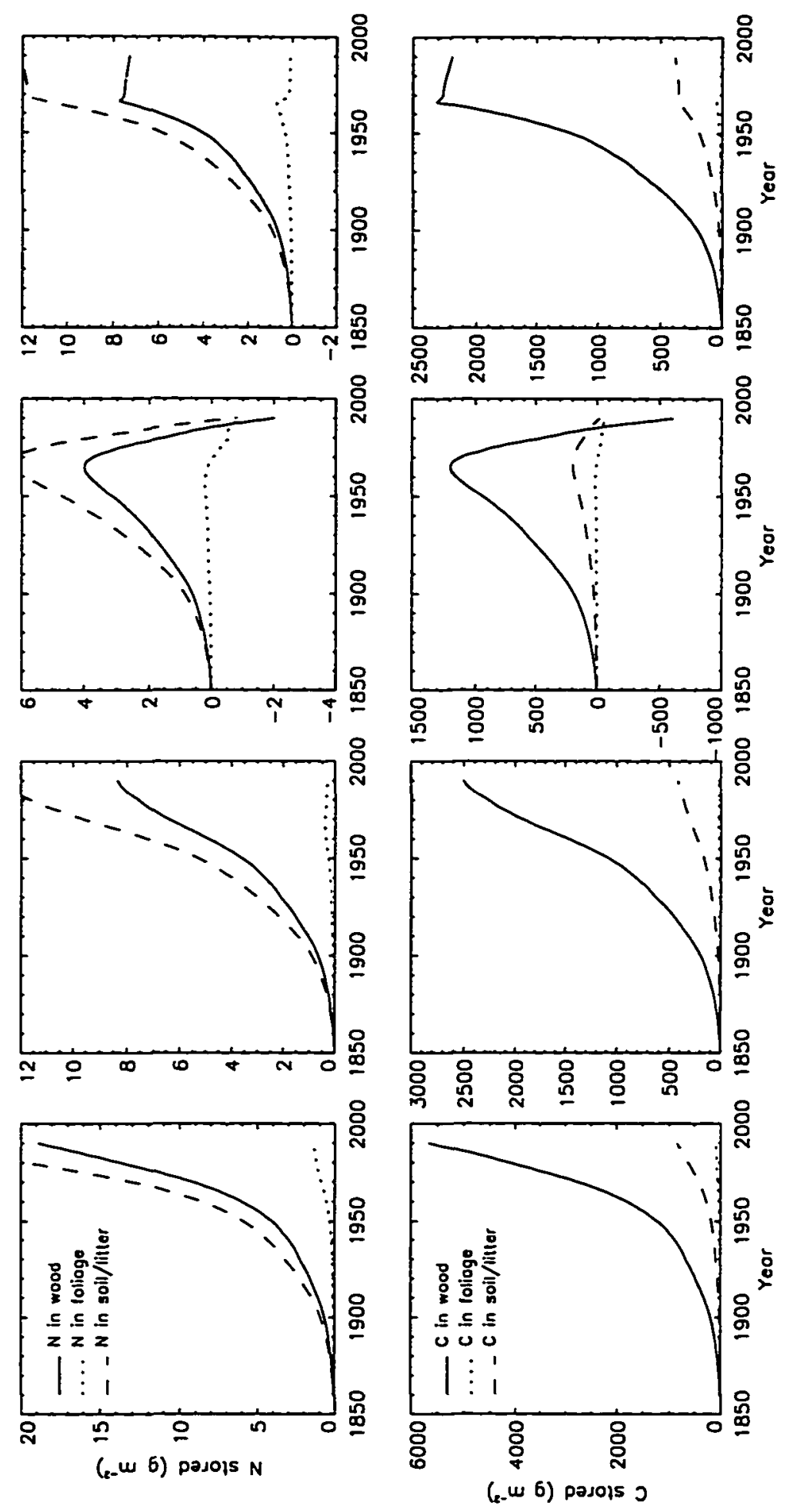

Figure 3.17 Time series of accumulated $N$ and $C$ in wood, foliage, and total soil/litter poois for the same simulations as in Fig. 3.16. Note that in the most extreme cases, the system begins to lose the excess anthropogenic carbon and nitrogen it has stored over the years. 
presumably vegetation productivity does not decline) immediately with enhanced deposition, as modeled by our linear function. Rather, the onset of massive leakage of $\mathrm{N}$ to the environment can be somewhat sudden and associated with increased nitrification (leading to losses of $\mathrm{NO}_{3}{ }^{-}$in streamflow) and denitrification (leading to losses of $\mathrm{N}_{2}$ and $\mathrm{N}_{2} \mathrm{O}$ ). Thus the linear and step functions effectively bracket the problem, except to the extent that the actual $N_{\text {loss }}$ fraction might be highly variable at the ecosystem scale. Considering the uncertainty of $N_{c r i t}$ and the timing of $\mathrm{N}$ saturation as parameterized in these four simulations (Table 3.6), our estimate of the total current $C$ sink due to anthropogenic nitrogen emissions is thus revised to the range $0.73-1.97 \mathrm{Pg} \mathrm{yr}^{-1}$.

\subsection{Summary}

Because the global cycles of carbon and nitrogen are linked by the biosphere, understanding the present and future exchange of carbon between the terrestrial land surface and the atmosphere requires understanding critical interactions involving nitrogen. We described in Section 3.2 (also Townsend et al. 1996) an estimate of terrestrial carbon storage arising from deposition of fossil fuel derived nitrogen that accounts for spatial distributions in deposition and vegetation types, turnover of plant and soil carbon pools, and the cumulative effects of deposition. The overlying templates of deposition and vegetation type have a pronounced effect on carbon uptake; the combination of high $\mathrm{C}: \mathrm{N}$ ratios and long lifetimes in wood may create a significant sink in forests, but much of the nitrogen falls on cultivated areas and grasslands, where there is limited capacity for long-term carbon storage. Because of this potential for strong spatial dependence, we further examined the sensitivity of the carbon sink to spatial patterns of deposition as predicted by various chemistry-transport models (Holland et al. In press; Section 3.3). 
Unlike carbon dioxide, most anthropogenically produced nitrogen species are reactive in the atmosphere and undergo complex transformations before they are deposited or converted to unreactive forms (e.g., $\mathrm{N}_{2}$ and $\mathrm{N}_{2} \mathrm{O}$ ). Thus, physical and chemical processes that govern the spatial distribution of deposition must be adequately represented. We found that differences in the structure and parameterization of the atmospheric chemistry and transport models led to significantly different patterns of predicted $\mathrm{NO}_{\mathrm{y}}$ deposition on land. These contrasting predictions propagated into differences in enhanced net terrestrial carbon exchange modeled by

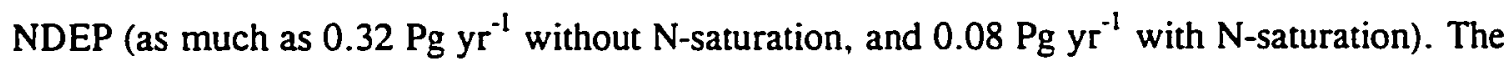
differences in deposition were small, however, compared to the discrepancy between modeled and observed $\mathrm{NO}_{\mathrm{y}}$ deposition at the continental scale (inferred from NADP/NTN wet $\mathrm{NO}_{\mathrm{y}}$ observations for the US; Fig. 3.6). Further, the importance of ammonia chemistry, transport, and deposition were not insignificant as we initially asserted (Townsend et al. 1996; Section 3.2). Therefore, for the purposes of studying the impacts of atmospheric $\mathrm{N}$ on terrestrial ecosystems, inclusion of $\mathrm{NH}_{\mathrm{x}}$ is critical. Further, careful attention must be paid to dry deposition and rainout parameterizations which determine in large part the regional patterns of deposition (Fig. 3.6).

Closing the global $\mathrm{N}$ cycle is important for clearly depicting the relationship between global biogeochemistry and climate. Terrestrial ecosystems are a source of reactive $\mathrm{N}$ species to the atmosphere, as well as a sink. A portion of terrestrial $\mathrm{N}$ sources are due to anthropogenic activity (i.e., those arising from land-use/cover change) that is highly variable in space and time. Thus, land use practices, which impact trace gas emissions as well as the details of terrestrial biogeochemical cycling are important factors in the bio-atmospheric cycling of nitrogen. A better understanding of the global interactive $\mathrm{C}$ and $\mathrm{N}$ cycles will result from more realistic models of atmospheric chemistry and transport, validation of terrestrial processes that 
govern the assimilation, allocation, and turnover of $\mathrm{C}$ and $\mathrm{N}$ in ecosystems, emissions of gaseous $\mathrm{N}$ species, and the interaction of mechanisms that control net exchange (e.g., climate and atmospheric $\left.\left[\mathrm{CO}_{2}\right]\right)$.

We calculated a carbon sink due to total anthropogenic $\mathrm{N}$ emissions (0.73-1.97 $\mathrm{Pg} \mathrm{yr}^{-1}$ ) that is significant on the scale of the global carbon budget (Schimel et al. 1994, Schimel 1995b; Chapter 2), and potentially accounts for as much as half of the residual in the "highconfidence" carbon budget (Table 2.1, column 1). The near-convergence of $\mathrm{C}$ sink estimates when any parameterization of saturation is used (with any CTM deposition prediction) suggests that one key to providing accurate estimates of enhanced $C$ uptake will be knowledge of the dependence of $N_{\text {crit }}$ (Section 3.4) on land cover type, land-use history (Aber et al. In press), deposition rate, and perhaps $\mathrm{O}_{3}$ concentrations. It is clear that there are interactions with other atmospheric pollutants (primarily ozone and sulfate) and internal $\mathbf{N}$ cycling dynamics that can lead to the mitigation of this sink over large areas.

Whatever the magnitude of the sink due to human-derived nitrogen fluxes, we expect it will not continue to increase steadily. Interannual variability in net terrestrial-atmosphere $\mathrm{CO}_{2}$ fluxes are on the order of $1-2 \mathrm{Pg} \mathrm{C} \mathrm{yr}{ }^{-1}$ (Keeling et al. 1989a; Chapter 4). Superimposed on this signal is a net terrestrial sink of approximately the same magnitude (Ciais et al. 1995,a,b Keeling et al. 1995) arising from a number of mechanisms, including $\mathrm{N}$-fertilization (see also Chapter 2). Many of these mechanisms $\left(\mathrm{CO}_{2}\right.$ and $\mathrm{N}$ fertilization, and temperate forest regrowth) are intrinsically limited with respect to the amount of carbon they can continue to sequester in the future because of negative feedbacks operating at various scales (e.g., $\mathrm{N}$ saturation; Section 3.4). It is therefore reasonable to assert that as these mechanisms approach an asymptote, the growth rate of atmospheric $\mathrm{CO}_{2}$ concentrations will experience a corresponding increase. 


\section{CHAPTER 4}

\section{EQUILIBRIUM AND TRANSIENT SYSTEM-LEVEL RESPONSE OF TERRESTRIAL BIOGEOCHEMICAL DYNAMICS: MODELS AND OBSERVATIONS}

\subsection{Introduction}

Understanding how ecosystems respond to climate over large spatial scales is important for improving our ability to predict patterns of biosphere-atmosphere exchange of carbon, energy, and water. Similarly, it is important to know how ecosystem processes (e.g., plant production and soil organic matter decomposition) respond dynamically to multiple resource limitation. This chapter explores issues surrounding the interactive and competing influences of abiotic (climatic and edaphic) and biotic forces that act to determine ecosystem behavior. In particular, we focus on the factors that influence terrestrial net primary productivity (NPP) and net ecosystem production (NEP), i.e. gross and net carbon exchange with the atmosphere.

Two principal resources that can be limiting to plants and microbes are mineral nutrients and water. For the terrestrial biosphere, the most common limiting nutrient is nitrogen (Vitousek and Howarth 1991). Though external inputs of nitrogen (through wet and dry deposition) can be important (Chapter 3), the primary fluxes of usable $\mathrm{N}$ are biologically mediated and internally recycled. Water is a resource that is associated with the physical environment (precipitation), but the amount of available water in an ecosystem is regulated in part by vegetation (transpiration). Variability, in both space and time, of plant and microbial response to limiting water and nutrients is controlled by patterns of biological resource-use efficiencies and patterns of climate. 
Ambient temperature is an important climatic variable, unique in that it is relatively decoupled from (i.e., not strongly mediated by) biological activity. In this chapter we use temperature as a primary independent variable with which to probe the behavior of ecosystem biogeochemistry, both in the approach to "steady-state" and in a transient mode. While it is clear that changing climate (e.g., temperature) influences biogeographic patterns of ecosystems, we focus in this chapter on biogeochemical effects both within and between biome types, for the current distribution of potential vegetation (i.e., land use is neglected). Adjustment of biome boundaries associated with changing climate, and redistribution of vegetation by anthropogenic land-use/cover change potentially alter conclusions about terrestrial biosphereatmosphere interactions. However, we wish to first study the activity of natural vegetation before looking at other anthropogenic interactions.

Spatially resolved terrestrial biogeochemical models provide a means to link theory describing soil organic matter dynamics, litter decomposition, and plant growth and turnover to geographically referenced databases of soil and vegetation properties and climate. These models typically describe rates of change in carbon, water, and nutrient stocks as a function environmental conditions, and the state of the components themselves (McGuire et al. 1992. Parton et al. 1993, Running and Hunt 1993). Symbolically this can be expressed as:

$$
\frac{d \mathbf{S}(\mathbf{x}, t)}{d t}=\mathbf{f}\left(\mathbf{S} ; \mathbf{C}(\mathbf{x}, t) ; \mathbf{P}_{V}(\mathbf{x}) ; \mathbf{P}_{S}(\mathbf{x})\right)
$$

where $\mathbf{S}=\left(S_{1}, S_{2}, \ldots, S_{n}\right)^{\mathrm{T}}$ is the vector of all state variables, $\mathbf{f}$ is the set of rules for transformation of $\mathbf{S}, \mathbf{C}(\mathbf{x}, t)$ is the field of meteorological input variables, and $\mathbf{P}_{V}(\mathbf{x})$ and $\mathbf{P}_{S}(\mathbf{x})$ are the spatial templates of vegetation and soil-specific parameters. Most biogeochemical 
models are not strictly implemented as an integration of a set of coupled differential equations because of the use of discontinuous functions ("if - then" rules), but in principal they can be represented by (4.1).

Models have the potential to be used to make predictions where no ecological data are available (i.e., knowledge-based interpolation), and to investigate the importance of multiple processes in determining large-scale ecosystem fluxes. Validation of continental-to-global scale model simulations is difficult, however, because of the great deal of heterogeneity of ecological patterns and boundary conditions at the local scale where the bulk of observations are made. Thus, model intercomparison is the first step toward checking the realism of the simulations (e.g., VEMAP 1995). To the extent that conclusions are made about large-scale terrestrial biogeochemistry using models, the observations that are most directly comparable (having commensurate spatial resolution) are atmospheric measurements of $\mathrm{CO}_{2}$ and its isotopes (see Chapter 2) and moderate resolution remote sensing (see Chapter 5). We employ both types of observations in this chapter.

In the following section (4.2) we use the Century terrestrial ecosystem model (Parton et al. 1987,1993$)$ to evaluate the relative roles of water and nitrogen limitation of net primary productivity, both spatially and in response to climate variability. We focused on the three principal fluxes of carbon, water, and nitrogen, respectively: NPP, evapotranspiration (ET), and nitrogen mineralization (NMIN). We performed/analyzed three model experiments. First, we studied the spatial relationship between NPP, ET, and NMIN for a global quasi-steady state simulation using dynamic maps of current climate and an assumed distribution of potential vegetation (Cramer et al. 1995). Second, we investigated the effect of temperature on these relationships by performing a temperature perturbation experiment for a network of model 
validation sites. Third, we used decadal-scale temperature records (estimated from satellite) for the same sites and observed the modeled transient response of ecosystems to climate.

Observational data from satellites and an atmospheric monitoring network were also used (Section 4.3) to check for consistency with the interpretation of the Century model experiments. We performed simple statistical analyses on globally and hemispherically averaged atmospheric $\mathrm{CO}_{2}$ concentrations, temperature, and vegetation "greenness" (a remotely sensed vegetation index; see Chapter 5). Additionally, more detailed statistical analyses were performed on the spatial-temporal satellite data (temperature and vegetation index) because of the likelihood that individualistic ecosystem responses and spatially localized interactions complicate the signal at the global or hemispheric level. We introduce a unique and indirect way to compare modeling and observational evidence regarding characteristic response times of terrestrial systems to climate perturbations, and the implications for atmospheric $\mathrm{CO}_{2}$.

\subsection{Climate and Nitrogen Controls on the Geography and Time Scales of Terrestrial Biogeochemical Cycling ${ }^{\dagger}$}

\subsubsection{Introduction}

Terrestrial ecosystem models have progressed from early, highly aggregated models to include mechanistic or parametric representations of the ecological, hydrological, and biogeochemical processes that dynamically control carbon storage and net primary productivity (NPP) (Melillo et al. 1993, Potter et al. 1993, Schimel et al. 1994). Process-based models for terrestrial biogeochemistry are important for furthering ecosystem science because they allow the extrapolation of fluxes to large spatial scales, as well as the comparison of model predictions to the growing body of global observations. Whereas most extant ecological data

\footnotetext{
${ }^{\dagger}$ Adapted from the article by D. S. Schimel, B. H. Braswell, R. McKeown, D. S. Ojima, W. J. Parton, and W. Pulliam, Global Biogeochemical Cycles, In press. Copyright by the American Geophysical Union.
} 
are site- or system-specific (in contrast to the standardized sectional or global sampling widely used in meteorology and oceanography for seawater $\left[\mathrm{CO}_{2}\right]$, atmospheric $\mathrm{CO}_{2},{ }^{13} \mathrm{CO}_{2}$, and physical variables), collaborative activities such as the International Geosphere-Biosphere Program (IGBP) and new satellite techniques are resulting in improved global ecosystem data. Spatially-resolved process models provide insights into large-scale controls and provide a consistent framework for comparisons with existing observations.

Spatial ecosystem models are derived from theory linking climate, soil properties, and species- or growth form-specific traits to biogeochemical responses of plants and microorganisms (Farquhar et al. 1980, Melillo et al. 1984, Bloom et al. 1985, Chapin et al. 1987. Nobel 1991, Running and Nemani 1991). As discussed in Chapter 3, many studies have demonstrated nutrient limitation of terrestrial primary productivity (i.e., added nutrients lead to additional plant growth and carbon storage) (Vitousek and Howarth 1991, Schimel 1995b); however, large-scale patterns in terrestrial primary productivity, soil carbon, and soil metabolism can often be explained from simple equations using climate parameters (precipitation, actual evapotranspiration, solar radiation) (Leith 1975, Post et al. 1985. Uchijima and Seino 1985, Sala et al. 1988. Potter et al. 1993, Gifford 1994. Zak et al. 1994). Within grasslands, empirical climate or soil-based models and process-based models have similar predictive power (Sala et al. 1988 or Burke et al. 1991 vs. Parton et al. 1987.1994). Intercepted solar radiation has also been suggested as a major control over NPP, although Field (1991) pointed out the circularity inherent in observed correlations between intercepted radiation and plant growth.

The climatic versus nutrient limitation views inherent in modern ecology (discussed in Schulze et al. 1989 and Schimel et al. 1991) have important implications for predictions of future environmental changes, and for the application of models in land management. Keeling 
et al. (1995) argued that on the decadal time scale, the observational record suggests that warming causes increased $\mathrm{CO}_{2}$ uptake by the biosphere, but with a two year lag. This phenomenon, if true, is inconsistent with direct biophysical effects, which are nearly instantaneous, but is consistent with responses to a warming-induced release of nutrients and subsequent increases in NPP, as is suggested by models of long-term effects (VEMAP 1995).

In a recent study, response of modeled carbon storage to increasing $\mathrm{CO}_{2}$ was reduced by increasing nutrient limitation (VEMAP 1995). Other work suggests temperature responses will decrease as well (Schimel et al. 1994). The temperature response occurs because warming increases microbial respiration, thereby causing reductions in soil carbon; but warming releases nutrients, which, in forests, can lead to increases in carbon storage in wood that are larger than the carbon losses from soils (VEMAP 1995). Because of this, the short-term effect of warming (carbon losses from soils) may be opposite in sign to the long-term effect (carbon gains in wood). However, if nutrient cycling equilibrates rapidly with climate and $\mathrm{CO}_{2}$ determinants of carbon storage (on annual to decadal time scales, e.g., through nitrogen fixation; Gifford 1994. Schimel 1995a, Schimel et al. 1995), then nutrient feedbacks may be neglected in the big picture, and attention focused on $\mathrm{CO}_{2}$ and biophysical constraints. If nutrient cycling is uncoupled from climate and $\mathrm{CO}_{2}$ changes (e.g., controlled more by long-term pedogenic and geomorphic processes; Cole and Heil 1981, Schimel et al. 1985), then modeling nutrient cycles is essential to simulating future changes to ecosystems.

\subsubsection{Model description and methods}

Over the past decade, the Century terrestrial ecosystem model has been developed, which now includes parameterization of the major pathways for carbon and nitrogen exchange, including atmospheric and biological $\mathrm{N}$ inputs, and gaseous, biomass combustion-related, and 
hydrological N losses (Parton et al. 1987,1988,1994. Schimel et al. 1990,1991,1994. Ojima et al. 1994). Examination of the controls over carbon and nitrogen dynamics in the Century ecosystem model suggests a mechanism through which biophysical (water and temperature) and biogeochemical controls may become correlated. The distinguishing features of Century are, first, the explicit partitioning of living biomass and dead organic matter into compartments defined by differing turnover times, and second, the high degree of integration between biophysical and biogeochemical processes. For the living components, the model explicitly considers leaves, fine roots, coarse roots, branches, and stems, and for dead organic matter the model is based on isotopic and other evidence for multiple turnover times in detritus and soil organic matter (Trumbore 1993, Parton et al. 1994,1995). The structure of the litter/soil carbon submodel is shown in Fig. 4.1. Full descriptions of Century are found in Parton et al. (1987,1993,1995): below we describe features of the model and its global implementation that are new and/or impact the results described in this paper.

The gridded version of the model requires three types of information, including inputs that are grid cell-specific and time varying (largely those related to the atmosphere), inputs that are grid cell-specific and fixed (mostly soil properties), and variables that describe physiological attributes of vegetation types that apply to all grid cells within a vegetation type. The model is integrated using monthly maximum and minimum temperatures and precipitation. Climate data used in this study are as described in Cramer et al. (1995).

The algorithm for NPP in Century is key to understanding the results presented in this section. In Century, a "potential NPP" $\left(N P P_{p}\right)$ is computed using the following equation:

$$
N P P_{p}=N P P_{\max } \cdot T \cdot M \cdot S,
$$




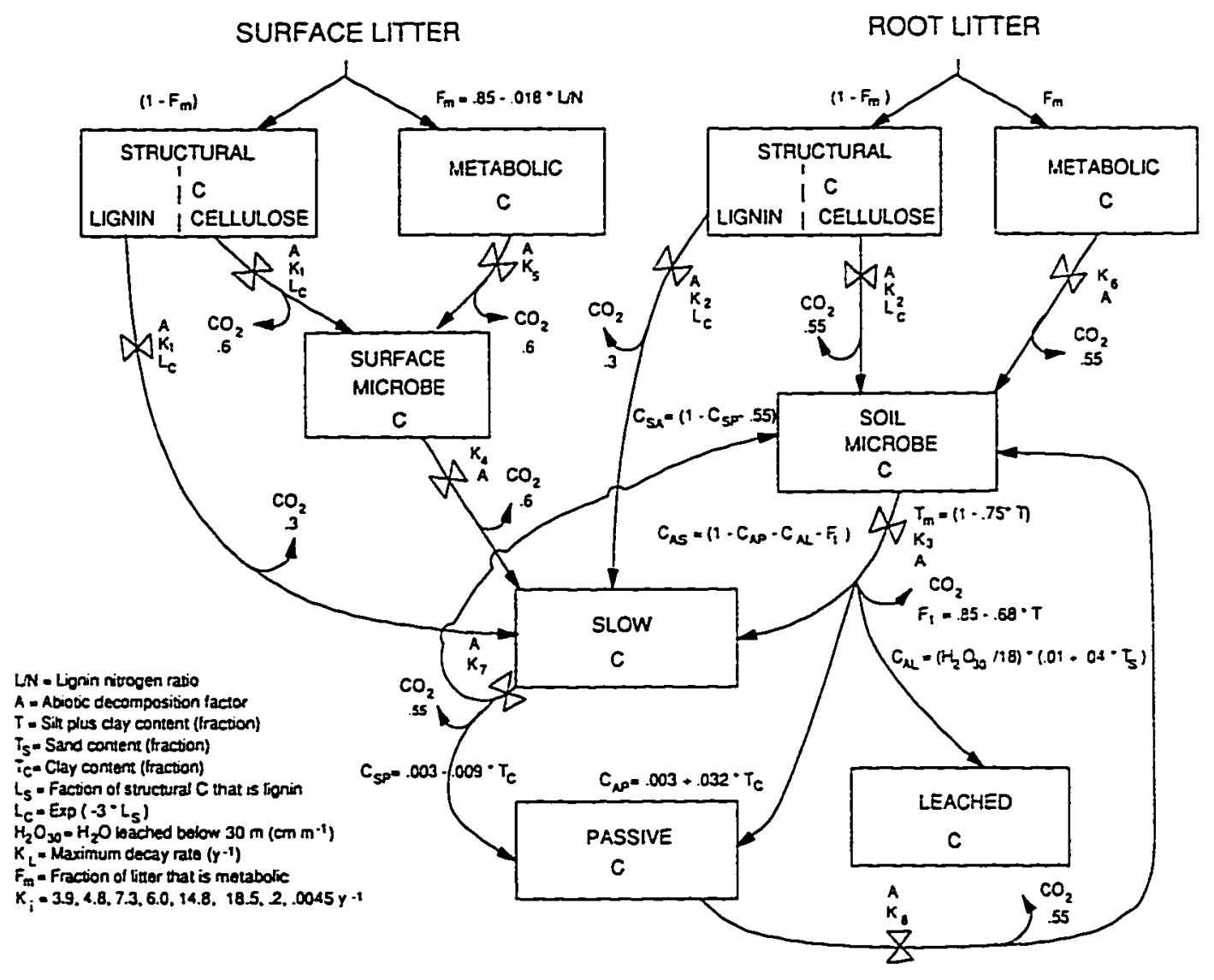

Figure 4.1 Structure and main parameters of the carbon flow submodel of Century. The locus of controls are indicated within the figure, and the controlling parameters are indicated adjacent to the figure. Nitrogen fluxes are essentially in parallel with the $C$ fluxes (Parton et al. 1993). 
where $N P P_{\max }$ is a biophysically defined maximum NPP, $T$ is the effect of temperature on NPP, $M$ the effect of soil water, and $S$ the effect of self-shading. The functional forms for $T, M$ and $S$ are given in Parton et al. (1993). This climatically-limited NPP is then constrained by nutrient availability. The nutrient-limited NPP equation estimates the fraction of $N P P_{p}$ that can be achieved while maintaining appropriate tissue $\mathrm{C}: \mathrm{N}$ stoichiometry:

$$
N P P_{n}=\sum_{i=1}^{m}\left(N_{\text {avail }} \cdot w_{\text {root }}+N_{\text {fix }}\right) \cdot F_{i} \cdot C N_{i},
$$

where $N P P_{n}$ is the nutrient-limited NPP, $N_{\text {avail }}$ is the available mineral $\mathrm{N}$ in soil solution $\left(N_{\text {sol }}\right)$ plus $\mathbf{N}$ in plant storage pools (plant storage results from $\mathrm{N}$ that is resorbed from senescent foliage and is available for new growth in the spring, or following drought in perennial vegetation). In turn, $N_{\text {sol }}$ is the sum of fixation plus atmospheric deposition. The root impact factor $w_{\text {root }}$ weights plant nutrient uptake based on root biomass (if root biomass is zero, $w_{\text {root }}$ is zero; at an upper threshold of root biomass, $w_{\text {root }}$ is one). $F_{i}$ and $C N_{i}$ are the fraction of total potential plant $\mathrm{N}$ uptake $\left(N_{\text {avail }} \cdot w_{\text {root }}+N_{\text {fik }}\right)$ allocated to plant part $i$, and the $\mathrm{C}: \mathrm{N}$ ratio of plant part $i$, respectively. These are indexed over the $m$ plant parts (roots, leaves, fine and coarse wood). The $\mathrm{C}: \mathrm{N}$ ratios of plant parts float between tissue type-specific upper and lower critical levels $\left(C N_{i, m i n}\right.$ and $\left.C N_{i, m a x}\right)$ and are scaled by function of supply divided by demand $D$ :

$$
C N_{i}=f\left\{\left(N_{\text {avail }} \cdot w_{\text {root }}+N_{f i x}\right) / D\right\}
$$

where $D$ is equal to $\Sigma_{i}\left(F_{i} \cdot N P P_{p} / C N_{i, m i n}\right)$. The scalar function $f$ is defined such that $C N_{i}$ equals $C N_{i, \max }$ when supply equals demand, and approaches $C N_{i, \min }$ when supply is limiting. That is, $\mathrm{C}: \mathrm{N}$ ratios are scaled by the ratio of $\mathrm{N}$ available for plant uptake divided by the amount of $\mathrm{N}$ 
that a plant would use if it could get all the nitrogen needed to grow at the climate-limited rate. Thus the higher $N P P_{p}$ is relative to $N P P_{n}$, the wider the plant $\mathrm{C}: \mathrm{N}$ ratios. Then, in effect, actual NPP $\left(N P P_{a}\right)$ is defined by:

$$
N P P_{a}=\min \left(N P P_{n}, N P P_{p}\right)
$$

Although $N P P_{n}$ is nearly always less than $N P P_{p}$, if $N P P_{p}$ goes up. $N P P_{a}$ will generally follow, due to Equation 4.4, which allows $\mathrm{C}: \mathrm{N}$ ratios to float based on the $\mathrm{N}$ supply/demand equation (unless the lower critical $\mathrm{C}: \mathrm{N}$ ratio threshold is reached). Experimental evidence supports floating C:N ratios between years (Schimel et al. 1991). If $\mathrm{N}$ availability goes up (e.g., if N mineralization increases due to warmer or wetter soils; Schimel et al. 1994), NPP will increase as $N P P_{n}$ more nearly approaches $N P P_{p}$. Conditions that modify $N P P_{p}$ (changes to temperature or precipitation) will also affect soil processes (and hence $N_{\text {avail }}$ ) that are also influenced by $T$ and $M$ (see Schimel et al. 1994 and Parton et al. 1995).

The model requires estimates of nitrogen fluxes into the system. As discussed below, budgetary nitrogen fluxes are critical to the model. Nitrogen enters ecosystems via several mechanisms including wet and dry deposition. We assume, based on extensive data analysis, that deposition is correlated with precipitation, and we use this correlation to produce a general field of $\mathrm{N}$ deposition mimicking observations (Parton et al. 1987, Schimel et al. 1990). This relationship does not supply sufficient nitrogen in tropical regions to support observed NPP; assuming that this difference is due to greater biological fixation in the tropics, a second equation relates $\mathrm{N}$ input due to fixation to actual evapotranspiration $\left(E T_{a}\right)$ :

$$
N_{f i x}=0.008 \cdot\left(E T_{a}-40\right)
$$


If $N_{f i x}$ is less than zero $\left(E T_{a}<40 \mathrm{~cm} \mathrm{yr}^{-1}\right)$, then it is set to zero. This parameterization has no mechanistic basis but captures phenomenologically the fact that most observations of high rates of biological nitrogen fixation are from humid settings with high evapotranspiration, and that these systems (tropical and temperate rainforests) have large $\mathrm{N}$ stocks and high losses (requiring high inputs) of $\mathrm{N}$ (e.g., Matson and Vitousek 1987). In Century, this provides a constant flux, tuned to provide sufficient $\mathbf{N}$ to sustain observed NPP. In reality, biological nitrogen fluxes may occur at certain successional or climatic stages, rather than as a constant background process. The requirement to use this empirical and tuned parameterization reflects our ignorance of the biogeography and biogeochemistry of nitrogen fixation in natural ecosystems over long time scales (Eisele et al. 1989, Vitousek and Howarth 1991, Schimel 1995a).

Soil properties required by Century include soil texture and depth. Texture is defined by the continuous distribution of soil in terms of size classes (percentage of sand, silt, and clay). These may be derived via a look-up table from maps in which texture is defined categorically; in this case the mid-point percentages of size classes are used for each category. For the simulations presented in this paper, soil textures were prescribed according to Zobler's (1986) analysis of the FAO Soil Map of the World (see also Schimel et al. 1994).

Century also requires some vegetation type-specific parameterizations (VEMAP 1995, Schimel et al. In press b). These include carbon-to-nutrient ratios by tissue type and nitrogen allocation between tissue types for each ecosystem type. Lacking a comprehensive theory of allocation, fundamental allocation relationships (fraction of NPP allocated to wood vs. foliage vs. roots) are defined as a function of ecosystem type, and as a function of plant size via allometric equations, designed to reproduce observations at calibration sites. This approach is 
based on a large data base, reproduces observed allocation patterns at calibration and validation sites, and allows some changes in allocation proportions as trees or large shrubs become larger. It does not reproduce changes in allocation resulting from changes in resource availability, which have been observed and which may be significant (Ingestadt and Lund 1986, Running and Hunt 1993). We adopted the more conservative approach of using parametric allocation equations because of the lack of general and proven theory for a resource-based approach (Running and Hunt 1993), and the lack of parameters for a resource-based approach for many biomes.

Three model experiments are reported in this paper. First, we carried out a global steady state simulation, in which Century was integrated using global climatology, soils, and vegetation descriptions as described above. That simulation was carried out according to the protocols of the Potsdam Intercomparison of the IGBP Global Analysis, Interpretation, and Modeling (GAIM) Task Force (see Cramer et al. 1995).

Second, we carried out a temperature anomaly experiment on a globally-distributed subset of grid cells in which we modified the temperatures based on the deviations from longterm averages that occurred over the period 1981-1994. The deviations (anomalies) were based on satellite measurements using the Microwave Sounding Unit (MSU) aboard NOAA's polar orbiting spacecraft (Spencer et al. 1990). The long-term monthly means were subtracted from each monthly value to create an anomaly time series with zero mean. The deviations were added to or subtracted from the climatological values. The MSU temperatures are for the lower troposphere, and so are not precisely the same as surface temperatures, although the anomalies are usually highly correlated. Monthly precipitation was held constant at climatological values in this simulation. Because surface observations in high latitudes are very sparse, the satellitebased approach has significant advantages. The satellite temperatures are global measurements 
and avoid the problems of interpolation from station data. As will be seen, temperature anomalies in the Northern Hemisphere high latitudes are quite significant.

Third, we carried out a temperature perturbation experiment. At a subset of sites, we integrated the model to quasi-steady state and then perturbed the model by increasing monthly temperatures by three standard deviations over a period of either 1 or 5 years, and then retumed temperatures to their long-term mean. The model integration was then continued for three additional decades. This experiment was carried out to determine the time scales of response to physical forcing, and to aid in the interpretation of the temperature anomaly experiment described above.

\subsubsection{Results and discussion}

Nutrient and water limitation equilibration at steady state

The water-carbon-nutrient relationships in Century emerge from its basic logic (see Equations 4.2-4.5, above); wherein the modeled fluxes of nitrogen through ecosystems are strongly influenced by the hydrological cycle. Inputs of $\mathrm{N}$ are either directly linked to precipitation (wet deposition) or assumed to be correlated with annual evapotranspiration (nitrogen fixation). Losses of nitrogen are similarly controlled by water. Leaching losses of $\mathrm{NO}_{3}{ }^{-}$and dissolved organic $\mathrm{N}$ (DON) are directly controlled by the product of water flux and $\mathrm{NO}_{3}{ }^{-} / \mathrm{DON}$ concentrations (Parton et al. 1994). Losses of $\mathrm{N}$ trace gases are linked to mineralization of $\mathrm{NH}_{4}{ }^{+}$and $\mathrm{NO}_{3}{ }^{-}$from organic matter. The rate of loss is controlled by an abiotic decomposition factor that increases as temperature, rainfall, and soil moisture increase (Parton et al. 1994, Holland et al. 1995). Losses of gaseous $\mathrm{N}$ from inorganic $\mathrm{N}$ (proportional as well as absolute) also increase with increasing soil moisture (Parton et al. 1988). Thus the flux of $\mathrm{N}$ through ecosystems will in general increase as precipitation increases. Critical 
controls over soil moisture and water flux through the soil include the atmospheric demand for water in evapotranspiration, and the water holding capacity of soils. Thus, although we argue that the hydrological cycle is a major control over $\mathbf{N}$ cycling, implicitly both energy (as a driving force for ET) and soil hydrological properties substantially modify the operation of the hydrological cycle from grid cell-to-grid cell.

Potential primary production increases as evapotranspiration increases in Century because the model includes an equation that constrains primary production by linking the sum of rainfall and previously stored water (divided by potential evapotranspiration) to production (Equation 4.1) (Parton et al. 1994). This equation integrates the precipitation, energy, and soil hydrological constraints over the evapotranspiration flux. Additionally, primary production requires nitrogen to form organic matter meeting critical $\mathrm{C}: \mathrm{N}$ ratios for wood, foliage, and roots (Equations 4.2 and 4.3).

$\mathrm{N}$ is derived from precipitation and $\mathrm{N}$ fixation on centennial time scales, but on an annual time scale most plant-available $\mathrm{N}$ is derived from nitrogen mineralization, which arises from the turnover of dead organic matter (decomposition). To illustrate the time scales of the $\mathrm{N}$ cycle, consider that nitrogen inputs range from $10-30 \%$ of $\mathrm{N}$ mineralization. $\mathrm{N}$ mineralization, in turn, averages $<5 \%$ of soil organic $N$. Nitrogen mineralization is derived from the turnover of compartments with turnover times from $1-8$ years $(\sim 80 \%)$ and $30-90$ years $(\sim 20 \%)$ (Schimel et al. 1994). Typically, nitrogen losses very nearly equal inputs. Nitrogen losses due to trace gas emission and leaching range from 5 to $40 \%$ of inputs. Episodic losses due to fire and tree mortality, averaged over the modeled disturbance cycle, bring losses to near-equality with inputs. Because $\mathrm{N}$ inputs range from $0.4-1.5 \mathrm{~g} \mathrm{~m}^{-2} \mathrm{yr}^{-1}$, and soil nitrogen levels typically exceed $500 \mathrm{~g} \mathrm{~m}^{-2}$, nitrogen storage can only increase at a fraction of a percent per year even if losses are zero. 
The structure of the model results in cyclic coupling of production and decomposition during the approach to steady state (Fig. 4.2). Forest NPP in Century approaches a constant value as the nitrogen budget approaches balance (inputs equal to outputs). However, when NPP stabilizes, net ecosystem production (NEP) may still be small and positive in forests, because of the continuing accumulation of carbon in wood with minimal requirements for additional nitrogen, which are met by small changes in storage or nitrogen use efficiency. Thus for forests, Century simulates a quasi-steady state.

As water flux increases, $\mathrm{N}$ flux increases, and likewise, the potential for carbon fixation increases. As carbon fixation increases, the amount of the $\mathrm{N}$ flux that can be captured in organic matter increases. As more nitrogen is captured in organic matter, its subsequent turnover also contributes to plant-available $\mathrm{N}$, allowing more plant productivity. These processes contribute to accumulation of living and nonliving organic matter reflecting the water budget. Figure 4.3 shows the global distribution of modeled NPP, ET, and nitrogen mineralization. Strong correlations between ET, nitrogen availability, and net primary productivity appear in global steady state simulations (Fig. 4.4a-c). The relationships amongst ET, N mineralization, and NPP are modulated on a grid cell-to-grid cell basis by other factors that influence turnover times, such as temperature, ecosystem type-specific factors that control resource use efficiencies (effectively the carbon-to-nutrient stoichiometry of plants and microorganisms), and water use efficiency (organic matter produced per unit water transpired). ET itself is also modified by physical soil and energy budget variables that influence the partitioning of precipitation to transpiration, evaporation, and runoff, with consequent effects on water use efficiency.

Whereas large-scale patterns in NPP-resource relationships arise from system-level interactions of the biogeochemical and hydrological cycles, substantial variation is induced by 


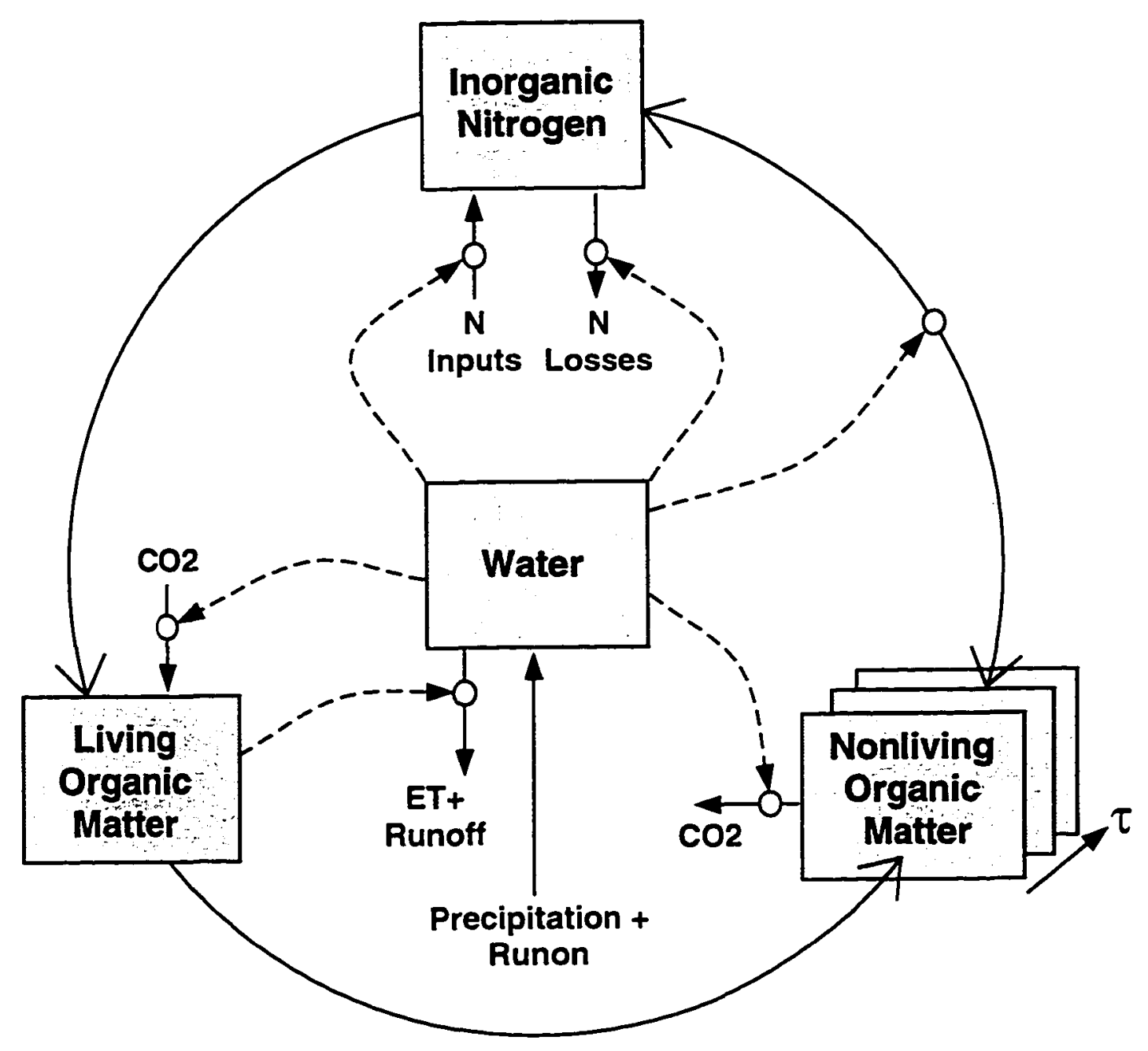

Figure 4.2 The cyclic coupling of carbon, water flux, and nitrogen cycle processes, idealized from Century. Critical points include the control of the nitrogen budget by the hydrological cycle, the feedbacks between carbon and water dynamics, and the effect of carbon flux on the capture of nitrogen into organic forms. 

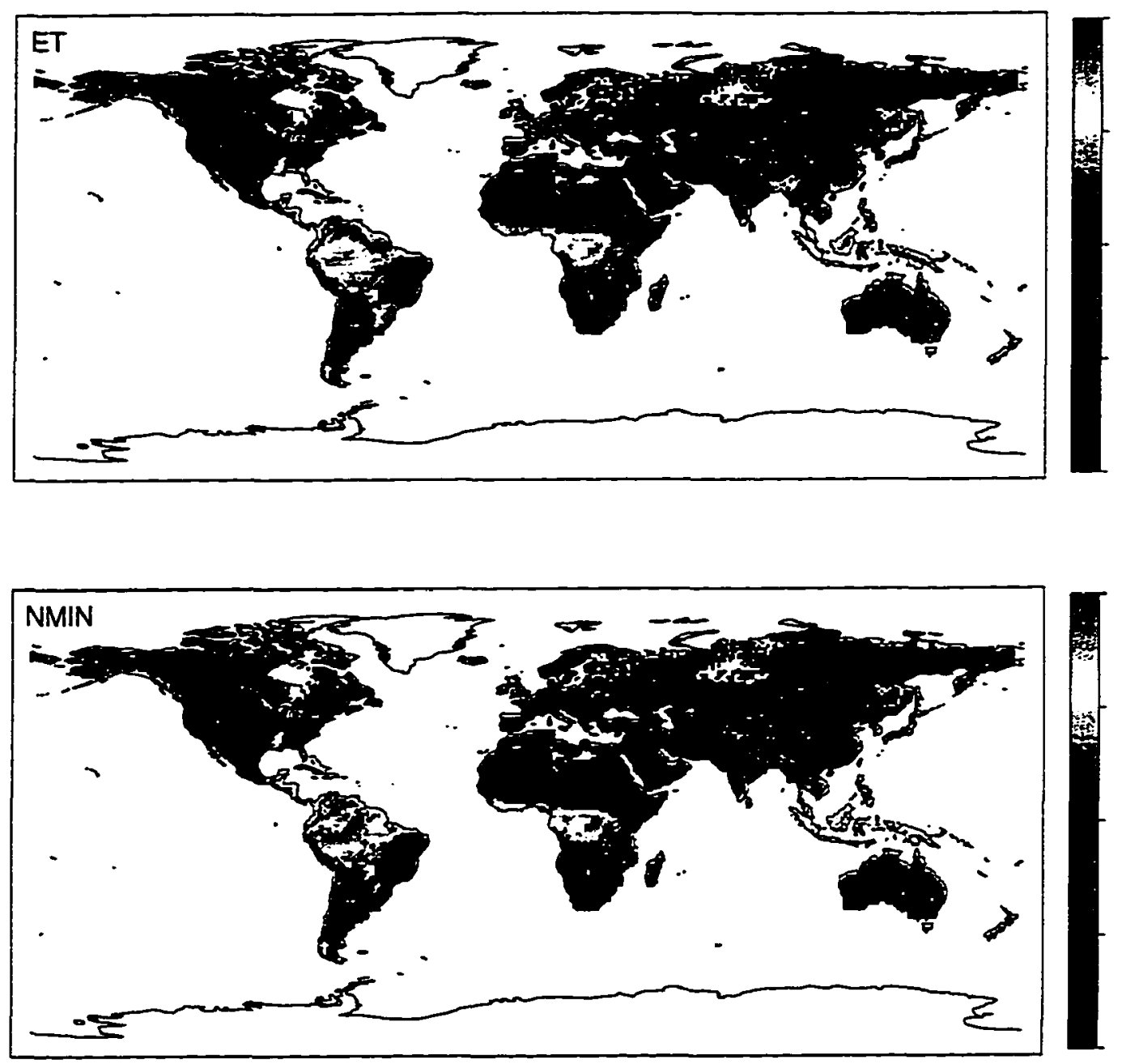

to

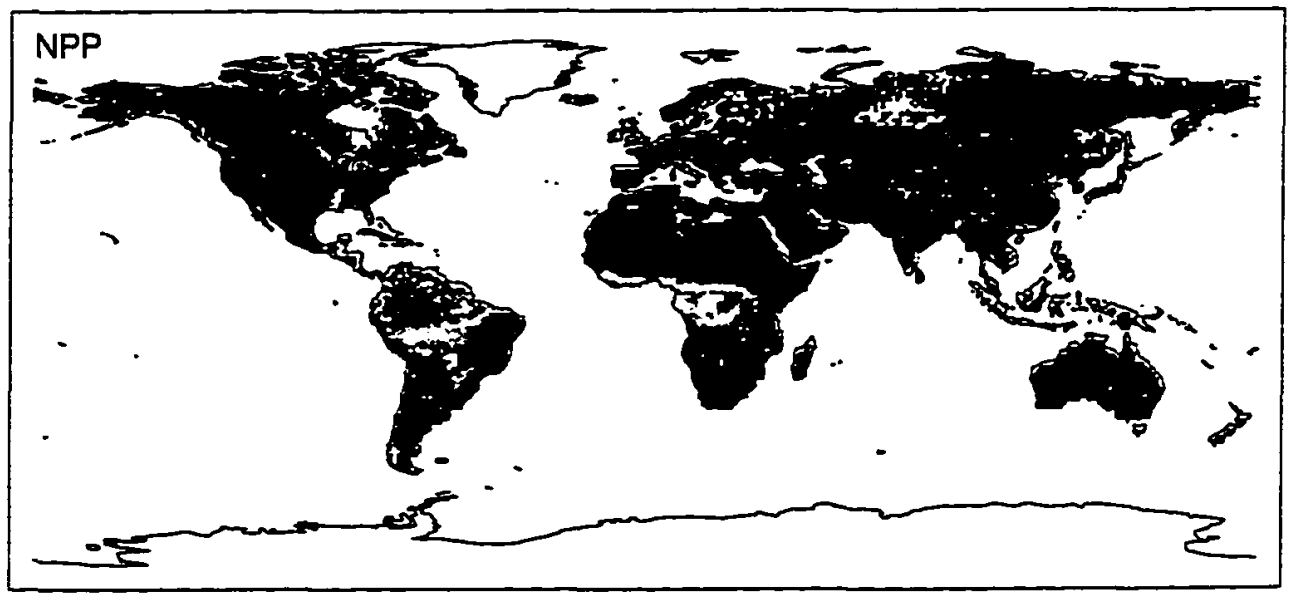

Figure 4.3 (a) Mapped annual evapotranspiration from a global steady state integration of Century. (b) Mapped nitrogen mineralization (the net production of $\mathrm{NO}_{3}^{-}$plus $\mathrm{NH}_{4}^{+}$from organic matter). (c) Mapped net primary production. 

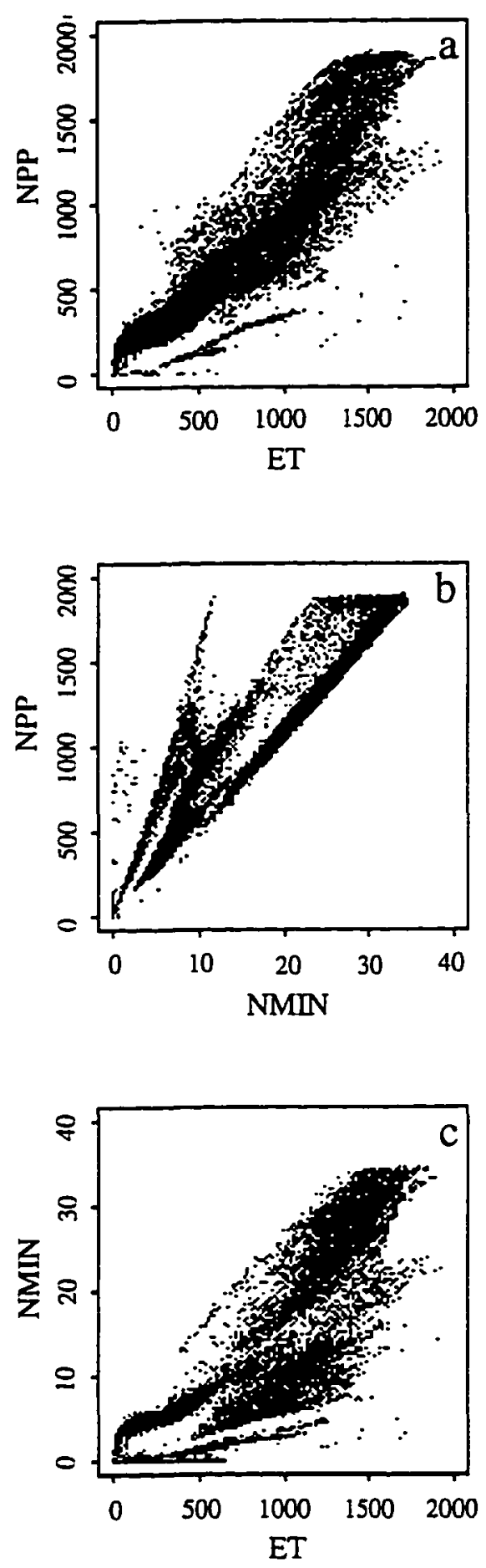

Figure 4.4 (a) The relationship between NPP and ET, from Fig. $4.3 \mathrm{a}, \mathrm{b}\left(R^{2}=0.86\right)$. (b) The relationship between $\mathrm{N}$ mineralization and NPP, from Fig. $4.3 \mathrm{~b}, \mathrm{c}\left(R^{2}=0.92\right)$. (c) The relationship between $\mathrm{N}$ mineralization and $\mathrm{ET}$, from Fig. 4.3a, $\mathrm{c}\left(R^{2}=0.75\right)$. Green $=$ forest, black $=$ savanna, yellow $=$ grassland . 
species- and growth form-specific traits. The correlation of variables in Fig. 4.4a-c indicates the extent to which NPP and nitrogen cycling are controlled by system-level dynamics: the scatter and variability of slopes indicates roughly the extent to which system-specific ecological traits influence NPP and nitrogen cycling.

The relationships in Fig. 4.4 appear as trends, with different slopes and/or intercepts for different ecosystem types, rather than as unitary relationships. Although each of the biome types used in parameterizing global Century have specific resource use efficiencies, it is apparent that some large scale patterns exists between forests, grasslands, and mixed ecosystem types, such as savannas. Systems with woody components have higher $\mathrm{N}$ use efficiencies (NPP vs. NMIN) (e.g., Fig. 4.4b), as do C4 compared to C3 photosynthetic pathway grasslands. Savannas that have both woody and herbaceous components show $\mathrm{N}$ mineralization-NPP relationships similar to but slightly higher than grasslands. Within savannas, a similar bifurcation is observed, reflecting the differentiation between savannas with $\mathrm{C} 3$ vs. $\mathrm{C} 4$ grass understories. A C3-C4 effect is also observed in the $\mathrm{N}$ mineralization-ET relationship. In this case, differences in nitrogen use efficiency between $\mathrm{C} 3$ and $\mathrm{C} 4$ grasses result in changes to detrital C: $\mathrm{N}$ ratios, that in turn influence $\mathrm{N}$ mineralization.

Water use efficiency (NPP per unit ET) increases as the fraction of woody biomass increases (Fig. 4.4). This pattern reflects the use of ET, rather than transpiration in these Figs. The modeled ratio of evaporation to transpiration generally increases from forests to grasslands in Century, and produces part of this pattern. Also, although grasses (especially C4 grasses) have high photosynthetic water use efficiency, Century predicts that the fraction of photosynthate translated into NPP increases as the nitrogen demand of new tissue decreases. Thus systems dominated by woody tissue have higher effective water use efficiencies at the annual time scale than grasslands because of $\mathrm{N}$ constraints on the production of high- $\mathrm{N}$ foliar 
tissue in grasslands. This is amplified by higher absolute rates of $\mathrm{N}$ cycling in humid forest ecosystems compared to grasslands (Fig. 4.3b). The interaction of water and nitrogen use efficiencies in Century is consistent with the biogeochemical orientation of the model (VEMAP 1995, Schimel et al. In press b) and suggests that model comparisons with annual time scale observations of water and nitrogen use efficiencies between grasslands, savannas, and forests (as can be derived from flux experiments; Wofsy et al. 1993) are crucial for model testing.

There are limited observations to support the hypothesis of tripartite correlation amongst N, water flux, and NPP, but Zak et al. (1994) report correlations amongst an index of nitrogen availability, aboveground NPP, and ET. The model results are also consistent with observations of large-scale correlations of NPP with direct or derived climate variables, and also with experimental evidence of nutrient limitation. This result is consistent with the argument of Pastor and Post (1993) that the Rosenzweig (1968) relationship of NPP to actual evapotranspiration (AET) was a "steady state consequence of the interactions between species and soil nitrogen and light availabilities as constrained by temperature and soil water deficits," and not a "direct relationship between actual evapotranspiration and productivity." The modulation of the water-carbon-nitrogen system by species- and/or growth form-specific traits implies that large-scale dynamics are influenced by population dynamics on time scales longer than the life-spans of individual plants (years to centuries).

Because of the sensitivity of Century to resource use efficiency and allocation patterns, a knowledge of the spatial and temporal distributions of physiological traits (either for species or functional plant types) is critical for modeling biogeochemistry. Knowledge of how these traits might change with adaptation, evolution, or migration is critical in the long run for predictive or retrospective analyses on time scales commensurate with ecosystem type change (Bolker et al. 1995). 


\section{Temperature anomalies}

Temperature variability (Fig. 4.5) causes biogeochemical responses in the Century model. In the temperature anomaly experiment, highly variable correlations over time between NPP, respiration, and NEP with temperature were observed (Fig. 4.6). The correlations with temperature tended to be higher as precipitation increased. In systems where water is strongly limiting to NPP, interannual variations in temperature have comparatively little effect. Conversely, we would expect such systems to be very responsive to interannual variations in precipitation (Parton et al. 1995). The lack of response to temperature is surprising; temperature is a direct driver of biogeochemical processes in Century, and one would expect a fairly direct correlation between temperature anomalies and resulting biogeochemical anomalies. In fact, the correlations are not always high (only a small number of the correlations in Fig. 4.6 are significantly different from zero at a $95 \%$ level), and the proportionality between changing temperature and changing biogeochemical response was highly variable. This occurs because of lagged effects of temperature via water budget and biogeochemical mechanisms, explored in the next section (on temperature perturbations and time scales).

One of the dramatic carbon cycle phenomena during the 1990s was the reduction in the growth rate of $\mathrm{CO}_{2}$ in 1992 , following the eruption of $\mathrm{Mt}$. Pinatubo in the Philippines and the consequent cooling due to stratospheric aerosols (Hansen et al. 1992, Ciais et al. 1995b, Francey et al. 1995, Keeling et al. 1995, Schimel 1995b). The effect of the Pinatubo eruption was a reduction in the growth rate of $\mathrm{CO}_{2}$ corresponding to an anomalous additional $\mathrm{CO}_{2}$ uptake of about $2 \mathrm{Pg} \mathrm{C}$, and isotopic evidence suggested a terrestrial rather than marine sink (Keeling et al. 1995, Ciais et al. 1995b). The MSU temperatures capture the Pinatubo cooling clearly (Fig. 4.5) and show it to have significant spatial structure, concentrated in the Northern Hemisphere. Cooling during the boreal growing season of up to $3^{\circ} \mathrm{C}$ is evident (Fig. $4.5 \mathrm{~b}$ ). 

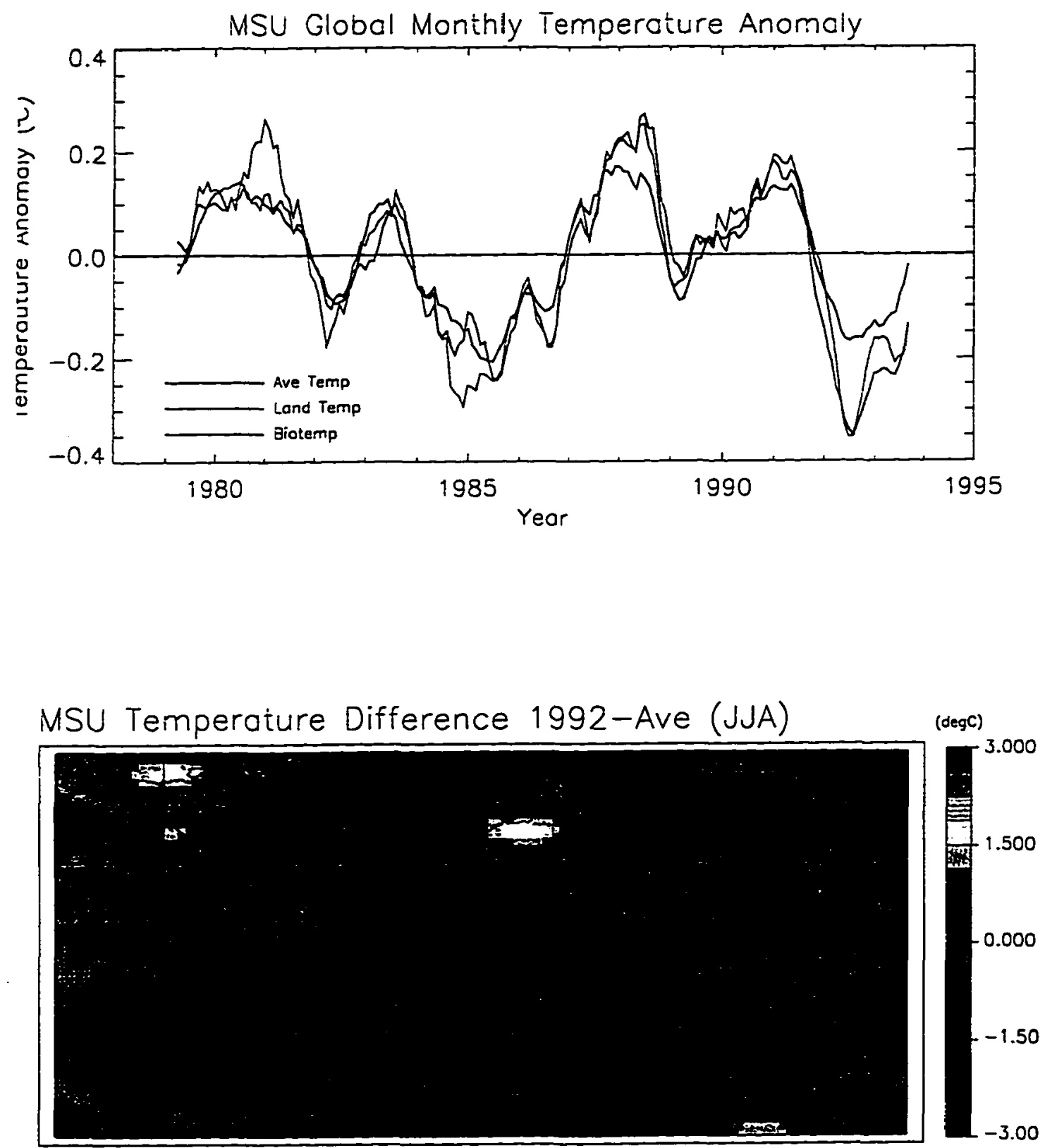

Figure 4.5 (a) Temperature anomalies from the MSU, showing the global average, the average over land, and the average over land during the Northern Hemisphere summer (using biotemperature). (b) The spatial distribution of the Boreal growing season temperature anomalies following Pinatubo ( $\mathrm{JJA}_{1992} \mathrm{JJA}_{\text {ave }}$ ). 


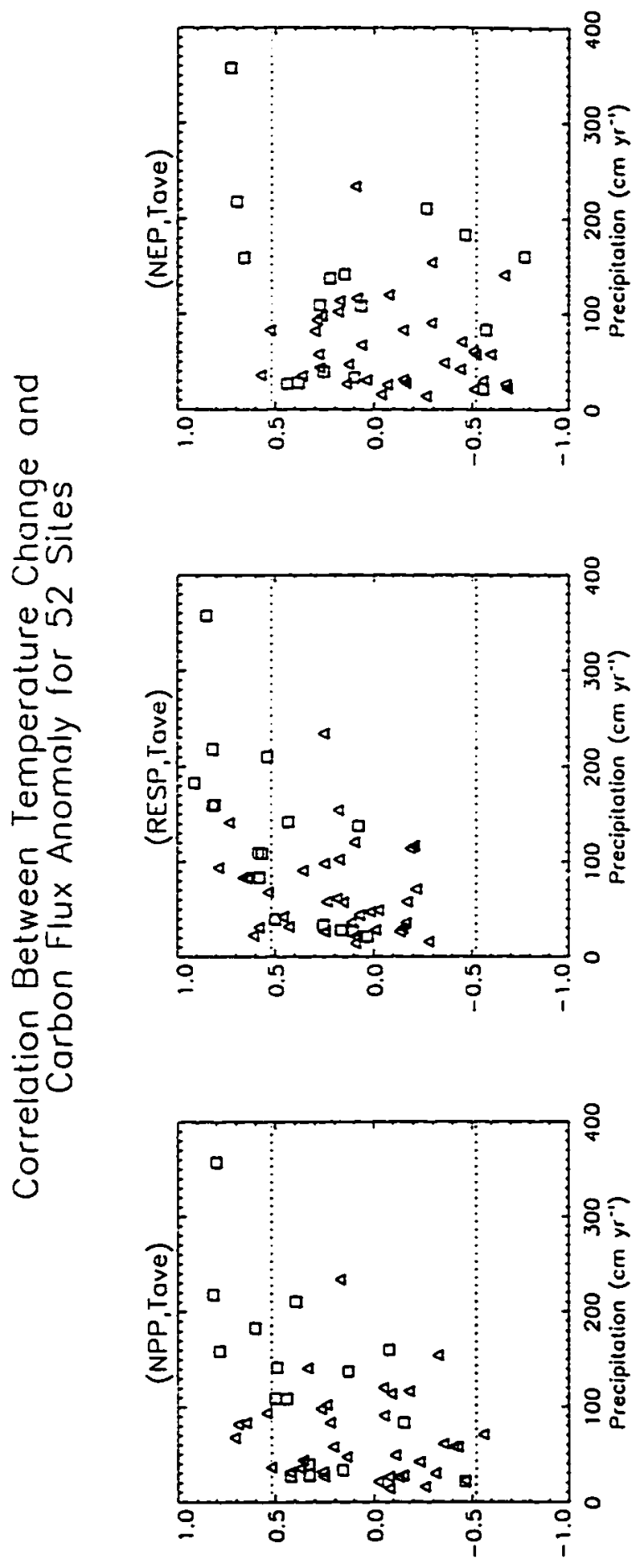

Figure 4.6 Correlations for 52 grid cells: (a) between temperature and NPP anomalies, plotted against precipitation; (b) between temperature and heterotrophic (microbial) respiration anomalies, plotted against precipitation; (c) between temperature and NEP anomalies, plotted against precipitation. Horizontal lines show $95 \%$ confidence intervals for $R$ significantly different from zero. Squares represent grassland sites and triangles represent forest sites. 
Despite the complexity of the temperature response of Century, the model predicts significant increases in per-unit-area NEP in mid-latitudes $\left(30-60^{\circ} \mathrm{N}\right)$. Whereas the model predicts release of $\mathrm{CO}_{2}$ from high latitude regions (negative NEP), there is comparatively little biologically active land area in that latitude band. If the predicted average NEP of $+30 \mathrm{~g} \mathrm{~m}^{-2}$ was representative of the $30-60^{\circ} \mathrm{N}$ region (Fig. 4.7), that could easily amount to $1-3 \mathrm{Pg} \mathrm{C}$ globally. Thus the Century model results are consistent with a Pinatubo-related anomalous increase in NEP (arising mostly from reduced respiration rather than increased NPP) of magnitude comparable to the additional sinks deduced from observations. If the modeled responses to the cooling are correct, an overshoot in atmospheric $\mathrm{CO}_{2}$ should occur as the short-lived carbon pools, whose size increased during the cooling, relax back to equilibrium with warmer temperatures. However. because of the low and variable direct correlations between temperature and ecosystem processes, we propose a more complex hypothesis for the observed and simulated Pinatubo effect.

\section{Temperature perturbations and time scales}

Although biophysical and nutrient limitations of NPP tend to equilibrate with each other (Fig. 4.4; Schimel et al. In press b), the equilibration will not be instantaneous. The response times of the different carbon-water-nitrogen components of ecosystems are not identical, and some are long. It requires years for soil water turnover, decomposition, and nitrogen cycling to return to steady state after a perturbation because of inherent lags in the system. We carried out a simple simulation experiment in which we increased air temperatures each month by three standard deviations for the duration of either 1 or 5 years to probe the lagged responses suggested by the temperature anomaly experiment. Figure $4.8 \mathrm{a}$ shows the response for a northern boreal forest. NPP increases during the year of the perturbation because of a longer 


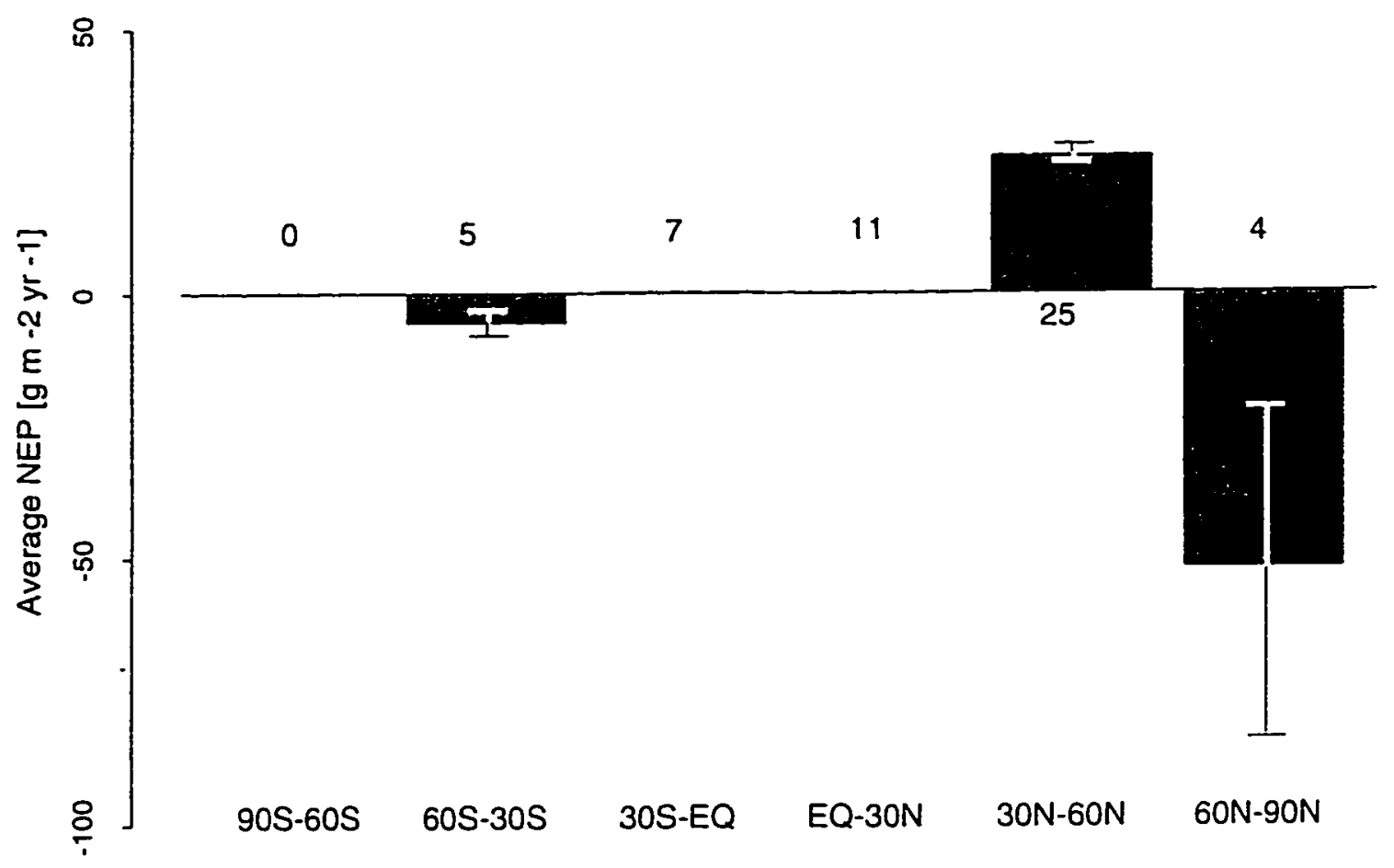

Figure 4.7 The effects of the Pinatubo temperature anomaly on net ecosystem production for 52 grid cells, averaged zonally. Error bars indicate mean \pm one standard error. 
a.
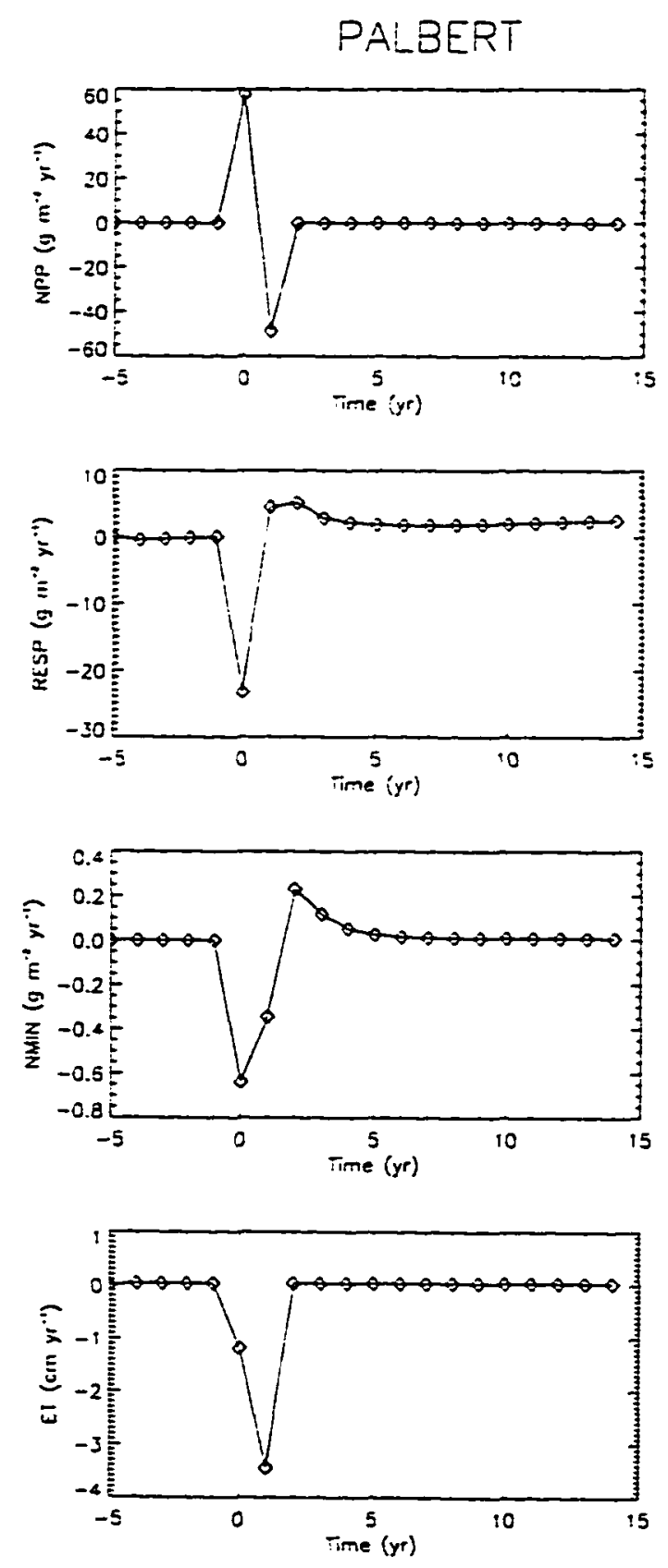

b.
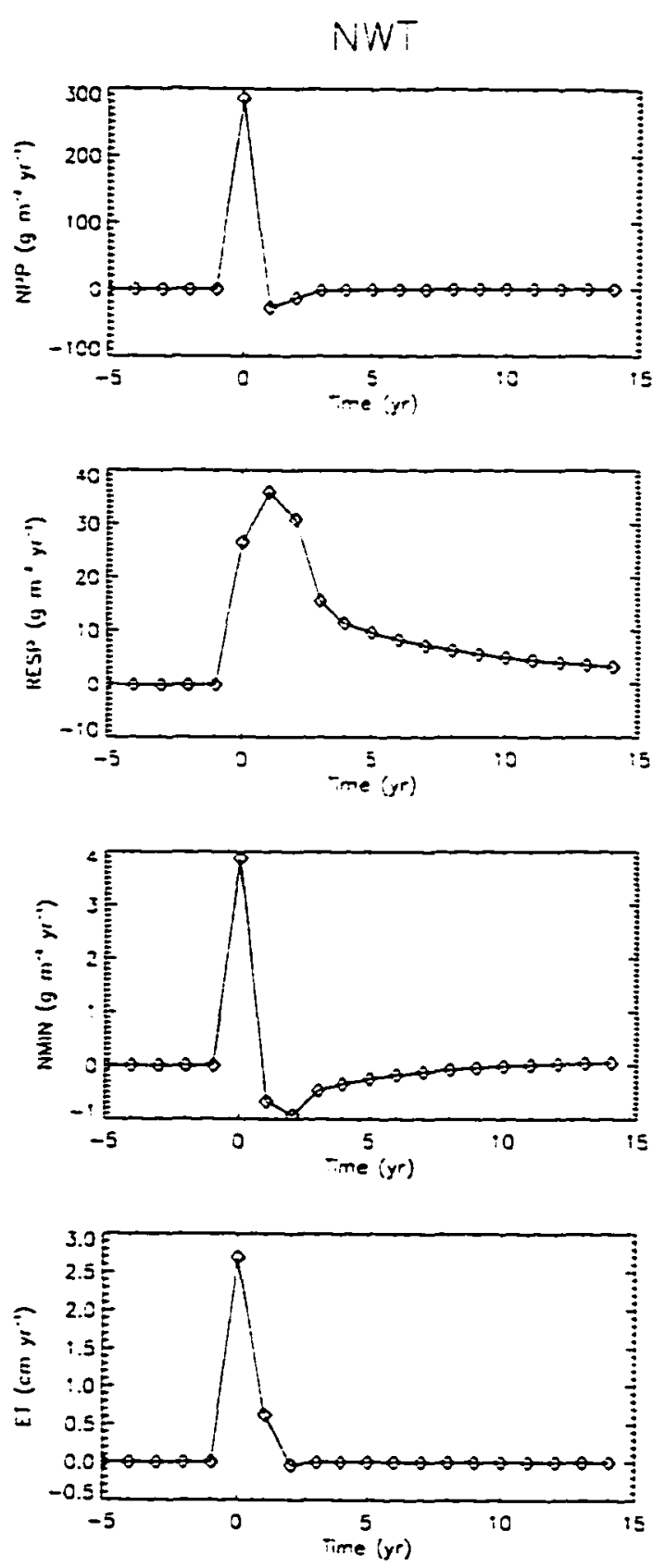

Figure 4.8 Results from a perturbation experiment in which the model, after integration to steady state, was perturbed with a 1-year warming by increasing each month's temperature by three standard deviations. (a) NPP, microbial respiration, nitrogen mineralization, and ET (top to bottom) for a northern boreal forest grid cell. (b) As in (a) but for an alpine tundra grid cell. Results are shown as deviations from steady state values. 
growing season and reduced temperature stress. Respiration (decomposition) decreases because warmer winter temperatures lead to reduced soil water (more evaporation from snow), reducing ET. $\mathrm{N}$ mineralization decreases, reducing NPP in the year following the perturbation. This occurs because of increased litterfall (from the enhanced NPP), and thus more microbial competition for $\mathrm{N}$, and because of dryer soils. Soil water is affected by the perturbation both directly and through the increased water demand associated with the initial increase in NPP. and returns to steady state after 3 years. The additional detritus resulting from the initial rise in NPP increases respiration beginning in year 2, and also leads to increases in both $N$ mineralization and respiration that persist for years.

Figure $4.8 \mathrm{~b}$ shows an alpine tundra site in which warming increases $\mathrm{N}$ mineralization. NPP, and ET. Increased NPP leads to increased respiration that persists for $>15$ years (because of slow decomposition in this cold, short growing season site), affecting net ecosystem production (NEP = NPP - heterotrophic respiration) for decades. Tropical sites (Fig. 4.9a) show similar lags, modulated by the water cycle. Respiration equilibrates rapidly because of high decomposition rates due to warm temperatures. When the temperature perturbation lasts for 5 years, complex behavior occurs as the system responds to the initial perturbation, equilibrates to the changed temperatures, and then responds again in a transient manner to the cessation of the temperature perturbation (Fig. 4.9b). The sites examined in Figs. 4.8? and 4.9? show NPP responses of opposite sign in the year of and following the perturbation; this is a frequent but not invariable behavior of the model. Other sites show large initial responses with monotonic returns to steady state, on time scales linked to component response times (Schimel et al. 1994).

The above responses were controlled by iterative feedbacks between the water, carbon, and nitrogen cycles, leading to complex responses on annual-to-decadal time scales. The 
a.
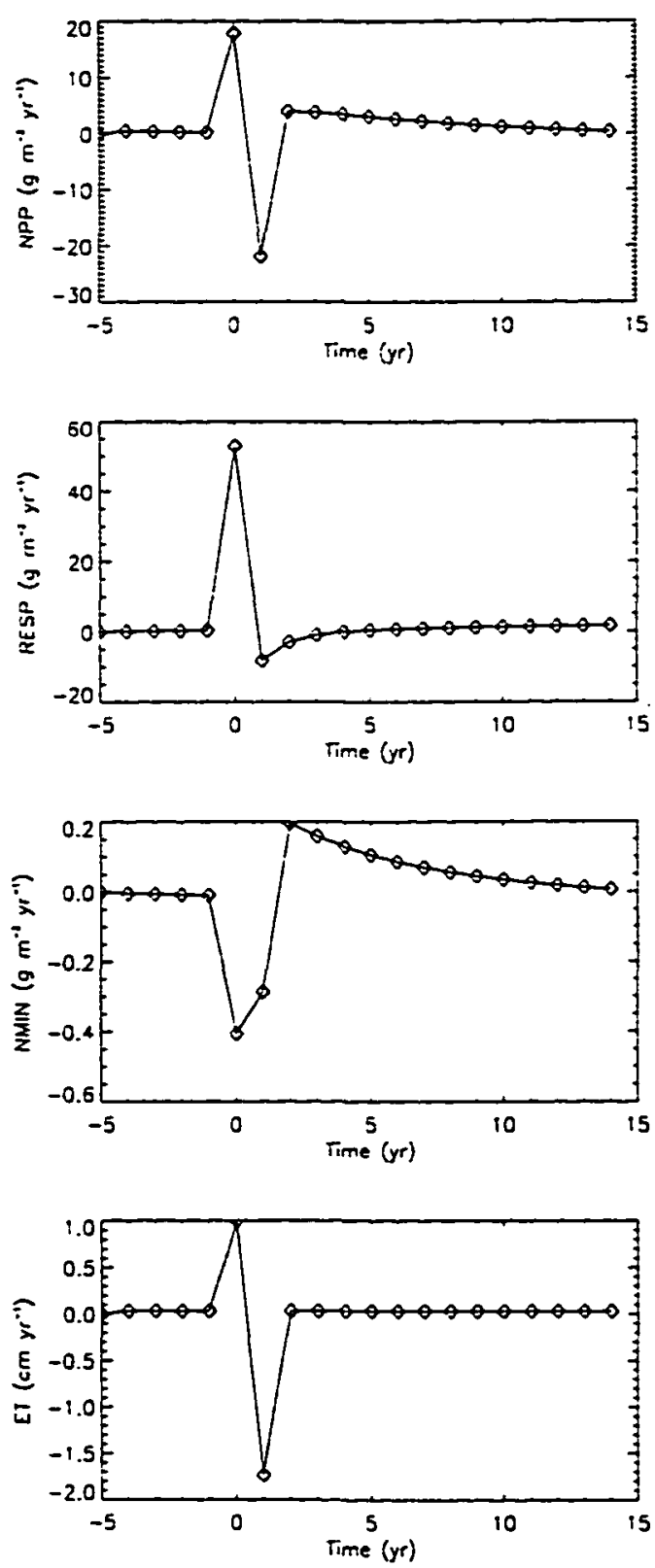

b.
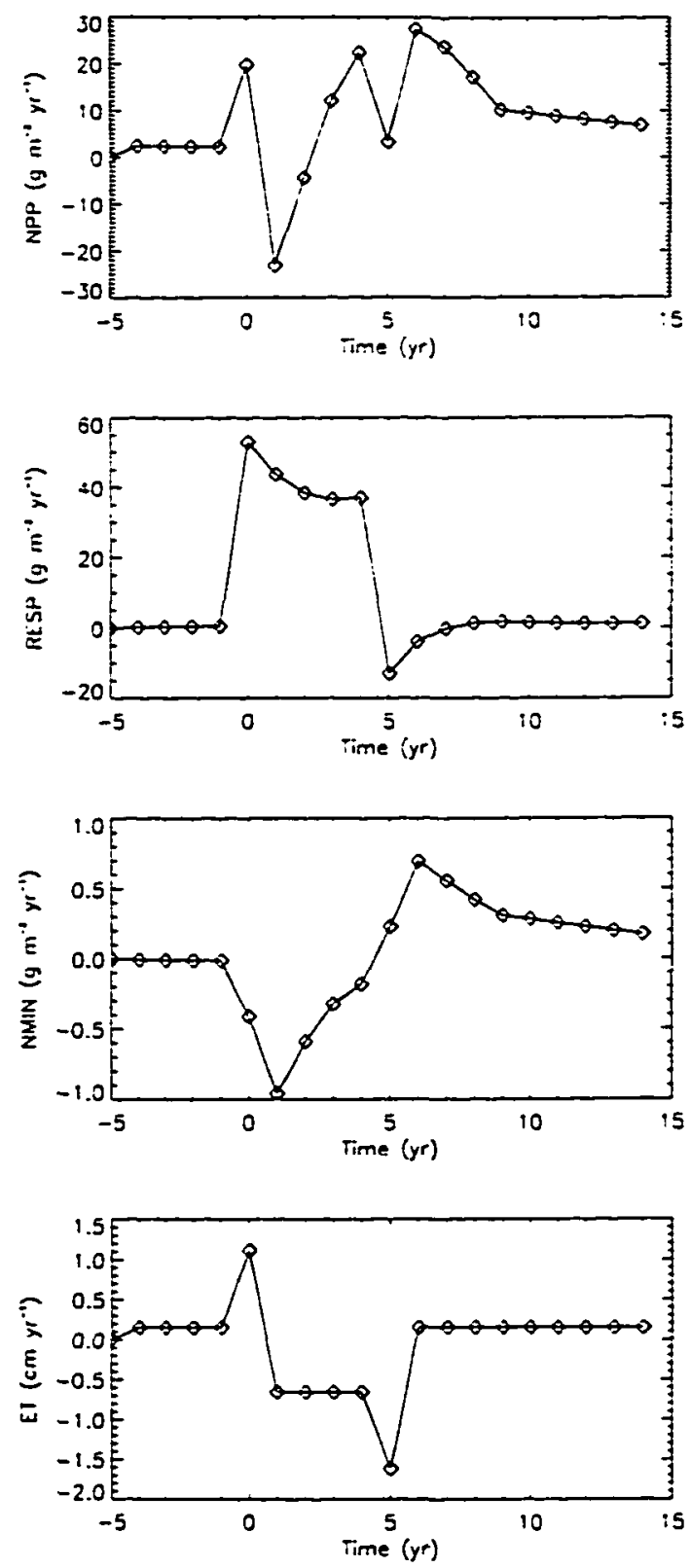

Figure 4.9 (a) As in Fig. 4.8 but for a tropical forest grid cell. (b) Using the same grid cell as (a) but showing results for NPP, respiration, nitrogen mineralization, and ET following a 5year temperature perturbation. 
responses were system-specific and were influenced by the initial steady state. In particular, systems in which precipitation often exceeds ET and in which stored soil moisture is significant, are sensitive to perturbations and may have multi-year responses to perturbations through the water cycle. The temperature perturbation experiment helps explain why the instantaneous correlations shown in Fig. 4.6 between temperature and ecosystem processes are low.

The experiments reported in this paper, taken together, suggest the hypothesis that the Pinatubo effect resulted from both the instantaneous effect of cooling on metabolism and the water and nutrient status of ecosystems prior to the cooling. For each site simulated, water and nutrient limitation at each time step reflected the preceding pattern of temperature anomalies and the lags specific to the ecosystem type being simulated. Thus, the Pinatubo anomaly reflected the history of temperature anomalies over the previous decade, as well as the instantaneous effect of the cooling. Global simulations with climate perturbations based on observed anomalies are now in progress to determine the broad-scale patterns that emerge from the complex site-specific responses (Braswell et al. 1995).

The modeled NEP perturbations from the temperature perturbation experiments range from $5-20 \%$ of steady state NPP. If spatially-coherent anomalies in climate produced responses of this magnitude, then global anomalies in the terrestrial carbon balance resulting from interannual climate variability could easily be of order several Pg C (assuming that global NPP is 40-60 Pg C; Schimel et al. 1995). Carbon anomalies in the 1-3 years following a climate anomaly could be as large as, or larger, and of opposite sign from the initial response, leading to the low time series correlations of temperature and biogeochemical anomalies observed in the temperature anomaly experiment. Changes of 5-20\% of NPP are, however, extremely 
difficult to detect given sampling variability using conventional techniques, and so observational detection will require care with methodology (Parton et al. 1995).

The role of lagged effects in the global carbon cycle

Several groups have attempted to quantify the effects of climate anomalies on terrestrial carbon storage using models compared to global $\mathrm{CO}_{2}$ observations. These efforts have often included the assumption that the effects of anomalous temperature and precipitation are instantaneous and have no lagged effects (e.g., Dai and Fung 1993). Other approaches cannot diagnose the mechanisms underlying any lagged effects because they use satellite observations (modified by climate data) to estimate NPP (Potter et al. 1993. Thompson et al. 1995), although lags may be present in the satellite observations. In Section 4.3 , we explore the potential for remote sensing measurements to provide information about the characteristic time scales of ecosystem response to temperature.

Observational time series studies suggest lagged effects. For example, the Mt. Pinatubo eruption appears to have caused an anomalous increase in terrestrial carbon uptake, apparent as a decrease in the growth rate of atmospheric $\mathrm{CO}_{2}$ (Braswell et al. 1995, Ciais et al. 1995a,b, Keeling et al. 1995, Schimel 1995b). This decrease in the growth rate, however, began before the eruption. Keeling et al. (1995) have speculated this may be a rebound from the warm years of the late 1980s, possibly reinforced by the Pinatubo cooling. This behavior is qualitatively consistent with some site-specific Century simulations. The direct post-Pinarubo effect may have occurred because of reductions in respiration with cooler temperatures that were larger than the decreases in NPP (Braswell et al. 1995). Keeling and colleagues have also suggested that, on decadal time scales, warming causes increases in terrestrial carbon uptake, but with a 2-year lag (Keeling et al. 1995,1996). This is a response consistent with a nutrient release- 
controlled response but not obviously with a Dai and Fung (1993) type of instantaneous physiological response of plants and decomposers. Because of the individualistic responses of the sites simulated in this study, we cannot yet determine whether Century's response is qualitatively consistent with Keeling et al.'s (1995) analyses, but the existence of significant lags in the model is a precondition for more comprehensive agreement.

Investigation of mechanisms leading to long-term equilibration and perturbation responses will increase understanding of processes tied to the biogeochemically "slow" components of ecosystems (woody biomass, detritus, and soil organic matter), processes that are difficult to study with experimental techniques. Such investigation will provide a crucial window into the components of terrestrial ecosystems that may govern long-term responses to changing climate, $\mathrm{CO}_{2}$, and other perturbations (e.g., nitrogen deposition) (Townsend et al. 1996; Chapter 3). For example, Hedin et al. (1995) suggest profound and long-lived effects of anthropogenic perturbations to nitrogen deposition. Ecosystem $\mathbf{N}$ inputs (deposition and biological fixation) are not well-known for much of the world (Holland et al. In press, Galloway et al. 1995), nor are they understood mechanistically. Nitrogen outputs are likewise not well-quantified globally (Hedin et al. 1995).

\subsubsection{Summary}

We hypothesize that water and nitrogen limitations of NPP are correlated at steady state because of the equilibration of water and nitrogen limitation of NPP that occurs through the control of carbon and nitrogen fluxes by the water budget. We further hypothesize that these correlations arise because of the structure of interactions amongst the water, carbon, and nitrogen cycles: they are a system-level property. As a corollary, ecological differences in resource use efficiencies, soil properties, and temperature should induce substantial quantitative 
variability in the water-nitrogen-carbon relationships, requiring that eventually, community and biogeographic processes be integrated into biogeochemical models. Many of the steady state patterns in Century arise through simulation of the nitrogen budget, rather than as a result of internal cycling. This is a unique feature of Century, and allows the mechanisms underlying resource interactions to be diagnosed explicitly. Most other current ecosystem models initialize N stocks based on observations (Potter et al. 1993), or iteratively, to force consistency with observed or assumed fluxes (Melillo et al. 1993).

These hypotheses and corollary are consistent with recent regional (Schimel et al. In press b) and global model results and some data, but require additional testing. Further. because the water, nitrogen, and carbon budgets respond to perturbations on different time scales, the responses of ET, nitrogen availability, and NPP during transient climate changes are not necessarily tightly correlated. Interannual variability in the terrestrial carbon cycle, as inferred from $\mathrm{CO}_{2}$ observations, may arise from complex multi-year interactions, and this complex behavior may become more evident as the spatial resolution and continental focus of atmospheric $\mathrm{CO}_{2}$ observations increases. Although Century suggests that the Mt. Pinatubo cooling could have caused a significant shift in terrestrial carbon exchange (consistent with observations), it also suggests that this effect was manifest through temperature effects on water relations, leaf area changes, and nutrient cycling, rather than via a simple effect of temperature on metabolism. The model further suggests that through lagged effects the magnitude of interannual changes in biogeochemistry should reflect climate patterns over several preceding years.

To detect either systematic $\left(\mathrm{CO}_{2}\right.$-caused: Friedlingstein et al. 1995, Fung et al. 1995; or N-caused: Townsend et al. 1996) or interannual changes, one must consider the possibility of lagged effects, and sampling designs and associated process studies should take into account 
both signal and noise arising from lagged changes to hydrological and biogeochemical processes (Fung et al. 1995). Detection of patterns and processes associated with water budget and biogeochemical lags will provide a window on the slower processes in ecosystem metabolism that may dominate global change effects on ecosystems in the future.

Finally, experimental evaluation of global ecosystem models is difficult (Schimel et al. In press $b$ ). Because the changes predicted for NPP, NEP, and respiration by the Century model are modest relative to local spatial sampling variability, observational validation will be difficult and may require careful multi-year monitoring with techniques insensitive to smallscale variability, such as flux measurements using aerodynamic techniques (Wofsy et al. 1993, Lenschow 1995), or will require inverse estimates from concentration fields (e.g., Ciais et al. 1995a,b). Experimental studies that directly probe the relevant mechanisms (i.e., by directly or indirectly changing transpiration, nitrogen availability, or carbon inputs via manipulative experiments) are critical; re-examination of existing studies of fertilization by $\mathrm{N}$ or $\mathrm{CO}_{2}$ may prove highly useful. Attribution of variation to specific mechanisms operating on multiple time scales will also require innovative measurements of nitrogen inputs, outputs, and internal cycling at large spatial scales.

Models with a wide range of formulations have all been able to reproduce observed variations in atmospheric $\mathrm{CO}_{2}$ or limited site-specific observations of NPP (e.g., Fung et al. 1987, Bonan 1993, Potter et al. 1993, Schimel et al. In press b) approximately equally well. Because site-specific measurements (e.g., of NPP) have high uncertainty resulting from local heterogeneity, it is difficult to reject ecosystem models using such data. However, global-scale process models make some very specific predictions about ecosystem-type differences in resource use efficiency and other robust measures of ecosystem physiology. These may be tested at large spatial scales using flux measurement techniques supplemented by critical 
nitrogen measurements. Resource use efficiency diagnostics of model performance are less subject to tuning than is NPP and also provides insight into model behavior under altered environmental forcing (VEMAP 1995). This study suggests that data sets providing, at an annual time scale, measures of NPP and water and nitrogen use efficiencies of ecosystems ranging from grasslands through savannas to forests could be extremely powerful in testing ecosystem models.

In the next section, we evaluate global observational evidence suggesting a significant role for lagged effects in the interannual response of the carbon cycle to climate consistent with results from theoretical and simulation studies presented above (also, Schimel et al. In press a). We emphasize global data sets because 1-2 Pg anomalies in atmosphere-terrestrial $\mathrm{C}$ exchange (e.g., Ciais et al. 1995b) represent small perturbations to the balance of global net primary

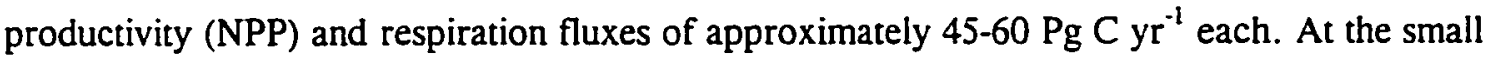
spatial scales of ecological dynamics, interannual variations of less than $25 \%$ (e.g., in NPP) are generally not detectable against local spatial variability (Parton et al. 1993). Moreover, few appropriate, long-term time series data exist for these variables (Parton et al. 1993, Goulden et al. 1996).

\subsection{Characteristic Responses of Global Terrestrial Ecosystems to Temperature Inferred from Satellite and Surface Data}

\subsubsection{Introduction}

A number of recent analyses using global observations of the concentration of atmospheric $\mathrm{CO}_{2}$, its isotopes, and $\mathrm{O}_{2}$ have demonstrated the importance of terrestrial processes in the global carbon cycle (Ciais et al. 1995a, Francey et al. 1995, Keeling et al. 1995.1996). This importance has been shown to extend to the issue of interannual variability (Keeling et al. 1995. Ciais et al. 1995b). A provocative model-based analysis suggests that interannual patterns 
of climate could have contributed to a net global terrestrial sink for $\mathrm{CO}_{2}$ (Dai and Fung 1993). Nevertheless, the mechanisms for interannual variability in terrestrial carbon exchange at the global scale are not clear.

A number of authors have speculated on causation, generally relying upon assumed effects of temperature on photosynthesis or respiration. This speculation is based on the wellknown physiological responses of plant and microbial processes to temperature (Farquhar et al. 1980, Parton et al. 1987. Holland et al. 1995), and has led to discussions of a scenario in which warming leads to an decrease in terrestrial carbon storage by increasing microbial respiration more than photosynthesis. This argument is based on the sort of responses implemented in. for example. the model of Sellers et al. (1996). but which imply near-instantaneous equilibration of physiological responses to environmental changes. "Instantaneous". in this context, implies time scales from minutes to less than a month; we will also use this term to refer to phenomena that appear in the data during the same averaging period, e.g.. one month. However, if processes at the ecosystem scale, such as feedbacks through soil water storage, plant phenology, or nutrient cycling (Rastetter et al. 1992, Shaver et al. 1993. Schimel 1995a) influence the response, we might expect the response of the biosphere to be lagged relative to environmental forcing (Keeling et al. 1995, Schimel et al. In press).

There has been considerable debate amongst ecologists over the relative roles of biophysical controls versus internal biogeochemical dynamics in governing productivity and carbon storage. The confusion arises from the fact that while broad spatial patterns of productivity are well-predicted from climatic variables (Rosenzweig 1968, Uchijima and Seino 1985), many (if not most) ecosystems respond to added nutrients (usually nitrogen) with increased productivity and carbon storage (Vitousek and Howarth 1991, Schimel 1995b). Distinguishing between these two paradigms is critical to establishing the credibility of models 
used in interpreting the observational record (Friedlingstein et al. 1995) and for evaluating scenarios of potential future changes (VEMAP 1995). If mechanisms acting through nutrient cycling are significant, it could add an additional set of time-scales of response to studies such as those of Sellers et al. (1996) and others (Bonan 1995. Henderson-Sellers 1995), and possibly alter conclusions relative to models based solely on rapidly-equilibrating processes.

\subsubsection{The observational record, 1982-1993}

We have assembled three extant data sets in order to independently evaluate the hypothesis (Schimel et al. In press a: Section 4.2) that the terrestrial carbon cycle exhibits complex behavior on interannual time scales in response to climatic perturbations. These measurements include two global remote sensing products and one set of site-network data: lower tropospheric temperature anomaly data from the Microwave Sounding Unit (MSU) (Spencer et al. 1990); Normalized difference vegetation index (NDVI) (Kidwell 1991) from the Advanced Very High Resolution Radiometer (AVHRR): and atmospheric $\mathrm{CO}_{2}$ concentration data from flask samples collected by the Climate Monitoring and Diagnostics Laboratory (CMDL) (Conway et al. 1994). In the following paragraphs we describe the characteristics of each data set, as well as the processing we applied to the data sets before the analysis.

The MSU temperature anomaly data are derived from passive microwave measurements (53.74 GHz) of lower tropospheric brightness temperatures (Spencer et al. 1990) which are based on the thermal emissivity of molecular oxygen. Global mean values are precise to within $0.01^{\circ} \mathrm{C}$, and individual gridpoint values to within $0.07^{\circ} \mathrm{C}$ in the tropics, and $0.15^{\circ} \mathrm{C}$ elsewhere. The MSU instrument is on board the NOAA polar orbiting satellites and it samples most of the earth twice per day, with a nominal footprint size of approximately $110 \mathrm{~km}$. The processed temperature anomaly (seasonal cycle removed) product is available on a monthly 
basis, registered to global 2.5 degree grid. The gridded data have been compared to surface thermometer-measured temperatures for various climate zones, and $R^{2}$ values are generally in the 0.94-0.98 range (Spencer and Christy 1992).

The NDVI data are produced from optical reflectance measurements by the AVHRR instrument which also resides on the NOAA polar orbiting satellites. The instrument measures outgoing radiance in five channels: red $(0.58-0.68 \mu \mathrm{m})$, near-infrared $(0.73-1.10 \mu \mathrm{m})$, and three thermal infrared bands. The Pathfinder AVHRR Land (PAL) product (Agbu and James 1995 ) is a global data set, with $8 \times 8 \mathrm{~km}$ spatial resolution and a 10-day compositing interval. The Pathfinder reflectances are derived from the "global area coverage" data that are spatially resampled on board the satellite. The data were co-registered to the MSU temperature fields by recompositing to one month and reprojecting the data onto a $2.5^{\circ}$ grid. We have also deseasonalized the data to create monthly anomalies by subtracting the mean annual cycle at each gridpoint (as is done with the MSU data).

The normalized difference vegetation index is equal to the difference between the reflectance measured in the red and near-infrared channels divided by their sum. Though NDVI is not a fundamental land surface or ecophysiological variable, there is a theoretical and experimental basis for the relationship between NDVI and a number of ecosystem parameters, including canopy photosynthetic efficiency, absorbed photosynthetically active radiation, stomatal conductance, and leaf area index (Tucker and Sellers 1986, Sellers 1987, Myneni et al. 1992b). In turn, these biophysical quantities are significantly associated with regional net primary productivity. The fundamental variables controlling NDVI are foliage density and chlorophyll content of leaves. Chlorophyll content is itself related to foliar nitrogen concentrations, and as such is an important link to nutrient cycling (Wessman et al. 1988). 
A number of factors not associated with land surface state can contribute to the signal, primarily atmospheric (Kaufman 1989) and bidirectional (Cihlar et al. 1994) effects (see also Chapter 5). Though the PAL data were corrected for ozone and Rayleigh scattering, the effects of aerosols remain. In addition, there are orbital drift effects and the related problem of instrument changeover. In large part, these numerous effects (except the latter two) are greatly reduced by recompositing and averaging the data (Singh and Saull 1988), as we have done in this study.

The $\mathrm{CMDL} \mathrm{CO}_{2}$ flask sample measurements are taken from a globally distributed network of sites. We used monthly averaged $\mathrm{CO}_{2}$ concentration data from 1979-1994 for two stations, Mauna Loa, Hawaii (MLO), and the South Pole (SPO) to represent Northern and Southern Hemisphere mean $\left[\mathrm{CO}_{2}\right]$, respectively. The concentration data for both stations were converted to growth rates by first-differencing the monthly signal, then removing the seasonal component. We deseasonaiized the data in a similar fashion to Keeling et al. (1989a) by fitting a 4th order harmonic function. Higher frequency components contribute little to the signal, and are accounted for by smoothing with a 3-month moving window. Removing the seasonal component of this data requires Fourier decomposition because the annual cycle of the point $\mathrm{CO}_{2}$ measurements represent a superposition of amplitude and phase for all the vegetation on the hemispheric land surface. We produced a global mean monthly growth rate time series by averaging the MLO and SPO curves.

Time series of global and hemispheric monthly mean temperature and NDVI anomalies were calculated from the satellite data (weighted by grid cell area). They are shown, along with the $\mathrm{CO}_{2}$ data, in Fig. 4.10. All three time series are approximately stationary, with zero mean, and relatively constant variance. A major exception, however, could be seen in the original NDVI time series. A period in the late 80 's to early 90 's is sharply delineated by two events: 

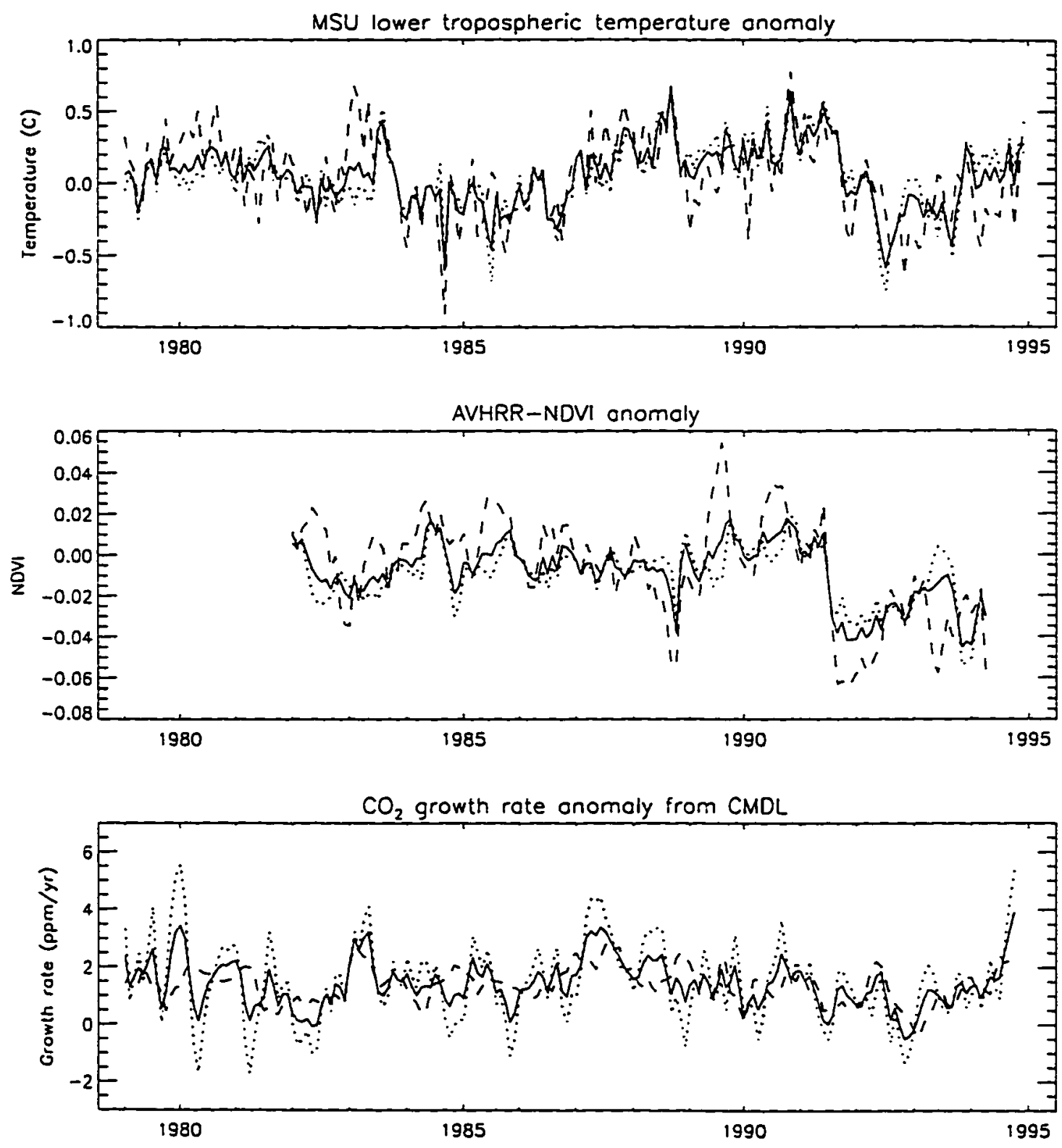

Figure 4.10 Global mean (solid), northern hemispheric mean (dotted), and southern hemispheric mean (dashed) anomaly time series of (a) lower tropospheric air temperature derived from the MSU; (b) normalized difference vegetation index from the AVHRR; and (c) atmospheric $\left[\mathrm{CO}_{2}\right]$ growth rate from flask measurements at Mauna Loa, Hawaii, and at the South Pole. 
the transition from NOAA-9 to NOAA-11 in November 1989, and the massive release of aerosols in June 1991 from the explosion of Mt. Pinatubo. The interval is less well defined for the MSU data because the processing incorporates nighttime temperature measurements (from even numbered NOAA satellite overpasses), and because the MSU is less sensitive to aerosols. It is likely that both temperature and vegetation experienced real changes after Pinatubo that are difficult to separate from aerosol artifacts. However, we have applied a simple correction: the period following the NOAA 9-11 changeover (excluding the year following Pinatubo) was shifted uniformly such that its mean anomaly was zero. This correction has the effect of reducing the magnitude of the discontinuity which is very apparent in the first difference of the data, while respecting the duration of the large Pinatubo aerosol effect (Dutton and Christy 1992).

\subsubsection{Methods}

\section{Correlation analyses}

With the three data sets, we calculated autocorrelograms and pairwise crosscorrelograms of global and hemispheric mean temperature, NDVI, and $\mathrm{CO}_{2}$ growth rate, defining the lags such that temperature always leads the other variables, and such that NDVI leads $\mathrm{CO}_{2}$ growth rate. In principle, all the variables could have non-zero correlations for both positive and negative lags. However, the effects we consider shall be larger and more direct on the time scales considered here. We will focus the discussion on time scales over which ecosystems can realistically produce lagged effects ( $1-4$ years, as suggested by the Century model).

The autocorrelations (Fig. 4.11) are monotonically decreasing without periodicities for lags less than four years for the $\mathrm{CO}_{2}$ data, for the temperature anomaly, and for the northern 
hemispheric NDVI anomaly. Beyond these time scales, there exists emerging oscillatory behavior which likely originates from internal climate cycles. For example, both temperature and $\mathrm{CO}_{2}$ growth rate appear to have a 7-8 year cycle. The southern hemispheric NDVI shows a significant 4-5 year periodicity which is probably associated with the El Niño-Southern Oscillation (ENSO). Myneni et al. (1996) have demonstrated the existence of an ENSO signal in NDVI data, arising from precipitation-driven changes in vegetation productivity in predominately semi-arid regions of the Southern Hemisphere.

In Fig. 4.12a, a significant relationship between $\mathrm{CO}_{2}$ growth rate and temperature can be seen, centered around a two-year lag. Keeling et al. (1995) suggested that such a delayed response could have been responsible for a low $\left[\mathrm{CO}_{2}\right]$ anomaly that began before the Pinatubo eruption, and continued downward until late 1993. We suggest that complex terrestrial responses, stimulated by temperature changes, result in either enhanced plant production, reduced heterotrophic respiration, or both, such that global integral net ecosystem production is positive approximately two years after a warm anomaly. Conversely, a cool anomaly should lead to enhanced terrestrial uptake.

Turning to the relationship between temperature and NDVI (Fig. 4.12b), there is no immediate (0-1 year lag) global or southern hemispheric response to temperature anomalies. and only a weakly significant correlation for the Northern Hemisphere at lags of less than six months. Hence, it may be true that if temperature changes produce immediate effects, then they are invisible to optical remote sensing, i.e., they do not result in increased foliage amount or nitrogen content. There is also, however, no evidence in the global or hemispheric means of any biogeochemical "memory" of past temperature changes for lags less than 3-4 years. It is likely that temperature effects on ecosystems are highly localized and that there are spatiallyexplicit lagged and instantaneous effects that offset one another in the hemispheric or global 


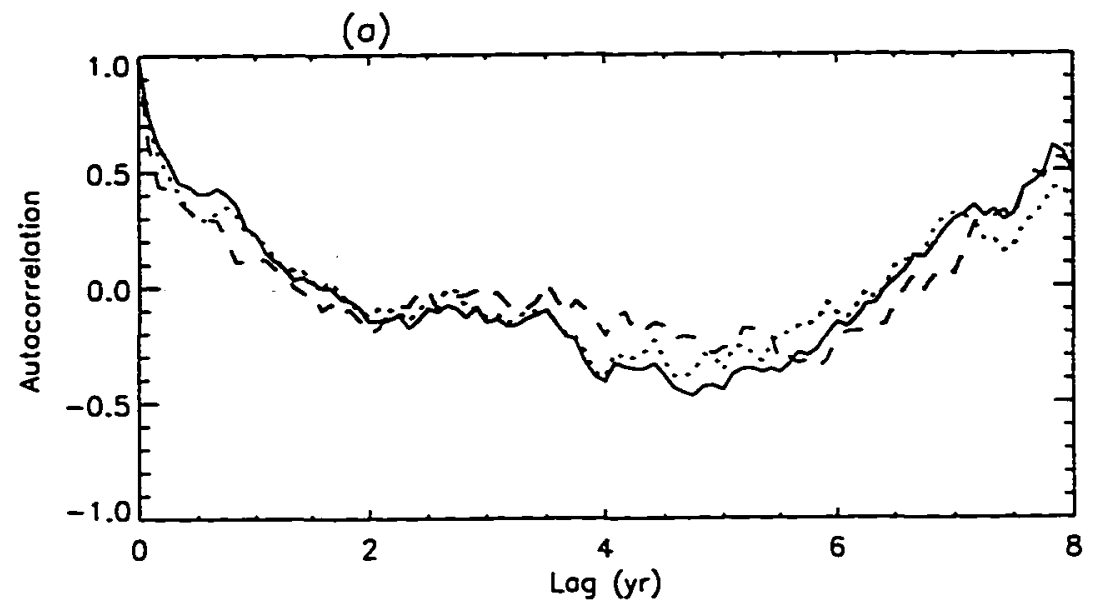

(b)

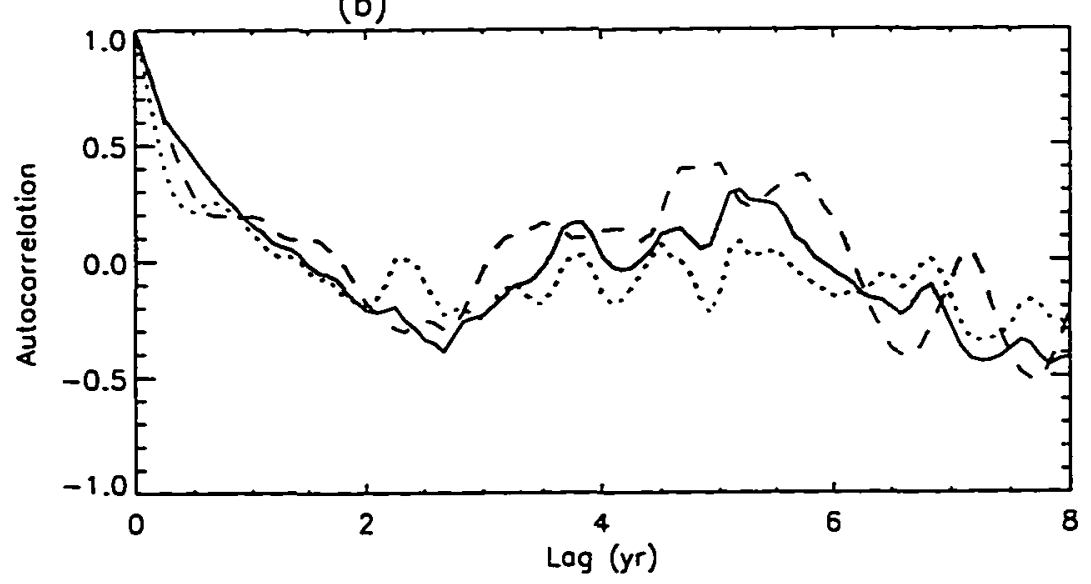

(c)

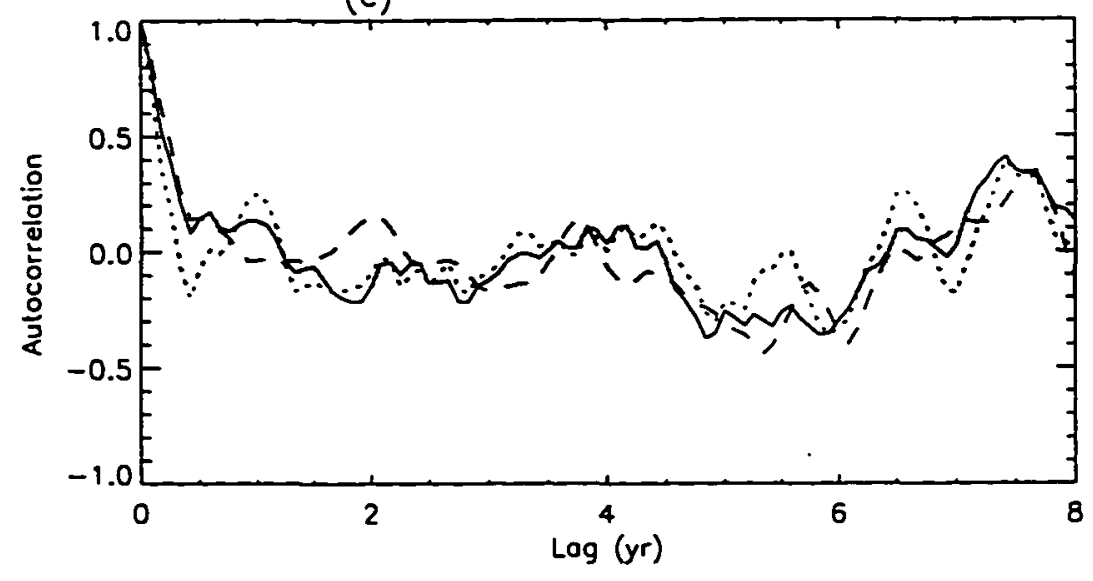

Figure 4.11 Autocorrelation function of the time series in (4.10): (a) temperature; (b) NDVI; and (c) $\left[\mathrm{CO}_{2}\right]$ growth rate. The shaded region indicates autocorrelations that are not significantly different from zero $(\mathrm{P}<0.05)$. 
(a)

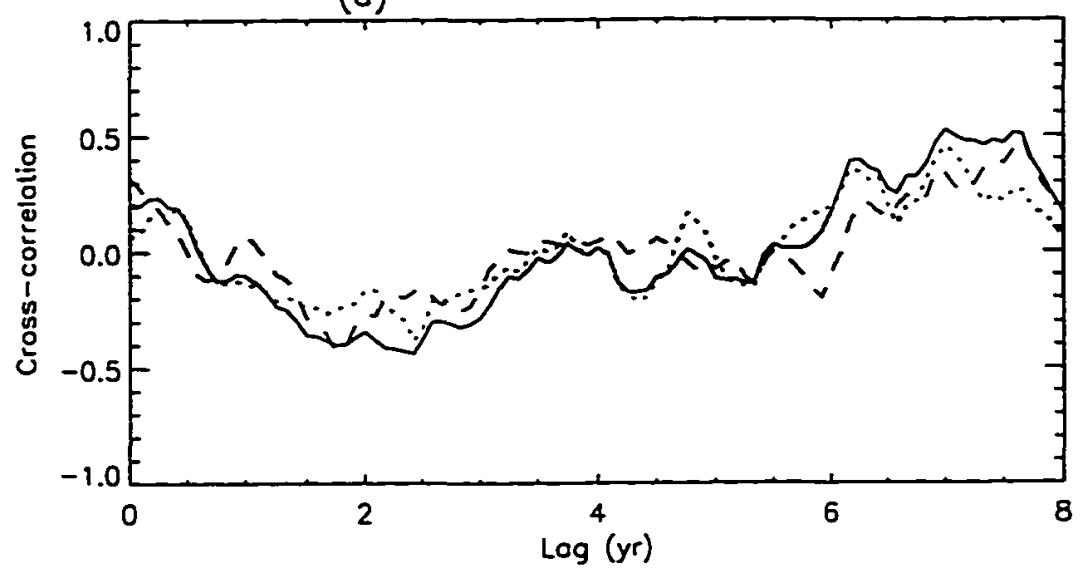

(b)

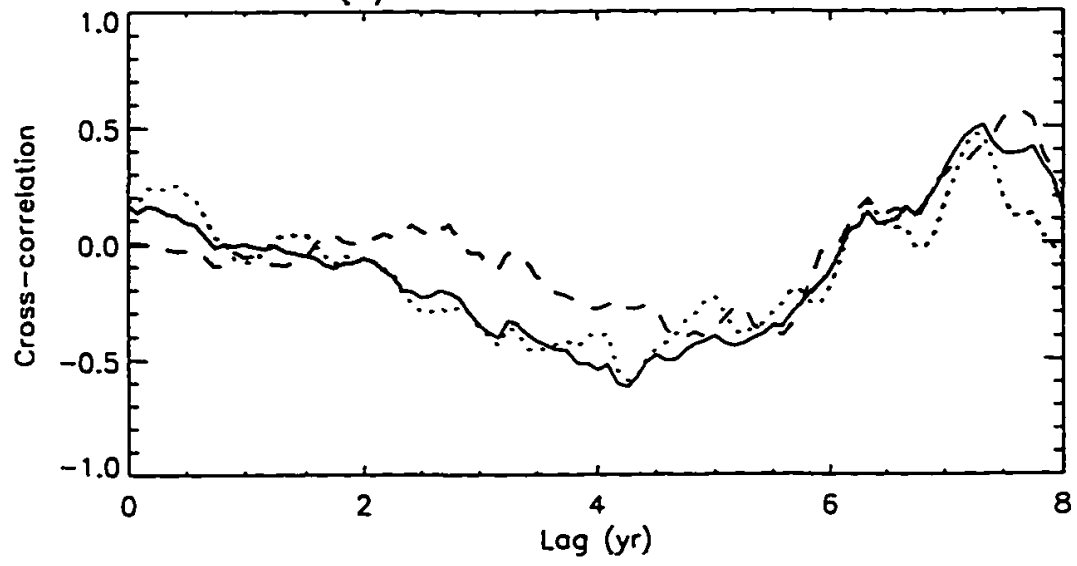

(c)

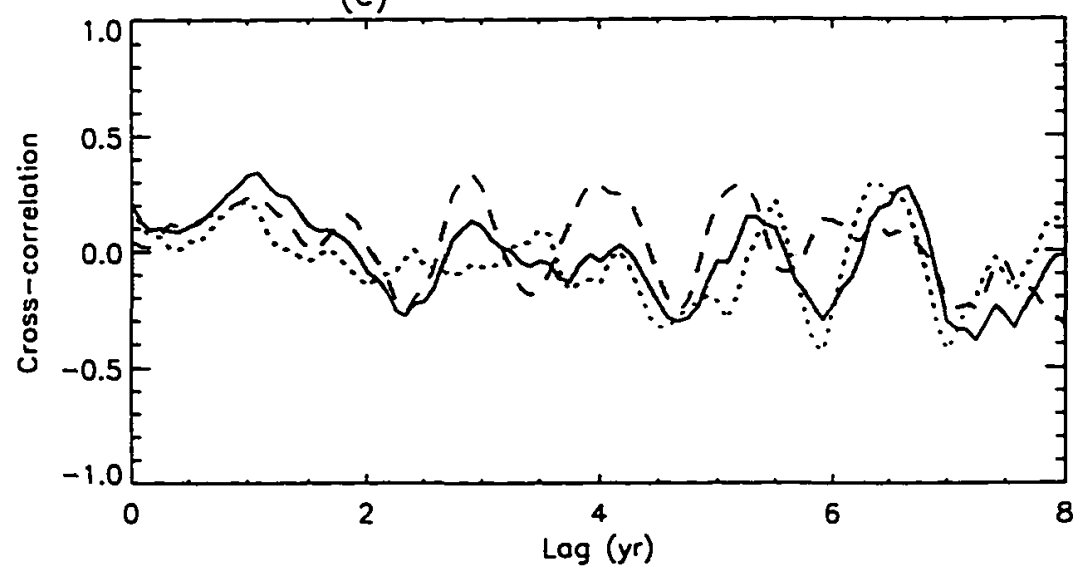

Figure 4.12 Cross-correlation functions: (a) lagged temperature vs. $\left[\mathrm{CO}_{2}\right]$ growth rate; (b) lagged temperature vs. NDVI: (c) lagged NDVI vs. $\left[\mathrm{CO}_{2}\right]$ growth rate. 
mean. We will investigate this possibility below.

We see very little significant interaction between lagged $\mathrm{NDVI}$ and $\mathrm{CO}_{2}$ growth rate. particularly for lags less than 4 years, and particularly for the Northern Hemisphere record. There is only the weak anti-correlation at lags of approximately two years. If warming causes a delayed terrestrial response that is causally related to a delayed decline in $\mathrm{CO}_{2}$ growth rate. then why is there no instantaneous positive correlation? We suggest two possibilities: first, internal terrestrial mechanisms (i.e., those related to dead organic matter dynamics) are the primary factors related to the interactions seen in Figs. 4.11a and 4.11b: second, as mentioned above, zero-lag correlations exist, but are balanced by anticorrelations that occur elsewhere. The latter possibility can not be investigated given the data at hand, but the former is entirely consistent with the Century results of Schimel et al. (In press a), in which respiration anomalies dominated the net ecosystem $\mathrm{CO}_{2}$ flux anomalies.

Because the mean global and hemispheric patterns seen in Figs. 4.10-4.12 arise from complex interactions at the local and regional scale, we have calculated NDVI-temperature correlograms (cf. Fig. 4.12b) for each gridpoint. Figure 4.13a shows the lag time at which the correlation has the largest positive value. Similarly, the time at which the anticorrelation is greatest is shown in Fig. 4.13b. For both these maps we have restricted the analysis to timescales of less than four years. Taken together. Figs. 4.13a and 4.13b allow partial visualization of the correlograms at each gridpoint. The ecosystems that likely give rise to the global and hemispheric mean response (Fig. 4.12b) can be seen to lie in the low-to-mid latitudes. It is apparent that instantaneous cross-correlations exist, but that they cancel in computing the global mean. Many arid systems have an instantaneous negative vegetationtemperature relationship, and that high-latitude systems tend to have longer delay times for anticorrelations. Both maps show features that strongly resemble biome distribution boundaries. 
Time of Maximum Cross-correlation Between NDVI and Lagged Temperature
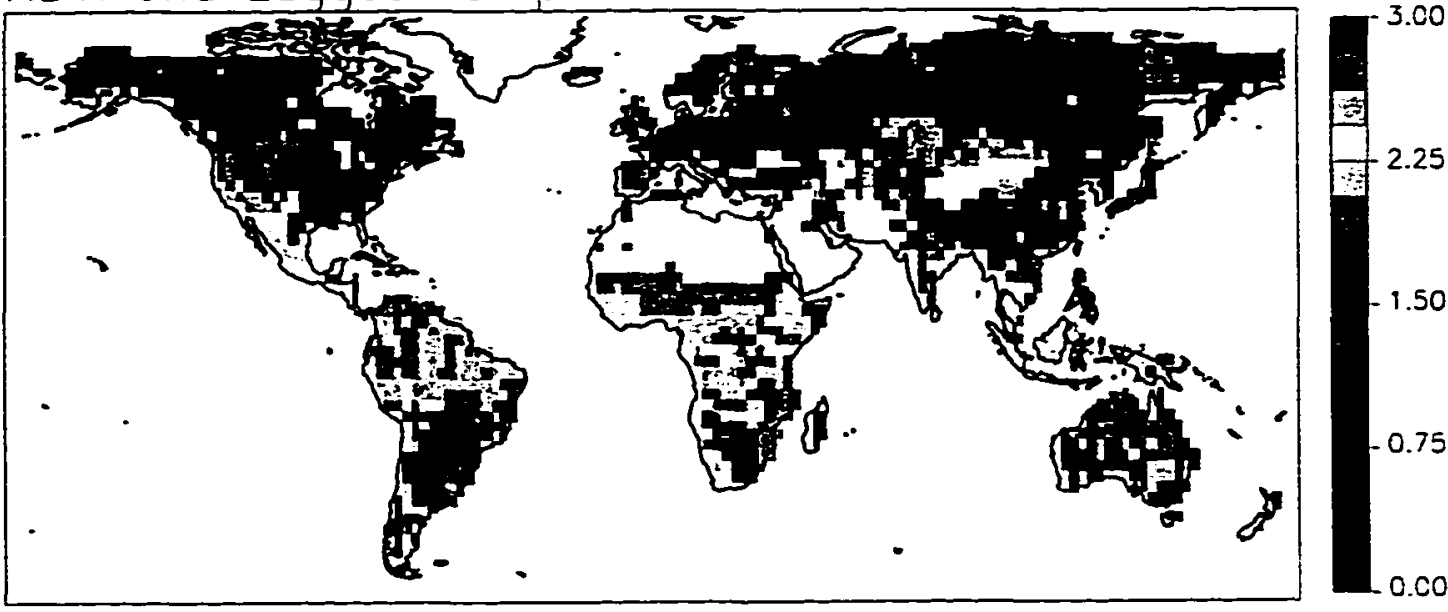

Time of Minimum Cross-correlation Between NDVI and Lagged Temperature
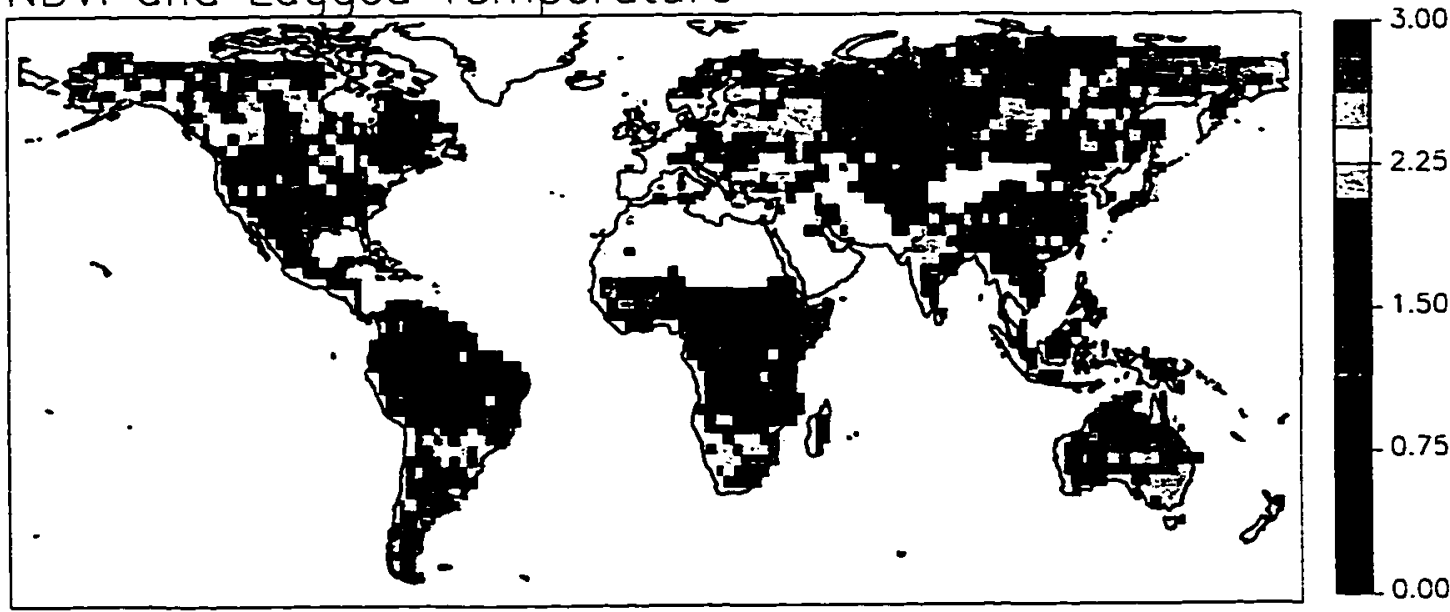

Figure 4.13 (a) Spatial distribution of the lag time at which lagged temperature is most positively correlated with NDVI. (b) Same as (a) except showing the lag time at which the two variables are most negatively correlated. This analysis considers lag times less than four years to avoid picking up variability due to natural climate cycles. 
This suggests coherent terrestrial responses and further emphasizes the importance of biomeand species-specific plant and soil properties (Schimel et al. In press b).

Up to this point we have used simple analyses of time-series (averaged over space or grid cell-by-grid cell) to extract correlation information from the global data. It is likely, however, that there are time-dependent effects in the satellite measurements (especially with the AVHRR) which confound interpretation of the interannual signal. While spatial and temporal averaging reduces errors associated with small scales of spatial and temporal variability, low frequency contamination remains, and is principally associated with large-scale atmospheric composition (e.g., aerosols and water vapor) and orbital drift (mentioned above). Though we have applied a defensible (C.J. Tucker. Personal communication) bias correction to correct for the NOAA 9-11 changeover, there is no way to independently evaluate the veracity of year-toyear changes in NDVI that are typically 0.01-0.06 NDVI units (less than 10\% of the possible range).

An alternative method: inferring temporal dynamics from lagged spatial correlations

A different, and somewhat more detailed, statistical analysis was performed on the geographically referenced temperature and NDVI data. In addition to the question of the existence of a reliable interannual signal in the AVHRR data (discussed above), the highly localized behavior apparent in both data sets produces difficulty in interpreting the temperaturegreenness cross correlations at the global/hemispheric scale (i.e., Fig. 4.12b vs. Fig. 4.13). Thus, we examined their correlations spatially. The data were aggregated into global land cover classes according to the map of DeFries et al. (1995); within each class, and for each year, multiple linear regressions of NDVI anomaly against temperature anomaly were 
performed. The dependent variable in the regression is "current" NDVI, and the independent variables are temperature in the current year, temperature in the last year, and so on, i.e.,

$$
\mathbf{Y}=\mathbf{X b}+\varepsilon
$$

where $\mathbf{X}$ is a matrix constructed from the temperature anomalies in year $t, t-1, t-2$, etc., and $\mathbf{Y}$ is the dependent variable, NDVI. We assume that within-class pixels are essentially replicate measurements, reasoning that across-biome variability tends to be greater than interannual variability so errors due to low signal-to-noise are minimized. In this manner, regression coefficients $b_{i}$ (slopes) are retrieved for each biome and for each year that are related to current and lagged ecosystem response to temperature, but are not affected by year-to-year changes in the mean. This technique is an alternative to traditional time-series analysis (above), facilitated by the assumption that spatially adjacent ecosystems, which are also morphologically and physiologically similar, manifest similar responses to temperature.

The pooled within-biome data, which we treat as multiple samples, are not independent because of their proximity to one another. Therefore, we accounted for the spatial correlation in the data by estimating regression coefficients using the method of generalized least squares:

$$
\hat{\mathbf{b}}=\left(\mathbf{X}^{\prime} \Omega^{-1} \mathbf{X}\right)^{-1} \mathbf{X}^{\prime} \Omega^{-i} \mathbf{Y}
$$

where $\Omega$ is the variance covariance matrix of the residuals $\varepsilon_{i}$ in (4.7) taken from estimated semivariograms of $\varepsilon$ for each biome class. In addition to spatial correlations, there potentially exists autocorrelation between the independent (lagged) temperature variables, so we performed a "test to remove $\mathrm{n}$ variables" on the significance of the $\hat{b}_{i}$ coefficients. Thus, the resultant 
regression model contains the combination of terms (if any) corresponding to significant $(P<0.05)$ 0-year, 1-year, or 2-year lags. Finally, the coefficients were standardized to path coefficients $\hat{\mathbf{p}}$ by the transformation $\hat{p}_{i}=\hat{b}_{i} S_{i} / S_{\mathbf{Y}}$ (for $i=0,1,2$ ), where $S_{i}$ denotes the standard deviation of the $i$ th lagged independent variable $X_{i}$, and $S_{\mathrm{Y}}$ is the standard deviation of the vegetation index values $Y$. Thus we present coefficients (Fig. 4.14) that are a "commensurate measure of response" (Selvin 1995).

Global and hemispheric means of the standardized regression coefficients are shown in Fig. 4.15. This calculation was performed on annual-mean data, so we present one plot each for 0,1 , and 2 year lagged responses. The monthly data were integrated in such a way as to significantly weight growing season anomalies: negative absolute NDVI values were truncated to zero to eliminate ice, snow, and bare ground; and negative temperature values were truncated to zero (biotemperature). There is considerable temporal variability in the magnitude and sign of the temperature response that could not have been observed in the time-series analyses (above). This is consistent with the complexity observed by Schimel et al. (In press a). The zero-lag coefficients correspond to global warming and cooling associated with El Nino, but appear to be dominated by the Pinatubo effect (in 1992). It is not clear if the Pinatubo signal is an artifact, especially because there does not appear to be a similar pattern associated with the 1982 eruption of El Chichon, which had a greater effect on aerosol optical depth (Dutton and Christy 1992). The 1991-1993 period also corresponds to a sustained ENSO event, and it is possible that there were significant interactions between these two unusual climatic events. Nevertheless, the zero-lag (immediate effect) coefficients contain the bulk of the effects which contaminate the land-surface signal: interannual variability in the atmosphere, instrument calibration, and satellite orbital drift. These effects are likely to not be present in the lagged coefficients. 

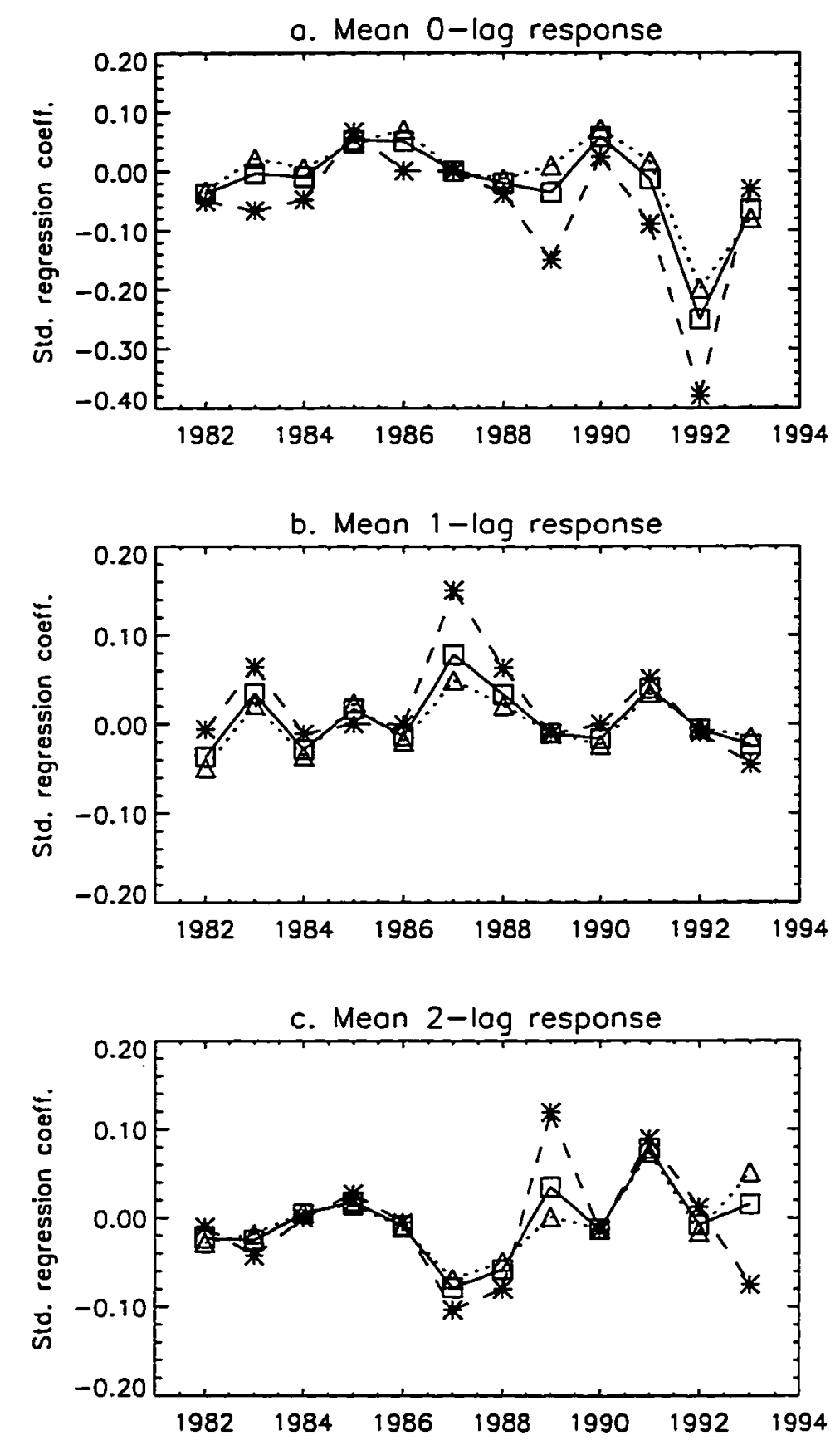

Figure 4.14 Standardized regression coefficients of a multiple linear regression of current NDVI on present (zero lag) and past (one and two year lags) temperature. The regressions were performed spatially, and within biome, for each year using annual mean data. The means were corrected to exclude snow cover and below-freezing temperatures. There is one regression coefficient for each lag time $\left(N_{\text {lag }}\right)$, and they are globally averaged, keeping only the significant correlations. (a) Zero lag; (b) One year lag; (c) Two year lag. The coefficients represent the percent change in greenness associated with a percent change in temperature that occurred $N_{\text {lag }}$ years ago. 
Figs. $4.15 \mathrm{~b}$ and $4.15 \mathrm{c}$ show the results of the spatial-temporal analysis for one-year lagged effects and two-year lagged effects. These results are consistent with the above crosscorrelation calculation (Fig. 4.12), and with the notion that terrestrial mechanisms give rise to delayed $\mathrm{CO}_{2}$ response to climate. The magnitudes of the two-year lagged responses are not less than the zero or one year lagged response. Patterns emerge that are likely related to large-scale climate variability (e.g., El Niño), modulated by the spatial distribution of the temperature anomaly. For example, 1985 was an anomalously cool year for most of the globe (for most ecosystems it is second only to the Pinatubo cooling), and there is a corresponding dip in the 2year response coefficient in 1987 (Fig. 4.15b). In contrast, 1989 was a very warm year, and there is a corresponding peak in the coefficient in 1991, but the tropics experienced unusually cool temperatures during half of that year because of Pinatubo.

\subsubsection{Discussion}

The correlations of temperature and $\mathrm{CO}_{2}$ growth rate on time-scales less than one year are likely direct effects of temperature on respiration, as suggested in Schimel et al. (In press a). Warm anomalies lead to increased terrestrial $\mathrm{CO}_{2}$ uptake and high NDVI with 1.5-3.0 year lags. Nutrient release during warm years lead to increased carbon uptake with $1.5-3.0$ year lags, and conversely, cool anomalies lead to reduced nutrient release and lagged reductions in carbon uptake. Further, the turnover time for deep soil moisture that is accessible to microbes and plants can be long (J. Famiglietti, personal communication). An anomalously warm year will have increased evapotranspiration, and thus a deficit in soil water can be carried over from the end of one growing season to the beginning of the next.

The coordinated lags in NDVI suggest that increased plant productivity removes additional $\mathrm{CO}_{2}$ from the atmosphere 1.5 to 3 years after warm anomalies, while absorbing 
lower-than-average amounts after cold anomalies. In addition, it is possible that effects of changing heterotrophic respiration are significant, and not invisible to remote sensing (for lags $\geq 1$ year) because they can propagate into changes in foliage quality and amount. These observational analyses, together with recent theoretical and simulation studies (Pastor and Post 1993, Schimel et al. In press a) suggest that biogeochemical mechanisms are key components of the dynamic response of the terrestrial biosphere to climate variability and, likely, change. The changes in the $\mathrm{CO}_{2}$ growth rate reflect changes to global NEP. Since temperature affects both plant and microbial physiology similarly, a change in NEP with temperature reflects the small asymmetry in the temperature responses of autotrophs and heterotrophs. Release of nutrients leading to delayed responses in NPP can have a large effect on NEP as the lagged NPP responses would not be accompanied by a simultaneous increase in microbial respiration.

While the available global biogeochemical and ecological data do not directly implicate nutrient cycling, the lags in the temperature, $\mathrm{CO}_{2}$, and NDVI correlations are consistent with the time-scales of biogeochemical regulation and inconsistent with the time-scales of instantaneous physiological adjustment. The spatial patterns of temperature-NDVI correlations indicate that, although global correlations exist, they are a composite signal from individualistic responses at different locations. As suggested in modeling studies (Bolker et al. 1995), mechanistic understanding must account for the differing sensitivities and time-scales of response in different ecosystems and by different plant species (Canham et al. 1995).

\subsection{Summary}

Within ecology, there has been considerable confusion and controversy over the largescale significance of limitation of net primary production (NPP) by nutrients vs. biophysical quantities (e.g., heat, water, sunlight) with considerable evidence supporting both views. The 
Century model, run to a quasi-steady state condition, predicts "equilibration" of water with nutrient limitation, because carbon fixation and nitrogen fluxes (inputs and losses) are controlled by water fluxes, and because the capture of nitrogen into organic matter is governed by carbon fixation. Patterns in the coupled water, nitrogen, and carbon cycles are modified substantially by ecosystem type or species-specific controls over resource use efficiency (water and nitrogen used per unit NPP), detrital chemistry, and soil water holding capacity.

We examined the coupling between water and nutrients during several temperature perturbation experiments. Model experiments forced by satellite-observed temperatures suggest that climate anomalies can result in significant changes to terrestrial carbon dynamics. The cooling associated with the Mt. Pinatubo eruption aerosol injection may have transiently increased terrestrial carbon storage. However, because processes in the water, carbon, and nitrogen cycles have different response times, model behavior during the return to steady state following perturbation was complex and extended for decades after 1- to 5-year perturbations. Thus consequences of climate anomalies are influenced by the climatic conditions of the preceding years, and climate-carbon correlations may not be simple to interpret.

Finally, we evaluated our model-based hypothesis with global data. Direct atmospheric measurements of $\mathrm{CO}_{2}$ concentrations are available over a period commensurate with coarse resolution satellite observations of lower tropospheric temperature anomaly and vegetation index (the past 12-15 years). We computed simple cross-correlations as well as a more detailed transfer-function model based on within-biome patterns of temperature response. Results from the analysis of these three global data sets suggests that interannual net biosphere-atmosphere exchange of carbon dioxide is determined by terrestrial biogeochemical mechanisms which operate at local-to-regional scales, and which yield significant temporally lagged responses to climate variability. 
Based on the observed dynamical behavior of the Century model and analysis of global data, we conciude that internal ecosystem dynamics lead to climate- $\mathrm{CO}_{2}$ relationships that are not "instantaneous". This result is inconsistent with the view that terrestrial carbon exchange is dominated by direct biophysical controls. Coupled climate system models are being developed, with a particular emphasis on the relationship between climate and $\mathrm{CO}_{2}$ variability (e.g., http://www.cgd.ucar.edu/csm). We make the case that in order to predict annual- to decadalscale changes in atmospheric $\mathrm{CO}_{2}$ concentrations. the dynamics of terrestrial processes such as net primary production and heterotrophic respiration must be represented, particularly with respect to water and nutrient storage and interactions that can cause complex and multi-year responses to climate. 


\section{CHAPTER 5}

\section{INTEGRATING REMOTE SENSING AND PHYSICAL MODELING TO ESTIMATE THE STATUS OF TERRESTRIAL VEGETATION}

\subsection{Introduction}

Moderate resolution satellite remote sensing provides the only means of directly observing the entire surface of the Earth at regular spatial and temporal intervals. In the context of this work, moderate resolution refers to measurements where the smallest unit of landscape is on the order of approximately one square kilometer, and the temporal interval is approximately daily-to-weekly. Optical measurements of reflectance in the solar spectrum are particularly useful for estimating the status and level of activity of terrestrial ecosystems. There are existing and proposed satellite instruments that provide this type of data. These measurements yield information about the behavior of the land biosphere as an important component of global biogeochemical cycles and about its interactions with the physical climate system. Satellite-derived information about the land surface can be used to make predictions about the changing composition of the atmosphere (using biogeochemical models) and about changing climate (using general circulation models).

The quantities that can be estimated using remote sensing measurements include bulk biophysical parameters (e.g., albedo), which are important for characterizing the balance of energy and water fluxes; morphological parameters (e.g., leaf area), which are important determinants of atmospheric momentum transfer; and optical parameters (e.g., leaf reflectance), which are related to vegetation health and site quality (water and nutrient status). 
Perhaps the most important biophysical quantity for ecological modeling is the amount of photosynthetically active radiation absorbed by plant canopies (APAR), which we will focus on in this chapter. All the retrievable characteristics mentioned above are related to terrestrial productivity, and as such can be used to constrain estimates of net photosynthesis and other biogeochemical fluxes.

Three of the principal goals of biogeochemical remote sensing science as presented by the EOS Science and Mission Requirements Working Group (NASA 1984) are to: "(I) Understand the biogeochemical cycling of carbon, nitrogen, phosphorus, sulfur, and trace elements; (2) Determine the global distribution of biomass and what controls both its heterogeneous distribution in space and its change over time; and (3) Determine the global distribution of gross primary production and respiration by autotrophic and heterotrophic organisms and the annual cycle and year-to-year variation of these processes. "With respect to the terrestrial biosphere, progress on any of these goals will benefit from the remote observation of vegetation type, amount, or status. Thus, an improved understanding of how terrestrial ecosystems are coupled to climate and global biogeochemistry - the common subject of the preceding chapters - can result from improved algorithms for retrieval of land surface characteristics.

In this chapter we discuss methods for extracting biophysical parameters from satellite optical remote sensing measurements. We focus on the information contained in the spatial and temporal patterns of continental-to-global scale optical reflectance data. There are essentially two broad categories of methods for interpreting coarse spectral and spatial data for terrestrial biophysical applications. First, the multi-channel reflectance measurements can be mapped into a scalar function called a vegetation index (VI), the values of which are empirically related in turn to a land surface variable. usually via field observations. In this approach. it is first 
necessary to have a VI that is insensitive to factors unrelated to the state of the land surface. and then to have an understanding of causes of variability in the VI-biophysics relationships. In the second approach, a physical model of canopy reflectance is inverted, either analytically or numerically, to estimate vegetation canopy (input) parameters. This method also requires consideration of factors which contaminate the land signal, and it is generally more computationally intensive. There are a number of unique advantages to inverse modeling, however, which will be discussed later.

In the next section (5.2), concepts relating to empirical approaches (using correlations between ecosystem variables and VIs) are discussed. There have been various assumptions made in the literature about the meaning and applicability of these relationships, but a number of theoretical and intuitive interpretations of vegetation indices are available. Qualitatively, higher values of a VI indicate more vegetation activity which is usually due to a greater density of foliage, or more productive plants within an ecosystem. Thus, the value of a vegetation index is often called "greenness". The most sensible framework for using vegetation indices to address ecological problems includes consideration of the physical meaning of the VI at the spatial and temporal scales of interest. This consideration should extend to accounting for the influence of biome-specific characteristics, including soil type, plant architecture, and the state of the canopy itself.

The principal contribution of this chapter is the inversion of a physical radiative transfer model with optical reflectance (from a global data set) to estimate land surface characteristics (Section 5.3). In particular, we emphasize the importance and usefulness of the dependence of reflectance on the geometrical configuration of source and sensor. The spectral information is also used, but in a different manner than with Vls. This is because, in model inversion, the reflectance measured in each channel (in a particular wavelength band) does not 
have to be combined into a single number. Instead. all measurements, sampled at unique wavelengths or with unique geometries, can be used as dependent variables. Thus, the information content of the data is exploited in an efficient manner. Difficulty arises in model inversion as a result of the weighing the balance between the number of parameters that are desired, and the dimensionality of the data (a function of the number of measurements and their degree of independence). The latter is never precisely known, but can be addressed within the modeling framework (e.g., Privette et al. In press).

The utility and feasibility of vegetation reflectance model inversions was introduced by Goel and Streibel (1983). Since that time, successful radiative transfer model inversions have been performed using various types of field and laboratory radiometer data. These experiments have typically utilized a large number of sampling geometries under controlled conditions. Perhaps because of sparse of sampling, difficulties with atmospheric contamination, and issues related to balancing model complexity with invertability, real satellite data has rarely been used in this context. Two studies have successfully used satellite observations of one area for a limited period (Flasse et al. 1993. Privette et al. In press). Section 5.3 is an extension of work by Privette et al. (In press) who demonstrared the importance of ancillary biophysical information about the target to the accuracy of parameter retrieval.

\section{Radiometric Calibration}

Interpreting remote sensing data begins with an understanding of the physical quantities that are involved. There is a great deal of literature pertaining to radiometric calibration, i.e., relating the "signal" of a detector to variables (e.g., reflectance) that are meaningful for inferring characteristics of the surface. We will follow the notation of Wyatt (1978), and present the most basic concepts. All the terms we introduce are a function of wavelength but it 
is assumed they are defined over some finite wavelength interval and we avoid explicit spectral dependence in the notation for convenience.

Satellite optical remote sensing instruments measure radiation in the visible and nearinfrared (NIR) wavelengths that originates at the sun, interacts with the atmosphere and the Earth's surface, and is transmitted back through the atmosphere to be detected by the sensor (Fig. 5.1). The flux of solar radiation, or irradiance $\left(\mathrm{W} \mathrm{m}^{-2}\right)$, at the surface can be expressed as:

$$
E_{s}\left(\mathbf{r}^{\prime}\right)=E_{s, 0} \tau_{A} d^{2} \cos (\theta)
$$

where $E_{5,0}$ is the mean exoatmospheric irradiance, $\tau_{A}$ is the atmospheric transmissivity, $d^{2}$ is the squared ratio of the Sun-Earth distance to the mean distance, and $\theta$ is the solar zenith angle. Use of Equation 5.1 requires knowledge of the day of year, the coordinates on the Earth, the atmospheric conditions, and the time of day.

The fundamental physical quantity useful for describing reflected radiation is radiance $L\left(\mathrm{~W} \mathrm{~m}^{-2} \mathrm{sr}^{-1}\right)$. Radiance is defined as the flux of radiant energy $\Phi\left(\mathrm{W} \mathrm{m}^{-2}\right)$ per unit solid angle $\omega$ (of the sensor) per unit area $A$ projected in the viewing direction (at the source), i.e.,

$$
L\left(\mathbf{r}, \mathbf{r}^{\prime}\right)=\frac{d^{2} \Phi}{d A \cos (\theta) d \omega}
$$

This quantity corresponds to "brightness"; the energy flux (and thus the number of photons) collected by a sensor integrates over the field of view and solid angle subtended by the detector. Sensors are designed to produce an output in digital number (DN; photon counts) that 


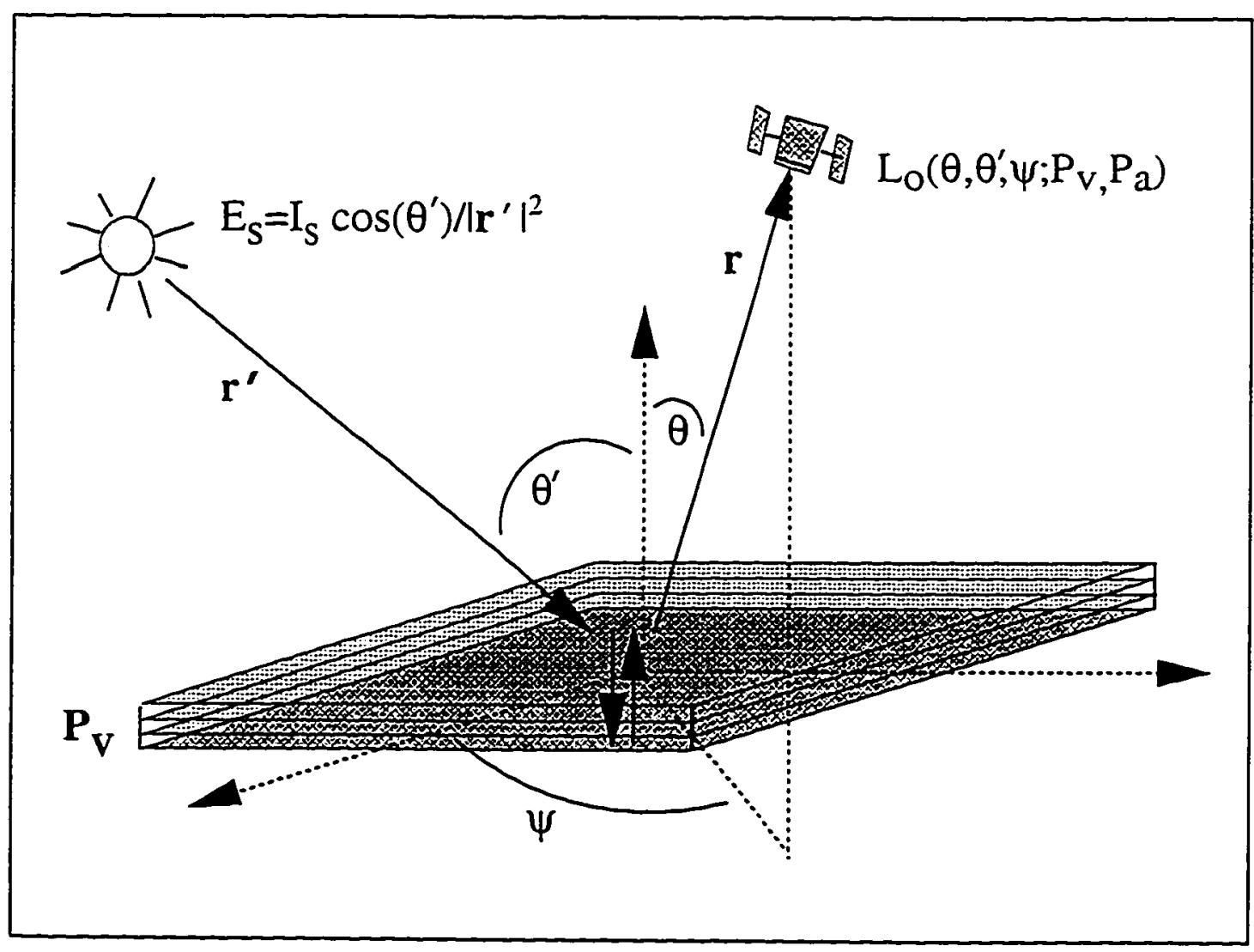

Figure 5.1 Schematic depicting the configuration of sun and sensor in the observation of bidirectional reflectance of a target (at the origin) from a satellite. 
is directly proportional to the radiance: $L_{0} \approx(D N-\beta) / \gamma$, where $\beta$ is the offset (count), and $\gamma$ is the gain (count $\mathrm{W}^{-1} \mathrm{~m}^{2} \mathrm{sr}$ ).

The quantity that is usually contained in calibrated optical remote sensing data is the bidirectional reflectance factor (BRF) $R\left(\mathbf{r}, \mathbf{r}^{\prime}\right)$, though it is commonly referred to as reflectance. The BRF is the ratio of measured radiance to that of a hypothetical perfect diffusing panel $L_{p}$ at the surface.

$$
R\left(\mathbf{r}, \mathbf{r}^{\prime}\right)=\frac{L_{o}}{L_{p}}=\frac{\pi L_{o}}{E_{s}}
$$

where $E_{s}$ is given by Equation 5.1; it appears in the expression because of Lambert's cosine law and the fact that $L_{p}$ has no solid angle dependence:

$$
E_{s}=E_{p}=\int_{2:=} L_{p} \cos (\theta) d \omega=\pi L_{p}
$$

Combining Equations 5.1 and 5.4, we arrive at a simplified expression for the calibration of the BRF, i.e.,

$$
R_{\Lambda}\left(\mathbf{r}, \mathbf{r}^{\prime}\right)=\frac{\pi L_{o . \Lambda}\left(\mathbf{r}, \mathbf{r}^{\prime}\right)}{E_{s, 0.1} \tau_{A} d^{2} \cos (\theta)}
$$

where we have noted explicitly the quantities that represent integral values over a wavelength band $\Lambda$ defined by the interval $\Lambda=[\lambda, \lambda+\Delta \lambda]$. The BRF takes on possible values from zero to infinity, but for the Earth's surface it is nearly always less than one because of the absorbing 


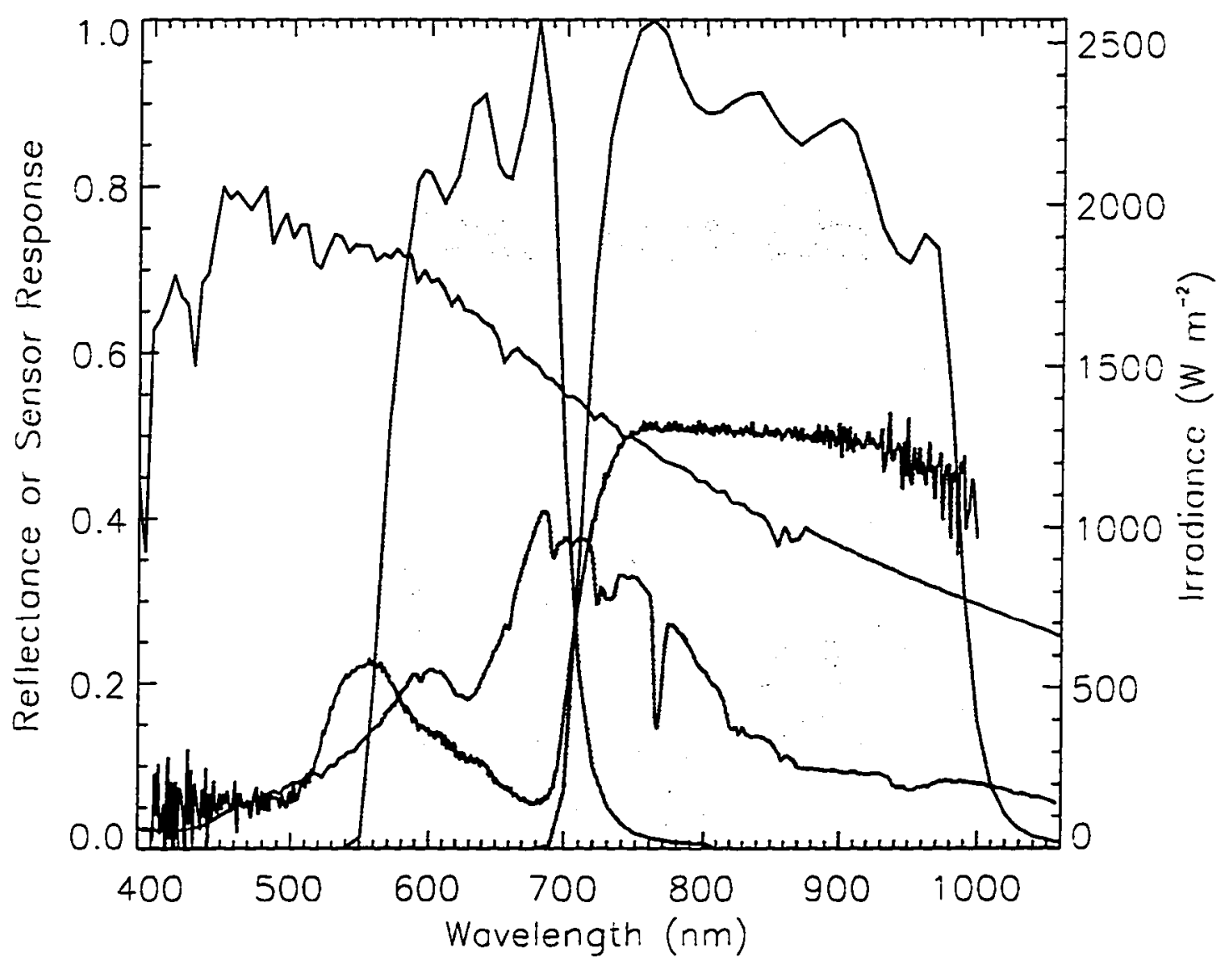

Figure 5.2 Typical leaf (green line) and soil (red line) reflectance spectra, the AVHRR visible and NIR sensor response curves (gray regions), and the solar spectrum (black line). 
properties of most terrestrial and oceanic surfaces. An exception would be associated with specular reflection (sun glint) of a smooth water body in the exact forwardscatter direction $\left(\theta=\theta^{\prime}\right.$ and $\psi=\pi$; see Fig. 5.1).

The BRF is sometimes inaccurately referred to as the bidirectional reflectance

distribution function (BRDF). The BRDF is a differential quantity equal to $d L_{0} / d E_{s}\left(s r^{-1}\right.$ ) (Wyatt 1978). While the BRDF theoretically describes the reflectance properties of a surface, it is not useful in practice, primarily because of the non-infinitesimal solid angle subtended by the source $E_{s}$. Thus the derivative above is not directly transformable into an actual remote sensing quantity.

Because of its usefulness for the types of large-scale problems we wish to address, data from the Advanced Very High Resolution Radiometer (AVHRR) will be central to the work in this chapter. The two wavelength bands in the optical region for this instrument are in the visible $\left(\Lambda_{1} \approx 0.58-0.68 \mu \mathrm{m}\right)$ and in the NIR $\left(\Lambda_{2} \approx 0.73-1.1 \mu \mathrm{m}\right)$. A more detailed picture of the AVHRR sensor response (bandwidth) is shown in Fig. 5.2. In the next section we will discuss the usefulness of the spectral dependence of the BRF and, in the following section (on inverse modeling), the combined spectral and geometrical (i.e., the directions of the vectors $\mathbf{r}$ and $\mathbf{r}$ ) dependence will be explored.

\subsection{Empirical Approaches: Vegetation Indices}

\subsubsection{Background}

A vegetation index (VI) is a scalar function of the discrete values $R_{1}$ of the BRF. The most common of these indices is the normalized difference vegetation index (NDVI). The NDVI is defined as a combination of visible (VIS) and near-infrared reflectance factors: 


$$
\mathrm{NDVI}=\frac{R_{\mathrm{NIR}}-R_{\mathrm{VIS}}}{R_{\mathrm{NIR}}+R_{\mathrm{VIS}}}
$$

and is most frequently used with the two-channel AVHRR optical data. Other important indices include the global environmental monitoring index (GEMI; Pinty and Verstraete 1992) and the soil adjusted vegetation index (SAVI; Huete 1988). These indices also are defined for use with visible and NIR wavelength bands, and have very similar properties as NDVI; the following discussion in this section is generally applicable to all three. The GEMI and SAVI are designed to reduce the effects of the atmosphere and soils, respectively, and contain adjustable parameters.

The fact that these indices are correlated with vegetation status (and not just structure) is owed to the unique spectral character of potentially active leaves. In turn, this spectral signanre is due primarily to the presence of chlorophyll A and B which absorb strongly in the blue and red wavelengths. Though there is generally a small peak in the green region, the primary leaf spectral feature that makes NDVI useful for terrestrial applications is the "red edge $^{n}$ : a sharp increase in reflectance and decrease in absorption from the red into the nearinfrared wavelengths that occurs at about $0.7 \mu \mathrm{m}$ (Fig. 5.2). The relatively high reflectance to the right of $0.7 \mu \mathrm{m}$ is a function of leaf optical thickness, controlled primarily by water content and mesophyll structure.

A number of ecological variables have been shown to be correlated with vegetation indices, particularly NDVI (e.g., Asrar et al. 1984, Running et al. 1989, Wiegand et al. 1991, Myneni et al. 1992b, Sellers et al. 1992). We briefly introduce some of these quantities below.

Leaf area index (LAI) is a dimensionless measure of the total leaf surface area per unit area of landscape. The terrestrial modeling community (in particular) has identified LAI as key 
variable in determining ecosystem-level exchange of water, energy, momentum and $\mathrm{CO}_{2}$ because it is a measure of both the physical extent of the canopy as well as its activity (i.e., photosynthesis/transpiration). LAI is also used as a measure of canopy depth, analogous to atmospheric pressure in meteorology. Light attenuation is an approximately exponential function of LAI. Thus, for heterogeneous landscapes or large areas, its meaning (an average LAI) is problematic for some applications.

Absorbed photosynthetically active radiation (APAR) is the absolute amount of PAR (photosynthetically active radiation) that is absorbed by the plant canopy. This quantity can be defined over multiple time scales, but generally it refers to either a daily integral value, or an instantaneous value. A related measure is the fraction of incident PAR that is absorbed (fAPAR). Canopy net photosynthesis $P_{c}\left(\mathrm{~g} \mathrm{~m}^{-2}\right)$ may be described by the following time-integral relationship (Asrar et al. 1984):

$$
P_{C}=\int_{T}\left[\varepsilon_{p} \cdot A_{e} \cdot f A P A R \cdot \mathrm{PAR}\right] d t
$$

where $\varepsilon_{p}$ is the photochemical conversion efficiency ( $\left(\mathrm{MJ}^{-1}\right.$ ), which is thought to be speciesspecific; and $A_{e}$ is a dimensionless environmental stress multiplier (accounting for light, water, or nutrient limitation), such that when $A_{e}=1, P_{c}$ is equal to potential net photosynthesis, or potential productivity. This relationship (which also has a theoretical basis) is the principal that underlies remote sensing of $f A P A R$.

Canopy photosynthetic efficiency is similar to fAPAR: it is the ratio of instantaneous canopy net photosynthesis to incident PAR, or $\left(\varepsilon_{p} \cdot A_{e} \cdot f A P A R\right)$. So, assuming constant environmental limitation and vegetation status over the period $T$, then it is equivalent to a constant times $f A P A R$. However, to the extent that $A_{e}$ changes over time, this quantity is not the 
same as $f A P A R$. Further, it has been suggested that correlations between plant growth and intercepted radiation are circular (Field 1991; see Section 5.4).

Stomatal resistance is best defined at the leaf level. $\mathrm{CO}_{2}$ (and water) exchange can be described as a Fick's Law process, obeying $F=\Delta c / R$; where $F$ is the gas flux, $\Delta c$ is the $\mathrm{CO}_{2}$ concentration gradient, and $R$ is the sum of aerodynamic resistance between the atmosphere and the leaf interior plus the resistance associated with the aperture of leaf pores (called stomata). Stomatal resistance is governed by the plant itself and represents a set of complex plant mechanisms which act to maximize carbon gain while minimizing water loss. It follows that the inverse, stomatal conductance. is linearly related to photosynthesis and transpiration. In the remote sensing literature. its canopy integral is considered. which is often just assumed to be inversely related to canopy photosynthesis and transpiration.

\subsubsection{Empirical basis for vegetation indices}

In practice, NDVI is usually related to macro-scale quantities like LAI, fractional absorbed (or intercepted) PAR, and fractional plant canopy cover using empirical relationships. Though there is a theoretical basis for most of these relationships (e.g., Sellers 1987), it is tarely invoked. Field measurements are typically used to build regressions which in turn are applied across the landscape, under the assumption that the relationships are uniformly applicable. To the extent that all the above applications work, they do so because they are correlated with one another. They all have a common basis: at the canopy scale, an increase in either of the above structural quantities (LAI, percent cover) is essentially an increase in the density of green leaves which absorb differentially in visible and NIR wavelengths, so there is a resulting increase in NDVI. Likewise. increased $f A P A R$ is the biophysical consequence of higher green leaf density. Of course, these dependencies arise because of the fundamental 
physico-chemical interactions at the leaf level, and variability in leaf optics will induce uncertainty in any of these interpretations. In fact, Myneni et al. (1995) present a unified theory that combines the three classes of dependencies, concluding that the broad-band vegetation indices are essentially an integrated measure of chlorophyll abundance. The principal factors that confound this interpretation are bidirectional effects (dependent on the angular distribution of leaves as well as the positions $\mathbf{r}$ and $\mathbf{r}^{\prime}$ ) and the reflecting properties of soil/background material.

Plant canopy reflectance (and thus NDVI) is sensitive to a number of ecosystem parameters. Privette et al. (1994) illustrated this sensitivity through the use of a canopy radiative transfer model that accounts for scattering processes using the discrete ordinates method. Sensitivity was expressed in terms of the differential change in reflectance per unit value of each parameter in the model (averaged over a number of base parameter configurations). They found that at low LAI (less that 1.5), visible reflectance was most sensitive to changes in LAI and soil reflectance to an equal degree, but NIR reflectance was most sensitive to soil reflectance, with LAI having secondary importance. At high LAI (greater than 2), the reflectance sensitivity was dominated by leaf reflectance for both wavelength bands, and in the NIR, leaf transmittance was almost equally important.

The implication for NDVI is intuitive: when LAI is low, either because the vegetation is sparse or because it is seasonal (deciduous), then variation in NDVI will result primarily from variation in soil color and LAI itself. In contrast, for relatively dense or closed-canopy ecosystems, variation in NDVI will result primarily from changes in leaf optics, either in the visible (because of changing chlorophyll concentration), or in the NIR (because of structural changes). Interestingly, however, many canopy radiative transfer models represent vegetation as a distributed "cloud" of leaves (turbid medium), so the effects due to non-photosynthetically 
active vegetation (NPV) are not included in the analysis just described. In practice, NPV components in the canopy, predominately stems, branches, and senescent foliage, are radiatively significant. The amount of NPV present in the canopy varies somewhat unpredictably in time and space, and particularly across ecosystem types. The model of Qin (1993) and the SAIL model as implemented by Braswell et al. (1996) both simulate a mixed canopy, so a full sensitivity could be performed with these models that includes the effect of NPV.

It is worthwhile to consider the qualitative meaning of NDVI in various ecosystems that have unique morphological and phenological characteristics. For example, in a typical shortgrass prairie, the NDVI will be highly seasonal, and the linear relationship with $f A P A R$ will be strongly affected by the mixture of live and standing dead vegetation, particularly near the beginning and end of the growing season. The relationship between NDVI and LAI will be less well-behaved; at the height of the growing season, changes in NDVI can be induced by water stress, which affects leaf optics as well as architecture (wilting). Interpreting NDVI in sparse communities (e.g., a shrubland) is the most problematic. Soil color and wetness will exert a strong influence over the NDVI signal. The seasonal amplitude will be very low, so LAI is practically impossible to determine accurately. In this case, it is likely that fractional vegetation cover is the dominant factor, and the most likely to be retrieved using NDVI. Similarly, in areas with mixed vegetation (i.e., partially evergreen) the observed reflectance will be strongly affected by a time-varying mixture of light scattered from soil. standing dead, or stems. Finally, in a deciduous forest, the interpretation for NDVI is similar to that of a seasonal grassland. The difference is that in the deciduous forest, soil/litter color influences are the strongest when LAI is low. The importance of leaf optical properties will dominate after 
canopy closure to a greater extent than in the short grassland by virtue of a (typically) greater LAI.

\subsubsection{Theoretical basis for vegetation indices}

Plant canopy radiative transfer theory is not singular; there are many models that range in complexity from semi-empiricism (Walthall et al. 1985) to full three dimensional calculations (Myneni et al. 1992a). Nevertheless, all the models (if they are correct) will reveal the same general relationships between NDVI and biophysical variables (e.g., LAI, biomass, component optical properties, and $f A P A R$ ) as are observed in the field. In general the more complex models contain fewer approximations, and are in principle more accurate, but only the most simple models are capable of producing closed-form analytic solutions. Models that are solved numerically are far more common.

The theoretical meaning of vegetation indices can be demonstrated in two ways: (1) through manipulation of equations that coarsely describe light interaction in plant canopies; or (2) by integration of a numerical canopy radiative transfer model and inspection of the results for various parameter combinations. In the following sections we will give a brief overview of one analytical argument from the literature, and finally, we will present some SAIL model results that show how the relationships may depend on location-specific factors.

\section{Analytical relationships}

Sellers (1987) presented one of the few available analytical foundations for the relationship between VIs and canopy variables. They based their discussion on the two-stream approximation (Ross 1975. Dickinson 1983) which describe canopy light interaction in terms of two fluxes: downwelling diffuse radiation $\left(E_{-}\right)$and upwelling diffuse radiation $\left(E_{+}\right)$. Further, it 
is assumed that these fluxes exist in a plane parallel medium that is composed of isotropic scattering elements distributed uniformly in horizontal space. The scattering elements (leaves) are angularly distributed in an arbitrary manner according to $G(\mu)$, where $\mu=\cos (\theta)$. In the two-stream approximation, the divergence of scattered radiation within the canopy is given by:

$$
\begin{aligned}
& \bar{\mu} \frac{d E_{+}}{d L}+[1-(1-\beta) \omega] E_{+}-\omega \beta E_{-}=\omega \bar{\mu} K \beta_{0} \mathrm{e}^{-K L} \\
& \bar{\mu} \frac{d E_{-}}{d L}+[1-(1-\beta) \omega] E_{-}-\omega \beta E_{+}=\omega \bar{\mu} K\left(1-\beta_{0}\right) \mathrm{e}^{-K L}
\end{aligned}
$$

where,

$$
\begin{aligned}
& \mathrm{K}=G(\mu) / \mu, \text { the source attenuation coefficient or optical depth per unit LAI, } \\
& \mathrm{L}=\text { the cumulative leaf area index (LAI), } \\
& \bar{\mu}=\int_{0}^{1}\left[\mu^{\prime} / G\left(\mu^{\prime}\right)\right] d \mu^{\prime}=(\overline{1 / h}) \text {, the average inverse diffuse optical depth per LAI, } \\
& \omega=\alpha+\tau \text {, reflectance plus transmittance, the scattering coefficient, } \\
& \beta, \beta_{0}=\text { upscatter parameters for diffuse and direct beams, respectively. } \\
& \mu=\cos (\theta) \text {, where } \theta \text { is the direction of incident solar radiation. }
\end{aligned}
$$

Note that $\omega$ and $\beta$ depend on wavelength, so two sets of equations are applicable when considering NDVI (one for visible and the other for near-infrared wavelengths). Equation 5.8 is a special case of the diffuse turbid medium formalism (Equation A.3), where the scattering coefficient $\sigma$ is equal to $(\omega \beta / \bar{\mu})$ and the absorption coefficient $\kappa$ is equal to $((1-\omega \beta) / \bar{\mu})$. Both these coefficients from (A.3) are weighted by $1 / \bar{\mu}$ to allow for a generalized leaf angle 
distribution, however, $\vec{\mu}$ is equal to unity for the case of spherically or horizontally distributed leaves, and is approximately equal to unity for other distributions. Though the definition of $\bar{\mu}$ is "somewhat arbitrary" (Dickinson. 1983) it can be derived directly from the plane-parallel radiative transfer equation (Choudury 1987); it requires, however, the approximation that $E_{+}$ and $E_{\text {. are isotropic. }}$

The terms in Equation 5.8 have a physical interpretation: $(1-(1-\beta) \omega)$ is the amount of radiation transmitted by a single element $d L$ in the medium, $\omega \beta$ is the reflected radiation, and an exponentially declining source term is on the right hand side. The factor $\beta$ is a function of the mean leaf inclination angle and accounts for the overlap between the upper (lower) hemisphere of the leaf and the upper (lower) hemisphere relative to the local normal (Ross 1975). The solution to (5.8) can be obtained using standard methods, and under appropriate boundary conditions, is a lengthy expression containing terms proportional to $\exp (-K L)$ and $\exp (-h L)$, where $h$, the diffuse attenuation coefficient, is a function of the leaf scattering coefficient $\omega$.

Canopy hemispherical (as opposed to bidirectional) reflectance, or albedo, is then given by $a(\mu)=E_{+}(0)$. Now the simple ratio (SR) spectral vegetation index can be defined:

$$
\mathrm{SR}=\frac{a_{\mathrm{NIR}}}{a_{\mathrm{VIS}}}
$$

The quantity SR behaves very much like NDVI over the possible values of NDVI (they are nearly proportional, which can be shown by expanding both quantities in a Taylor series). Further, the SR is much easier to deal with analytically, so conclusions can be made using SR and then extended to NDVI. 
In order to investigate the relationships between SR and biophysical variables, the approach is to evaluate the derivative of (5.9) with respect to total LAI and then compare to the representation of APAR, canopy photosynthesis, and canopy resistance implied by the solution to (5.8). The resulting equations are worked out in detail by Sellers (1987), and we will outline the results below. Inclusion of canopy physiology is by way of theory relating photosynthesis and transpiration to light intensity and other environmental variables introduced by Farquar and Sharkey (1982), which essentially describes the exponential decrease in photosynthesis per unit leaf area as a function of LAI. Another useful relationship is the expression for absorbed PAR:

$$
\operatorname{APAR}=1-a_{V}-\left(1-\rho_{s}\right)\left[\mathrm{e}^{-K_{v} L_{\tau}}+I_{d}\left(L_{\tau}\right)\right]
$$

where $L_{T}$ is the total canopy LAI, which assumes that PAR wavelengths are equivalent to visible wavelengths.

A few simplifications are introduced by Sellers (1987). First, it is assumed that the derivative of $a_{V}$ with respect to $L_{T}$ is much smaller than the derivative of $a_{N}$ with respect to $L_{T}$, and that $a_{V}$ itself is much smaller than $a_{N}$. Both of these assumptions are generally supported by laboratory and field measurements. Further, it is assumed that soil reflectance in the visible wavelengths is approximately equal to leaf reflectance in the same region. This is often true, particularly over dark soils, but does not hold in general. The following statements of proportionality can then be obtained:

$$
\frac{\partial(S R)}{\partial L_{T}}, \frac{\partial\left(a_{N}\right)}{\partial L_{T}} \propto \mathrm{e}^{-2 h_{s} L_{\tau}}
$$




$$
\frac{\partial(A P A R)}{\partial L_{\tau}}, \frac{\partial\left(P_{C}\right)}{\partial L_{T}}, \frac{\partial\left(1 / r_{C}\right)}{\partial L_{\tau}} \propto \mathrm{e}^{-K_{v} L_{\tau}}
$$

where $h_{N}$ is the extinction coefficient for diffuse near infrared light, and $K_{V}$ is the extinction coefficient for incident visible light. The left-hand terms are all linearly related to one another and all exhibit exponentially decreasing sensitivity to LAI.

Finally, if $2 h_{N} \approx K_{V}$, then the terms on the left side of (5.11) and (5.12) will be proportional to one another. This last assumption that the optical depth in a canopy is twice as large for NIR than visible solar radiation, which is not intuitive, also turns out to be approximately true (over a wide variety of canopy architectures) because of the differential absorptance of leaves. but it depends on the exact meaning of "visible" and "near-infrared". Remote sensing instruments differ in spectral band widths, and Sellers (1987) notes that in order to best infer APAR or the other quantities from NDVI or SR, then the sensor bands (that are associated with $a_{V}$ and $a_{N}$ ) should be chosen such that last assumption is as generally applicable as possible.

\section{Model results}

The requirement of many simplifying assumptions in above analytical discussion reveals the advantage of using a numerical model to investigate the functional relationships between VIs and the biophysical/ecological parameters of interest. Though the functions themselves may differ with varying model complexity, in general the functional forms are robust from the simplest representations to the most complex. We present a few brief examples using a canopy radiative model that is intermediate in complexity: the SAIL (Scattering by Arbitrarily Inclined Leaves) model (Verhoef 1984). which uses the following parameter set: 
LAI, leaf reflectance, leaf transmittance, soil/litter reflectance, and mean leaf inclination angle (assuming an elliptical distribution). The version used by Braswell et al. (1996) also includes non-photosynthetic vegetation (NPV) scattering elements, a hot-spot parameterization for both leaves and NPV, and variable fractional vegetation canopy cover (for details see Section 5.3 and Appendix A).

For a typical parameter set, Fig. 5.3a shows the visible and NIR reflectance predicted by SAIL as a function of LAI. In the lower panel (Fig. 5.3b), the NDVI and $f A P A R$ for the same model canopy are shown. This figure illustrates the most conceptual explanation of the powerful and near-linear relationship between NDVI and fAPAR: (1) light attenuates exponentially with depth in a plant canopy because leaves absorb more radiation than they reflect, so as LAI increases, $f A P A R$ increases, but the differential change in the canopy light environment decreases; (2) leaves absorb much more in the visible than in the NIR ( $90 \%$ vs. $-20 \%$ ), so NDVI increases with LAI, but because of the saturating effect at higher LAI values, both NDVI and fAPAR approach an asymptotic value. Given that NDVI and fAPAR are approximately described by $A\left(1-\mathrm{e}^{-k L}\right)$ and that $A$ and $k$ for both quantities are nearly equal, then they are approximately linearly correlated. We present this plausibility argument as a complement to the theoretical presentation of Sellers (1987) and to the wealth of experimental evidence for such relationships.

Figure 5.4 shows the results of two other SAIL model runs. The parameter set used was derived from a model inversion at two locations along a transect in central Africa (see Braswell et al. 1996; Section 5.3): one near the northern end (dashed line), dominated by grasses; and one near the southern end (solid line), dominated by closed-canopy forest. The exponential decrease in the sensitivity of both NDVI and fAPAR can be seen clearly, with some differences due to the optical and (primarily) the structural differences in the two canopy types. 

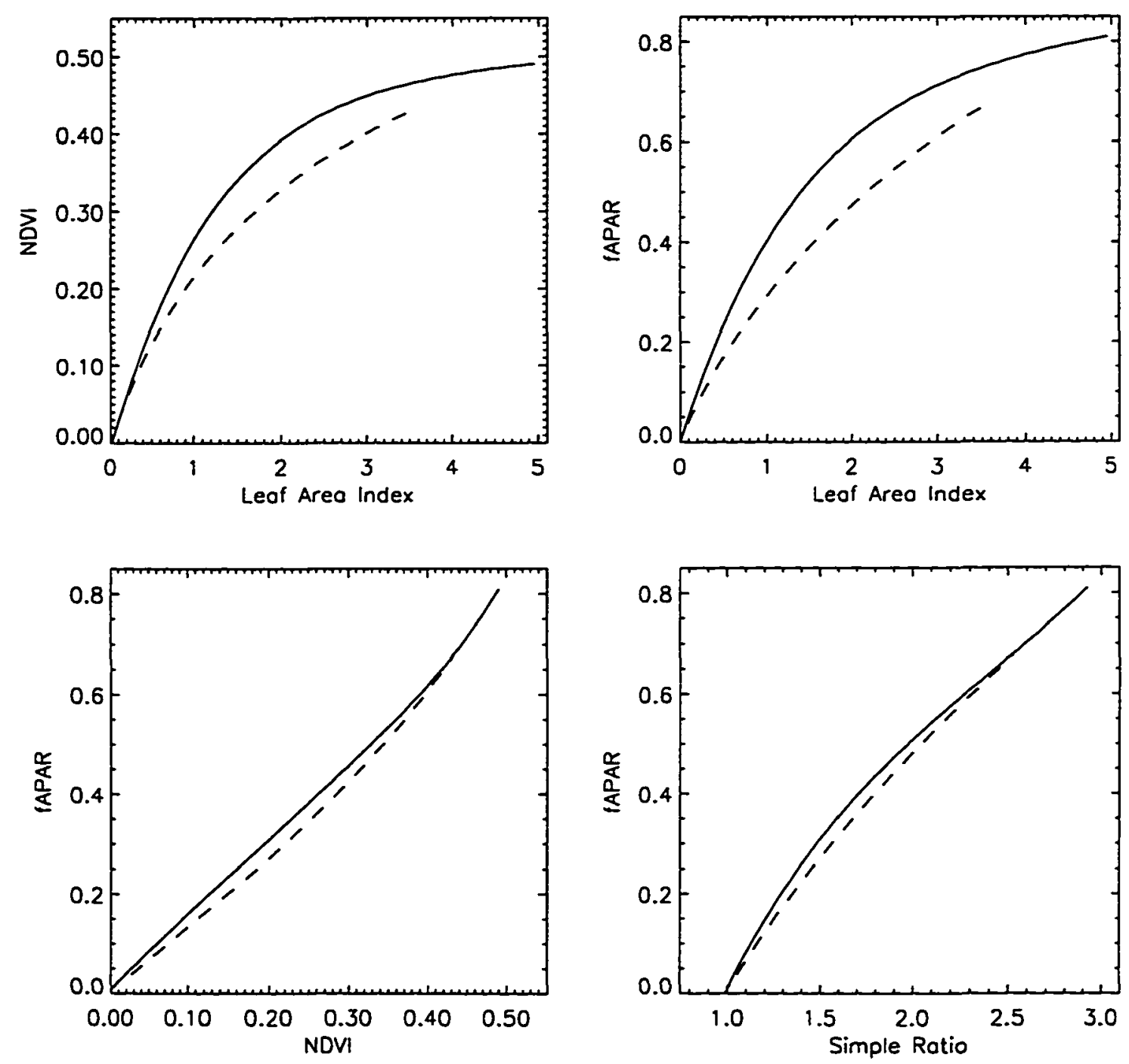

Figure 5.3 (a) Visible (solid line) and NIR (dashed line) reflectance simulated by the SAIL canopy radiative transfer model as a function of leaf area index. (b) Fractional PAR absorbed (solid line) and NDVI (dashed line) for the same model canopy as in (a). Typical parameter values are: Leaf vis. reflectance $=$ leaf vis. transmittance $=0.1$, Leaf NIR reflectance $=$ leaf NIR transmittance $=0.3$, Soil vis. reflectance $=$ Soil NIR reflectance $=0.2$, Stem area index $=1.0$, Stem vis. reflectance $=$ stem NIR reflectance $=0.2$, Stem vis. transmittance $=$ stem NIR transmittance $=0.0$, Mean leaf inclination $=45^{\circ}$, Mean stem inclination $=60^{\circ}$, Leaf hot spot parameter $=0.2$, Stem hot spot parameter $=0.5$. Fractional vegetation cover $=0.8$, horizontal visibility $=50 \mathrm{~km}$. 

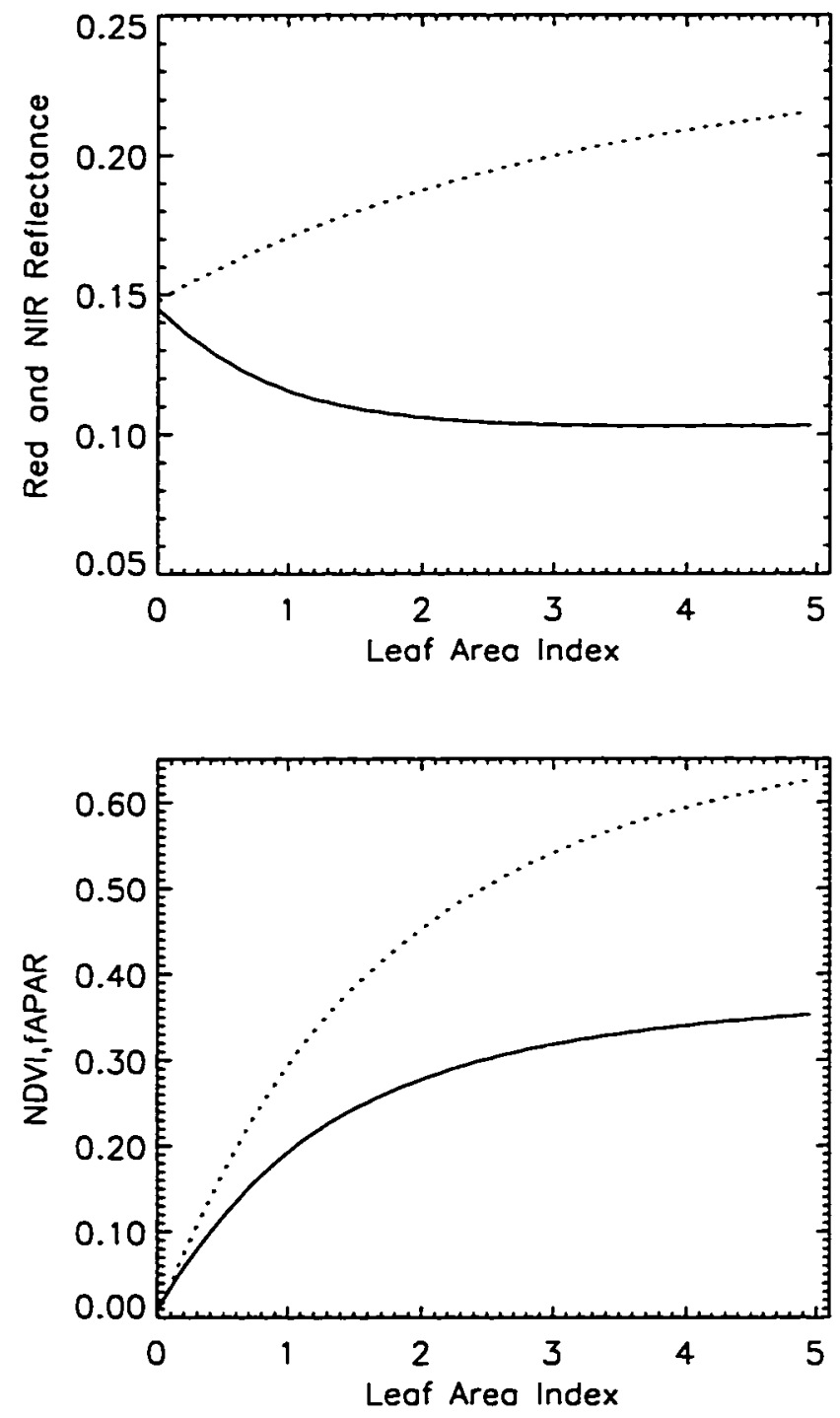

Figure 5.4 Results from two SAIL model simulations. Solid lines corresponds to estimated parameters for evergreen forest, and dashed lines correspond to estimated parameters for a grassland-savanna mixture. 
The NDVI and SR relationships, however are remarkably stable with respect to those parameters, which is consistent with the idea that NDVI is fundamentally related to chlorophyll density (Tucker 1979, Myneni et al. 1995). It is important to note, however, that we have plotted values for the green-leaf LAI and $f A P A R$ only. Field and remote sensing measurements will contain an effect of absorption of visible light by NPV, which can be large. The bottom panels of Fig. 5.4 do not necessarily support the blind use of NDVI for fAPAR for two other reasons: (1) plotting daily integrated $f A P A R$ (the quantity of interest with respect to canopy photosynthesis calculations) would cause the lines to diverge further because of solar zenith angle effects; and (2) the two sites have similar retrieved soil and leaf optical properties which were estimated through inversion and which would in general need to be accounted for in order to exploit the linear relationship.

\section{Summary}

The use of remotely-sensed vegetation indices as a direct proxy for surface variables ignores some major dependencies, even when atmospheric corrections have been applied to the reflectance data. Predominately, these are the effects of solar and viewing geometry and the effects of variable background reflectance. Moreover, because of its asymptotic dependence, NDVI can be used to infer LAI only over a limited range of LAI. Although the relationship between NDVI and $f A P A R$ is generally linear, it is well-known to be sensitive to the status of many soil-vegetation-atmosphere system parameters that exhibit high spatial and/or temporal variability (Goward and Huemmrich 1992). Thus, a one-to-one relationship between a VI and a biophysical variable may not be generally applicable, except through careful consideration of complicating factors; by inventing indices that are less sensitive to atmospheric or soil influences (Huete 1988, Pinty and Verstraete 1992), by normalizing the index (Roujean and 
Breon 1995), or by selecting pixels with preferred view angles (Cihlar et al. 1994). Such considerations (especially the latter two) potentially involve the iterative use of radiative transfer (RT) models which is similar, in principle, to the method of inversion.

\subsection{Extracting Ecological and Biophysical Information from AVHRR Optical Data: An Integrated Algorithm Based on Inverse Modeling ${ }^{\dagger}$}

\subsubsection{Introduction}

To obtain information about vegetation structure and function from remote sensing, a model is needed to convert the signal measured by the satellite sensor (reflected solar radiation) into variables that are meaningful at the stand or ecosystem level. The inverse modeling approach uses physical models of the radiation regime within a plant canopy to retrieve information about the surface. Specifically, models are required that simulate the plant canopy reflectance given a unique parameterization of the structural and optical characteristics of the vegetated surface. In this approach, an optimization scheme is used to calculate the parameter set that yields modeled reflectance most consistent with the observed reflectance data.

There are a number of distinct advantages to radiative transfer model inversion: it is physically-based so that the relationship between measured reflectance and surface biophysics is self-adjusting instead of empirically calibrated, it deals with mechanisms that would otherwise contaminate the signal, and it allows the incorporation of ecological knowledge and field measurements in a consistent way. The degree to which the model physics (assuming they are adequately represented) adjust to novel environments depends on the quality of the ancillary ecological information provided, but the retrieved parameters should not be less accurate those derived from methods that do not incorporate such data. The main disadvantage of inversion is

\footnotetext{
${ }^{\dagger}$ Adapted from the article by B.H. Braswell, D.S. Schimel, J.L. Privette, B. Moore III, W.J. Emery, E.W. Sulzman, and A.T. Hudak, Journal of Geophysical Research (Almospheres) 101:23335-23348. 1996. Copyright by the American Geophysical Union.
} 
that it is computationally more intensive. The requirement of multiple measurements may be considered a disadvantage but, in this section, we will demonstrate a practical solution to that problem.

The objectives of this section are (1) to present an integrated algorithm for the retrieval of fractional APAR ( $f A P A R)$, albedo, and phenology from AVHRR reflectance measurements by inverting a modified version of the SAIL canopy radiative transfer model, and (2) to present successful inversion results that demonstrate the potential for this algorithm to be used operationally. Past radiative transfer model inversions have been limited to point analyses. We extend these efforts by developing a technique that facilitates inversions at regional to global scales, and that explicitly incorporates a priori knowledge in the choice of parameter values and constraints. Our method works with the AVHRR Pathfinder product, which is readily available, and the only current global reflectance data set suitable for this use.

\subsubsection{The AVHRR Pathfinder data product}

Since 1981, optical reflectance data has been gathered from the AVHRR on board the NOAA-7 through 11 polar orbiting platforms. This instrument measures exiting radiance in five channels: red (VIS; 0.58-0.68 $\mu \mathrm{m}$ ), near-infrared (NIR; 0.73-1.10 $\mu \mathrm{m}$ ), and three thermal infrared bands. The satellite approximately covers the globe each day, and the highest spatial resolution possible is about $1.1 \times 1.1 \mathrm{~km}$. The Pathfinder AVHRR Land (PAL) product (Agbu and James 1995) is a global data set, with $8 \times 8 \mathrm{~km}$ spatial resolution and a 10-day compositing interval. The data are projected onto a Goode's Homolosine equal-area map base. There are twelve data layers; each pixel contains the two optical reflectances $\left(R_{\text {VIS }}^{*}\right.$ and $R_{\text {NIR }}^{*}$, temperatures from the three thermal bands, sun and sensor zenith angles $(\theta$ and $\theta$ ), the relative azimuth angle $(\psi)$, and the day/hour of observation. Also included is a quality control (QC) 
flag, an estimate of the extent of cloud contamination calculated using the thermal bands, and NDVI. The QC and cloud flag layers are described in detail in Agbu and James (1995) and are used in our algorithm to filter pixels that potentially contain errors or extensive cloud contamination.

The Pathfinder reflectances are derived from the "global area coverage" (GAC) data that are produced on board the satellite. Because of on-board data storage limitations, the nominal $1.1 \mathrm{~km}$ "local area coverage" (LAC) spatial resolution of AVHRR is not automatically retained for the entire globe. Instead, measurements are aggregated by averaging four adjacent samples out of every five in a scan line. then skipping the next two scan lines (Kidwell 1991). The resulting GAC spatial resolution is approximately $1 \times 4 \mathrm{~km}$ at nadir. In the Pathfinder data set, pixels are mapped onto a global grid from the raw GAC data. Global area coverage data has coarser resolution and potentially poorer quality than the $1.1 \mathrm{~km} \mathrm{LAC}$ data because of reduced spatial sampling, but logistical issues associated with recovering LAC measurements are being addressed by an IGBP core project (Townshend et al. 1994) and global "1 km AVHRR" data will soon be available for a limited time domain, including the ancillary information (described above) that is necessary for our analysis.

The data are temporally composited (during Pathfinder processing) by choosing the GAC reflectances and the associated ancillary information from the observation that yielded the highest NDVI in the 10-day window, a common practice because almost all factors (particularly aerosols) that contaminate the signal from the land surface tend to reduce NDVI (Holben 1986). Bidirectional effects on NDVI are a complex function of sun-sensor geometry and land surface conditions and thus they will not be selected out of the data by this compositing technique. Moreover, the compositing process has the effect of increasing the sampling of sun-sensor geometry from pixel to pixel because the uncorrected atmospheric 
effects that lead to decreased NDVI tend to be spatially and temporally heterogeneous, and because each consecutive overpass will have a unique (random) scan angle. It is therefore very likely that nearby pixels in the final product are derived from different overpasses. This effect of compositing on the local distribution of sun-sensor angles facilitates the application of the method introduced in this paper.

The PAL global data product currently covers 1981-1994 (see WWW site http://xtreme.gsfc.nasa.gov). In our analysis we have used two subsets of the data for two related applications. First, an initial large-scale stratification and geostatistical description was performed using all of the 10-day composited data for the African continent for the years 19861988. Second, for the set of inversion experiments, we extracted clusters of pixels (cells) along a transect in central Africa $\left(19.7^{\circ} \mathrm{E}, 8.1^{\circ} \mathrm{N}\right.$ to $\left.22.3^{\circ} \mathrm{E}, 4.3^{\circ} \mathrm{N}\right)$ for all twelve months of 1986.

\subsubsection{Theoretical background}

Spaceborne radiometers (e.g., AVHRR) measure solar radiation that has been transmitted through the atmosphere, interacted with the surface (including vegetation, soil, water, etc.), and then transmitted back through the atmosphere to the satellite. A radiometer measures the photon energy flux received within fixed intervals of wavelength and solid angle. This information can be translated into reflectance, a physical variable that is independent of instrument characteristics and solar flux, through radiometric calibration (Brown et al. 1985). The variation of reflectance with respect to wavelength and sun-sensor geometry is called the bidirectional reflectance factor (BRF) and is given by

$$
R\left(\mathbf{r}, \mathbf{r}^{\prime}, \lambda\right)=\frac{\pi L(\mathbf{r}, \lambda)}{E_{s}\left(\mathbf{r}^{\prime}, \lambda\right)}
$$


where $E_{S}$ is the solar irradiance $\left(\mathrm{W} \mathrm{m}^{-2}\right.$ ) incident from the $\mathbf{r}$ direction, and $L$ is the outgoing radiance $\left(\mathrm{W} \mathrm{m}^{-2} \mathrm{sr}^{-1}\right)$ measured at $\mathbf{r}$ (see Section 5.1 ). This is an approximate expression because there is usually a small flux of radiation incident from all directions ("skylight") in addition to the direct solar beam.

Canopy radiative transfer models simulate the BRF for a given wavelength band as a function of sun-sensor geometry, and a set of parameters $\mathbf{P}$ describing the architectural and optical characteristics of the components for the vegetated surface, i.e., $R=R_{\mathbf{P}}(\mathbf{r}, \mathbf{r}, \lambda)$. In this study, we used the SAIL model (Verhoef 1984,1985 ) because it is intermediate in complexity, and represents a compromise between physical realism and the number of input parameters. Parameters of the SAIL model include leaf area index (LAI), leaf optical properties, and leaf angle distribution. The SAIL model calculates $R_{\mathbf{P}}$ by solving the radiative transfer equations for scattering of (diffuse and direct) sunlight by a distribution of small flat elements (leaves) in a semi-infinite medium, while allowing for a linear mixing of bare soil reflectance.

We have modified SAIL to simulate the reflectance of two-component canopies (cf., Qin 1993), including a hot-spot parameterization (Kuusk 1991) for both components. Table 5.1 shows the full set of parameters used in this implementation of the model (SAIL-2). It is important to represent non-photosynthetic vegetation (NPV) components in a radiative transfer model because almost all plant canopies contain stems, standing dead, or senescent leaves that interact with radiation and affect estimates of physical parameters. For example, fully senescent grasslands in the dry season can absorb a significant amount of radiation in the PAR wavelengths that is not associated with biological activity. The hot-spot parameterization allows 
Table 5.1 The SAlL-2 model parameters, and their bounds or default values for 3 inversion experiments.

\begin{tabular}{|c|c|c|c|c|c|c|c|}
\hline \multirow[b]{2}{*}{$i$} & \multirow[b]{2}{*}{ Parameter $\left(P_{i}\right)$} & \multicolumn{2}{|c|}{ Configuration $\mathrm{Gl}$} & \multicolumn{2}{|c|}{ Configuration G2 } & \multicolumn{2}{|c|}{ Configuration $\mathrm{F}$} \\
\hline & & Mode & Value & Mode & Value & Mode & Value \\
\hline 1 & Plant area index & free & $0.5-10.0$ & free & $0.5-10.0$ & free & $0.5-10.0$ \\
\hline 2 & Stem fraction & free & $0.0-0.5$ & free & $0.5-1.0$ & free & $0.1-0.9$ \\
\hline 3 & Leaf red reflectance & range & $0.05-0.17$ & free & $0.05-0.17$ & free & $0.05-0.17$ \\
\hline 4 & Leaf NIR reflectance & linked & $f\left(P_{3}\right)$ & linked & $f\left(P_{3}\right)$ & linked & $f\left(P_{3}\right)$ \\
\hline 5 & Leaf Red transmittance & linked & $f\left(P_{3}\right)$ & linked & $f\left(P_{3}\right)$ & linked & $f\left(P_{3}\right)$ \\
\hline 6 & Leaf NIR transmittance & linked & $f\left(P_{3}\right)$ & linked & $f\left(P_{3}\right)$ & linked & $f\left(P_{3}\right)$ \\
\hline 7 & Stem red reflectance & free & $0.1-0.4$ & range & $0.1-0.4$ & range & $0.1-0.4$ \\
\hline 8 & Stem NIR reflectance & linked & $f\left(P_{7}\right)$ & linked & $f\left(P_{7}\right)$ & linked & $f\left(P_{7}\right)$ \\
\hline 9 & Mean leaf angle & linked & $f\left(P_{7}\right)$ & linked & $f\left(P_{7}\right)$ & fixed & 0.0 \\
\hline 10 & Stem NIR transmittance & linked & $f\left(P_{7}\right)$ & linked & $f\left(P_{7}\right)$ & fixed & 0.0 \\
\hline 11 & Mean leaf angle & fixed & $50^{\prime \prime}$ & fixed & $50^{\circ}$ & fixed & $50^{\circ}$ \\
\hline 12 & Mean stem angle & fixed & $50^{\prime \prime}$ & fixed & $50^{\circ}$ & fixed & $60^{\circ}$ \\
\hline 13 & Leaf hot spot parameter & fixed & 0.4 & fixed & 0.4 & fixed & 0.1 \\
\hline 14 & Stem hot spot parameter & fixed & 0.4 & fixed & 0.4 & fixed & 0.5 \\
\hline 15 & Soil red reflectance & range & $0.06-0.4$ & range & $0.06-0.4$ & range & $0.06-0.4$ \\
\hline 16 & Soil NIR reflectance & linked & $f\left(P_{15}\right)$ & linked & $f\left(P_{15}\right)$ & linked & $f\left(P_{15}\right)$ \\
\hline 17 & Horizontal visibility & fixed & $50 \mathrm{~km}$ & fixed & $50 \mathrm{~km}$ & fixed & $50 \mathrm{~km}$ \\
\hline 18 & Cover fraction & range & $0.5-0.8$ & range & $0.8-1.0$ & fixed & 0.99 \\
\hline
\end{tabular}

The mode indicates how each parameter was treated in the inversion process: "free" means optimization was performed to retrieve these parameters, "fixed" means these parameters were specified prior to inversion and held constant, "range" means these parameters were fixed for each optimization in an ensemble, and "linked" mean these parameters were determined as a function of some other parameter (based on field data). 
for the treatment of self-shading effects of both components: when the observer is looking from the back-scatter direction, fewer shadows are seen than from the forward-scatter direction, leading to greater observed radiance (a reflectance peak at $\mathbf{r}=\mathbf{r}^{\prime}$ ). The width of this peak is related to the shape and size of canopy elements, thus the hot-spot parameter is representative of the ratio of leaf (or stem) size to canopy height and varies from 0 to 1 (Kuusk 1991).

The most common method of inverting a radiative transfer model is to use an iterative optimization procedure that attempts to find the global minimum of a least-squares measure of error, the merit function:

$$
E^{2}(\mathrm{P})=\sum_{i} \sum_{j} w_{i j} \cdot\left[R^{*}\left(\Theta_{i}, \lambda_{j}\right)-R_{\mathrm{P}}\left(\Theta_{i}, \lambda_{j}\right)\right]^{2}
$$

where $E^{2}$ is the sum-squared error, $\Theta_{i}=\left(\theta_{i} \theta_{i}^{\prime} \Psi_{i}\right)$ represents the set of sun-sensor geometries $\left(\mathbf{r}_{i} \dot{\mathbf{r}}_{i}\right), R^{*}$ is the measured reflectance, and the summation is over the total number of measurements at hand: the unique geometrical configurations $(i=1, \ldots, N)$ times the number of spectral bands per measurement. Goel and Thompson (1984) have shown that the SAIL model is mathematically invertable in this manner. In the case of AVHRR, $j$ is either 1 (visible) or 2 (near-infrared). In addition, a set of weighting coefficients $w_{i j}$ may be applied to reflect the relative importance of directions and wavelengths with respect to the slope of the merit function and thus improve the convergence of the optimization. We have left all $w_{i j}=1$ in this study, but the usefulness of non-unit weights has been demonstrated by Privette et al. (In press). Equation (5.14) assumes that there is a multiple sampling of geometry, which is not strictly possible with AVHRR for a single target and satellite overpass, but we present one method for approximating this sampling in the next section. 
Given that the principal independent variables are $\Theta$ and $\lambda$, the "shape" of the BRF with respect to the sun-sensor orientation and to wavelength is the fundamental profile used to characterize the land surface. A basic fact of remote sensing is that in order to retrieve some quantity $P_{i}$, the BRF must be sensitive to small changes in that parameter, i.e.,

$$
\frac{d R(\Theta, \lambda ; \mathrm{P})}{d P_{i}}>\varepsilon
$$

where $\varepsilon$ represents some measure of the noise threshold in the data. As an example, reflectance in both the red and NIR bands saturates with increasing LAI. This implies (as mentioned earlier) that the derivative in (5.15) approaches zero, and thus it is increasingly difficult to determine LAI at high values of LAI. An additional directional measurement (i.e., incremental sampling of $\Theta$ ) is useful for determining $\mathbf{P}$ when the derivative with respect to some parameter $P_{i}$ at the new measurement geometry $\Theta^{\circ}$ is not equal to the derivative at any other available $\Theta_{i}$. The measurement is then said to be independent (or unique) and it contains new information. The number of independent measurements needed depends on the quality of model and data, the nature of the target, and the desired number of retrieved parameters.

If there are $M$ parameters and $2 N$ measurements, and if one attempts to retrieve $m<M$ free parameters, then an obvious requirement is that $2 N \geq m$. However, due to the existence of noise in the data and inaccuracy in the model it is desirable to have $N$ as large as possible and $\mathrm{m}$ as small as possible. Because reducing the number of free parameters $m$ in the inversion increases the likelihood of a successful optimization (retrieval), those with less sensitivity can be held fixed at little cost to accuracy (Privette et al. In press). In addition, if functional relationships can be found between parameters, it will reduce the dimensionality of the 
parameter space. Finally, it may also be useful to apply an ensemble of fixed parameters and average the resulting retrieved parameters. All of these techniques have been used in this study.

When an acceptable parameter set $\mathbf{P}$ of the $\mathrm{RT}$ model has been recovered from the data, i.e., a minimum of $E^{2}(\mathbf{P})$ has been found, daily total $f A P A R(\Pi)$ (e.g., Goward and Huemmrich 1992) is obtained by diurnal integration:

$$
\Pi=\frac{\int_{T} d t\left[\cos \left(\theta^{\prime}(t)\right) \cdot f_{\mathrm{P}}\left(r^{\prime}(t), \lambda_{s}\right) \cdot E_{s}(\lambda)\right]}{\int_{T} d t\left[\cos \left(\theta^{\prime}(t)\right) \cdot E_{s}(\lambda)\right]},
$$

where $T$ is the number of daylight hours (dependent on latitude and time of year), $\lambda_{\pi}$ is the photosynthetically active radiation (PAR) portion of the spectrum, and $f_{\mathrm{P}}$ is the instantaneous fractional PAR absorbed by the canopy. Albedo $(\alpha)$ is computed by hemispheric integration (Ross 1981):

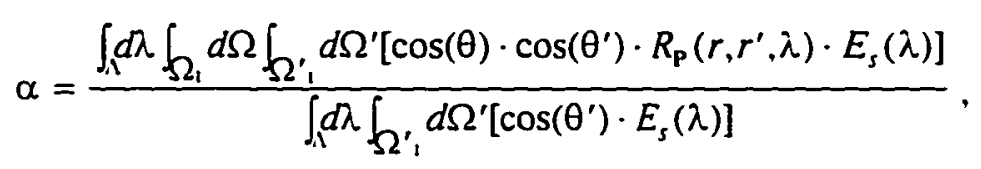

where $\Lambda$ is the entire solar spectrum $(-0.03-1.2 \mu \mathrm{m})$ and $\Omega_{1}$ is the upper hemisphere.

\section{$\underline{5.3 .4 \text { Methods }}$}

The basis of our approach is the simulation of a geometrical sampling of the BRF by aggregating the reflectance data into cells that contain many individual $8 \mathrm{~km}$ pixels, retaining the associated location-specific values of reflectance, sun-sensor geometry, and quality-control indicators. Because of the Pathfinder compositing, spatially-adjacent pixels are often obtained 
with significantly different sun-sensor angles. Thus, within regions and 10-day composites, significant angular information exists, although each pixel is at a single angle. We reasoned that most relatively undisturbed systems, which are heterogeneous at sub-km scales, may represent reasonably homogeneous mixtures at $1-\mathrm{km}$ scales and larger, and that pixels within a region may each contain consistent mixtures of the same types (e.g., $x \%$ trees, $y \%$ shrubs and $z \%$ grasses). Thus, multiple pixels within some grid cell could be used to produce a synthetic BRF for that grid cell, simulating multiple measurements of a (cell-sized) target. Appendix B contains supplementary information on most of the methods presented in this section.

\section{Stratification of the Continental-Scale Data Using a Vegetation Index}

We chose a region in central Africa for initial testing. The region was chosen because it is the site of an ongoing interdisciplinary investigation and because it contains extensive continuous mixtures of natural vegetation. The first step was to perform a series of large-scale analyses with AVHRR data using a vegetation index in order to assess: (1) the appropriate cell size and shape, and (2) a basis for excluding pixels from a cell that are outliers. We took NDVI from the Pathfinder data for the African continent and created a VI climatology by recompositing to maximum monthly NDVI, cloud and QC filtering, then averaging over the three years for each month, i.e.,

$$
\left.\overline{\mathrm{NDVI}}_{i}=\frac{1}{3}\left[\sum_{j} \text { filt(max }\left(\mathrm{NDVI}_{i j}\right)\right)\right], \quad i=(\mathrm{Jan} . . . \mathrm{Dec}) ; j=(1986 \ldots 1988)
$$

We then performed a principal components $(\mathrm{PC})$ rotation on the $\overline{\mathrm{NDVI}}_{i}$ to define a set of new, orthogonal variables. The first three PCs explain 96 percent of the total variance. As the 
principal components of a stationary time series are equivalent to Fourier components, the first three PCs roughly correspond to the mean greenness, and the amplitude and phase of the seasonal cycle. Higher-order components are significant only for some isolated areas, amounting to a very small percentage of all pixels in Africa. The images in Fig. 5.5a,b show the first three PCs as a mixture of red(1), green(2), and blue(3). Functional ecotypes can be identified as having approximately the same color; this mapping may be thought of as a continuous, unlabeled vegetation characterization. A discrete characterization can also be obtained by clustering the PCs into a number of unique, idealized classes. Similar multitemporal characterizations of spatial data have been presented in the literature (Andres et al. 1994, Running et al. 1995).

We initially classified African vegetation using the first three PCs of the $\overline{\text { NDVI }}$ into forest, savanna and grassland, and desert, using the K-means method (Spath 1980). We then computed the semivariance of the $\overline{\mathrm{NDVI}}$ in the N-S and E-W directions within types, focusing on the directional semivariance because of the strong anisotropy of vegetation density (at the continental scale) in Africa. Sills for the semivariance were typically higher E-W than N-S because of the influence of the strong N-S precipitation gradient in the Sahel. We set as a maximum threshold for grid cell radius the scale length (typically less than $200 \mathrm{~km}$ ) of the E-W semivariance, and we set as a minimum the grid cell size sufficient to obtain an adequate sampling of the BRF as determined by the number of unique geometrical configurations. Empirical evaluation of these joint constraints for this region resulted in grid cell sizes of $50 \mathrm{~km}$ radius. At this resolution, the spatial continuity is approximately isotropic, so the choice of circular grid cells is appropriate. Figure $5.5 \mathrm{~b}$ shows the transect of overlapping grid cells used in this analysis. We adopted the convention of numbering the cells from 1-15 starting at the northernmost cell. The discrete classification (for the entire continent) mentioned above 


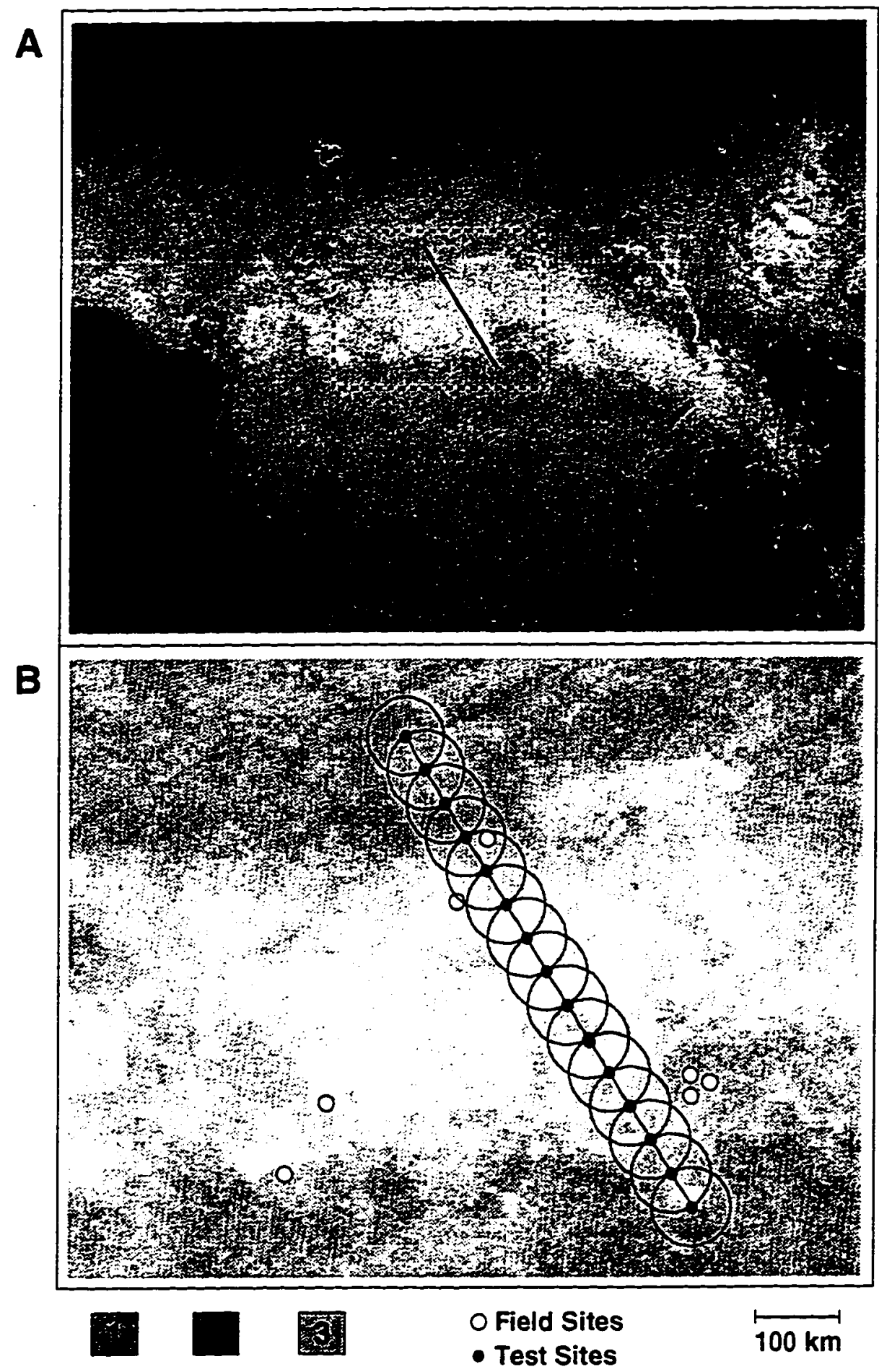

Figure 5.5 Phenological characterization of Africa. (a) Principal components 1,2 , and 3 as a mixture of red, green, and blue. Inset is $2 b$, showing the transect line. (b) The transect of 15 "cells" from grassland (blue pixels) to evergreen forest (orange pixels). Also shown are field sites where soil/litter reflectance was measured. 
resulted in this transect being broken into two classes, with the between-class line falling very close to cell 11 . We thus refer to cells $1-10$ as being a savanna/grassland mosaic, cells $12-15$ as being evergreen/deciduous forest, and cell 11 as "transitional".

\section{Leaf and Soil Oprical Measurements}

It is useful to obtain relationships between model parameters in order to reduce the number of free parameters in the inversion. This can be accomplished for a dependent variable by specifying a functional relationship between it and an independent variable, thus each time that variable is referenced during the optimization, it is assigned a value according to the prescribed function. We identified the leaf and soil/background optics variables as being most appropriate for data reduction because of the typically strong spectral autocorrelation in their reflectance/transmittance spectra.

An integrating sphere was used to measure the leaf optical properties of a set of savanna plants that were grown in a greenhouse. The leaves were of varying age and morphology. The measured reflectance and transmittance spectra were convolved with the AVHRR sensor bandwidths and the solar spectrum to produce visible (channel 1) and NIR (channel 2) values for reflectance and transmittance. Each point on Fig. 5.6 represents one of these reduced-spectral measurements. The existence of a large amount of correlation in these data led us to search for a way to represent the four leaf optical parameters with a single variable. Using the pairwise lines of best fit (solid lines in Fig. 5.6) is not a self-consistent approach. Principal components analysis yielded a 60 percent explanation of variance by the first PC. Thus, we used the first PC of these data to express visible leaf transmittance and NIR leaf reflectance and transmittance as a linear function of visible leaf reflectance. The resulting relationships are shown as dashed lines in Fig. 5.6 and represent the best way (in the least 
A

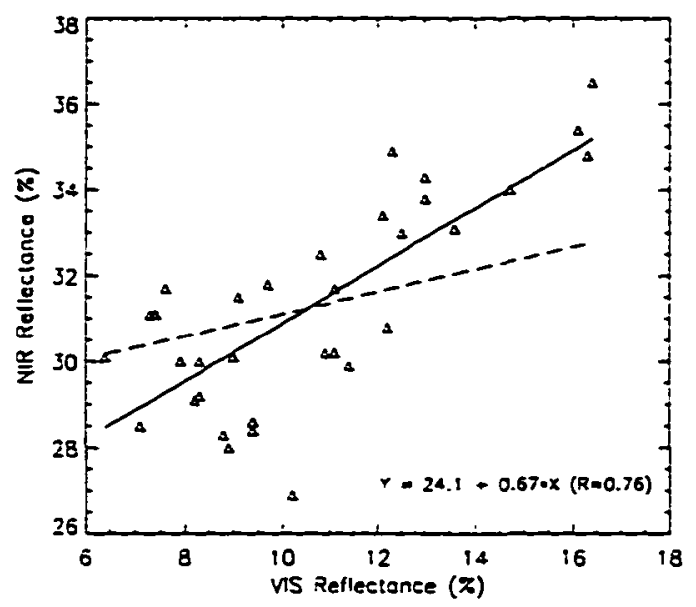

C

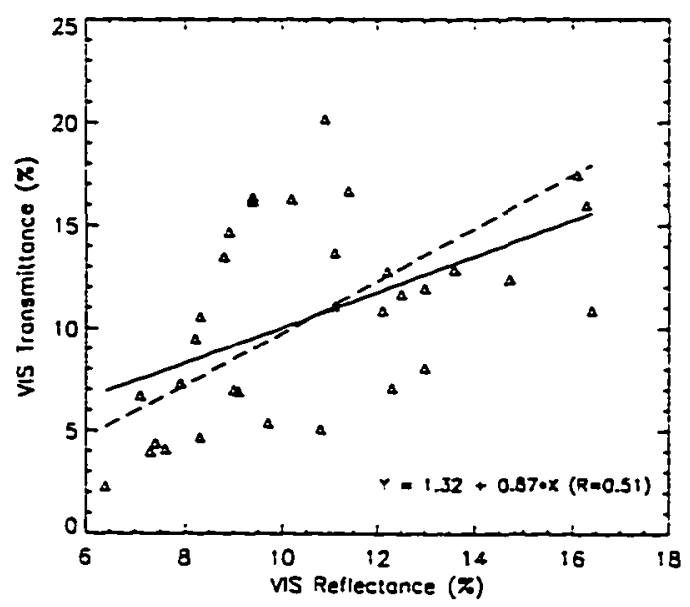

B

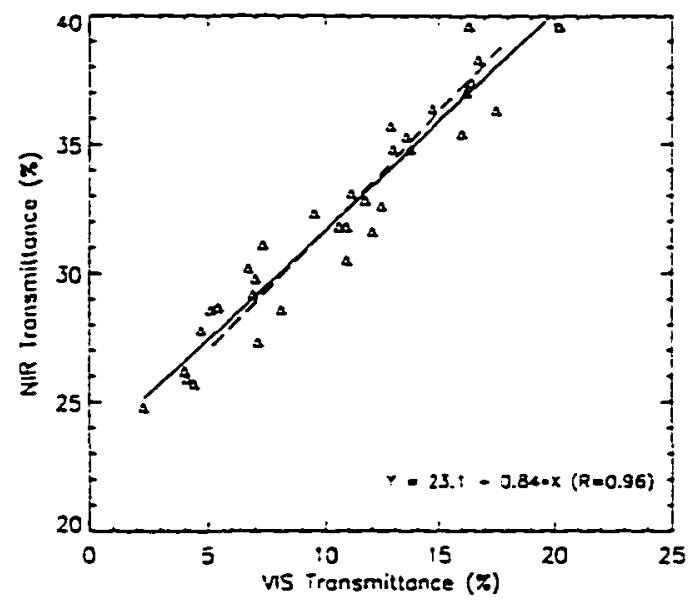

$D$

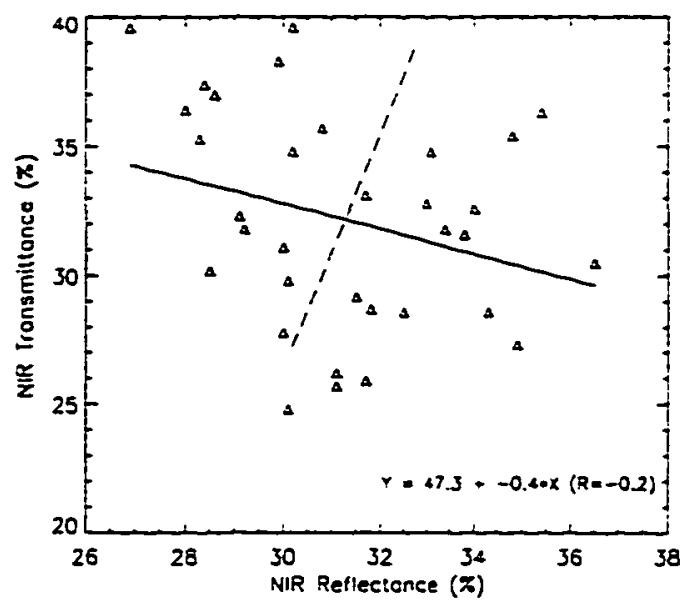

Figure 5.6 Leaf reflectance and transmittance data, convolved to the four leaf optical variables used in SAIL-2 and plotted against one another. The solid line is the line of best fit and the dashed line is the relationship obtained by writing all four variables in terms of the first PC of the leaf data (Appendix B). 


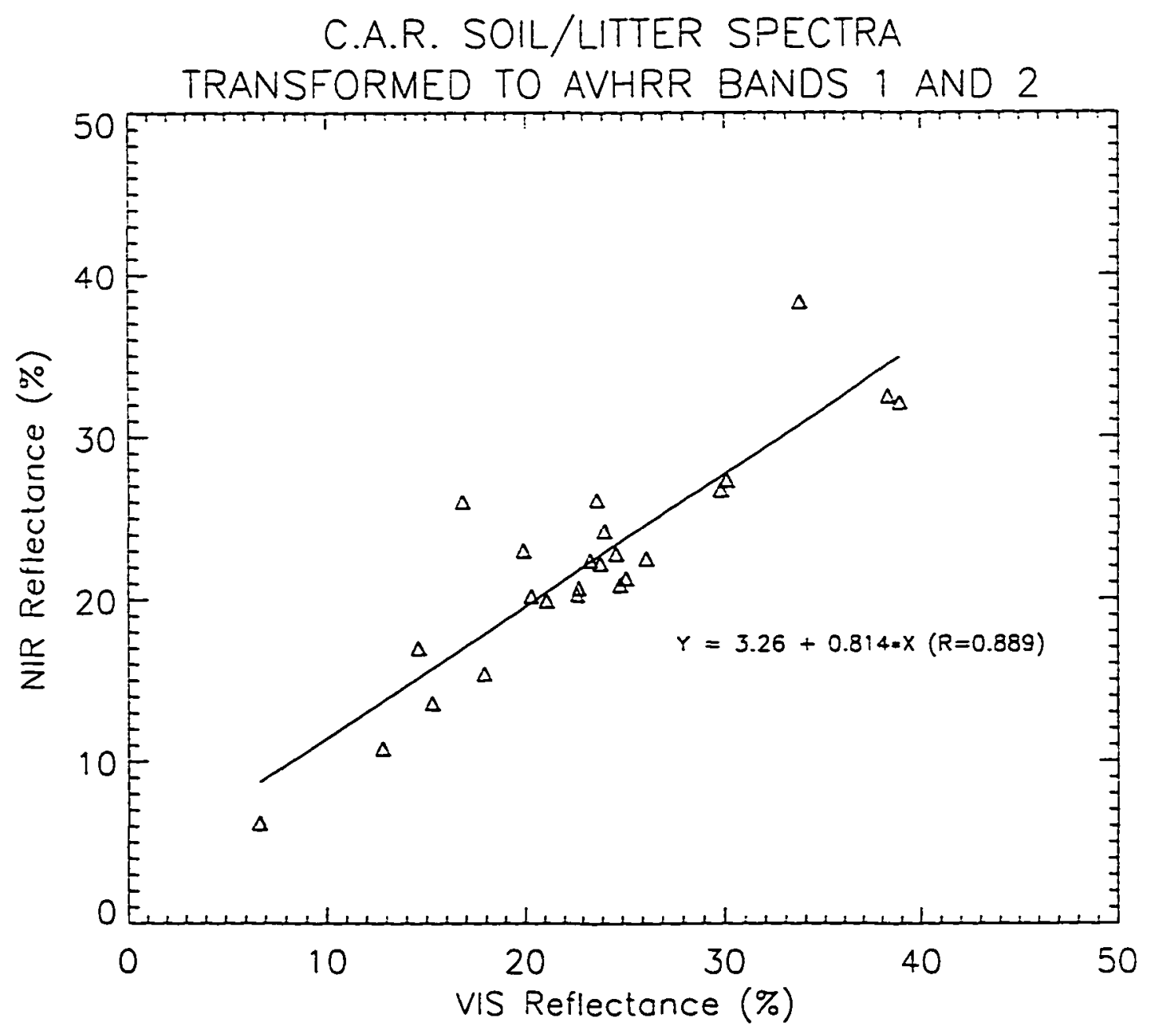

Figure 5.7 Similar to Fig. 5.6 except for field measured soil spectra. The line of best fit was used in the inversion. 
squares sense) to represent all the leaf optical parameters with one free variable.

The same technique was used for soil/litter reflectance. In January-February, 1995. we(co-authors EWS and ATH) visited a series of sites in the Central African Republic (C.A.R.) spanning the forest-savanna-grassland transition (Fig. 5.5b). LAI and soil/litter reflectance were measured at each site, including background spectra from areas that were recently burned. Wet-soil reflectance was also measured at each site. The combined soil and litter reflectance data were transformed into AVHRR-equivalent values using the same convolution method as for leaves. Using the transformed data, we developed a relationship between soil reflectance in the visible and NIR wavelength regions (Fig. 5.7). The soil line function, together with the leaf optics function, effectively reduce six of the model parameters (four for leaves, two for soil) down to two model parameters.

\section{Inversion Methodology}

We then extracted synthetic BRFs from a transect of grid cells in the C.A.R. (Fig. 5.5a.b) for all months of 1986 . The transect is $800 \mathrm{~km}$ long and consists of 15 cells; each cell is approximately $50 \mathrm{~km}$ in radius and consists of 121 Pathfinder $(8 \times 8 \mathrm{~km})$ pixels. This focused study region represents an ecological gradient from grassland in the north to evergreen tropical forest in the south, with mixtures of grassland, savanna, and woodland in between.

Multiple, geometrically unique observations of a target are required in order to use the bidirectional information to retrieve parameters, so this technique has operational value only if each cell contains pixels with significantly different sun-sensor geometry, and if the degree of sampling is consistent over time. This is true for the cells on our transect, as demonstrated in Fig. 5.8a for cell number 1. Sun-sensor phase angle $\alpha$ is a proxy for the full geometrical 
configuration ${ }^{*}$ (Fig. 5.8b shows the September geometries), so we have color-coded all points in Fig. 5.8a-d by the phase angle. Figure $5.8 \mathrm{c}$ is the realization of an actual BRF; pixels with similar geometry have similar reflectance values. In fact, this is a fairly typical profile, showing high backscatter reflectance, and lower reflectance in the forward scatter directions and at nadir.

Outlier pixels within grid cells were rejected on the basis of a similarity criterion, following the PC characterization of Africa. This effectively excluded lakes, villages, some river corridors, and vegetated areas that differ greatly from the dominant local structuralphenological type. The first three PCs of the $\overline{\text { NDVI }}$ data were used to apply a consistent requirement of similarity to each cell. Any pixel that differed from the modal $\mathrm{PC}_{i}$ value (for $i=1,2,3$ ) by more that 10 percent of the approximate total range of $\mathrm{PC}_{i}$ values for Africa was excluded. No more than half of the pixels were excluded from any given cell. On average, 70 percent of pixels were retained. Figure 5.9 shows the $\mathrm{PCl}-3$ values and the rejected pixels for a typical cell (number 1).

A number of free-parameter retrievals were performed on the transect of AVHRR data for 1986. We used three fixed-parameter sets, shown in Table 5.1 as G1, G2, and F. Parameters in each configuration could be free and bounded, fixed at different values in an ensemble, constant, or functionally-linked to another variable. The three parameter configurations in this experiment correspond to an idealized grassland in the dry season (G1; more dead grass than live grass), a grassland in the wet season (G2; mostly green grass), and an ideal forest (F). For the grassland sets, stems were assumed to be senescent leaves and thus the relationship between NPV optical variables is the same as for leaves. Also, having the same shape as green leaves, they are assigned the same leaf hot-spot parameter. The main difference

\footnotetext{
$\cos (\alpha)=\cos (\theta) \cos \left(\theta^{\prime}\right)+\sin (\theta) \sin \left(\theta^{\prime}\right) \cos (\psi)$
} 


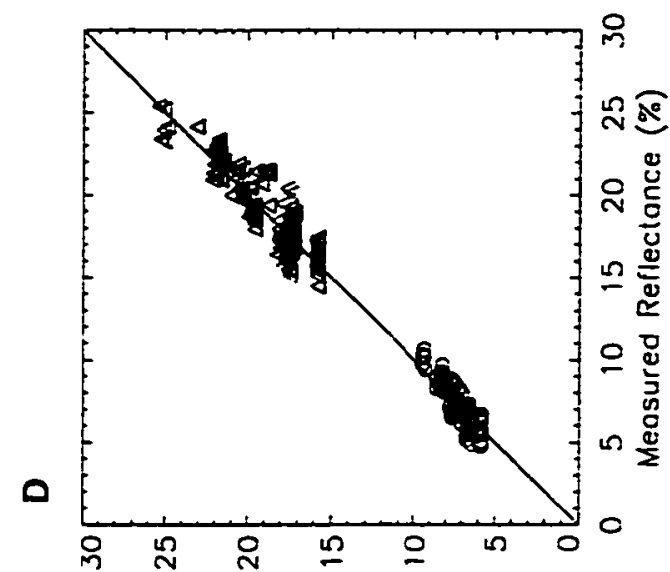

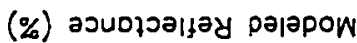

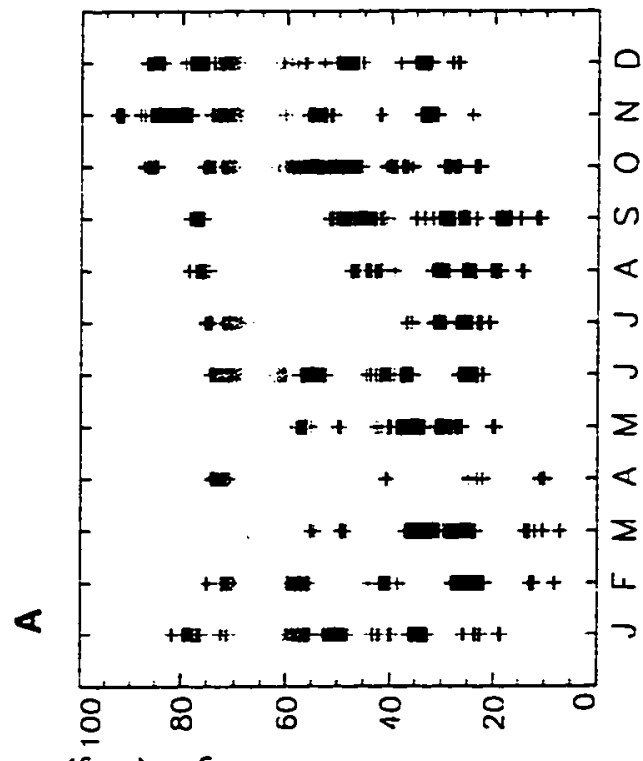

(6ap) a15ü asoud josuas-ung

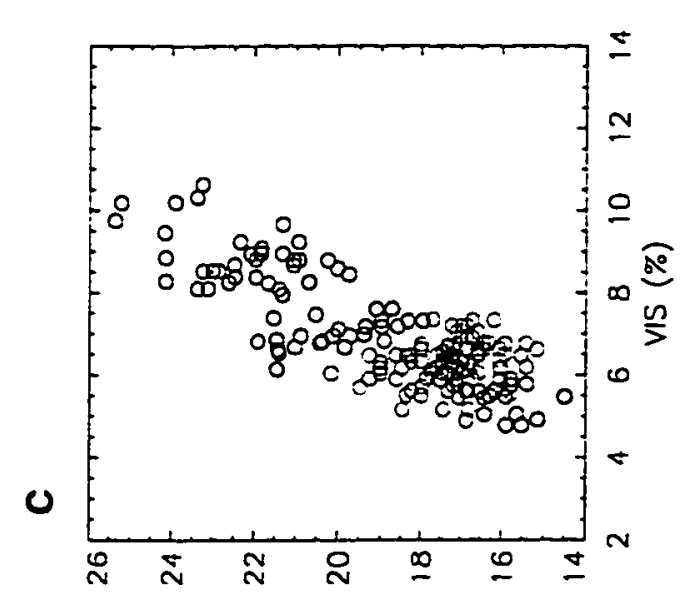

(\%) $\operatorname{diN}$

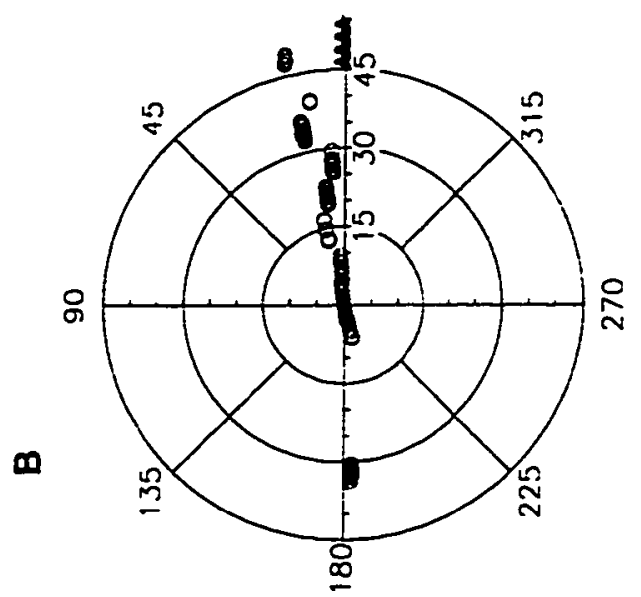

Figure 5.8 (a) Sun-sensor phase angle for a typical site as a function of time. (b) The full sunsensor geometry for September. (c) Measured visible and NIR reflectances for the same data as in (b). (d) Measured versus modeled reflectance after inversion on the data from (b) and (c). 


\section{Site 01, Spatial Analysis}

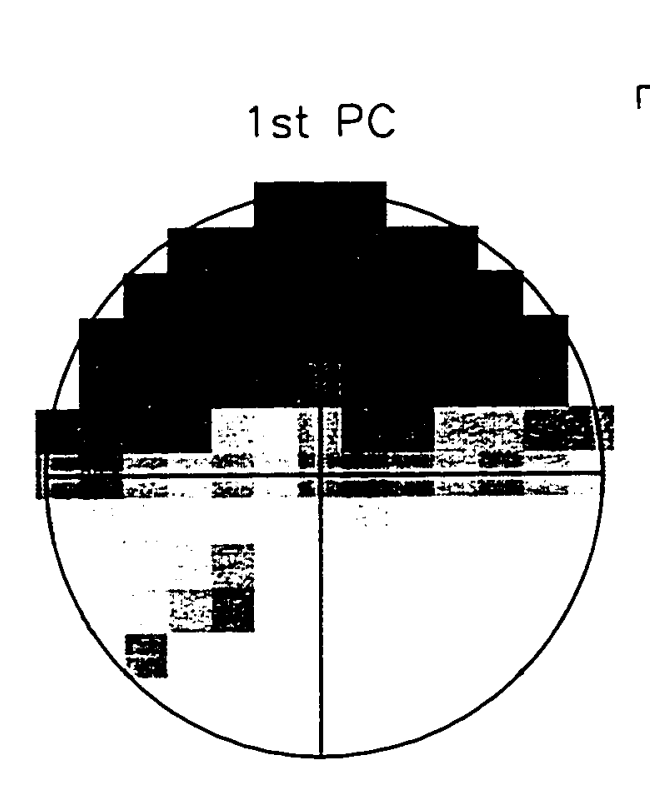

$50 \mathrm{~km}$
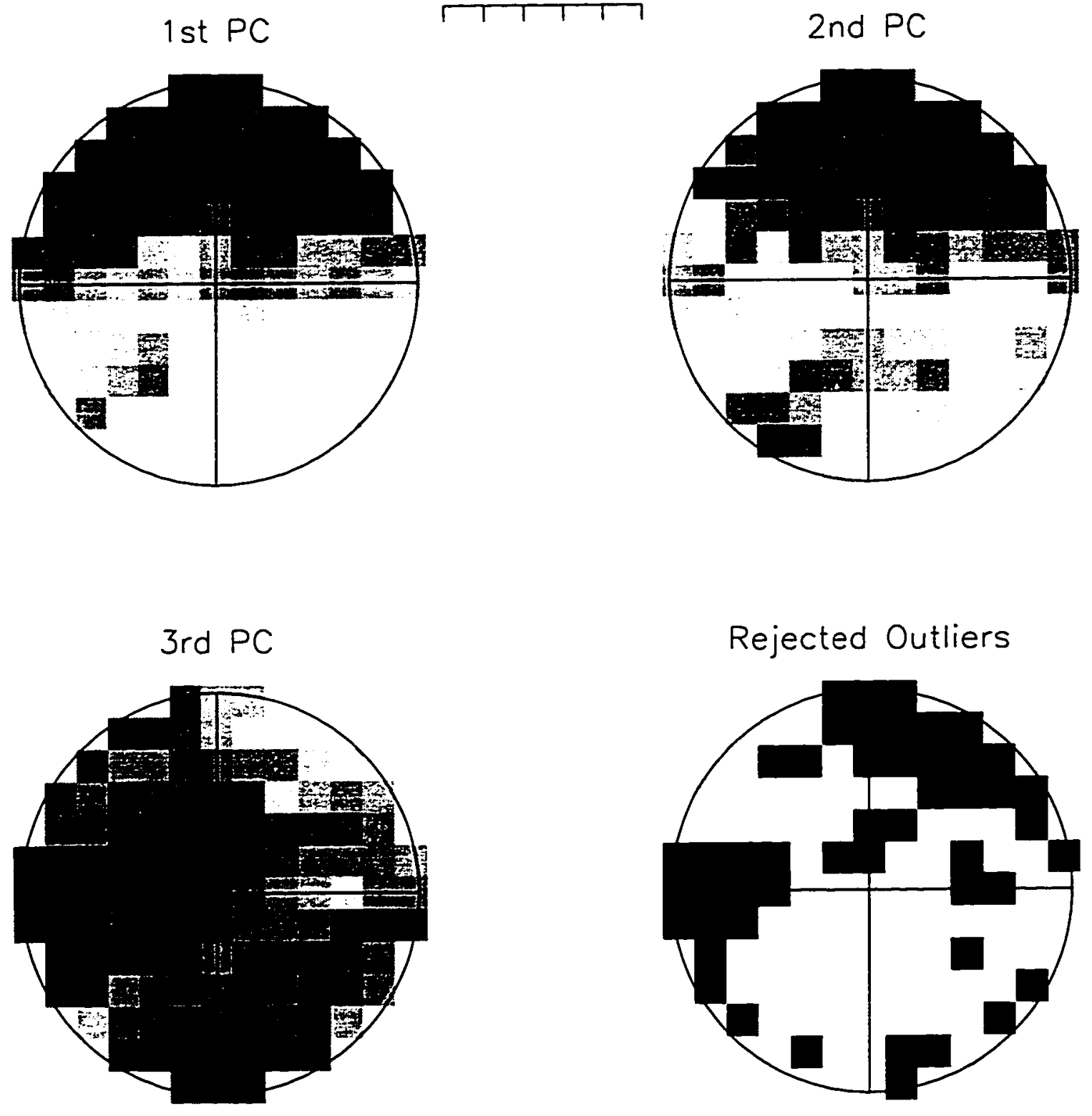

Figure 5.9 A typical cell characterized by a PC analysis of multi-temporal NDVI data. Individual pixels are rejected if they differ from the modal cell value for any PC by more than $10 \%$ of the continental range of values for that PC. 
between G1 and G2 is that the optical properties of NPV are free in the G1 inversion, and fixed in the G2 inversion (Table 5.1). This is because the relative abundance of NPV versus active leaves dictates, in part, the sensitivity of the optical parameters (Equation 5.15). In the "forest" set, stems are assumed to be tall (large hot-spot effect) and the green leaves small with respect to canopy height. Tree stems are also assumed to have zero transmittance.

For all the retrievals we inverted the SAIL-2 model on three free parameters: plant area index, stem fraction, and component (leaf or stem) reflectance in the visible. We used the optimization routine E04JAF (a quasi-Newton algorithm with simple bounds) from the Numerical Algorithm Group , to perform the inversions. Nearly all attempts at inverting with four free parameters failed, as did attempts where one of the three parameters above was fixed and any other parameter (e.g., soil reflectance) was free. By failure we mean that the optimization routine could not find a minimum of the merit function (Equation 5.14) given the parameter defaults and constraints. It is a convenient and important fact that when a parameter set is so unrealistic as not to allow a solution, the inversion returns with a null result. Our subsequent inference that the three "key" parameters above have the greatest BRF sensitivity (Equation 5.15) over the range of canopy conditions encountered in this study is consistent with the analysis of Privette et al. (1994).

A successful inversion, by definition, yields reflectances that agree as closely as possible, in the aggregate, with the measured AVHRR reflectance values. Figure $5.8 \mathrm{~d}$ shows a typical (described above) set of modeled vs. measured reflectances, corresponding to $R_{\mathrm{P}}$ and $R^{*}$ in Equation 5.14. The two clumps correspond to channel 1 (circles) and channel 2 (triangles). The 1:1 relationship in this figure is indicative of the success of the optimization, and the scatter reflects the magnitude of the merit function $E^{2}$ in Equation 5.14. This pattern indicates 
that the inversion has succeeded, and that the relationship between measured and modeled reflectances are well-behaved with respect to geometry.

\section{$\underline{5.3 .5 \text { Results }}$}

The SAIL-2 model was then inverted on the harvested AVHRR data to obtain $\mathbf{P}$ for each cell and for each month of 1986, using parameter configurations G1, G2, and F. Given the retrieved canopy structural and optical parameters, forward integrations (Equations 5.16 and 5.17) yielded daily total $F A P A R$ (Goward and Huemmrich 1992) and broad-band albedo. These secondary products are assumed to be more robust than the elements of $\mathbf{P}$ (e.g., LAI) because they integrate all of the model canopy properties and because they are radiative quantities. Our field measured LAI (sites shown in Fig. 5.5b) data are not directly comparable to the SAIL-2 retrievals of LAI $\left(P_{1} \cdot\left(1-P_{3}\right)\right)$, primarily because of the 9-year time difference, but they can provide a check on the results. In Jan. 1995, forest site LAIs were observed, ranging from $5.5 \pm 0.6$ in the evergreen tropical forests to $3.0 \pm 0.4$ in disturbed, upland sites. By comparison, retrieved forest LAI ranged from $4.6 \pm 2.0$ (cell 11) to $2.8 \pm 0.5$ (cell 14).

We merged the $f A P A R$ results for the three parameter configurations (G1, G2, and F) using the classification discussed above. The "grassland" parameterizations G1 and G2 were assumed to represent cells $1-10$, results for cells $12-15$ were taken from the "forest" $F$ inversions, and cell 11 was assigned the mean of the Gl/G2 and F results (Fig. 5.10a-b). Figures 5.10a and 5.10c also demonstrate the important success/failure phenomenon of the inversion. An unrealistic parameterization does not generally allow for a solution, so the "dry grass" G1 inversions fail during the peak growing season months, and the "green grass" G2 inversions are successful only during this period. The merged $f A P A R$ results for all sites for one year are shown in Fig. 5.11. The spatial-temporal patterns of daily total $f A P A R$ seen in Fig. 

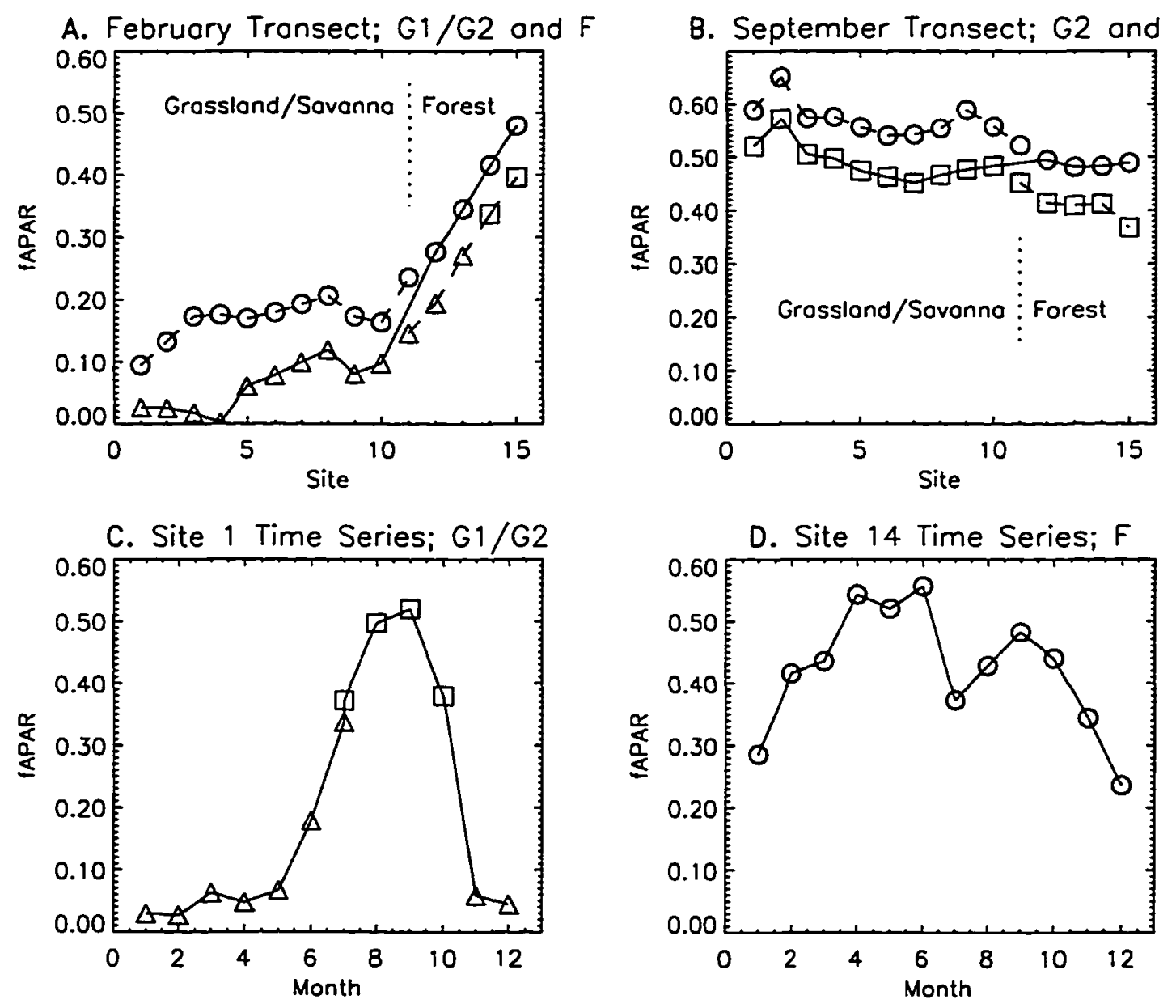

Figure 5.10 (a) Retrieved fAPAR along the transect in the dry season (February). The solid line connects the points with parameterizations that are appropriate for the vegetation type.

$\mathrm{Gl}=$ triangles, $\mathrm{G} 2$ = squares, $\mathrm{F}=$ circles (see text). (b) The same as (a) except for September.

(c) An fAPAR time series for the northernmost cell (1), using the G1 and G2 parameterizations.

(d) The same as (c) except for the southernmost cell. 


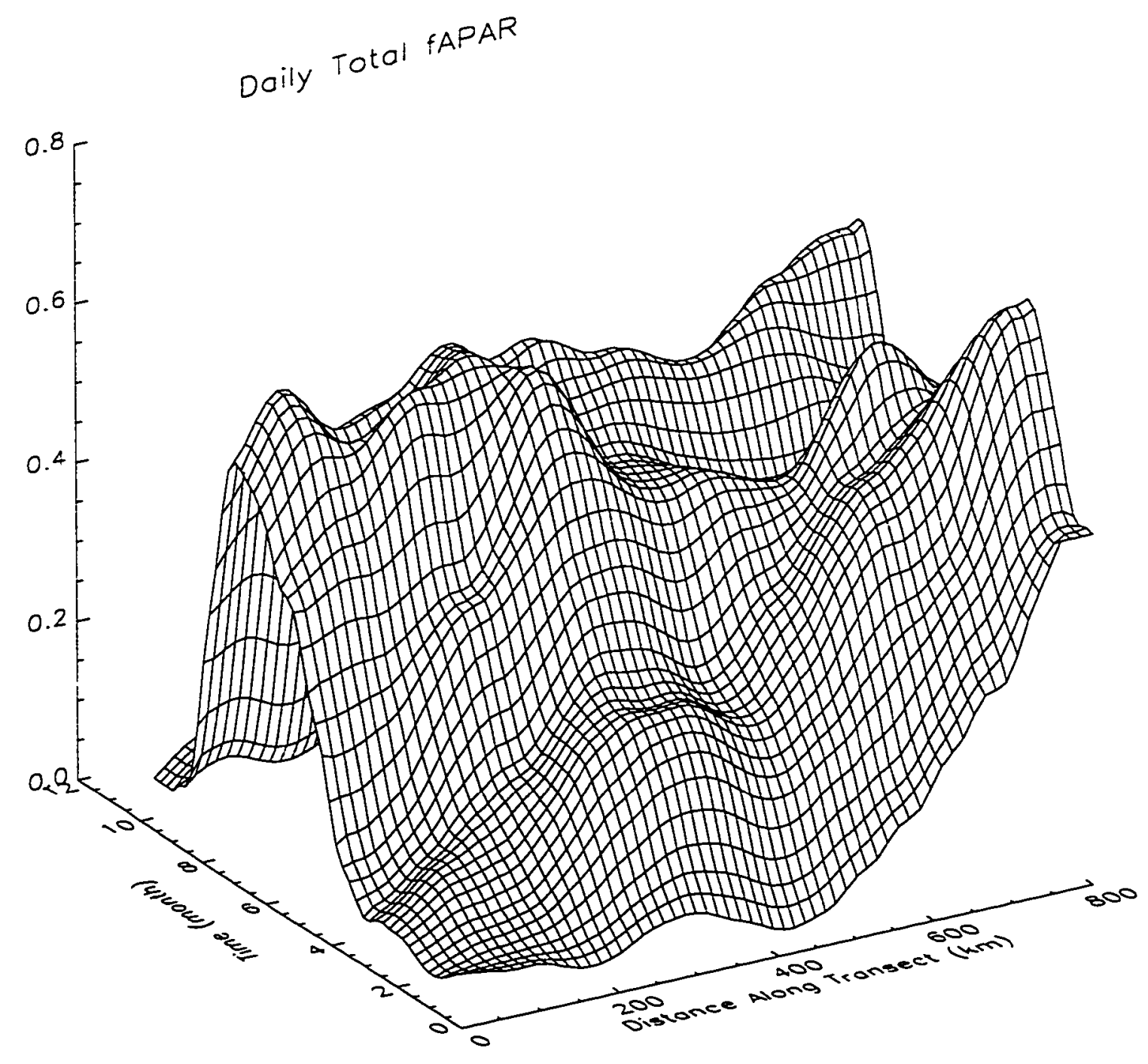

Figure 5.11 Retrieved $f A P A R$ values along the transect as a function of time. 
description discussed above). A slight bimodal seasonality can also be seen at the southern 5.10 and in Fig. 5.11 are a realistic depiction of the vegetation canopy dynamics in this region. The amplitude and timing of the seasonality decreases from north to south, producing a sharper $\mathrm{N}-\mathrm{S}$ gradient in the dry season than in the wet season (consistent with the geostatistical points, in agreement with analyses of Olsson and Eklund (1994).

We compared our $f A P A R$ results to those calculated using the VI-based method of Sellers et al. (1994) for the Simple Biosphere model (SiB). There is roughly a 1:1 agreement (Fig. 5.12a-b) between the two approaches, but with a moderate amount of scatter $\left(R^{2} \approx 0.88\right)$. Error bars for the Sellers et al. $f A P A R$ result from within-cell variation of NDVI while error bars on our estimates reflect the uncertainty propagated from the parameter ranges that were applied as part of an ensemble (Table 5.1). The forest NDVI was transformed into fAPAR using the equations for the SiB tropical evergreen class, and for the remaining cells, we used the SiB savanna equations (Fig. 5.12a) and the SiB grassland equations (Fig. 5.12b). A clear offset can be seen between the SAIL-2 and the Sellers et al. (1994) determination of $f A P A R$, as well as a strong dependence on which VI transformation was used to get the savanna/grassland points. Also, the difference becomes amplified at high $f A P A R$ values. The existence of a good relationship is possibly due to the fact that averaging VI values over a $50 \mathrm{~km}$ radius cell reduces the effects of variable sun-sensor geometry and background reflectance. Less correspondence would likely be observed if data from a multi-angle like MISR (Diner et al. 1989) was used, because less spatial averaging would be required in the inversion.

Finally, we evaluated our estimated land surface albedo for the transect against the SiB model-derived estimates of Dorman and Sellers (1989) and an approximation using METEOSAT (a narrow-band visible weather monitoring instrument) data (Becker et al. 1988) along a transect at $20^{\circ} \mathrm{E}$. Table 5.2 shows the albedo comparison for both ends of the transect 
A.
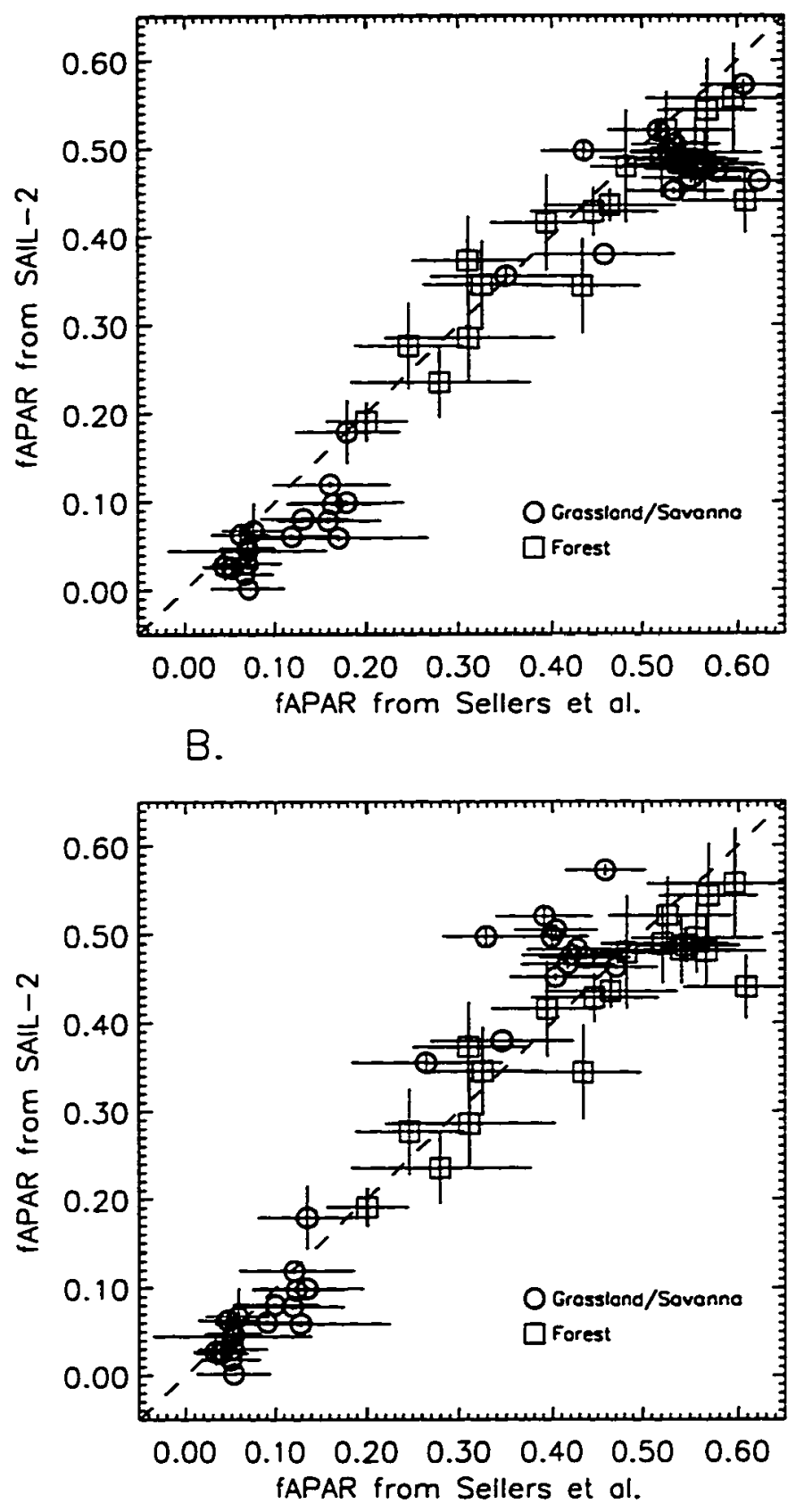

Figure 5.12 (a) A comparison of $f A P A R$ derived from the SAIL-2 inversion and $f A P A R$ using the vegetation index-based method of Sellers et al. (1994) for SiB forest (circles) and savanna (squares) types. (b) The same as (a) except using SiB forest (circles) and grassland (squares) types. 
Table 5.2 Broad-band albedo estimates from the SAIL-2 inversions compared to the SiB model (Dorman and Sellers 1989), and METEOSAT (Becker et al. 1988). Cell 1 is centered at $8^{\circ} \mathrm{N}$, and cell 15 is centered at $4^{\circ} \mathrm{N}$.

\begin{tabular}{lccc}
\hline Location/Time & SAIL-2 & SiB & METEOSAT \\
\hline Cell 1, January (dry) & 0.10 & $0.15-0.20$ & 0.09 \\
Cell 1, September (wet) & 0.15 & $0.10-0.15$ & $0.16-0.18$ \\
Cell 15, January & 0.11 & $0.15-0.20$ & 0.10 \\
Cell 15, September & 0.12 & $0.10-0.15$ & $0.16-0.17$ \\
\hline
\end{tabular}

at two times of the year. Compared to $\mathrm{SiB}$, our values are of comparable magnitude but they do not co-vary with respect to vegetation type and seasonality. However, the METEOSAT-derived albedo appears to show good agreement in magnitude and seasonality at both latitudes. Future efforts to compare our results to Earth Radiation Budget Experiment data will provide more insight into the accuracy of these estimates.

The two comparisons with $\mathrm{SiB}$ formulations do not constitute validation of our inverse modeling results. At this time, VI-based algorithms are the only other way to estimate biophysical parameters at regional-to-global scales. The comparison demonstrates that our results (using unconventional methods) are comparable to other estimates, but it reveals some intriguing differences. Further, though it is likely that the $f A P A R$ values derived from inverse modeling are more accurate than those using VI's because of their greater information content, the two techniques are suited for somewhat different applications. A VI-based approach is computationally fast, and more applicable for coarse resolution (e.g., GCM-scale) analyses. Inverse modeling applications are more computationally intensive, but are based on physical and ecological theory, and thus are more appropriate for finer-scale studies. Moreover, inverse modeling may feed back into the development of better VI algorithms, as is currently planned for the NASA-EOS MODIS instrument (S. Running, personal communication). 


\section{$\underline{5.3 .6 \text { Discussion }}$}

Satellite remote sensing allows for direct observation of the status of global vegetation at regular spatial and temporal intervals. This density of observation is important for monitoring terrestrial ecosystems because of the high spatial and temporal variability in climate forcing and because of the nonlinear response of terrestrial biogeochemistry - particularly factors relating to water and nutrient status. We have presented an algorithm for extraction of land surface biophysical information (LAI, $f A P A R$, and albedo) that is relevant for biogeochemical, ecological, and biophysical modeling, and that has the following unique characteristics:

1. It uses real satellite data and a physically-based model to retrieve parameters over large spatial areas. The SAIL-2 parameter set consists of physically meaningful quantities (Table 5.1) and leads to estimates of $f A P A R(\mathbf{x}, t)$ and albedo $(\mathbf{x}, t)$.

2. It simulates a multi-directional sampling of the BRF by gathering a spatial-temporal neighborhood of pixels with an assumed spatial continuity. This assumption is enforced by a principal components (or Fourier) decomposition analysis of a vegetation index climatology.

3. It allows for the incorporation of a priori ecological knowledge in the choice of parameter constraints and of the inversion mode (i.e., which parameters are held fixed, and which are free). This method highlights the importance of data describing soil and leaf optical properties keyed to global soils and vegetation databases. These data are readily but rarely measured.

4. It allows for direct incorporation of field measurements of plant canopy and surface soil/litter (background) characteristics, which typically reduce the number of free parameters in the inversion and may be applied over large areas, as in this study. 
There are major differences between our approach and VI-based, empirical approaches (Potter et al. 1993, Sellers et al. 1994, Ruimy et al. 1994). First, we use both optical channels simultaneously instead of combining them into one index. Second, we retrieve parameters for a cluster of pixels (one cell) instead of for each pixel, effectively trading lower resolution for increased accuracy. Third, we explicitly account for the two factors that are most responsible for the distortion of the vegetation signal: background spectral variation and bidirectional effects. Fourth, we account for PAR interception by non-photosynthetic vegetation which allows estimation of $f A P A R$ for leaves. Finally, with inverse modeling, the uncertainty associated with lack of knowledge of some surface characteristics (parameters) and the validity of ecologically-based assumptions may be quantified using fixed-parameter ensembles.

Our conclusion, based on these preliminary results, is that it is possible to retrieve land surface parameters from remotely-sensed data using RT model inversions. Although we used a relatively simple RT model (SAIL), this method could easily be adapted to more sophisticated ones (Privette et al. In press). However, more complex models generally have more parameters; thus they may not be advantageous unless (1) the remote sensing data contains more BRF information per unit area and less atmospheric noise (e.g., MISR), or (2) more ecological constraints are available. Because our method is based on radiative transfer theory, it is probably more accurate than those achieved using VI-based algorithms (except those that are highly tuned), but this has yet to be established experimentally. Our method requires no empirical calibration between reflectance (or a VI) and any of the estimated variables: fAPAR, albedo, or LAI. Field validation is essential, but difficult, because of scaling issues associated with the estimation of LAI, albedo, or $f A P A R$ over an areas as large as $2500 \mathrm{~km}^{2}$. One way to attack the problem of verification is by using spectral unmixing techniques and data from other. higher resolution sensors to bridge the gap between field measurements and continental scale 
(e.g., AVHRR) data. These methods could also feed back to the large scale parameter estimation by providing better quantification of canopy component properties. Indeed, the combination of inversion and unmixing techniques has been proposed as a "paradigm shift" in the remote sensing of vegetation biophysics (Hall et al. 1995).

Although the AVHRR Pathfinder data set is carefully processed, there are two "minor" problems with the first release of the data (Prince and Goward 1996): (1) the incident solar radiation was not adjusted for solar zenith angle; and (2) there was an error in the correction for atmospheric Rayleigh and ozone scattering. These translate into RMS reflectance errors for a typical composite of as much as $1-3 \%$ (M. James, official communication). Moreover, the data have not been corrected for the effects of scattering by atmospheric aerosols, which can be large. Further processing of Pathfinder data is currently being planned to correct these problems, which could lead to improved accuracy of our analysis. We feel that the successful inversions presented in this study, using imperfect data, represent an extreme test of our algorithm.

This inverse modeling method can easily be used in the EOS framework, particularly with the MODIS and MISR sensors. Data from both sensors will be atmospherically corrected and calibrated with state-of-the-art techniques, and sampling rates of MODIS are comparable to those of AVHRR (Sellers and Schimel 1993). While MISR repeat samples are up to nine days apart, that sensor's ability to measure a target at nine angles per satellite pass will likely result in smaller (and less heterogeneous) aggregation cells. Both sensors have spatial resolutions of less than one kilometer at nadir over several visible and NIR bands. The higher resolution sampling of solar and PAR spectral wavelengths by MODIS should allow for better estimation of land surface properties such as $f A P A R$ and albedo. 
With algorithms such as the one presented in this section, inversion methods could soon be mature enough for large-scale applications. Automation of some decisions that are based on geography, climate, or time of year. will be possible in conjunction with consideration of ecological factors. Because they are easily incorporated into inversion methods, advances in the knowledge of ecosystems, radiative transfer, or numerical methods as well as refinement of global ecological and soils databases, will translate directly into a more accurate retrieval of biophysical parameters and may lead to a better understanding of the role of the terrestrial biosphere in the Earth system.

\subsection{Toward Remote Sensing Data Assimilation for Terrestrial Modeling}

Optical remote sensing data are beginning to be used in concert with models of land surface biophysics and biogeochemistry. Because of the observation that NDVI and SR (Equations 5.6 and 5.9) are correlated with $F A P A R$, this is the primary relationship that is exploited. Vegetation indices, however, may be more powerful as a validation tool than as a predictor because uncertainties in the data, uncertainties in the transformation to $f A P A R$, and circularity inherent in the logic of the relationship between fAPAR and net canopy photosynthesis (see Section 4.2 and Field 1991). Further, inverse radiative transfer modeling provides a framework for estimating parameters in a way that is compatible with terrestrial process model parameters, and one that does not override the model's representation of reality. We will briefly review in this section two examples of using AVHRR-VI data to drive terrestrial models, and one example of an inverse technique (using field BRF data) that suggests the possible role of remote sensing data assimilation in terrestrial modeling.

Potter et al. (1993) developed a model of terrestrial cycling of carbon and nitrogen among vegetation and soil components. The model is similar in structure to the Century model 
(Parton et al. 1987), and in fact the soil organic matter transformation rates are based on Century. The model simulates net primary productivity NPP in the following way:

$$
\mathrm{NPP}=\varepsilon \cdot A P A R,
$$

where $\varepsilon$ is a total conversion efficiency that incorporates biochemical rates of light utilization, and stress factors (water, nutrients, and temperature), and APAR is the total PAR absorbed which is equal to $E_{s} \cdot f A P A R$ (see Equation 5.7). The fractional PAR absorbed is based on work by Sellers et al. (1992), and is given by

$$
f A P A R=\min \left(\frac{S R}{S R_{\max }-S R_{\min }}-\frac{S R_{\min }}{S R_{\max }-S R_{\min }}, 0.95\right)
$$

where the lower bound for the simple ratio $S R_{\min }$ is value at which it is assumed no PAR is absorbed and is based on the observation of unvegetated land surfaces. Conversely, $S R_{\max }$ is ecosystem type dependent and reflects the value at which all incident PAR is absorbed. Equation 5.20 represents the link to remote sensing for a model that is otherwise based on ecosystem carbon and nitrogen dynamics.

In a similar effort, Ruimy et al. (1994) developed a model that was more oriented toward flux calculations (i.e., stocks of carbon were not emphasized). Their study explored the sensitivity of net ecosystem exchange to various representations for the relationship between NDVI and $f A P A R$, as well as values for the conversion efficiency $\varepsilon$. Their best estimate of global integral NPP ( $\left.60 \mathrm{Pg} \mathrm{C} \mathrm{yr}^{-1}\right)$ agrees closely with other values from the literature, but the mean values per biome do not correspond as well with other published estimates. Ruimy et al. 
(1994) argue that their estimate is more accurate that those that do not use NDVI to predict NPP, but there is considerable variability in their results associated with different assumptions about $\varepsilon$ and the NDVI-fAPAR relationship. In a subsequent study (Maisongrande et al. 1995) using the same model, interannual variability in NPP (and NEP) was inferred from a multi-year AVHRR-NDVI data set. The authors claim that effects of climate cycles (e.g., El NinoSouthern Oscillation) can be seen in the resulting flux estimates. If this is true, then it represents a powerful application of remote sensing data (but see Chapter 4).

Using remote sensing derived estimates of $f A P A R$ to drive ecosystem models may not be the most effective way to incorporate such data into process studies. This is primarily because there is well-developed theory linking climate and biome-based potential NPP to derive the dynamics of whole ecosystem carbon fluxes. Also, observations of bulk canopy radiative properties reflect both actual and potential photosynthesis because both LAI and leaf optics contribute to the remotely sensed signal. Leaf area index is related to potential photosynthesis, but stresses during the growing season can result in lower LAI. Leaf optical properties (governed in part by chlorophyll content) also change in response to stresses that would be “double-counted" upon application of Equation 5.19.

Schimel et al. (In press b) discussed issues related to validating spatial patterns of model prediction using remote sensing. They examined model predictions by the Vegetation/Ecosystem Modeling and Analysis Project of net primary productivity. One goal was to check the realism of the spatial variability of the model estimates using long-term monthly mean NDVI ( $\overline{\mathrm{NDVI}}$; Equation 5.18) for each $0.5^{\circ} \times 0.5^{\circ}$ grid cell, under the assumption that NPP and NDVI are related by Equations 5.19 and 5.20. They found that within biomes, NPP and $\overline{\text { NDVI }}$ are uncorrelated. This comparison was made using the mean deviates of both quantities (with each grid cell value corrected by the mean of values from all the grid 
cells in an ecosystem type). Correlations with the original, uncorrected data were relatively high $\left(R^{2}=0.6-0.7\right)$. Thus the models appeared to be representing across-biome patterns of NPP, but no conclusion could be made about within-biome variability.

In the numerical weather prediction (NWP) community, there is an established paradigm for using a priori knowledge of processes (embodied by a model) to interpret data. This is accomplished by a set of techniques called data assimilation. Data assimilation may also be thought of as a formal methodology for constraining a model simulation to be as consistent as possible with observations. The basis for the constraint is the calculation by the model of the observed parameters at the same location and time. Though there are various techniques differing in mathematical detail, typically the time-evolution of state variables is specified by the model equations, and model parameter values are adjusted iteratively until satisfactory degree of agreement is achieved. This process is conceptually equivalent to the methods used in inverse radiative transfer modeling (Section 5.3).

Bouman (1992) has used field-measured optical reflectance and microwave backscatter of an agricultural canopy in a quasi-assimilative mode to calculate crop growth and yield. Physical models of radiative transfer (for optical and microwave signals) were linked to a crop growth model via canopy structural variables (e.g., LAI). Appropriate meteorological data were prescribed. An optimization procedure was used to find the parameter set that yielded the lowest absolute error in reflectance/backscatter averaged over the growing season. They found that using either the microwave or optical data resulted in more accurate predictions of crop yield, and using both types of data together significantly further improved the estimates.

Considering the success of Bouman (1992) and the arguments above, we propose that coupling a radiative transfer model like SAIL-2 to a biophysical or biogeochemical model could enable assimilation of optical data (e.g., from AVHRR) for calculation of landscape $\mathrm{CO}_{2}$ 
fluxes. It is likely there is not enough information in the satellite data to completely determine the patterns of terrestrial NPP (e.g., Ruimy et al. 1994), especially because of uncertainty in the application of rules like Equations 5.19 and 5.20, and because of the complex interactions between vegetation, soils, and climate. Many of these effects are not manifest in canopy architecture or optics in an immediate or straightforward way (Chapter 4).

\subsection{Summary}

Key Earth system variables can be obtained from satellite data by integrating appropriate processing, interpretation. and analysis. In order to interpret the wealth of information about the terrestrial biosphere contained in satellite data, there are three major considerations, all of which can require considerable effort. First, a model is needed (either empirically or physically based) to relate the measurements made at the satellite to biophysical or ecological quantities at the land surface. Second, factors that are unrelated to the land surface, which contaminate the signal, must be accounted for. Third, though it is not widely regarded in the literature, it is likely that a priori knowledge of land surface characteristics

(e.g., terrain, species composition, canopy architecture, degree of heterogeneity. phenology, soil type) is necessary for accurate retrieval of the desired parameters.

Vegetation indices can be used with any data that contain multi-spectral information, and geometrical dependence can be exploited with any data that contain significant angular variation. AVHRR data is applicable to both categories. The difference between these two variables is that the spectral sampling is a predetermined property of the instrument, while instruments with systematic geometrical sampling are only proposed, e.g., MISR (Diner et al. 1989). Thus, in principle, one could envision the use of geometrical and spectral indices. Analogously, inverse modeling simply requires multiple measurements that contain variability 
in any independent variable in concert with a model that can produce reflectance estimates as a function of those variables. In the primary work of this chapter (Section 5.3), we have demonstrated a method for using AVHRR optical reflectance as a function of both independent variables to estimate ecosystem biophysical properties.

One of the important uses for satellite-estimated land surface parameters is to assist process modeling of terrestrial biogeochemistry (i.e., prediction of large-scale fluxes of carbon, nutrients, and water). An established technique is to assume that the optical measurements, by way of a VI or inversion-derived $f A P A R$ can be used to fix the value of potential photosynthesis in such calculations. However, models are already conceptually equivalent to schemes for integrating and interpolating ecological and meteorological data. Thus the framework for biogeochemical modeling is adaptable to including remote sensing data in a more complementary way. For reasons discussed above, we propose that remote sensing "data assimilation" techniques (which require consistency between model and data) be used for assessment of intra- and interannual carbon (and other trace gas) fluxes between the terrestrial biosphere and the atmosphere. 


\section{CHAPTER 6}

\section{CONCLUSIONS}

\subsection{Review of chapter contributions}

In this dissertation, we presented original work from ongoing collaborative and interdisciplinary research. We discussed methods for studying the nature of global terrestrial biogeochemical dynamics as perturbed by human activity, focusing on annual-to-centennial time scales. Our primary methods involved using global models and observations. Spatial resolution was also an important factor in this work; we considered global scale analyses that were both spatially explicit (e.g., Chapters 3 and 4), and averaged over space (e.g., Chapter 2). The finest spatial resolution considered in this work was in Chapter 5, where extracted information from $8 \times 8$ kilometer grid cells (which were re-aggregated to approximately 0.5 degree resolution). In the course of this work, a number of conclusions relating to the terrestrial biosphere and global change were put forth. We briefly review these below.

1. Enhanced terrestrial uptake over the last two hundred years can be inferred from aggregated models of the global carbon cycle. This sink is significant (perhaps as large or larger than the oceanic sink) and can be explained, in part, by direct effects of increasing concentrations of atmospheric $\mathrm{CO}_{2}$. Further, timescales for the persistence of anthropogenic $\mathrm{CO}_{2}$ in the atmosphere are very sensitive to the activity of the terrestrial biosphere, including the nature and controls over mechanisms which comprise the so-called "missing sink."

2. Terrestrial fertilization by anthropogenic nitrogen deposition is (and has been) likely a significant component of the global carbon budget of "excess" $\mathrm{CO}_{2}$, and has important effects 
on the coupling between global carbon and nitrogen cycles. Uncertainties are associated principally with the spatial patterns of deposition and details of terrestrial biogeochemical response to exposure to chronic $\mathrm{N}$ and other pollutants. The persistence of nitrogen fertilization (like direct $\mathrm{CO}_{2}$ fertilization) is constrained by negative feedbacks involving pollutant exposure and "N-saturation."

3. The response of net terrestrial carbon exchange to climate variability (and likely other perturbations) occurs over multiple years and is governed by internal biogeochemical regulation, which depends on the prior state of the ecosystem and the characteristic turnover times of soil and vegetation components. Because terrestrial ecosystems are heterogeneous at all spatial scales, climate- $\mathrm{CO}_{2}$ relationships are the result of the superposition of local responses to transient climate anomalies. In addition, these responses are highly affected by land-use because of its impact on ecosystem biogeochemistry and the distribution of vegetation types with specific resource use efficiencies.

4. It is possible to retrieve land-surface biophysical parameters over large regions from satellite data using radiative transfer models in concert with site data and geographically referenced vegetation and soils databases. This method accounts for factors (i.e., bidirectional and background effects) which confound more simplistic analyses of reflectance data, and it provides a framework for incorporating extensive and readily available a priori ecological knowledge. The algorithm we present has the potential to provide more accurate constraints on terrestrial process models which produce estimates of the spatial and temporal patterns of net carbon exchange.

\subsection{Future work}

The studies presented in the previous chapters generally raised more questions than they answered. Thus, they all represent continuing projects that, though presently separate, 
have been converging, and will likely continue to converge in many respects. Below we outline some prospects for future work that are based on the continuation of the developments discussed in this dissertation.

Aggregated global carbon cycle models (Chapter 2) continue to be useful despite their simplicity, as demonstrated in the most recent IPCC (1995) report. We plan to bring the Moore and Braswell (1994) model up to date by including temperature-dependent component turnover times, by allowing for a sink mechanism that is not controlled directly by atmospheric $\mathrm{CO}_{2}$ concentrations, and by re-investigating the ${ }^{13} \mathrm{C}$ and ${ }^{14} \mathrm{C}$ constraints on the partitioning of excess carbon amongst reservoirs. Further, we plan to make the model more readily available as an educational tool; it will be used in an upcoming IGBP/GAIM workshop to be held in Mombasa. Kenya in 1997.

The global nitrogen cycle is gaining wide recognition for its importance in both global biogeochemistry and climate change. The work in Chapter 3 represents some of the initial results (first and second publications) of a collaborative project with E.A. Holland, A.R. Townsend, and other investigators. The third phase of the project involves producing a gridded data set $\left(0.5 \times 0.5\right.$ degree resolution) of wet and dry $\mathrm{NO}_{\mathrm{y}}$ and $\mathrm{NH}_{\mathrm{x}}$ deposition for the continental US. This data set will be used for validation of the modeled deposition fields and as input for another modeling study. We plan to use the Century model to validate (and likely modify) the simplistic parameterizations in NDEP.

Many questions were raised in Chapter 3 about the nature of the interannual response of terrestrial ecosystems (and carbon exchange) to climate perturbations. While the observational data qualitatively supported the modeling analyses, we necessarily stopped short of presenting a unified theory. We plan to take a closer look at the spatial and temporal structure of the data, expanding our analysis to the less precisely known precipitation record. 
This work will benefit from continued collaboration with E. Linder (University of New Hampshire). Further, we plan to perform model-based analyses (using Century) that are more directly compatible with the data. This will involve a global climate perturbation study (we have studied site responses only up to this point) and the consideration of implications for plant canopy conversion efficiencies (for comparison to satellite optical measurements).

We have argued that land-surface information retrieved using radiative transfer model inversion are potentially more accurate because of the way they use spectral and bidirectional information, and because they facilitate use of generalizable knowledge of optical and structural properties of ecosystems. This assertion has not been formally tested. We are proposing to perform an end-to-end validation of the algorithm presented in Chapter 5 by linking field data to coincident bidirectional observations made by an airborne spectroradiometer. This work will be done in collaboration with G. Asner (University of Colorado). D. Schimel, and B. Moore. Further, we will explore in detail the prospects of using MODIS, and especially MISR data in the inversion algorithm. 


\section{APPENDIX A}

\section{THE SAIL MODEL EQUATIONS}

The SAIL model formulation treats plant canopy components as a "turbid medium", i.e., an idealized distribution of scattering elements. As such, it has a rather distinct lineage in the theory of radiative transfer. We will outline the progression of the theory (essentially a series of small modifications) leading to the SAIL version implemented by Braswell et al. (1996; Section 5.3). We will use the notation of Verhoef (1984) as much as possible throughout. The fundamental notions underlying the SAIL model can be traced back to work by Schuster (1905), who described the spatial dependence (in one dimension) of radiant energy in a "foggy" medium as being composed of two diffuse fluxes of light moving in opposite directions. He based the description on the scattering and absorption of light by an infinitesimal layer $d x$ of the medium (in this case, a stellar atmosphere), thus the forward $E_{\text {. and backward }}$ $E_{+}$fluxes are given by:

$$
\begin{aligned}
& \frac{d E_{-}}{d x}=\kappa J-\kappa E_{-}+\frac{1}{2} \nu E_{+}-\frac{1}{2} \nu E_{-} \\
& \frac{d E_{+}}{d x}=-\left(\kappa J-\kappa E_{+}-\frac{1}{2} \nu E_{+}+\frac{1}{2} \nu E_{-}\right),
\end{aligned}
$$

where $\kappa$ is the absorption coefficient, $v$ is the scattering coefficient, and $J$ is the total radiative energy (temperature and wavelength dependent) of the layer. The placement of the minus sign 
indicates that $E$. is defined as traveling in the positive $d x$ direction (this will be useful when considering plant canopies, where the origin is the top of the canopy and downward fluxes are positive). The assumption is that one half of the scattered radiation travels forward, and one half is scattered backward. However, this symmetry only occurs when the particles composing the medium are small compared to the wavelength of the radiation (Rayleigh 1914).

Silberstein (1927) applied the formulation to the case of a non-radiating medium illuminated by an external source. Thus $J=0$, and the divergence of the "primary beam" is described by

$$
\frac{d E_{s}}{d x}=-\left(\mu+s+s^{\prime}\right) E_{s},
$$

where $\mu . s$, and $s^{\prime}$ are the absorption, forward-scattering. and backward-scattering coefficients for the incident light, respectively. Ryde (1931) improved the formulation by noting that the scattering coefficients are generally different for incoming light than for the internally scattered diffuse light.

Duntley (1942) collected these improvements, and added the generalization that incident and diffuse radiation will have different absorption coefficients. Now the divergence of the diffuse fluxes for the "three-stream" formulation are:

$$
\begin{aligned}
& \frac{d E_{-}}{d x}=s E_{s}-\kappa E_{-}+\sigma E_{+}-\sigma^{\prime} E_{-} \\
& \frac{d E_{+}}{d x}=-\left(s^{\prime} E_{s}-\kappa E_{-}-\sigma^{\prime} E_{+}+\sigma E_{-}\right),
\end{aligned}
$$


where $\sigma$ and $\sigma^{\prime}$ are the scattering coefficients for downward (i.e., forward) and upward fluxes, respectively. Thus, all incident specular light not absorbed, $\left(s+s^{\prime}\right) E_{s}$, is assumed to be converted into a diffuse radiation source. This is essentially the formalism (Equations A.2 and A.3) used by Allen et al. (1970), who first applied the turbid medium concept to a plant canopy. They solved this equation for the outgoing diffuse flux $E_{+}(0)$ using the intuitive boundary conditions $E_{.}(0)=0, E_{s}(0)=1$. and $E_{+}\left(x_{L}\right)=\rho_{s} E_{-}\left(x_{L}\right)$. where $\rho_{s}$ is equal to the soil background reflectance. Further, the soil background is assumed to be Lambertian (reflecting all the incident radiation as upward diffuse flux). Allen et al. (1970) performed a quasivalidation of the model solution with data of NIR transmittance at three different heights in a corn canopy. In one case, they assumed that $\kappa=\mu=0$, and that $\sigma=\sigma^{\prime}$, but determined the other two parameters $\left(\sigma, s\right.$, and $\left.s^{\prime}\right)$ from a three-parameter fit to the data; in another, they used laboratory leaf measurements of absorption and scattering to estimate $\kappa$ and $\sigma$. They found $<4 \%$ error in both cases, and the measured parameters were within experimental error of their fitted counterparts.

The next improvement was made by Suits (1972), who considered a fourth flux associated with the outgoing radiance $L_{0}$ in an arbitrary direction of observation. This modification was a critical step toward simulating the BRDF. Suits (1972) accounted for anisotropic diffuse radiance by dividing the canopy conceptually into a number of layers, each with its own set of coefficients. The coefficients were decomposed into horizontal and vertical components which allowed for the coefficients to be expressed in terms of the optical properties of leaves. This obviously required as an input parameter the relative projected vertical and horizontal area of leaves in each layer. A unit thickness layer of the canopy (which is assumed Lambertian and so $E=\pi L$ ) contributes to the modeled directional radiance as follows: 


$$
d L_{o}=-\frac{1}{\pi}\left(u E_{+}+v E_{-}+w E_{s}-K E_{o}\right) d x
$$

where $u, v, w$ and $K$ are coefficients that depend on the relative horizontal and vertical projected leaf area of the layer, the angle of observation, and (in the case of $w$ ) the angle of incidence.

A more general and concise formalism was introduced by Verhoef (1984). It allowed for an arbitrary angular distribution of leaf inclination angles within a canopy layer by calculating iteratively the coefficients for Equations A.2-A.4 given a fixed inclination angle for a representative sample of angles in the distribution. The resulting radiative flow fields are superposed according to the relative abundance of the leaves at the given angle. Prior to this work, the geometry of source and sensor was accounted for (i.e., Suits 1972), but not the geometric character of the scattering elements, which is required for accurate representation of reflectance anisotropy. Further, Verhoef (1985) presented the model equations in a matrix form:

$$
\frac{d}{d t}(\mathbf{E})=\mathbf{M E}
$$

where $\mathbf{E}=\left(E_{s}, E_{.}, E_{+}, E_{\mathrm{o}}\right)^{\mathrm{T}}$ and $\mathbf{M}$ is a transformation given by 


$$
\mathbf{M}=\left(\begin{array}{cccc}
-k & 0 & 0 & 0 \\
s & a & \sigma & 0 \\
-s^{\prime} & -\sigma & -a & 0 \\
-w & -v & -u & K
\end{array}\right)
$$

where $k=\mu+s+s^{\prime}$ and $a=\kappa+\sigma^{\prime}$. The solution to this set of equations is straightforward. Let $\mathbf{Y}$ be a transformation of $\mathbf{E}$ such that $\mathbf{Y} \mathbf{M Y}^{-1}$ diagonalizes $\mathbf{M}$. Then Equation A.5 can be written as $d F / d x=\Lambda$, where $\Lambda$ is a diagonal matrix containing the eigenvalues of $M$. The standard form for the solution $\mathbf{F}$ can then be easily found. Applying the inverse transformation $E=Y^{-1} \mathbf{F}$ will then yield the final form of the fluxes. Application of boundary conditions can also follow the vector-matrix format. The major contribution of Verhoef $(1984,1985)$ was not the solution, but the definition of the coefficients $\mathbf{M}_{i j}$ in terms of the three primary angles (leaf inclination, solar zenith, and sensor zenith) and the leaf optical properties (reflectance and transmittance). So the set of model parameters $\mathbf{P}$, along with the sun-sensor configuration $\Theta$ (see Section 5.3.3) are mapped into the coefficients $\mathbf{M}_{i j}$ such that the reflectance is given by $R=R_{\mathrm{P}}(\lambda, \Theta)$. This allows field/laboratory-determined values of leaf optics and canopy architecture (LAI and leaf angle distribution) to be used in the simulation of canopy reflectance. Conversely, we can invert the model to obtain physically meaningful canopy parameters.

The version of the model used by Braswell et al. (1996) was modified to include nonphotosynthetic (NPV) scattering components. Qin (1993) demonstrated the importance of representing NPV in a canopy radiative transfer model, and showed that accurate bidirectional reflectance profiles can be obtained for mixed canopies. His method was to simply assume a linear superposition of the two sets of coefficients for a layer. This ignores interaction between the two components and assumes that an element of canopy thickness is described by a set of bulk canopy coefficients. Qin (1993), using a more complex model (based on geometric optics) 
than SAIL, found good agreement with bidirectional reflectance measurements of a wheat canopy, and a significant improvement over the original single component model. We performed the same test using the 2-component SAIL model (SAIL-2), with input parameters based on the Qin (1993) parameter set. Qualitatively, our results (unpublished) appear to agree about as well with the data in Qin (1993), though the degenerate case (zero NPV fraction) is somewhat different than their model.

Finally, the SAIL-2 model (Braswell et al. 1996) includes a parameterization of the hotspot effect. The model does not specifically deal with mechanisms that can give rise to the hotspot phenomenon, namely, self shading by leaves stems, trunks, and other plants. Thus, a modification of the flux $E_{\mathrm{o}}$ must be made. We used an implementation of a hot-spot parameterization for the SAIL model made by Kuusk (1991). Because the attenuation of the $E_{\mathrm{o}}$ flux (as a function of depth) is related to the probability of a line of sight observation of a layer at depth, the $K$ parameter (Equations A.4 and A.6) can be adjusted parametrically such that reflectance increases sharply where the observation and illumination angles are nearly equal (see Fig. 5.8). There is one parameter controlling the magnitude of the effect that can be related to the ratio of component (leaf) size to canopy height. 


\section{APPENDIX B \\ DETAILS OF THE INTEGRATED INVERSION ALGORITHM}

In this appendix, we will discuss some of the procedural details of the Braswell et al. (1996; Section 5.3) algorithm that were not fully presented in the article. Primarily these have to do with the initial analysis of the reflectance data leading to the choice of "cell" size and shape, the processing and application of ancillary information (e.g., field spectra), the gathering and filtering of data within a cell, the inversion method, and the calculation of albedo and $f A P A R$. Figure B.1 is a flow chart showing the steps in the process, which is essentially two-tiered. The upper tier, inside the box, is the main processing stream, and the lower tier is a supporting set of operations (both will be described below). What this diagram does not show is the considerable amount of exploratory data analysis required beforehand in order to verify the basic assumptions and requirements inherent in the method (related to exploiting the bidirectional information).

\section{B.1 Defining the neighborhood for pixel aggregation}

The basis of the approach is the creation of an "synthetic" bidirectional profile for unit of landscape (a cell) that is represented by an amalgam of pixels that are assumed to contain approximately the same vegetation mixture. Recall that each PAL pixel is $8 \times 8 \mathrm{~km}$ so the smallest unit is already mixed to some extent. Thus there are two competing requirements that need to be balanced: (1) the cell must be small enough so that the assumption of spatial continuity is not violated to a large degree (i.e., uncertainty in the true parameter set $\mathbf{P}$ 


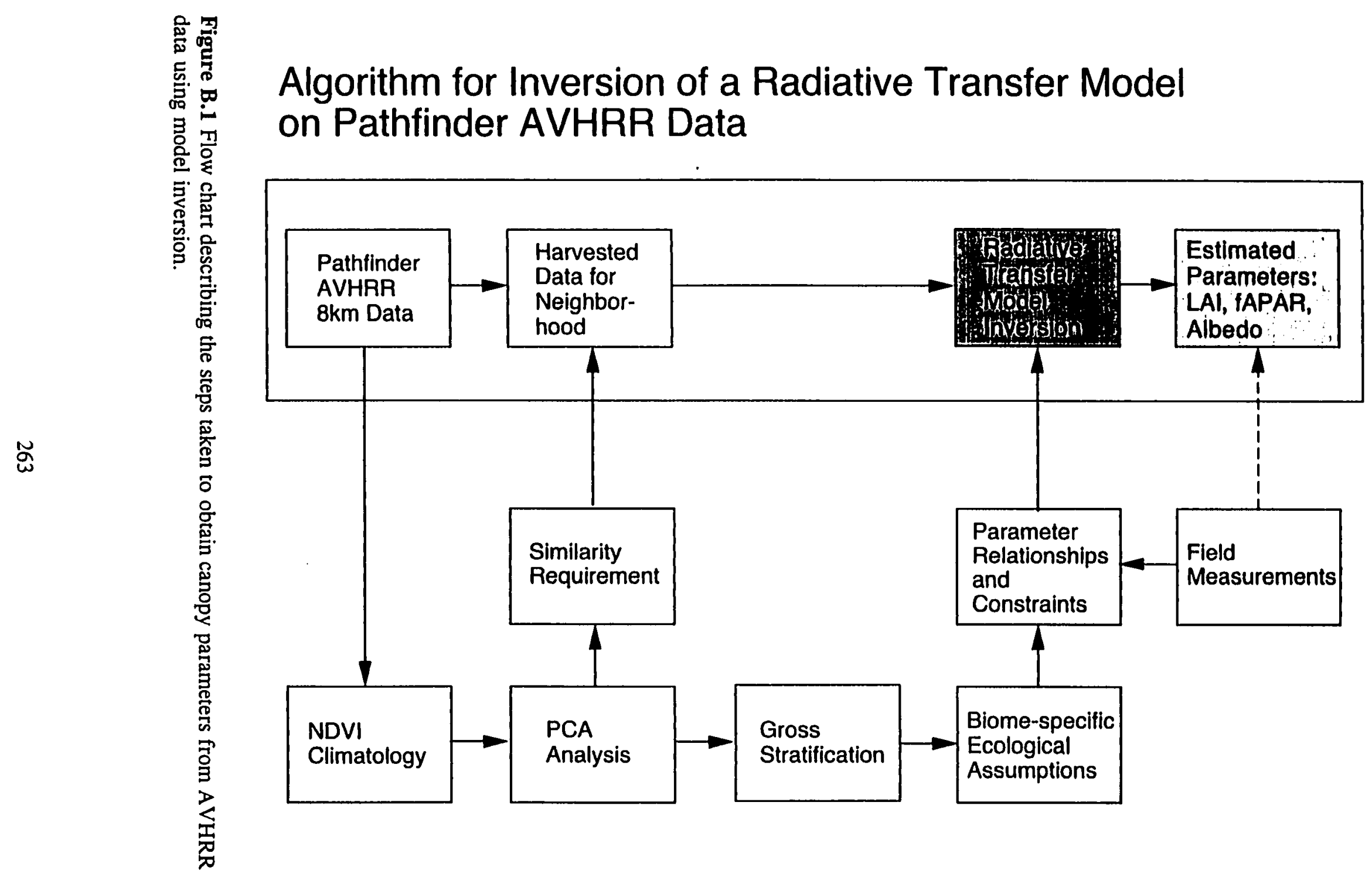


increases with cell size); and (2) the cell must be large enough to include significant variability in sun-sensor geometry (i.e., bidirectional information increases with cell size). The principal goal of the exploratory analysis mentioned above was to determine if a satisfactory compromise between these two considerations existed for the PAL data. Attempts to arrive at an objective, rule-based determination of cell size, shape, and orientation that could be applied on a continental scale all led either to some subjectivity (e.g., the specification of thresholds for variance) or an inconvenient degree of computational intensity. We settled on the uniform application of circular, $50 \mathrm{~km}$ radius cells based on a simple geostatistical analysis followed by a closer look at the variability of sun-sensor geometry for a few locations.

The work leading to the choice of cell specifications, though not part of the algorithm as shown in Fig. B.1, utilized two of the intermediate products: the NDVI climatology and the gross stratification (see Section 5.3.4). Figure B.2 shows the stratified data (Fig. 5.5) grouped into four distinct classes. We are particularly interested in the boundary between the Sahelian grassland/savanna region and the tropical evergreen forest region. We performed the semivariogram analysis on the monthly $\overline{\mathrm{NDVI}}$ (Equation 5.18) for all pixels in a class, using the formula (Cressie 1991):

$$
2 \hat{\gamma}(\mathbf{h})=\frac{1}{|N(\mathbf{h})|} \sum_{N(\mathbf{b})}\left(\overline{\operatorname{NDVI}}\left(\mathbf{s}_{i}\right)-\overline{\operatorname{NDVI}}\left(\mathbf{s}_{j}\right)\right)
$$

where $N(\mathbf{h})=\left\{(i, j): \mathbf{s}_{i}-\mathbf{s}_{j}=\mathbf{h}\right\}$. Further, because the data is gridded, we defined $\mathbf{h}$ such that $\hat{\gamma}$ was calculated for all possible spatial lags on a square grid $(|\mathbf{h}|<150 \mathrm{~km})$ in order to check for anisotropy. Our thinking at this point was that the cell shape used in the algorithm could be determined by the shape of the semivariogram surface for each class and for each month. 


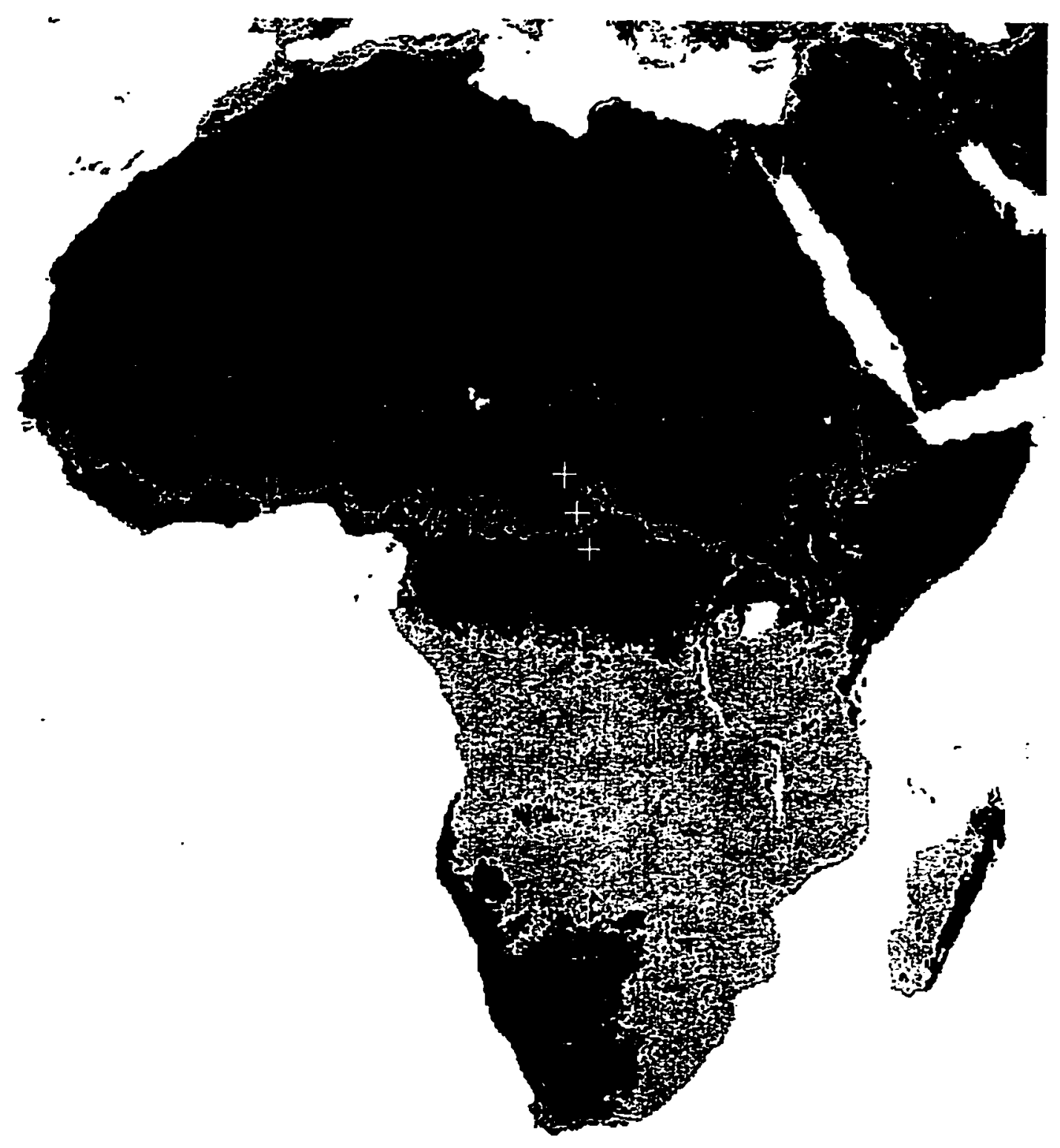

Figure B.2 A discrete classification of the first three principal components shown in Fig. 5.5. The area of focus for this study (white crosses) is divided into savanna/grassland and forest. 
Figure B. 3 shows a typical set of the $\overline{\text { NDVI }}$ semivariograms (for November). Though differences can be observed between classes related to different degrees of climate-driven gradients in vegetation productivity and (obviously) different degrees of heterogeneity associated with biome type, the anisotropy was oriented roughly along the north-south and eastwest axes. Thus we sought to define elliptical cells, whose size and semi-major axis lengths would be determined by length scales of constant semivariance in those directions. We based this analysis on the N-S/E-W semivariograms for two different months (May and November) and for the two classes that exist in our study region (class 2 =grassland/savanna: class $4=$ evergreen forest).

To evaluate the number of geometrically unique observations present in the data for a given cell size, we aggregated data for elliptical cells of varying size, centered on the crosses in Fig. B.2. The size was determined by the lag distance corresponding to an arbitrary semivariance. The polar plots in Fig. B.4 show the sampling of view zenith and relative azimuth for one of the cells, and for axes lengths corresponding to four values of $\hat{\gamma}(0.00 \mathrm{I}$, $0.0015,0.002$, and 0.0025 squared-NDVI units), while constraining the cells to be smaller than $10^{5} \mathrm{~km}$. We found that the number of unique geometries continued to increase with larger cells, but at length scales greater than $|\mathbf{h}| \approx 50 \mathrm{~km}$ (the range is generally $>100 \mathrm{~km}$ ) the differences were associated with pixel-to-pixel variability, which is considerably less than variability associated with the day/time of observation (there are a maximum of ten days worth of observations). At these relatively small spatial scales, there were typically more than two unique observations; in addition, the semivariograms were approximately isotropic and constant with respect to class and month. Thus, we opted for a circular cell of radius $50 \mathrm{~km}$. 
Nov. MM-NDVI Semivoriogram
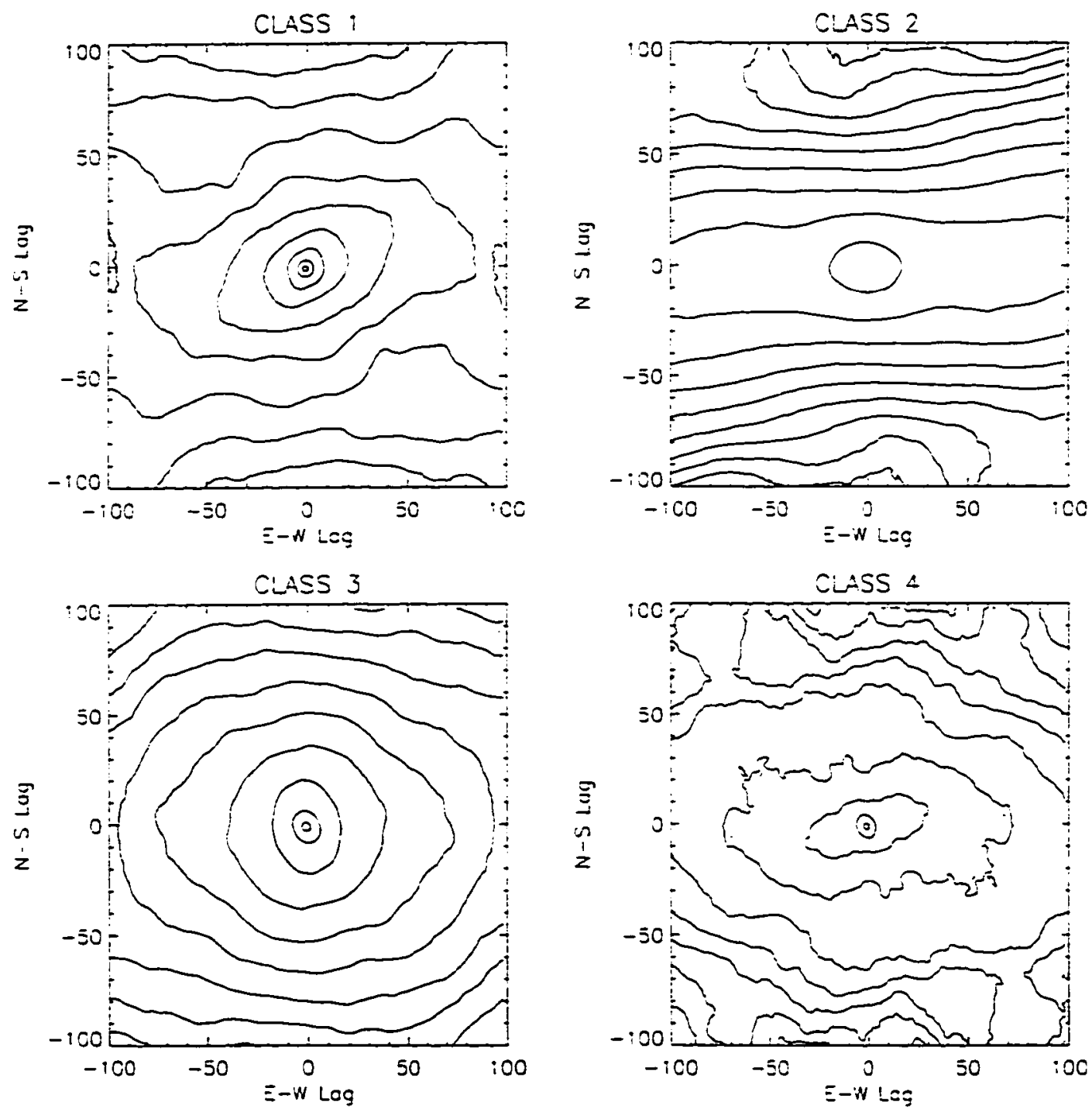

Figure B.3 Semivariogram contours of $\overline{\text { NDVI }}$ for the month of November for the four coarsely defined classes shown in (B.2). Class 1 is Desert, Class 2 is the northern seasonal savanna/grassiand, Class 3 is the southern savanna/grassland, and Class 4 is the evergreen forest. Classes 2 and 3 are kept separate so that monthly analyses would not be confounded by opposing seasonality. 


\section{Awakaba}
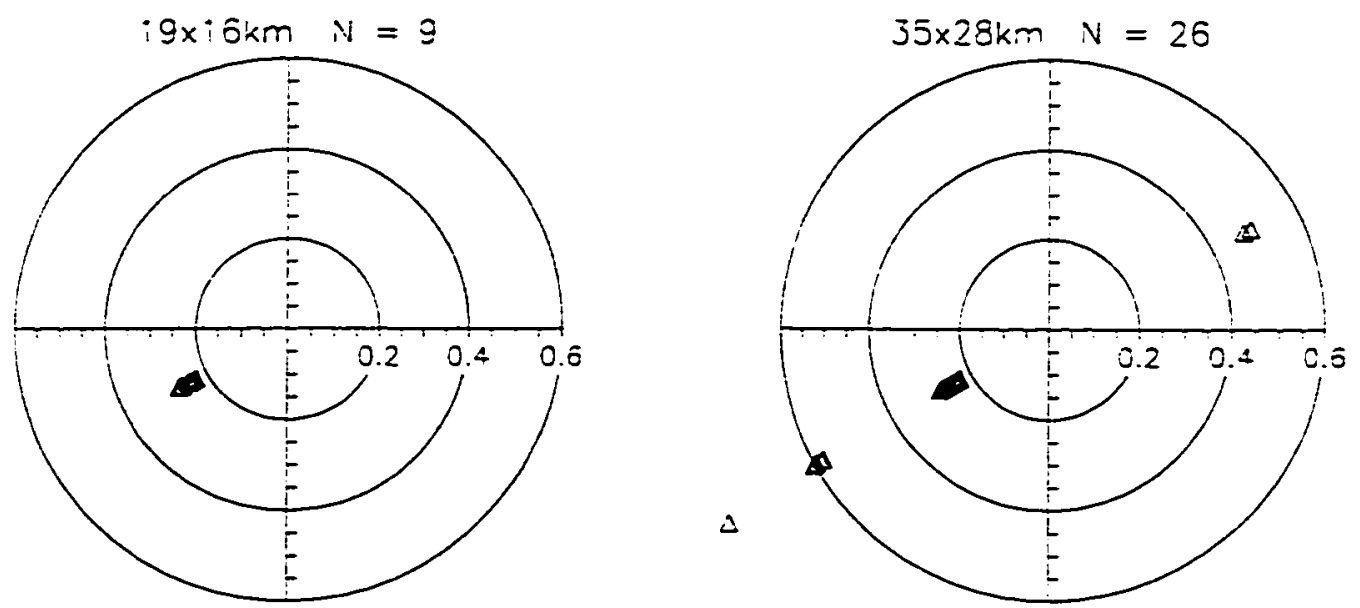

$\Delta$
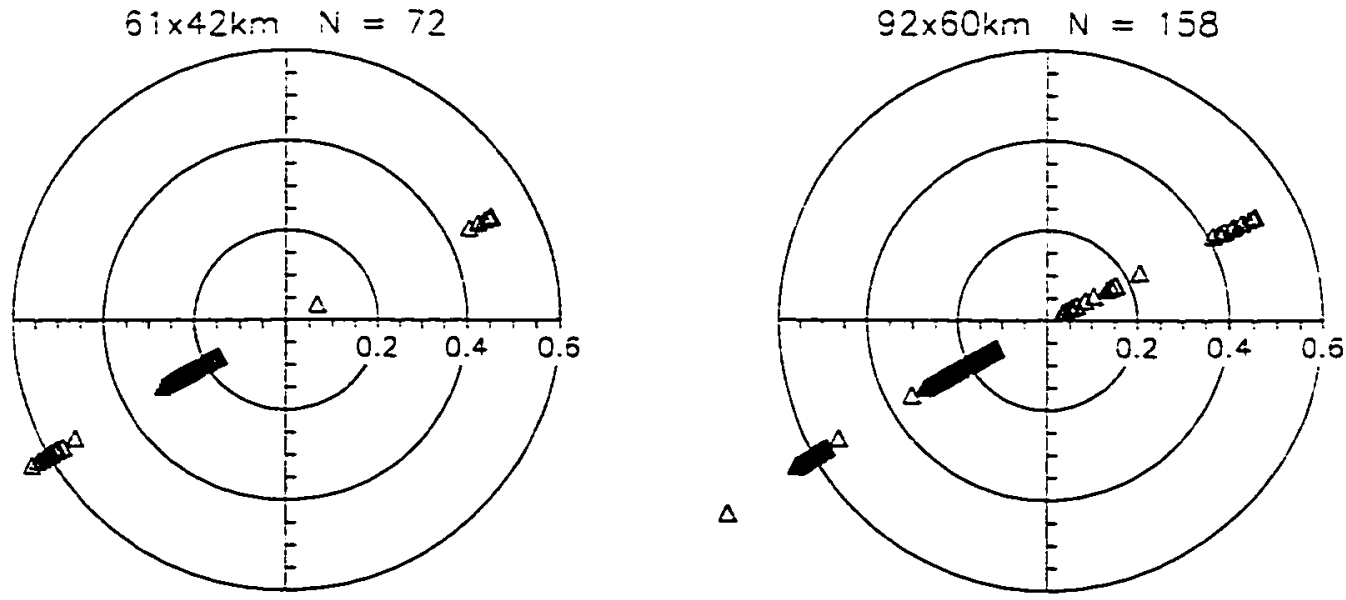

Figure B.4 Polar plots of the view zenith (rad) and azimuth for aggregated data with various cell sizes. The sun in all cases is at $0.7-0.8$ rad zenith, zero azimuth (cf. Fig. 5.8). Plot corresponds to the northernmost cross in Fig. B.2. 


\section{B.2 Incorporating field measurements}

We used high-spectral resolution data gathered for a variety of leaves and soil/litter backgrounds as a constraint on the inversion process. The data consisted of reflectance (for leaves and soil/litter) and transmittance (for leaves only) as a function of wavelength at $1.4 \mathrm{~nm}$ intervals measured by a field spectroradiometer. Two typical reflectance spectra are shown in Fig. 5.2. Each spectrum was converted to effective AVHRR visible and NIR band values, i.e., one point on Fig. 5.6 or 5.7, by a simple convolution:

$$
\hat{\rho}_{\Lambda}=\frac{\int_{\lambda} \rho(\lambda) \cdot I_{s}(\lambda) \cdot B_{\Lambda}(\lambda) d \lambda}{\int_{\lambda} I_{s}(\lambda) \cdot B_{\Lambda}(\lambda) d \lambda}
$$

where $B_{\mathrm{A}}$ is the sensor response curve, $\Lambda$ is either AVHRR band 1 or band 2 (see Fig. 5.2), $I_{s}$ is the solar spectrum, and the integral is over all wavelengths (but truncated by the finite width of $B_{\Lambda}$.

For the soil/litter spectra, we assumed the line in Fig. 5.7 to approximately describe the relationship between visible and NIR background reflectance, thus the two parameters $\left(P_{15}\right.$ and $P_{16}$ ) are reduced to one by the linear curve fit. Because the leaf optical parameters (and measurements) include transmittance as well as reflectance, a slightly more complicated procedure was used. We wished to reduce the four leaf optical parameters to one because they are correlated with one another (Fig. 5.6), and because it is necessary to have as few free parameters as possible. The principal component rotation of the leaf data is a linear transformation: 


$$
\begin{aligned}
& x_{1}=\alpha_{11} \rho_{v i s}{ }^{\prime}+\alpha_{12} \rho_{\mathrm{NIR}}+\alpha_{13} \tau_{\mathrm{vis}}+\alpha_{14} \tau_{\mathrm{NIR}} \\
& x_{2}=\alpha_{21} \rho_{\mathrm{vis}}+\alpha_{22} \rho_{\mathrm{NIR}}+\alpha_{23} \tau_{\mathrm{vis}}+\alpha_{24} \tau_{\mathrm{NIR}} \\
& x_{3}=\alpha_{31} \rho_{\mathrm{vis}}+\alpha_{32} \rho_{\mathrm{NIR}}+\alpha_{33} \tau_{\mathrm{vis}}+\alpha_{34} \tau_{\mathrm{NIR}} \\
& x_{4}=\alpha_{41} \rho_{\mathrm{vis}}{ }^{\prime}+\alpha_{42} \rho_{\mathrm{NIR}}+\alpha_{43} \tau_{\mathrm{vis}}+\alpha_{44} \tau_{\mathrm{NIR}}
\end{aligned}
$$

where the original data space is made up of the mean deviates of the four leaf optical parameters (e.g., $\rho_{\text {vis }}{ }^{\prime}=\rho_{\text {vis }}-\overline{\rho_{\text {vis }}}$ ) and $\mathbf{x}$ contains the transformed coordinates or principal components (PCs). The coefficients $\alpha_{i j}$ form a matrix $\mathbf{A}$ whose rows are the eigenvectors of the covariance matrix, i.e., the eigenvector corresponding to the first PC is $v_{1}=\left(\alpha_{11}, \alpha_{21}, \alpha_{31}\right.$, $\left.\alpha_{41}\right)^{\mathrm{T}}$. We wish to write the other variables in terms of $\rho_{\text {vis. }}$. This can be done using the first PC, which corresponds to a line oriented in the direction of maximum variance $(60 \%$ of the total variance in the data). First note that Equations B.2 can be inverted and that $\mathbf{A}^{-1}=\mathbf{A}^{\mathrm{T}}$. Thus, neglecting the second, third, and fourth PCs, we can substitute for $x_{1}$ :

$$
\begin{aligned}
& \rho_{\mathrm{NIR}}=\alpha_{12} x_{1}+\overline{\rho_{\mathrm{NIR}}}=\frac{\alpha_{12}}{\alpha_{11}} \rho_{\mathrm{vis}}+\left(\overline{\rho_{\mathrm{NIR}}}-\frac{\alpha_{12}}{\alpha_{11}} \overline{\rho_{\mathrm{vis}}}\right) \\
& \tau_{\mathrm{vis}}=\alpha_{13} x_{1}+\overline{\tau_{\mathrm{vis}}}=\frac{\alpha_{13}}{\alpha_{11}} \rho_{\mathrm{vis}}+\left(\overline{\tau_{\mathrm{vis}}}-\frac{\alpha_{13}}{\alpha_{11}} \overline{\rho_{\mathrm{vis}}}\right) \\
& \tau_{\mathrm{NIR}}=\alpha_{14} x_{1}+\overline{\tau_{\mathrm{NIR}}}=\frac{\alpha_{14}}{\alpha_{11}} \rho_{\mathrm{vis}}+\left(\overline{\tau_{\mathrm{NIR}}}-\frac{\alpha_{14}}{\alpha_{11}} \overline{\rho_{\mathrm{vis}}}\right),
\end{aligned}
$$

which, given the numerical values for the $\alpha_{1 j}$ values and the data means, are in slope-intercept form and shown as the dashed lines on Fig. 5.6. 


\section{B.3 Harvesting the data}

Prior to each inversion performed on a cell, a number of tests were applied to further enforce the requirement of spatial continuity, to reduce the effects of atmospheric contamination, and to eliminate pixels that were outliers for some other reason. Further, we aggregated pixels within cells that had nearly identical sun-sensor vectors. This regrouping substantially increased the computational efficiency of the inversion at essentially no cost to accuracy.

First, if one of the Pathfinder quality control flags was set, the pixel was thrown out. This condition could be due to a number of factors, including instrument malfunction, or an uncorrectable error in one of the processing steps (Agbu and James 1995). Second, if the Pathfinder cloud detection algorithm indicated any degree of cloudiness. the pixel was likewise rejected. Third, we applied the spatial continuity requirement using the mapped first three principal components of $\overline{\mathrm{NDVI}}\left(\mathrm{PC}_{i}\right.$; Section 5.3). The basic idea is to retain pixels that, on average, have a very similar time trajectory of NDVI. Thus, a strict requirement is that if a pixel has a $\mathrm{PC}_{i}$ value that is different by an amount $d$ from the mean, it is rejected. In other words, for the $k$ th pixel out of $N$ pixels in a cell, the criterion for acceptance is:

$$
\left|P C_{i k}-\frac{1}{N} \sum_{k=1}^{N} P C_{i k}\right|<d_{i}
$$

where $d_{i}$ is defined (arbitrarily) as $10 \%$ of the $3 \sigma$ width of the continental distribution of values of the $i$ th PC. Thus if any one pixel has a different $\operatorname{NDVI}(t)$ from the others (where "different" is defined relative to the possible range of values in the PC-transformed data), then it is not used. 


\section{B.4 The inversion technique}

Numerical inversion of the SAIL-2 model involved finding the minimum of the merit function $E^{2}\left(\mathbf{P}^{\prime} ; \Lambda, \Theta\right)$ (Equation 5.14), where $\mathbf{P}^{\prime}$ is a subset of the model parameters (the free parameters), and $(\Lambda, \Theta)$ is the set of wavelength bands and geometries for all the measurements of a target at a fixed time. Often, when inverting a set of equations using an optimization routine, a solution will be found that is a local minimum of the merit function, as opposed to a global minimum. The NaG E04JAF quasi-Newton algorithm attempts to verify that the minimum found is the desired solution, but it is still possible that a given starting point in parameter space will lead to a poor solution. We took two measures to ensure that either the most realistic and accurate solution will be found, or the routine will return with no solution. First, we applied simple bounds to the free parameters. This is equivalent to saying that $E^{2} \rightarrow \infty$ as $P_{i}^{\prime}$ approaches some critical value. See Table 5.1 for examples of parameter constraints. If the data and model will not allow for a physically realistic result, then the optimization routine returns with a "solution is outside domain" error condition. The second step was to perform the optimization a number of times with different (randomly initialized) starting points. The solution was retained that had the lowest sum-of-squares error.

\section{B.5 The calculation of albedo and fAPAR}

The calculation of albedo and $F A P A R$ required forward simulation of the model with the full solution set $\mathbf{P}$ (including the fixed and free parameters) determined in the inversion. We reported bihemispheric albedo (e.g., Ross 1975), which required integration by quadrature of Equation 5.17 using the forward-modeled reflectances. The wavelength-dependent parameters (e.g., leaf optics) were assumed to apply broadly over discrete intervals. In other words, the visible region parameters were used for $\lambda<0.7 \mu \mathrm{m}$, and the NIR parameters for $\lambda>0.7 \mu \mathrm{m}$. 
This is approximately true, but a more accurate estimate could be obtained by using a leaf model (Jacquemoud and Baret 1990), or an average spectral shape from the data.

Estimates of instantaneous fraction of absorbed photosynthetically active radiation $\left(f A P A R_{I}\right)$ required a further extension of the SAIL model. In order to calculate the absorbed radiation, one needs to know the upward and downward fluxes at the top and bottom of the canopy, i.e.,

$$
f A P A R_{l}=\frac{\left(E_{s}(0)+E_{-}(0)\right)-\left(E_{s}(I)+E_{+}(I)\right)}{E_{s}(0)+E_{-}(0)} .
$$

We added the calculation of the fluxes $E_{5}(1)$ and $E_{+}(1)$, which follow from the discussion of Verhoef (1984) under the assumption of a Lambertian background. Further, we reported values of "daily total fAPAR" (Equation 5.16; Goward and Huemmrich 1992). This requires forward model calculations at a number of solar zenith angles corresponding to a diurnal cycle. The incident solar flux was modeled as a function of latitude and time of day (e.g., Dingman 1994). 


\section{REFERENCES}

Aber, J.D., K.J. Nadelhoffer, P. Steudler, and J.M. Melillo (1989) Nitrogen saturation in Northern forest ecosystems. Bioscience 39:378-386.

Aber, J. D. and J. M. Melillo (1991) Terrestrial Ecosystems. Saunders College Publishing: Philadelphia.

Aber, J.D. and C.A. Federer (1992) A generalized, lumped-parameter model of photosynthesis, evaporation and net primary production in temperate and boreal forest ecosystems. Oecologia 92:463-474.

Aber, J.D., S.V. Ollinger, and C.T. Driscoll (In press) Modeling nitrogen saturation in forest ecosystems in response to land use and atmospheric deposition. Ecological Modeling.

Agbu, P.A. and M.E. James (1995) The NOAA/NASA Pathfinder AVHRR land data set user's manual. Goddard Distributed Active Archive Center, NASA, Goddard Space Flight Center, Greenbelt, MD.

Albritton, D.L., R.G. Derwent, I.S.A. Isaksen, M. Lal, D.J. Wuebbles, et al. (1995) Trace gas radiative forcing indices. In Climate Change 1994: Radiative Forcing of Climate Change, Cambridge University Press, pp. 211-231.

Allen, W.A., T.V. Gayle, A.J. Richardson (1970) Plant-canopy irradiance specified by the Duntley equations. Journal of the Optical Society of America 60, 372-376.

Alvez, D.S., L.G. Meira Filho, J.C.L. d'Alge, E.K. Mello, J.C. Moreira, J.S. de Madeiros (In press) The Amazonia information system. INPE Document.

Amthor, J.S. (1995) Terrestrial higher-plant response to increasing atmospheric $\left[\mathrm{CO}_{2}\right]$ in relation to the global carbon cycle. Global Change Biology 1:243-274.

Andres, L., W.A. Salas, D. Skole (1994) Fourier analysis of multi-temporal AVHRR data applied to a land cover classification. International Journal of Remote Sensing 15:11151121.

Asman, W.A.H. and H.A.V. Jaarsveld (1992) A variable-resolution transport model applied for $\mathrm{NH}_{\mathrm{x}}$ deposition in Europe. Atmospheric Environment 26A:445-464.

Asner, G.P., T.R. Seastedt, and A.R. Townsend (In press) The decoupling of terrestrial carbon and nitrogen cycles. Bioscience.

Asrar, G., M. Fuchs, E. T. Kanemasu, and J. H. Hatfield (1984) Estimating absorbed photosynthetic radiation and leaf area index from spectral reflectance in wheat. Agronomy Journal 76:300-306.

Bacastow, R. and C. D. Keeling (1973) Atmospheric carbon dioxide and radiocarbon in the natural carbon cycle: II. changes from AD. 1700 to 2070 as deduced from a geochemical model. In Carbon and the Biosphere, U. S. Atomic Energy Commission. NTIS document, CONF-720510.

Bakwin, P.S., S.C. Wofsy, and S.M. Fan (1990a) Measurement of reactive nitrogen oxides $\left(\mathrm{NO}_{\mathrm{y}}\right)$ within and above a tropical forest canopy in the wet season. Journal of Geophysical Research 95D: 16765.

Bakwin, P.S., S.C. Wofsy, S.M. Fan, M. Keller, S. Trumbore, and J.M. de Costa (1990b) Emission of nitric oxide from tropical forest soils and exchange of NO between the forest canopy and atmospheric boundary layers. Journal of Geophysical Research 95D:1675516764.

Baur, A. and A.L. Black (1983) Effect of tillage management on soil organic carbon and nitrogen. Farm Research 40:27-31. 
Bazzaz, F. A. (1990) The response of natural ecosystems to the rising global $\mathrm{CO}_{2}$ levels. Annual Review of Ecological Systems 21:167-196.

Bazzaz, F.A., and E.D. Fajer (1992) Plant life in a $\mathrm{CO}_{2}$-rich world. Scientific American 266:68-74.

Becker, F., H.-J. Bolle, and P.R. Rowntree (1988) The International Satellite Land-Surface Climatology Project, ISLSCP Rep. 10, 100 pp., COSPAR, Int. Assoc. of Meteorol. and Atmos. Phys., Berlin.

Benkovitz, C.M., J. Dignon, J. Pacyna, T. Acholtz, L. Tarrason, E.M. Voldner, and T.E. Graedel (In press) Global inventories of anthropogenic emissions of $\mathrm{SO}_{2}$ and $\mathrm{NO}_{\mathrm{x}}$. Journal of Geophysical Research.

Berendse, F., R. Aerts and R. Bobbink (1993) Atmospheric nitrogen deposition and its impact on terrestrial ecosystems. In Perspectives on plant competition (J.B. Grace and D. Tilman, Eds.), Academic Press, San Diego, CA. pp. 104-121.

Bird, M.I., J. Lloyd, and G.D. Farquhar (1994) Terrestrial carbon storage at the LGM. Nature 371:566.

Björkstrom, A. (1979) A model of $\mathrm{CO}_{2}$ interaction between atmosphere, oceans, and land biota. In SCOPE Report 13, Wiley \& Sons: Chichester.

Bloom, A.J., F.S. Chapin III, and H.A. Mooney (1985) Resource limitation in plants - an economic analogy, Annual Reviews of Ecological Systems 16:363-393.

Bolin, B., E. T. Degens, S. Kempe, and P. Ketner, eds. (1979) The Global Carbon Cycle. SCOPE 13, Wiley \& Sons: Chichester, England.

Bolin, B., ed. (1981) Carbon Cycle Modeling. SCOPE 16, Wiley \& Sons: Chichester, England.

Bolin, B. A., A. Björkstrom, K. Holmén, and B. Moore III (1983) The simultaneous use of tracers for ocean circulation studies. Tellus 35B:206-236.

Bolin, B. and I. Fung (1992) Report: the carbon cycle revisited. In Modeling the Earth System: Papers Arising from the 1990 OIES Global Change Institute, UCAR: Boulder, CO.

Bolker, B.M., S.W. Pacala, F.A. Bazzaz, C.D. Canham, and S.A. Levin (1995) Species diversity and ecosystem response to carbon dioxide fertilization: Conclusions from a temperate forest model. Global Change Biology 1:373-381.

Bonan, G.B. (1993) Physiological derivation of the observed relationship between net primary production and mean annual air temperature. Tellus 45B:397-408.

Bonan, G.B. (1995) Land-atmosphere $\mathrm{CO}_{2}$ exchange simulated by a land surface process model coupled to an atmospheric general circulation model. Journal of Geophysical Research 100:2817-2831.

Boring, L.R., W.T. Swank, J.B. Waide, and G.S. Henderson (1988) Sources, fates and impacts of nitrogen inputs to terrestrial ecosystems: review and synthesis. Biogeochemistry 6:119-159.

Bouman, B.A.M. (1992) Linking physical remote sensing models with crop growth simulation models, applied for sugar beet. International Joumal of Remote Sensing 13:2565-2581.

Brasseur, G.P., D.A. Hauglustaine, and S. Walters (In press) Chemical compounds in the remote Pacific troposphere: Comparison between MLOPEX measurements and chemical transport model calculations. Joumal of Geophysical Research.

Braswell, B.H., D.S. Schimel, D.S. Ojima, and P. Tans, Decadal-scale temperature anomalies and transient modeling of terrestrial ecosystem $\mathrm{CO}_{2}$ fluxes, paper presented at the International Union of Geodesy and Geophysics XXI General Assembly, Boulder CO, 2-14 July, 1995. 
Braswell, B.H., D.S. Schimel, J.L. Privette, B. Moore III. W.J. Emery, E.W. Sulzman, and A.T. Hudak (1996) Extracting ecological and biophysical information from AVHRR optical measurements: An integrated algorithm based on inverse modeling. Journal of Geophysical Research (Atmospheres) 101:23335-23348.

Braswell, B.H., D.S. Schimel, B. Moore III, and E. Linder (In preparation) Global terrestrial ecosystems respond to climate variability over multiple time scales.

Broecker, W.S., T. Takahashi, H.J. Simpson, and T.-H. Peng (1979) Fate of fossil fuel carbon dioxide and the global carbon budget. Science 206:409:418.

Broecker, W. S. and T.-H. Peng (1982) Tracers in the Sea. Lamont-Doherty Geological Observatory, Columbia University, Palisades, NY.

Broecker, W. S. (1991) Keeping global change honest. Global Biogeochemical Cycles 5:191192.

Broecker, W. S. and T.-H. Peng (1993) Evaluation of the ${ }^{13} \mathrm{C}$ constraint on the uptake of fossil fuel $\mathrm{CO}_{2}$ by the ocean. Global Biogeochemical Cycles 7:619-626.

Brown, O.W., J.W. Brown, and R.H. Evans (1985) Calibration of Advanced Very High Resolution Radiometer observations. Journal of Geophysical Research 90:1 1667-11677.

Burke, I.C., C.M. Yonker, W.J. Parton. C.V. Cole, K. Flach, and D.S. Schimel (1989) Texture, climate and cultivation effects on soil organic matter content in U.S. grassland soils. Soil Science Society of America Journal 53,800-805.

Burke, I.C., T.G.F. Kittel, W.K. Lauenroth, P. Snook, C.M. Yonker, and W.J. Parton (1991) Regional analysis of the central Great Plains. Bioscience 41:685-692.

Caldeira, K., and J.F. Kasting (1993) Insensitivity of global warming potentials to carbon dioxide emission scenarios. Nature 366, 251-253.

Canham, C.D. and S.W. Pacala (1995) Linking population dynamics and ecosystem processes. In Linking Species and Ecosystems, C.G. Jones, J.H. Lawton (Eds.) Chapman \& Hall. New York.

Chameides, W.L., P.S. Kasibhatla. J. Yienger, and H. Levy II (1994) Growth of continentalscale metro-agro-plexes, regional ozone pollution, and world food production. Science 264:74-77.

Chapin III, F.S., A.J. Bloom, C.B. Field, and R.H. Waring (1987) Plant responses to multiple environmental factors. Bioscience 37:39-57.

Chen, C.T.. and F.J. Millero (1979) Gradual increase of oceanic $\mathrm{CO}_{2}$. Nature 277:205-206.

Choudury, B.J. (1987) Relationships between vegetation indices, radiation absorption, and net photosynthesis evaluated by a sensitivity analysis. Remote Sensing of Environment 22:209233.

Ciais, P., P.P. Tans, J.W.C. White, M. Trolier, R.J. Francey, J.A. Berry, D.R. Randall, P.J. Sellers, J.G. Collatz, and D.S. Schimel (1995a) Partitioning of ocean and land uptake of $\mathrm{CO}_{2}$ as inferred by $\delta^{13} \mathrm{C}$ measurements from the NOAA Climate Monitoring and Diagnostics Laboratory Global Air Sampling Network. Journal of Geophysical Research 100D:5051-5070.

Ciais, P., P.P. Tans, M. Trolier, J.W.C. White, and R.J. Francey (1995b) A large Northern Hemisphere terrestrial $\mathrm{CO}_{2}$ sink indicated by the ${ }^{13} \mathrm{C} /{ }^{12} \mathrm{C}$ ratio of atmospheric $\mathrm{CO}_{2}$. Science 269:1098-1102.

Cihlar, J., D. Manak. and N. Voisin (1994) AVHRR bidirectional effects and compositing. Remote Sensing of Environment 48:77-88.

Cole, C.V., and R.D. Heil (1981) Phosphorus effects on terrestrial nitrogen cycling. In Terrestrial Nitrogen Cycles, F.E. Clark and T. Rosswall (Eds.) pp. 363-374, Swedish Natural Science Research Council, Stockholm. 
Cole. D.W., and M. Rapp (1981) Element cycling in forest ecosystems. In Dynamic Properties of Forest Ecosystems (D.E. Reichle, Ed.), Cambridge University Press, Cambridge. pp. $341-409$.

Comins, H.N. and R.E. McMurtrie (1993) Long-term response of nutrient limited forests to $\mathrm{CO}_{2}$ enrichment: equilibrium behavior of plant-soil models. Ecological Applications 3:666681.

Conway, T.J., P.P. Tans, L.S. Waterman, K.W. Thoning, D.R. Kitzis, K.A. Masarie, and N. Yang (1994) Evidence for interannual variability of the carbon cycle from the National Oceanic and Atmospheric Association / Climate Monitoring and Diagnostics Laboratory. Journal of Geophysical Research 99D:22831-22855.

Cramer, W., M. Claussen, and A.M. Solomon, An assessment of different climate change scenarios for the global redistribution of agricultural land, paper presented at the IGBP GAIM First Scientific Conference Proceedings, Garmisch-Partenkirchen, Germany, 24-29 Sept., 1995.

Cressie, N.A.C. (1991) Statistics for Spatial Data. John Wiley \& Sons, New York.

Crutzen, P.J. and P.H. Zimmerman (1991) The changing photochemistry of the troposphere. Tellus 43:136-151.

Dai A. and I.Y. Fung (1993) Can climate variability contribute to the 'missing' $\mathrm{CO}_{2}$ sink? Global Biogeochemical Cycles 7:599-609.

Davidson, E.A. (1991) Fluxes of nitrous oxide and nitric oxide from terrestrial ecosystems. In Microbial Production and Consumption of Greenhouse Gases: Methane, Nitrous Oxides. and Halomethanes (J.E. Rogers and W.E. Whitman, Eds.), American Society for Microbiology, Washington DC, pp. 219-236.

Davidson, E.A. and I.L. Ackerman (1993) Changes in soil carbon inventories following cultivation in previously untilled soils. Biogeochemistry 20:161-194.

DeFries, R.S., C.B. Field, I. Fung, C.O. Justice, S. Los, P.A. Matson, E. Matthews, H.A. Mooney, C.S. Potter, K. Prentice, P.J. Sellers, J.R.G. Townshend, C.J. Tucker, S.L. Ustin, and P.M. Vitousek (1995) Mapping the land surface for global atmospherebiosphere models: toward continuous distributions of vegetation's functional properties. Journal of Geophysical Research 100:20867-20882.

Dentener, F. and P. Crutzen (1993) Reaction of $\mathrm{N}_{2} \mathrm{O}_{5}$ on tropospheric aerosols: Impact on the global distributions of $\mathrm{NO}_{\mathrm{x}}, \mathrm{O}_{3}$, and $\mathrm{OH}$. Journal of Geophysical Research 98D:71497163.

Dentener, F. and P. Crutzen (1994) A three-dimensional model of the global ammonia cycle. Journal of Atmospheric Chemistry 19:331-370.

Díaz, S., J. P. Grime. J. Harris, and E. McPherson (1993) Evidence of a feedback mechanism limiting plant response to elevated carbon dioxide. Nature 364:616-617.

Dickinson, R.E. (1983) Land surface processes and climate-surface albedos and energy balance. Advances in Geophysics 25:305-353.

Diner, D.J., C.J. Bruegge, J.V. Martonchik, T.P. Ackerman, R. Davies, S. Gerstl, H.R. Gordon, P.J. Sellers, J. Clark, J.A. Daniels, E.D. Danielson, V.G. Duval, K.P. Klaasen, G.W. Lilienthal, D.I. Nakamoto, R.J. Pagano, and T.H. Reilly (1989) MISR: a multiangle imaging spectroradiometer for geophysical and climatological research from EOS. IEEE Transactions on Geoscience and Remote Sensing 27:200-211.

Dingman, S.L. (1994) Physical Hydrology. Macmillan Publishing, New York, 575 pp.

Dorman, J.L., and P.J. Sellers (1989) A global climatology of albedo, roughness length, and stomatal resistance for atmospheric general circulation models as represented by the Simple Biosphere Model (SiB). Journal of applied meteorology 28:833-855. 
Duntley, S.Q. (1942) The optical properties of diffusing materials. Journal of the Optical Society of America 32, 61-70.

Dutton, E.G. and J.R. Christy (1992) Solar radiative forcing at selected locations and evidence for global lower tropospheric cooling following the eruptions of El Chichon and Pinatubo. Geophysical Research Letters 19:2313-2316.

Eisele, K.A., D.S. Schimel, L.A. Kapustka, and W.J. Parton (1989) Effects of available P and $\mathrm{N}: \mathrm{P}$ ratios on non-symbiotic dinitrogen fixation in tallgrass prairie soils. Oecologia 79:471474.

Emanuel, W. R., G. G. Killough, W. M. Post, and H. H. Shugart (1984) Modelling terrestrial ecosystems in the global carbon cycle with shifts in carbon storage capacity by land use change. Ecology 65:970-983.

Enting, I.G., and J.V. Mansbridge (1989) Seasonal sources and sinks of atmospheric $\mathrm{CO}_{2}$ : direct inversion of filtered data. Tellus 41:111-126.

Enting, I.G., and J.V. Mansbridge (1991) Latitudinal distribution of sources and sinks of $\mathrm{CO}_{2}$ : results of an inversion study. Tellus 43:156-170.

Enting, I.G., T.M.L. Wigley, and M. Heimann (1994) Future emissions and concentrations of carbon dioxide: key ocean/atmosphere/land analyses. CSIRO Division of Atmospheric Research, technical document.

Esser, G. (1989) Global land-use changes from 1860 to 1980 and future projections to 2500. Ecological Modeling 44:307-316.

Esser, G., F. Mack, and U. Wittenberg (1993) High Resolution Biosphere Model Documentation. Institute for Plant Ecology, Justus-Liebig-University Giessen, Germany.

Farquhar, G.D., S. Von Caemmerer, J.A. Berry (1980) A biochemical model of photosynthetic $\mathrm{CO}_{2}$ assimilation in leaves of $\mathrm{C} 3$ species. Planta 149:78-90.

Farquhar, G.D., and T.D. Sharkey (1982) Stomatal conductance and photosynthesis. Annual Reviews of Plant Physiology 33:317-345.

Field, C.B. (1991) Ecological scaling of carbon gain to stress and resource availability. In Response of Plants to Multiple Stresses, H.A. Mooney, W.E. Winner and E.J. Pell (Eds.), pp. 35-65, Academic Press, San Diego.

Flasse, S.P. (1993) Extracting Quantitative Information from Satellite Data: Empirical and Physical Approaches. Ph.D. Thesis. Published by the Commission of the European Communities, Luxembourg. 199 pp.

Francey, R.J., P.P. Tans, C.E. Allison, I.G. Enting, J.W.C. White, and M. Trolier (1995) Changes in oceanic and terrestrial carbon uptake since 1982. Nature 373, 326-330.

Friedli, H., H. Lötscher, H. Oeschger, U. Siegenthaler, and B. Stauffer (1986) Ice core record of ${ }^{13} \mathrm{C} /{ }^{12} \mathrm{C}$ ratio of atmospheric $\mathrm{CO}_{2}$ in the past two centuries. Nature 324: 237-238.

Friedlingstein, P., I. Fung, E.A. Holland, J. John, G. Brasseur, D. Erickson, and D.S. Schimel (1995) On the contribution of $\mathrm{CO}_{2}$ fertilization to the missing biospheric sink. Global Biogeochemical Cycles 9:541-556.

Fung, I.Y., C.J. Tucker, and K.C. Prentice (1987) Application of advanced very high resolution radiometer to study atmosphere-biosphere exchange of $\mathrm{CO}_{2}$. Journal of Geophysical Research 92:2999-3015.

Fung, I., P. Friedlingstein, and A. Dai, Atmosphere-biosphere $\mathrm{CO}_{2}$ exchanges for the past 200 years: implications for a detection strategy, paper presented at the IGBP GAIM First Scientific Conference Proceedings, Garmisch-Partenkirchen, Germany, 24-29 Sept., 1995.

Gaffin, S.R., B.C. O'Neill, and M. Oppenheimer (1995) Comment on "The lifetime of excess atmospheric carbon dioxide" by Berrien Moore III and B.H. Braswell. Global Biogeochemical Cycles 9:167-174. 
Galloway, J.N., H. Levy II, and P.S. Kasibhatla (1994) Year 2020: consequences of population growth and development on deposition of oxidized nitrogen. Ambio 23:120-123.

Galloway, J.N., W.H. Schlesinger, H.L. Levy II, A. Michaels, and J.L. Schnoor (1995) Nitrogen fixation: anthropogenic enhancement - environmental response. Global Biogeochemical Cycles 9:235-252.

Garbutt, K., W. E. Williams, and F. A. Bazzaz (1990) Analysis of the differential response of five annuals to elevated $\mathrm{CO}_{2}$ during growth. Ecology 71:1 185-1194.

Gates, W. L., J. F. B. Mitchell, G. J. Boer, U. Cubasch, and V. P. Melesko (1992) Climate modelling, climate prediction, and model validation. In Climate Change 1992: The Supplementary Report to the IPCC Scientific Assessment, Cambridge University Press.

Gifford, R.M. (1994) The global carbon cycle: A viewpoint on the missing sink. Australian Journal of Plant Physiology 21:1-15.

Godbold, D.L., E. Fritz, and A. Huttermann (1988) Aluminum toxicity and forest decline. Proceedings of the National Academy of Sciences 85:388-3892.

Goel, N.S. and D.E. Streibel (1983) Inversion of vegetation canopy reflectance models for estimating agronomic variables: I. Problem definition and initial results using the Suits model. Remote Sensing of Environment 13:487-507.

Goel, N.S., and R.L. Thompson (1984) Inversion of vegetation canopy reflectance models for estimating agronomic variables, IV: Total inversion of the SAIL model. Remote Sensing of Environment 15:237-253.

Goulden, M.L., J.W. Munger, S.-M. Fan, B.C. Daube, and S.C. Wofsy (1996) Exchange of carbon dioxide by a deciduous forest: Response to interannual climate variability. Science 271:1576-1578.

Goward, S.N., and K.F. Huemmrich (1992) Vegetation canopy PAR absorptance and the normalized difference vegetation index: an assessment using the SAIL model. Remote Sensing of Environment 39:119-140.

Gundersen, P. (1991) Nitrogen deposition and the forest nitrogen cycle: role of denitrification. Forest Ecology and Management 44:15-28.

Haas, T.C. (1990) Kriging and automated variogram modeling within a moving window. Atmospheric Environment 24A:1759-1769.

Hall, F., Y.E. Shimabukuro, and K.F. Huemmrich (1995) Remote sensing of forest biophysical structure using mixture decomposition and geometric reflectance models. Ecological Applications 5:993-1013.

Hansen, J., A. Lacis, R. Ruedy, and M. Sato (1992) Potential climate impact of Mount Pinatubo eruption. Geophysical Research Letters 19:215-218.

Hedin, L.O., J.J. Armesto, and A.H. Johnson (1995) Patterns of nutrient loss from unpolluted, old-growth temperate forests: Evaluation of biogeochemical theory. Ecology 76:493-509.

Henderson-Sellers, A., K. McGuffie, and C. Gross (1995) Sensitivity of global climate model simulations to increased stomatal resistance and $\mathrm{CO}_{2}$ increases. Journal of Climate 8:1738-.

Holben, B.N. (1986) Characteristics of maximum-value composite images from temporal AVHRR data. International Journal of Remote Sensing 7:1417-1434.

Holland, E.A., B. Hungate, F. Chapin, A.R. Townsend, and C. Field. Foliar, root, and microbial contributions to ecosystem respiration under elevated $\mathrm{CO}_{2}$ using ${ }^{13} \mathrm{C}$ techniques. American Geophysical Union, Fall Meeting, San Francisco, CA, December 5-9, 1994.

Holland, E.A., A.R. Townsend, and P.M. Vitousek (1995) Variability and temperature regulation of $\mathrm{CO}_{2}$ fluxes and $\mathrm{N}$ mineralization from five Hawaiian soils. Global Change Biology 1:115-124. 
Holland, E.A., B.H. Braswell, J.-F. Lamarque, A.R. Townsend, J. Sulzman, J.-F. Müller, F. Dentener, G. Brasseur, H. Levy II, J.E. Penner, G.-J. Roelofs (In press) The spatial distribution of atmospheric nitrogen deposition and its impact on carbon uptake by terrestrial ecosystems. Journal of Geographic Research (Atmospheres).

Houghton, R.A., R.D. Boone, J.M. Melillo, C.A. Palm, G.M. Woodwell, N. Meyers, B. Moore, and D.L. Skole (1983a) Net flux of carbon dioxide from tropical forests in 1980. Nature 316:617-620.

Houghton, R.A., J.E. Hobbie, J.M. Melillo, B. Moore, B.J. Peterson, G.R. Shaver, and G.M. Woodwell (1983b) Changes in the carbon content of terrestrial biota and soils between 1860 and 1980: A net release of $\mathrm{CO}_{2}$ to the atmosphere. Ecological Monographs 53:235262.

Houghton, R.A., W.H. Schlesinger, S. Brown, and J.F. Richards (1985) Carbon dioxide exchange between the atmosphere and terrestrial ecosystems. In Atmospheric Carbon Dioxide and the Global Carbon Cycle (J.R. Trabalka, Ed.), DOE/ER-0239, US Dept. of Energy, Washington, DC.

Houghton, R.A., and D.L. Skole (1990) Changes in the global carbon cycle between 1700 and 1985. In The Earth Transformed by Human Action. Cambridge University Press: New York.

Houghton, R.A. (1991) Tropical deforestation and atmospheric carbon dioxide. Climatic Change 19:99-118.

Houghton, R.A., D.L. Skole, and D.S. Lefkowitz (1991) Changes in the Landscape of Latin America between 1850 and 1980: II. A net release of $\mathrm{CO}_{2}$ to the atmosphere. Forest Ecology and Management 38:173-199.

Houghton, R.A. (1993a) Is carbon accumulating in the Northern temperate zone? Global Biogeochemical Cycles 7:611-617.

Houghton, R.A. (1993b) Emissions of carbon from land-use change. The 1993 Global Change Institute: The Carbon Cycle. Snowmass, CO, July, 1993.

Houghton, R.A. (1995) Land-use change and the carbon cycle. Global Change Biology 1:275287.

Howarth, R.W., G. Billen, D. Swaney, A.R. Townsend, N. Jaworski, K. Lajtha, J. Downing, R. Elmgren, N. Caraco, T. Jordan, F. Berendse, J. Freney, V. Kudeyarov, P. Murdoch, and Shu Zhao-liang. (In press) Regional nitrogen budgets and riverine N \& P fluxes for the drainages to the North Atlantic Ocean: natural and human influences. Biogeochemistry.

Hudson, R.J.M., S.A. Gherini, R.A. Goldstein (1994) Modeling the global carbon cycle: Nitrogen fertilization of the terrestrial biosphere and the "missing" $\mathrm{CO}_{2}$ sink. Global Biogeochemical Cycles 8:307-333.

Huete, A. (1988) A soil adjusted vegetation index (SAVI). Remote Sensing of Environment 25:295-309.

Ingestadt, T., and A.B. Lund (1986) Theory and technique for steady state mineral nutrition and growth of plants. Scandinavian Journal of Forest Research 1:439-453.

IPCC (1995) Climate Change 1994: Radiative Forcing of Climate Change and An Evaluation of the IPCC IS92 Emission Scenarios, J.T. Houghton, L.G. Meira Filho, J. Bruce, H. Lee, B.A. Callander, E. Haites, N. Harris and K. Maskell (Eds.), Cambridge University Press, Cambridge, UK.

Intergovernmental Panel on Climate Change

Isaksen, I. S. A., J. Ramaswamy, H. Rodhe, and T. M. L. Wigley (1992) Radiative forcing of climate. In Climate Change 1992: The Supplementary Report to the IPCC Scientific Assessment, Cambridge University Press, pp. 47-68. 
Jacob, D.J. and P.S. Bakwin (1991) Cycling of $\mathrm{NO}_{\mathrm{x}}$ in tropical forest canopies. In Microbial Production and Consumption of Greenhouse Gases: Methane, Nitrous Oxides, and Halomethanes (J.E. Rogers and W.E. Whitman, Eds.), American Society for Microbiology, Washington DC, pp. 237-254.

Jacquemoud, S. and F. Baret (1990) PROSPECT: A model of leaf optical properties spectra. Remote Sensing of Environment 34:75-91.

Johnson, D.W., M.S. Cresser, S.I. Nilsson, J. Turner, B. Ulrich, D. Binkley, and D.W. Cole (1991) Soil changes in forest ecosystems: evidence for and probable causes. Proceedings of the Royal Society of Edinburgh, (Series B) 97:81-116.

Johnson, D.W. (1992) Nitrogen retention in forest soils. Journal of Environmental Quality 21:1-12.

Kasibhatla, P.S., H. Levy II, W.J. Moxim, and W.L. Chameides (1991) The relative impact of stratospheric photochemical production on tropospheric $\mathrm{NO}_{\mathrm{y}}$ levels: A model study. Journal of Geophysical Research 96D:18631-18646.

Kasibhatla, P.S., H. Levy II, and W.J. Moxim (1993) Global $\mathrm{NO}_{x}, \mathrm{HNO}_{3}$, PAN, and $\mathrm{NO}_{\mathrm{y}}$ distributions from fossil fuel combustion emissions: A model study. Journal of Geophysical Research 98D:7165-7180.

Kaufman, Y.J. (1989) The atmospheric effect on remote sensing and its correction. In Theory and Applications of Optical Remote Sensing, G. Asrar (Ed.) John Wiley \& Sons, New York.

Kaufmann, A., and M.M. Gupta (1985) Introduction to Fuzzy Arithmetic. Van Nostrand Reinhold, New York.

Kauppi, P.E., K. Mielikainen, and K. Kuusela (1992) Biomass and carbon budget of European forests, 1971-1990. Science 256:70-74.

Keeling, C.D., R.B. Bacastow, A.E. Bainbridge, C.A. Ekdahl, and P.R. Guenther (1976) Atmospheric carbon dioxide variations at Mauna Loa observatory. Tellus 28, 538-551.

Keeling, C. D. (1986) Atmospheric $\mathrm{CO}_{2}$ concentrations - Mauna Loa Observatory, Hawaii 1958-1986. NDP-001/R1. Carbon Dioxide Information Analysis Center. Oak Ridge National Laboratory, Oak Ridge, TN.

Keeling, C.D., R.B. Bacastow, A.F. Carter, S.C. Piper, T.P. Whorf, M. Heimann, W.G. Mook, and H. Roeloffzen (1989a) A three-dimensional model of atmospheric CO2 transport based on observed winds: 1. Analysis of observational data. In Aspects of Climate Variability in the Pacific and the Western Americas, D.H. Peterson (ed.). American Geophysical Union.

Keeling, C. D., S. C. Piper, and M. Heimann (1989b) A three-dimensional model of atmospheric $\mathrm{CO}_{2}$ transport based on observed winds: 4. Mean annual gradients and interannual variability. In Aspects of Climate Variability in the Pacific and Western Americas, Geophysical Monograph 55, AGU: Washington, DC.

Keeling, C.D. (1994) Global historical $\mathrm{CO}_{2}$ emissions. In Trends '93: A Compendium of Data on Global Change (T.A. Boden et al. Eds.), CDIAC, Oak Ridge National Laboratory, Oak Ridge TN. pp. 501-504.

Keeling, C.D., T.P. Whorf, M. Wahlen, and J. van der Plicht (1995) Interannual extremes in the rate of rise of atmospheric carbon dioxide since 1980. Nature 375:666-670.

Keeling, C.D., J.F.S. Chin, and T.P. Whorf (1996) Increased activity of northern vegetation inferred from atmospheric $\mathrm{CO}_{2}$ measurements. Nature 382:146-149.

Keeling, R.F., S.C. Piper, and M. Heimann (1996) Global and hemispheric $\mathrm{CO}_{2}$ sinks deduced from changes in atmospheric $\mathrm{O}_{2}$ concentration. Nature 381:218-221. 
Khalil, M.A.K., and R.A. Rasmussen (1983) Increase and seasonal cycles of nitrous oxide in the earth's atmosphere. Tellus 35B:161-169.

Kidwell, K.B. (1991) NOAA Polar Orbiter Data User's Guide. NCDC/SDSD, National Climatic Data Center, Washington, DC.

Kohlmaier, G.H. A. Janacek, M. Plochl (1988) Modeling response of vegetation to both excess $\mathrm{CO}_{2}$ and airborne nitrogen compounds within a global carbon cycle model. In Advances in Environmental Modeling (A. Marani, Ed.), Elsevier, New York, pp. 207-234.

Kuusk, A. (1991) The hot spot effect in plant canopy reflectance. In Photon-Vegetation Interactions: Applications in Optical Remote Sensing and Plant Ecology (R.B. Myneni and J. Ross, Eds.), Springer-Verlag, Berlin, pp. 9-44.

Lamarque, J.-F., G. Brasseur, P.G. Hess, and J.-F. Müller (In press) Three-dimensional study of the relative contributions of the different nitrogen sources in the troposphere. Journal of Geophysical Research.

Lashof, D. A. and D. R. Ahuja (1990) Relative contributions of greenhouse gas emissions to global warming. Nature 344:529-531.

Leith, H. (1975) Primary production of the major vegetation units of the world. In Primary Productivity of the Biosphere, H. Leith and R.H. Whittaker (Eds.), pp. 204-215, SpringerVerlag, New York.

Lenschow, D.H. (1995) Micrometeorological techniques for measuring biosphere-atmosphere trace gas exchange. In Biogenic Trace Gases: Measuring Emissions from Soil and Water, P.A. Matson and R.C. Harriss (Eds.), pp. 126-163, Blackwell Science, Cambridge, MA, USA.

Levy II, H. and W.J. Moxim (1989a) Influence of long-range transport of combustion emissions on the chemical variability of the background atmosphere. Nature 338:326-328.

Levy II, H. and W.J. Moxim (1989b) Simulated global distribution and deposition of reactive nitrogen emitted by fossil fuel combustion. Tellus 41B:256-271.

Levy II, H., P.S. Kasibhatla, W.J. Moxim, and J.A. Logan (1991) The global impact of biomass burning on tropospheric reactive nitrogen. In Proceedings of the Chapman Conference on Biomass Burning (J. Levine, Ed.), MIT Press, Cambridge MA, pp. 263269.

Levy II, H., W.J. Moxim, and P.S. Kasibhatla (In press a) Global 3-dimensional timedependent lightning source of tropospheric $\mathrm{NO}_{\mathbf{x}}$. Journal of Geophysical Research.

Levy II, H., W.J. Moxim, and P.S. Kasibhatla (In press b) Tropospheric $\mathrm{NO}_{\mathrm{x}}$ : Its sources and distribution. Journal of Geophysical Research.

Likens, G.E., F.H. Bormann, and N.M. Johnson (1981) Interactions between major biogeochemical cycles in terrestrial ecosystems. In Some Perspectives of the Major Biogeochemical Cycles (G.E. Likens, Ed.), Wiley, New York, pp. 93-112.

Logan, J.A., M.J. Prather, S.C. Wofsy, and M.B. McElroy (1981) Tropospheric chemistry: a global perspective. Journal of Geophysical Research 86:7210-7254.

Logan, J.A. (1983) Nitrogen oxides in the troposphere: Global and regional budgets. Journal of Geophysical Research 88D:10785-10807.

Maier-Reimer, E. and K. Hasselmann (1987) Transport and storage of $\mathrm{CO}_{2}$ in the ocean - an inorganic ocean-circulation carbon cycle model. Climate Dynamics 2:63-90.

Maier-Reimer, E. (1993) Geochemical cycles in an ocean general circulation model. Preindustrial tracer distributions. Global Biogeochemical cycles 7:645-677.

Maisongrande, P., A. Ruimy, G. Dedieu, and B. Saugier (1995) Monitoring seasonal and interannual variations of gross primary productivity, net primary productivity and net ecosystem productivity using a diagnostic model and remotely-sensed data. Tellus 47B: 178 190. 
Mälkönen, E. (1990) Estimation of nitrogen saturation on the basis of long-term fertilization experiments. Plant and Soil 128:75-82.

Marland, G.R., J. Andres, and T.A. Boden (1994) Global, regional and national $\mathrm{CO}_{2}$ emissions. In Trends '93: A Compendium of Data on Global Change (T.A. Boden et al. Eds.), CDIAC, Oak Ridge National Laboratory, Oak Ridge TN. pp. 501-504.

Matson, P.A., and P.M. Vitousek (1987) Cross-system comparisons of soil nitrogen transformations and nitrous oxide flux in tropical forest ecosystems. Global Biogeochemical Cycles 1:163-170.

Matthews, E. (1983) Global vegetation and land-use: new high-resolution databases for climate studies. Journal of Climate and Applied Meteorology 22:474-487.

McGuire, A.D., J.M. Melillo, L.A. Joyce, D.W. Kicklighter, A.L. Grace, B. Moore III, and C.J. Vorosmarty (1992) Interactions between carbon and nitrogen dynamics in estimating net primary productivity for potential vegetation in North America. Global Biogeochemical Cycles 6:101-124.

McLaughlin, S.B. and D.J. Downing (1995) Interactive effects of ambient ozone and climate measured on growth of mature forest trees. Nature 374:252-254.

Melillo, J.M., R.J. Naiman, J.D. Aber, and A.E. Linkins (1984) Factors controlling mass loss and nitrogen dynamics of plant litter decaying in northern streams. Bulletin of Marine Science 35:341-356.

Melillo, J.M., J.R. Fruci, R.A. Houghton, B. Moore, and D.L. Skole (1988) Land-use change in the Soviet Union between 1850 and 1980: causes of a net release of $\mathrm{CO}_{2}$ to the atmosphere. Tellus 40B:116-128.

Melillo, J.M., A.D. McGuire, D.W. Kicklighter, B. Moore III, C.J. Vorosmarty, and A.L. Schloss (1993) Global climate change and terrestrial net primary production. Nature 363:234-240.

Mitchell, J. F. B., S. Manabe, V. Meleshko, and T. Tokioka (1990) Equilibrium Climate Change - and its Implications for the Future. In Climate Change: The IPCC Scientific Assessment, Cambridge University Press.

Moore, B., R.D. Boone, J.E. Hobbie, R.A. Houghton, J.M. Melillo, B.J. Peterson, G.R. Shaver, C.J. Vorosmarty, and G.M. Woodwell (1981) A Simple model for analysis for the role of terrestrial ecosystems $\mathrm{n}$ the global carbon budget. In Carbon Cycle Modeling, B. Bolin, Ed., pp. 365-386.

Moore, B. (1985) The oceanic sink for excess atmospheric carbon dioxide. In Wastes in the Ocean, vol. 4: Energy Wastes in the Ocean, Wiley \& Sons: Chichester.

Moore, B. (1992) Four simple ocean carbon models. In Modeling the Earth System, UCAR/Office of Interdisciplinary Studies: Boulder, CO.

Moore, B. and B.H. Braswell (1994) The lifetime of excess atmospheric carbon dioxide. Global Biogeochemical Cycles 8:23-38.

Müller, J.-F. (1992) Geographical distribution and seasonal variation of surface emissions and deposition velocities of trace gases. Journal of Geophysical Research 97D:3787-3804.

Müller, J.-F. and G. Brasseur (1995) IMAGES: A three-dimensional chemical transport model of the global troposphere. Journal of Geophysical Research 100D:16445-16490.

Myneni, R.B., G. Asrar and F.G. Hall (1992a) A three dimensional radiative transfer model for optical remote sensing of vegetated land surfaces. Remote Sensing of Environment 41:105-121.

Myneni, R. B., B. D. Ganapol, and G. Asrar (1992b) Remote sensing of vegetation canopy photosynthetic and stomatal conductance efficiencies. Remote Sensing of Environment 42:217-238. 
Myneni, R. B., F. G. Hall, P. J. Sellers, and A. L. Marshak (1995), The meaning of spectral vegetation indices, Transactions of Geoscience and Remote Sensing, 33:481-486.

Myneni, R.B., S.O. Los, and C.J. Tucker (1996) Satellite-based identification of linked vegetation index and sea surface temperature anomaly areas from 1982-1990 for Africa, Australia and South America. Geophysical Research Letters 7:729-732.

Nadelhoffer, K.J., J.D. Aber, and J.M. Melillo (1985) Fine root production in relation to total net primary production along a nitrogen availability gradient in temperare forests: a new hypothesis. Ecology 66:1377-1390.

NADP/NTN, National Atmospheric Deposition Program / National Trends Network Coordination Office, Natural Resource and Ecology Laboratory, Colorado State University, Fort Collins CO.

National Aeronautics and Space Administration (1984) Earth Observing System: Science and Mission Requirements, Working Group Report Volume I, Part I, NASA Technical Memorandum 86129, Goddard Space Flight Center, Greenbelt, MD.

Neftel, A., E. Moor, H. Oeschger, and B. Stauffer (1985) Evidence from polar ice cores for the increase in atmospheric $\mathrm{CO}_{2}$ in the past two centuries. Nature 315:45-47.

Nevison, C. (1994) A global model for nitrous oxide production in terrestrial ecosystems. Ph.D. thesis, Stanford University.

Nevison, C.D., G. Esser, and E.A. Holland (1996) A global model for changing $\mathrm{N}_{2} \mathrm{O}$ emissions from natural and perturbed soils. Climatic Change 32:327-378.

Nobel, P.S. (1991) Physicochemical and Environmental Plant Physiology, 635 pp., Academic Press, San Diego, California.

Oechel, W.C., S. Cowles, N. Grulke, S.J. Hastings, B. Lawrence, T. Prudhomme, G. Reichers, B. Strain, D. Tissue, and G. Vourlitis (1994) Transient nature of $\mathrm{CO}_{2}$ fertilization in Arctic tundra. Nature 371:500-503.

Oeschger, H., U. Siegenthaler, U. Schotterer, and A. Gugelmann (1975) A box-diffusion model to study the carbon dioxide exchange in nature. Tellus 27:168-192.

Ojima, D.S., D.S. Schimel, W.J. Parton, and C.E. Owensby (1994) Long- and short-term effects of fire on nitrogen cycling in tallgrass prairie. Biogeochemistry 24:67-84.

Olsson, L. and L. Eklund (1994) Fourier series for analysis of temporal sequences of satellite sensor imagery. International Journal of Remote Sensing 15:3735-3741.

O'Neill, B.C., S.R. Gaffin, F.N. Tubiello, and M. Oppenheimer (1994) Reservoir timescales for anthropogenic $\mathrm{CO}_{2}$ in the atmosphere. Tellus 46B:378-389.

Parton, W.J., D.S. Schimel, C.V. Cole, and D.S. Ojima (1987) Analysis of factors controlling soil organic matter levels in Great Plains grassiands. Soil Science Society of America 51:1173-1179.

Parton, W.J., J.W.B. Stewart, and C.V. Cole (1988) Dynamics of C, N, P, and S in grassland soils: A model. Biogeochemistry 5:109-131.

Parton, W.J., J.M.O. Scurlock, D.S. Ojima, T.G. Gilmanov, R.J. Scholes, D.S. Schimel, T. Kirchner, T. Seastedt, E. Garcia Moya, Apinan Kamnalrut, and J.I. Kinyamario (1993) Observations and modeling of biomass and soil organic matter dynamics for the grassland biome worldwide. Global Biogeochemical Cycles 7:785-809.

Parton, W.J., D.S. Ojima, C.V. Cole, and D.S. Schimel (1994) A general model for soil organic matter dynamics: Sensitivity to litter chemistry, texture and management. In Quantitative Modeling of Soil Forming Processes, R.B. Bryant and R.W. Arnold (Eds.), pp. 147-167, Soil Science Society of America, Madison, WI. 
Parton, W.J., J.M.O. Scurlock, D.S. Ojima, D.S. Schimel, D.O. Hall, and SCOPEGRAM Group Members (1995) Impact of climate change on grassland production and soil carbon worldwide. Global Change Biology 1:13-22.

Pastor, J., and W.M. Post (1993) Linear regressions do not predict the transient responses of eastern North American forests to $\mathrm{CO}_{2}$-induced climate change. Climatic Change 23:111119.

Penner, J.E., C.S. Atherton, J. Dignon, S.J. Ghan, J.J. Waiton, and S. Hameed (1991) Tropospheric nitrogen: a three-dimensional study of sources, distributions, and deposition. Journal of Geophysical Research 96:959-990.

Penner, J.E., C.S. Atherton, and T.E. Graedel (1994) Global emissions and models of photochemically active compounds. In Global Atmospheric-Biospheric Chemistry (R.G. Prinn, Ed.), Plenum Press, New York, pp.237-247.

Peterson, B.J. and J.M. Melillo (1985) The potential storage of carbon caused by eutrophication of the biosphere. Tellus 37B:117-127.

Pierce, L.L. (1993) Scaling ecosystem models from watersheds to regions: Tradeoffs between model complexity and accuracy. Ph.D. dissertation, School of Forestry, Univ. of Montana.

Pinty, B., and M.M. Verstraete (1992) GEMI: a non-linear index to monitor global vegetation from satellites. Vegetatio 101:15-20.

Post, W.M., J. Pastor, P.J. Zinke, and A.G. Stangenberger (1985) Global patterns of soil nitrogen storage. Nature 317:613-616.

Potter, C.S., J.T. Randerson, C.B. Field, P.A. Matson, P.M. Vitousek, H.A. Mooney, and S.A. Klooster (1993) Terrestrial ecosystem production: A process model based on global satellite and surface data. Global Biogeochemical Cycles 7:811-841.

Prather, M., R. Derwent, D. Ehhalt, P. Fraser, E. Sanhueza, and X. Zhou (1995) Other trace gases and atmospheric chemistry. In Climate Change 1994: Radiative Forcing of Climate Change (J.T. Houghton et al. Eds.), Cambridge University Press, Cambridge. pp. 73-126.

Prince, S.D. and S.N. Goward (1996) Evaluation of the NOAA/NASA Pathfinder AVHRR Land Data Set for global primary production modeling. International Journal of Remote Sensing 17:217-221.

Prinn, R.G. (1994) The interactive atmosphere: Global atmospheric-biospheric chemistry. Ambio 23:50-61.

Privette, J.L., R.B. Myneni. C.J. Tucker, and W.J. Emery (1994) Invertability of a 1-D discrete ordinates canopy reflectance model. Remote Sensing of Environment 48:89-105.

Privette, J.L., W.J. Emery, R.B. Myneni, and D.S. Schimel. (In press) Inversion of a vegetation reflectance model with NOAA AVHRR data. Remote Sensing of Environment.

Qin. W.-H. (1993) Modeling bidirectional reflectance of multicomponent canopies. Remote Sensing of Environment 46:235-245.

Quay, P.D., B. Tilbrook, and C.S. Wong (1992) Oceanic uptake of fossil fuel $\mathrm{CO}_{2}$ : carbon-13 evidence. Science 256:74-79.

Rastetter, E.B., A.W. King, B.J. Cosby, G.M. Hornberger, R.V. O'Neil, and J.E. Hobbie (1992) Aggregating fine-scale ecological knowledge to model coarser-scale attributes of ecosystems. Ecological Applications 2:55-70.

Rayleigh, A. Lord (1914) In Proceedings of the Royal Society A90:219.

Raynaud, D. and J.-M. Barnola (1985) An Antarctic ice core reveals $\mathrm{CO}_{2}$ : The major uncertainties. Global Biogeochemical Cycles 5:309-314.

Reich, P.B. (1987) Quantifying plant response to ozone: A unifying theory. Tree Physiology 3:63-91. 
Roelofs, G.-J. and J. Lelieveld (1995) Distribution and Budget of $\mathrm{O}_{3}$ in the troposphere calculated with a chemistry-general circulation model. Journal of Geophysical Research 100D:20983-20988.

Rosenzweig, M.L. (1968) Net primary productivity of terrestrial communities: Prediction from climatological data. American Naturalist 102:67-74.

Ross, J. (1975) Radiative transfer in plant communities. In Vegetation and the Atmosphere (J.L. Monteith, Ed.), Academic Press, London, pp.13-52.

Ross, I.U. (1981) The Radiation Regime and Architecture of Plant Stands. Dr. W. Junk Publishers, The Hague, Netherlands.

Rotty, R. M., and G. Marland (1986) Production of $\mathrm{CO}_{2}$ from fossil fuel burning by fuel type, 1860-1982. Report NDP-006, CDIAC: Oak Ridge National Laboratory, USA.

Roujean. J.-L., and F.-M. Breon (1995) Estimating PAR absorbed by vegetation from bidirectional reflectance measurements. Remote Sensing of Environment 51:375-384.

Ruimy. A., B. Saugier and G. Dedieu (1994) Methodology for the estimation of terrestrial net primary production from remotely sensed data. Journal of Geophysical Research 99:52635283.

Running, S.W., R.R. Nemani. D.L. Peterson, L.E. Band, D.F. Potts. L.L. Pierce, and M.A. Spanner (1989) Mapping regional forest evapotranspiration and photosynthesis by coupling satellite data with ecosystem simulation. Ecology 70:1090-1 101.

Running, S.W. and R.R. Nemani (1991) Regional hydraulic and carbon balance responses of forests resulting from potential climate change. Climatic Change 19:349-368.

Running, S.W. and E.R. Hunt Jr. (1993) Generalization of a forest ecosystem model for other biomes, BIOME-BGC, and an application for global-scale models. In Scaling Physiological Processes: Leaf to Globe, J.R. Ehleringer and C.B. Field (Eds.), pp. 141-158, Academic Press, San Diego.

Running, S., T.R. Loveland, E.R. Hunt (1995) A remote sensing based vegetation classification logic for global land cover analysis. Remote Sensing of Environment 51:3948.

Ryde, J.W. (1931) In Proceedings of the Royal Society A90:219.

Sala, O.E., W.J. Parton, L.A. Joyce, and W.K. Lauenroth (1988) Primary production of the central grasslands region of the United States: Spatial pattern and major controls. Ecology 69:40-45.

Sarmiento, J. (1991) Oceanic uptake of $\mathrm{CO}_{2}$ : The major uncertainties. Global Biogeochemical Cycles 5:309-314.

Sarmiento, J. and U. Siegenthaler (1992) New production and the global carbon cycle. In Primary Productivity and Biogeochemical Cycles in the Sea (P.G. Falkowski and A.D. Woodhead, Eds.), Plenum Press, New York.

Sarmiento, J. (1993) Atmospheric $\mathrm{CO}_{2}$ stalled. Nature 365:697-698.

Schimel, D.S., M.A. Stillwell, and R.G. Woodmansee (1985) Biogeochemistry of C, N, and P in a soil catena of the shortgrass steppe. Ecology 66:276-282.

Schimel, D.S., W.J. Parton, T.G.F. Kittel, D.S. Ojima, and C.V. Cole (1990) Grassland biogeochemistry: Links to atmospheric processes. Climatic Change 17:13-25.

Schimel, D.S., T.G.F. Kittel, and W.J. Parton (1991) Terrestrial biogeochemical cycles: Global interactions with atmosphere and hydrology. Tellus 43:188-203.

Schimel, D.S., B.H. Braswell, E.A. Holland, R. McKeown, D.S. Ojima, T.H. Painter, W.J. Parton, and A.R. Townsend (1994) Climatic, edaphic and biotic controls over storage and urnover of carbon in soils. Global Biogeochemical Cycles 8:279-293. 
Schimel. D.S. (1995a) Terrestrial biogeochemical cycles: Global estimates with remote sensing. Remote Sensing of Environment 51:49-56.

Schimel, D.S. (1995b) Terrestrial ecosystems and the carbon cycle. Global Change Biology 1:77-91.

Schimel, D., I.G. Enting, M. Heimann, T.M.L. Wigley, D. Raynaud, D. Alves, and U. Siegenthaler, et al. (1995) $\mathrm{CO}_{2}$ and the carbon cycle. In Climate Change 1994: Radiative Forcing of Climate Change and An Evaluation of the IPCC IS92 Emission Scenarios, J.T. Houghton, L.G. Meira Filho, J. Bruce, H. Lee, B.A. Callander, E. Haites, N. Harris and K. Maskell (Eds.), pp. 35-71, Cambridge University Press, Cambridge, UK.

Schimel, D.S., B.H. Braswell, R. McKeown, D.S. Ojima, W.J. Parton, and W. Pulliam (In press a) Climate and nitrogen controls on the geography and time scales of terrestrial biogeochemical cycling. Global Biogeochemical Cycles.

Schimel, D.S., VEMAP Participants, and B.H. Braswell (In press b) Spatial variability in ecosystem processes at the continental scale: models, data, and the role of disturbance. Ecology.

Schindler, D.W., and S.E. Bayley (1993) The biosphere as an increasing sink for atmospheric carbon: estimates from increased nitrogen deposition. Global Biogeochemical Cycles. 7:717-733.

Schlesinger, W. H. (1991) Biogeochemistry: An Analysis of Global Change. Academic Press: San Diego, CA.

Schlesinger, W.H. and A.E. Harley (1992) A global budget for atmospheric $\mathrm{NH}_{3}$. Biogeochemistry 15:191-211.

Schulze, E.-D. (1989) Air pollution and forest decline in a spruce (Picea abies) forest. Science 244:776-783.

Schulze, E.-D., W. De Vries, M. Hauhs, K. Rosén, L. Rasmussen, O.-C. Tann, and J. Nilsson (1989) Critical loads for nitrogen deposition in forest ecosystems. Water, Air and Soil Pollution 48:451-456.

Schuster, A. (1905) Radiation through a foggy atmosphere. The Astrophysical Journal 21:1-22.

Sedjo, R.A. (1992) Temperate forest ecosystems in the global carbon cycle. Ambio 21:274277.

Sellers, P.J., Y. Mintz, Y.C. Sud, and A. Dalcher (1986) A simple biosphere model (SiB) for use within general circulation models. Journal of Atmospheric Science, 43:505-531.

Sellers, P.J. (1987) Canopy reflectance, photosynthesis, and transpiration II: the role of biophysics in the linearity of their interdependence. International Journal of Remote Sensing 6:1335-1372.

Sellers, P. J., J. A. Berry, G. J. Collatz, C. B. Field, and F. G. Hall (1992) Canopy reflectance. photosynthesis, and transpiration. III. A reanalysis using improved leaf models and a new canopy integration scheme. Remote Sensing of Environment 42:187-216.

Sellers, P.J. and D.S. Schimel (1993) Remote sensing of the land biosphere and biogeochemistry in the EOS era: science priorities, methods and implementation - EOS land biosphere and biogeochemical panels. Global and Planetary Change 7:279-297.

Sellers, P.J., C.J. Tucker, G.J. Collatz, S.O. Los, C.O. Justice, D.A. Dazlich, and D.A. Randall (1994) A Global $1^{\circ}$ by $1^{\circ}$ data set for climate studies. Part 2: The generation of global fields of terrestrial biophysical parameters from the NDVI. International Journal of Remote Sensing 15:3519-3545.

Sellers, P.J., L. Bounoua, G.J. Collatz, D.A. Randall, D.A. Dazlich, S.O. Los, J.A. Berry, I. Fung, C.J. Tucker, C.B. Field, and T.G. Jensen (1996) Comparison of radiative and physiological effects of doubled atmospheric $\mathrm{CO}_{2}$ on climate. Science 271:1402-1406.

Selvin, S. (1995) Practical Biostatistical Methods. Duxbury Press, Belmont, Calif., pp.95-135. 
Shaver G.R., W.D. Billings, F.S. Chapin, A.E. Giblin, K.J. Nadelhoffer, W.C. Oeschel, and E.B. Rastetter (1993) Global change and the carbon balance of arctic ecosystems. Bioscience 42:433-441.

Shepherd, M.F., S. Barzetti, and D.R. Hastie (1991) The production of atmospheric $\mathrm{NO}_{\mathrm{x}}$ and $\mathrm{N}_{2} \mathrm{O}$ from an agricultural soil. Atmospheric Environment 25:1961-1969.

Shine, K. P., R. G. Derwent, D. J. Wuebbles, and J-J. Morcrette (1990) Greenhouse gases and aerosols. In Climate Change: The IPCC Scientific Assessment, Cambridge University Press.

Siegenthaler, U. (1983) Uptake of excess $\mathrm{CO}_{2}$ by an outcrop-diffusion model of the ocean. Journal of Geophysical Research 88:3599-3608.

Siegenthaler, $U$. and $\mathrm{H}$. Oeschger (1987) Biospheric $\mathrm{CO}_{2}$ emissions during the past 200 years reconstructed by deconvolution of ice core data. Tellus 39B:140-154.

Siegenthaler, U. (1989) Glacial-interglacial atmospheric $\mathrm{CO}_{2}$ variations. In Global Changes of the Past, UCAR/Office of Interdisciplinary Studies: Boulder, CO.

Siegenthaler, U. and J.L. Sarmiento (1993) Atmospheric carbon dioxide and the ocean. Nature 365:119-125.

Silberstein, L. (1927) In Philosophical Magazine 4:129.

Singh, S.M. and R.J. Saull (1988) The effect of atmospheric correction on the interpretation of multitemporal AVHRR-derived vegetation index dynamics. Remote Sensing of Environment 25:37-51.

Skole, D. and C. Tucker (1993) Tropical deforestation and habitat fragmentation in the Amazon: Satellite data from 1978 to 1988. Science 260:1905-1910.

Spath, H. (1980) Cluster Analysis Algorithms. Ellis Horwood Limited, West Sussex, England.

Spencer, R.W., J.R. Christy, N.C. Grody (1990) Global atmospheric temperature monitoring with satellite microwave measurements: Methods and results 1979-84. Journal of Climate 3:1111-1128.

Spencer, R.W. and J.R. Christy (1992) Precision and radiosonde validation of satellite gridpoint temperature anomalies. Part I: MSU channel 2. Journal of Climate 5:847-857.

Sprent, J.I. (1987) The Ecology of the Nitrogen Cycle. Cambridge University Press, Cambridge.

Stoddard, J.L. (1994) Long-term changes in watershed retention of nitrogen: Its causes and aquatic consequences. In Environmental Chemistry of Lakes and Reservoirs, L.A. Baker, (Ed.), American Chemical Society, pp. 223-284.

Strain, B. R. and J. D. Cure (1985) Direct effects of increasing carbon dioxide on vegetation. United States Department of Energy, NTIS, Springfield, Virginia, USA.

Suits, G. (1972) The calculation of the directional reflectance of a vegetative canopy. Remote Sensing of Environment 2, 117-125.

Tans, P.P., T.J. Conway, and T. Nakazawa (1989) Latitudinal distribution of the sources and sinks of atmospheric carbon dioxide derived from surface observations and an atmospheric transport model. Journal of Geophysical Research 94:5151-5172.

Tans, P.P., I.Y. Fung, T. Takahashi (1990) Observational constraints on the global $\mathrm{CO}_{2}$ budget. Science 247:1431-1438.

Thompson, M.V., J.T. Randerson, C.B. Field, and C.M. Malmstrom, Constraining terrestrial net primary production from estimates of historical plant carbon uptake, paper presented at the IGBP GAIM First Scientific Conference Proceedings, Garmisch-Partenkirchen, Germany, 24-29 Sept., 1995.

Townsend, A.R., P.M. Vitousek, and S.E. Trumbore (1995) Soil organic matter dynamics along gradients in temperature and land use on the island of Hawaii. Ecology 76:721-733. 
Townsend, A.R., B.H. Braswell, E.A. Holland, and J.E. Penner (1996) Spatial and temporal patterns in potential terrestrial carbon storage resulting from deposition of fossil fuel derived nitrogen. Ecological Applications 6:806-814.

Townshend, J.G.R., C.O. Justice, D. Skole, J.-P. Malingreau, J. Cihlar, J. Teillet, F. Sadowski, and S. Ruttenberg. (1994) The 1-km AVHRR global data set: needs of the International Geosphere Biosphere Program. International Journal of Remote Sensing, 15:3319-3332.

Trumbore, S.E. (1993) Comparison of carbon dynamics in tropical and temperate soils using radiocarbon measurements. Global Biogeochemical Cycles 7:273-290.

Tucker, C.J. (1979) Red and photographic linear combinations for monitoring vegetation. Remote Sensing of Environment 8:127-150.

Tucker, C.J. and P.J. Sellers (1986) Satellite remote sensing of primary production. International Journal of Remote Sensing 7:1395-1416.

Uchijima, Z. and H. Seino (1985) Agroclimatic evaluation of net primary productivity of natural vegetations. Journal of Agricultural Meteorology 40:343-352.

VEMAP (1995) Vegetation/Ecosystem Modeling and Analysis Project: Comparing biogeography and biogeochemistry models in a continental-scale study of terrestrial ecosystem responses to climate change and $\mathrm{CO}_{2}$ doubling. Global Biogeochemical Cycles 9:407-437.

Verhoef, W. (1984) Light scattering by leaf layers with application to canopy reflectance modeling: the SAIL model. Remore Sensing of Environment 16:125-141.

Verhoef, W. (1985) Earth observation modeling based on layer scattering matrices. Remote Sensing of Environment 17:165-178.

Vitousek, P.M. and R. L. Sanford (1986) Nutrient cycling in moist tropical forests. Annual Review of Ecology and Systematics 17:137-167.

Vitousek, P.M., T. Fahey, D.W. Johnson, and M.J. Swift (1988) Element interactions in forest ecosystems: succession, allometry and input-output budgets. Biogeochemistry 5:7-34.

Vitousek, P.M. and R.W. Howarth (1991) Nitrogen limitation on land and in the sea: how can it occur? Biogeochemistry 13:87-115.

Walthall, C.J., J.M. Norman, J.M. Welles, G. Campbell, B.L. Blad (1985) Simple equation to approximate the bidirectional reflectance from vegetated canopies and bare soil surfaces. Applied Optics 24:383-387.

Watson, R.T., H. Rodhe, H. Oeschger, and U. Siegenthaler (1990) Greenhouse gases and aerosols. In Climate Change: The IPCC Scientific Assessment, Cambridge University Press. pp. $1-40$.

Watson, R.T., L.G. Meira Filho, E. Sanhueza, A. Janetos (1992) Greenhouse gases: sources and sinks. In, Climate Change 1992: The Supplementary Report to the IPCC Scientific Assessment, Cambridge University Press, pp. 25-46.

Webber, A.N., G.-Y. Nie, and S.P. Long (1994) Acclimation of photosynthetic proteins to rising atmospheric $\mathrm{CO}_{2}$. Photosynthesis Research 39:413-425.

Wessman, C.A., J.D. Aber, and J.M. Melillo (1988) Remote sensing of canopy chemistry and nitrogen cycling in temperate forest ecosystems. Nature 335:154-156.

White, J.W.C., P. Ciais, R.A. Figge, R. Kinney, and V. Markgraf (1994) A high resolution record of atmospheric $\mathrm{CO} 2$ content from carbon isotopes in peat. Science 367:153-156.

Wiegand, C.L., A.J. Richardson, D.E. Escobar, and A.H. Gerbermann (1991) Vegetation indices in crop assessments. Remote Sensing of Environment 35:105-119.

Wofsy, S.C., M.L. Goulden, J.W. Munger, S.-M. Fan, P.S. Bakwin, B.C. Daube, S.L. Bassow, and F.A. Bazzaz (1993) Net exchange of $\mathrm{CO}_{2}$ in a mid-latitude forest. Science 260:1314-1317. 
Woodwell, G.M., R.H. Whittaker, W.A. Reiners, G.E. Likens, C.C. Delwiche, and D.B. Botkin, Science 199: 141

Wyatt, C.L. (1978) Radiometric Calibration: Theory and Methods. Academic Press, New York.

Yienger, J.J. and H. Levy II (1995) An empirical model of global soil-biogenic $\mathrm{NO}_{\mathrm{x}}$ emissions. Journal of Geophysical Research 100D:11447-11464.

Zak, D.R., D. Tilman, R.R. Parmenter, C.W. Rice, F.M. Fisher, J. Vose, D. Milchunas, and C.W. Martin (1994) Plant production and soil microorganisms in late-successional ecosystems: A continental-scale study. Ecology 75:2333-2347.

Zimmerman, P.H., J. Feichter, H.K. Rath, P.J. Crutzen, and W. Weiss (1989) A global threedimensional source-receptor model investigation using ${ }^{85} \mathrm{Kr}$. Atmospheric Environment 23:25-35.

Zobler, L. (1986) A world soil file for global climate modeling, NASA Tech. Mem. 87802.

Zöttl, H.W. (1990) Remarks on the effects of nitrogen deposition to forest ecosystems. Plant and Soil 128:83-89. 